\title{
Study of the Variations in Continuous Diesel Particulate Matter Size Measurements and Effect of Fuel Properties on DPM Size
}

Kevin Anthony Chappell Flaim

West Virginia University

Follow this and additional works at: https://researchrepository.wvu.edu/etd

\section{Recommended Citation}

Flaim, Kevin Anthony Chappell, "Study of the Variations in Continuous Diesel Particulate Matter Size Measurements and Effect of Fuel Properties on DPM Size" (2008). Graduate Theses, Dissertations, and Problem Reports. 4373.

https://researchrepository.wvu.edu/etd/4373

This Thesis is protected by copyright and/or related rights. It has been brought to you by the The Research Repository @ WVU with permission from the rights-holder(s). You are free to use this Thesis in any way that is permitted by the copyright and related rights legislation that applies to your use. For other uses you must obtain permission from the rights-holder(s) directly, unless additional rights are indicated by a Creative Commons license in the record and/ or on the work itself. This Thesis has been accepted for inclusion in WVU Graduate Theses, Dissertations, and Problem Reports collection by an authorized administrator of The Research Repository @ WVU. For more information, please contact researchrepository@mail.wvu.edu. 
Study of the Variations in Continuous Diesel Particulate Matter Size Measurements and Effect of Fuel Properties on DPM Size

\author{
Kevin Anthony Chappell Flaim
}

Thesis submitted to the

College of Engineering and Mineral Resources

at West Virginia University

in partial fulfillment of the requirements

for the degree of

\author{
Master of Science \\ in \\ Mechanical Engineering
Gregory J. Thompson, Ph.D., Chair
Nigel N. Clark, Ph.D.
Mridul Gautam, Ph.D. \\ Department of Mechanical Engineering
}

Morgantown, West Virginia
2008

Keywords: Diesel Particulate Matter, Particle Sizing, Additives, FTP

Copyright 2008 Kevin Flaim 


\title{
ABSTRACT \\ Study of the Variations in Continuous Diesel Particulate Matter Size Measurements and Effect of Fuel Properties on DPM Size
}

\author{
Kevin Anthony Chappell Flaim
}

Heavy-duty diesel engines (HDDE), because of their widespread use and reputation of expelling excessive soot, have frequently been held responsible for superfluous amounts of overall environmental particulate matter (PM). PM is a considerable contributor to air pollution, and a subject of primary concern to health and regulatory agencies worldwide. The U.S. Environmental Protection Agency (EPA) has provided PM emissions regulations and standards of measurement techniques since the 1980's. PM standards set forth by the EPA for HDDEs are based only on total mass, instead of size and/or concentration. The European Union is considering regulating particle size, and it may influence the U.S. EPA to adopt particle size limits in the future.

The purpose of this research was to better understand the variations in DPM measurements due to fuel alteration or changes in other parameters such as relative humidity and dilution ratio. The Cambustion DMS-500, a fast particle spectrometer, was used to continuously sample diesel aerosol from a HDDE test-cell dilution tunnel. Samples were taken from the primary dilution tunnel and were further diluted in a secondary dilution system. Various fuels were selected to represent available market supplies. Three engines, a 2004 Cummins ISM 370, a 1992 Detroit Diesel DDC S60, and a rebuilt 1992 Detroit Diesel DDC S60, were tested to examine the effects of varying engine technology. Several types of biodiesel and petroleum-based diesel fuels were evaluated in these engines with and without additives. Experiments were performed to determine: the minimum detectable variations in PM concentrations, relative humidity effects, dilution effects, barometric pressure effects, fuel additive effects, sample line temperature effects, and the associations between certain engine operating events and PM concentrations.

The smallest significant variation in concentration that was detected during this research was a $1.51 \%$ increase in $64 \mathrm{~nm}$ particles due to the use of a fuel additive. The effects of relative humidity on particle concentrations were only present during low load (less than $50 \%$ of the maximum load) engine operation, and they were generally associated with high levels of run-to-run variation. Nearly doubling the overall dilution ratio was shown to greatly increase nanoparticle $(<40 \mathrm{~nm})$ concentrations and slightly increase the concentrations of other particles during certain operation conditions. Barometric pressure change was not directly linked to PM emissions, but it appeared to have caused increased run-to-run variation. In all cases where a fuel additive was used, an increase in the amount of the additive led to a $130 \%$ to $-90 \%$ change in the resulting

particle concentrations. In addition, a technique was developed for the conversion of continuous DMS data (particle sizes and concentrations) to brake-specific PM mass-flow. According to the gravimetric measurements, the technique was accurate to within $6.24 \%$ for the 1992 DDC S60 engines and $26.4 \%$ for the 2004 ISM 370 engine. 


\section{ACKNOWLEDGEMENTS}

I believe that the challenge of completing this research has prepared me sufficiently for the obstacles to come in my professional career. I will be content with my work so long as a single reader finds significance in it. My goals as a graduate student were achieved only because of the support that flowed from the people around me. Therefore, I have many thanks to give.

I would like to thank:

Lauren Lambert, John Nuszkowski, Mike Ursic, and the members of my committee for helping me with the revisions of my thesis,

my advisor, Gregory Thompson, for consistently being patient, helpful, and a reliable source of guidance,

the faculty members and employees at the ERC who took time to lend a helping hand, Bradley Ralston for teaching me about the test cell and assisting in several projects, my parents for giving me every chance to succeed; and specifically, my mother, Susan Chappell, for always listening, keeping me on track, and being positive and encouraging,

my father, Dr. Anthony Flaim, for understanding when I said "I just need to get some work done this weekend,"

my brother, Nathan Flaim, for providing motivation by giving me terrific dreams about our futures, each of my four grandparents for having that gleam in their eyes that says they're proud, my uncle, Gino Flaim, for coming up with inspiringly clever ideas that make me feel like my career choice is fun and exciting, and my high school English teacher, Benny Mills, for teaching me practically everything I know about writing.

I would also like to thank God for the way things often unfold. Please pardon the lengthiness of this thesis. There was much to explain. Besides, you can't stand on a thin book to reach a high shelf. 


\section{TABLE OF CONTENTS}

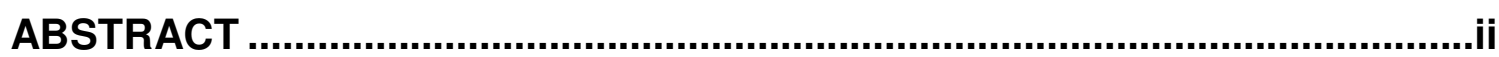

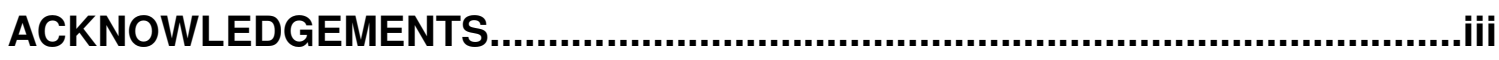

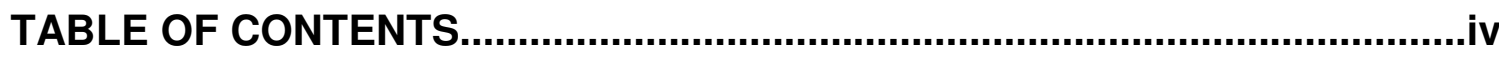

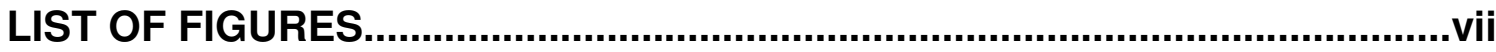

LIST OF TABLES ............................................................................................

LIST OF ACRONYMS AND ABBREVIATIONS...........................................,XV

LIST OF SYMBOLS .......................................................................................

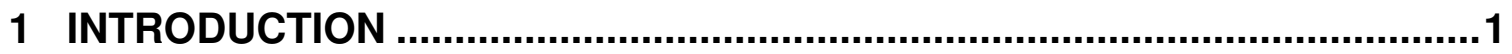

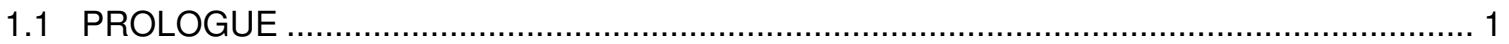

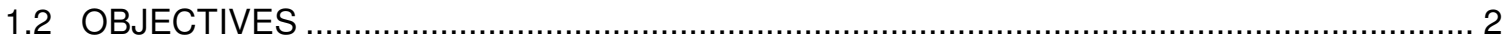

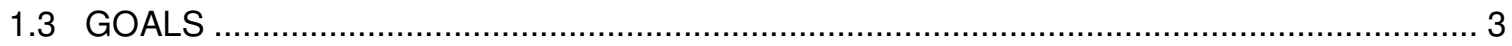

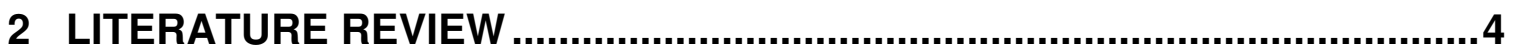

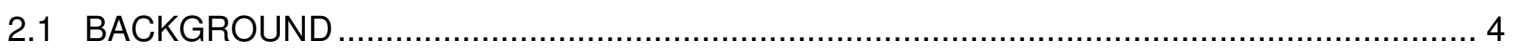

2.2 EPA STANDARDS AND REGULATIONS

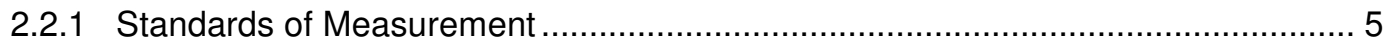

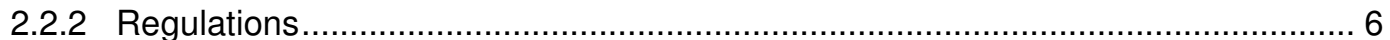

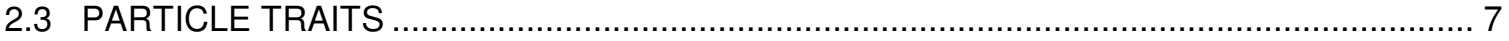

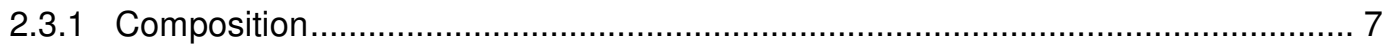

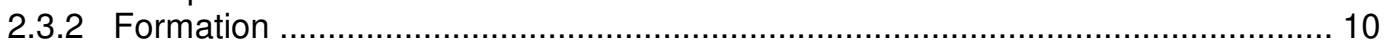

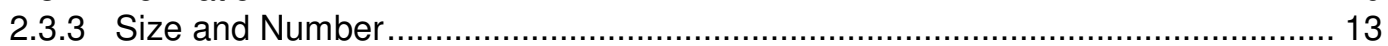

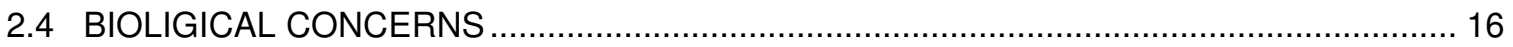

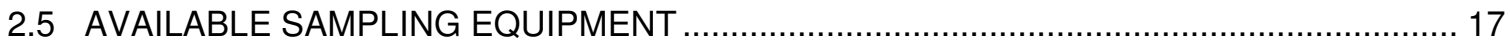

2.5.1 Nanometer Aerosol Size Analyzer (n-ASA) .............................................. 17

2.5.2 Differential Mobility Analyzer (DMA) .............................................................. 18

2.5.3 Differential Mobility Particle Spectrometer (DMPS) ........................................ 18

2.5.4 Transmission Electron Microscope (TEM) .................................................... 19

2.5.5 Tapered Element Oscillating Microbalance (TEOM) ....................................... 19

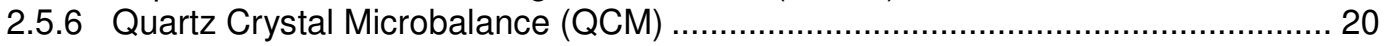

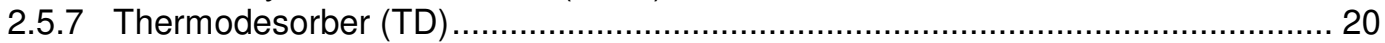

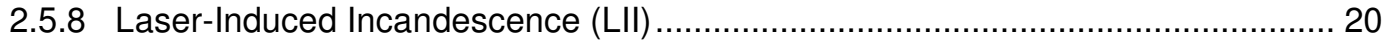

2.5.9 Photoelectric Aerosol Sensor (PAS) ……................................................. 21

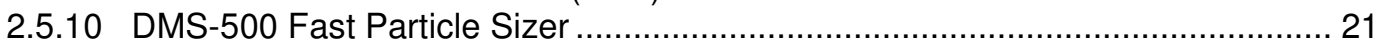

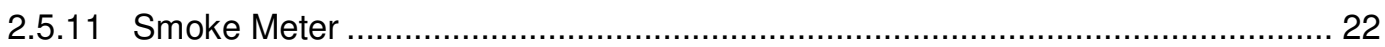

2.5.12 Faraday Cup Electrometer (FCE) ......................................................... 22

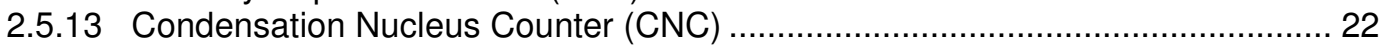

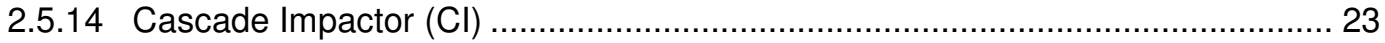

2.5.15 Parallel Flow Diffusion Battery (PFDB) ….................................................. 23

2.5.16 Electrical Low Pressure Impactor (ELPI)..................................................... 24

2.5.17 Scanning Mobility Particle Sizer (SMPS) ................................................. 25

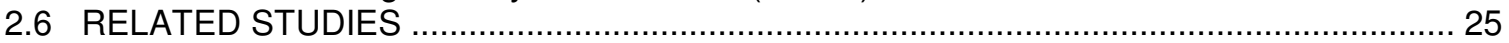

2.6.1 Particle Size Distribution from a Heavy-Duty Diesel Engine: Steady-State and

Transient Emission Measurement Using Two Dilution Systems and Two Fuels ............. 25

2.6.2 Evaluation of Methods for Determining Continuous Particulate Matter from

Transient Testing of Heavy-Duty Diesel Engines........................................................ 26

2.6.3 A Fast Response Particulate Spectrometer for Combustion Aerosols .................. 28

2.6.4 Effective Density of Diesel Exhaust Particles as a Function of Size....................... 29 
2.6.5 Evaluation of Methods for the Determination of Diesel-Generated Fine Particulate

Matter: Physical Characterization Results................................................................... 30

2.6.6 Research on Diesel Exhaust and Other Particulates .......................................... 30

2.6.7 Particles from Internal Combustion Engines - What We Need to Know ................ 31

2.6.8 Evaluation and Correction of Moisture Adsorption and Desorption from a Tapered

Element Oscillating Microbalance 32

2.6.9 Significance of Fuel Sulfur Content and Dilution Conditions on Particle Emissions

from a Heavily-Used Diesel Engine During Transient Operation .................................... 34

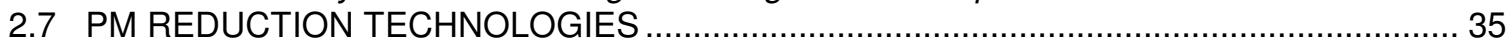

\section{EXPERIMENTAL SETUP AND PROCEDURES .............................................}

3.1 OVERTURE

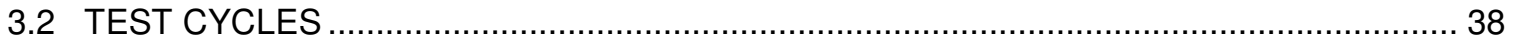

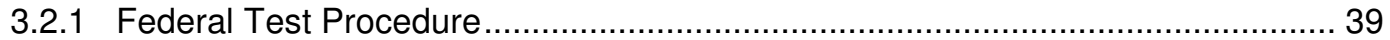

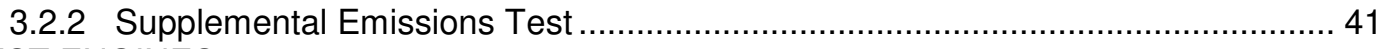

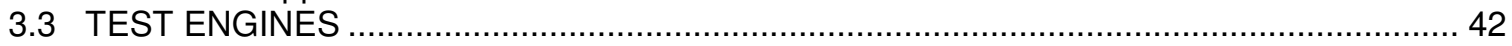

3.3.1 1992 Detroit Diesel Series 60 ..................................................................... 43

3.3.2 Rebuilt 1992 Detroit Diesel Series 60 ........................................................ 44

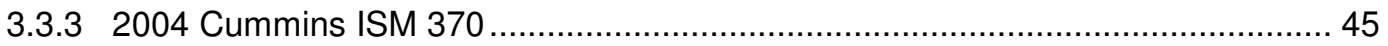

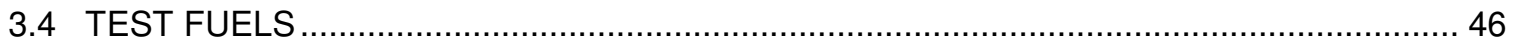

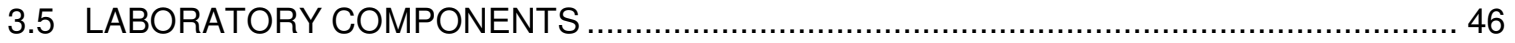

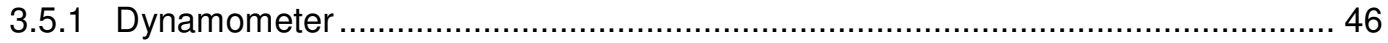

3.5.2 Engine Control and Data Acquisition System.................................................. 47

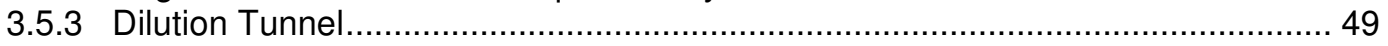

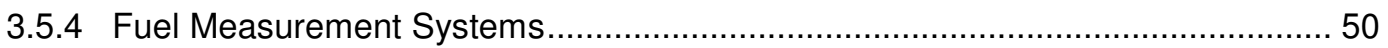

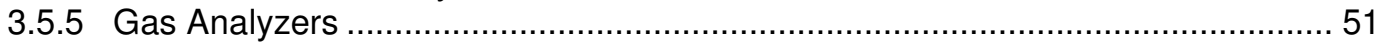

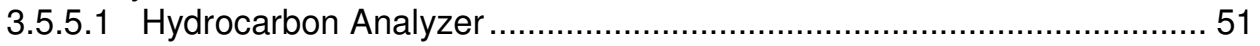

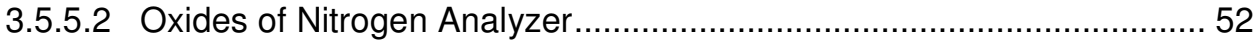

3.5.5.3 Carbon Monoxide Analyzer ………............................................... 52

3.5.5.4 Carbon Dioxide Analyzer................................................................. 52

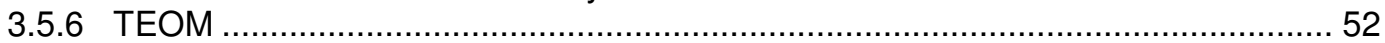

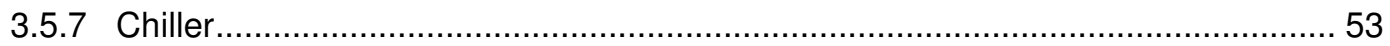

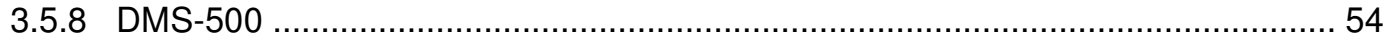

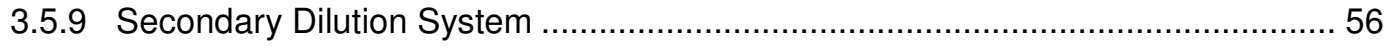

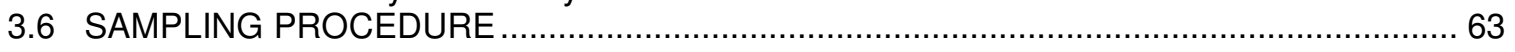

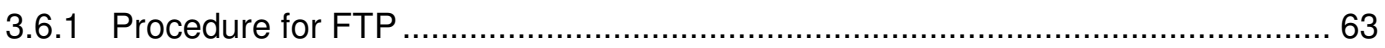

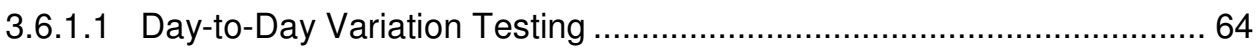

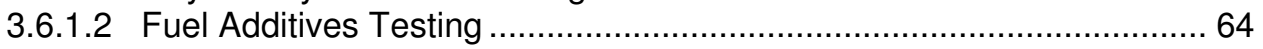

3.6.1.3 Unheated Sample Line Testing ...................................................... 65

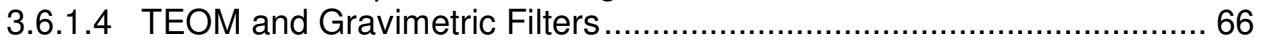

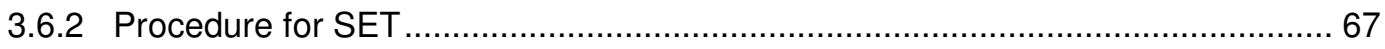

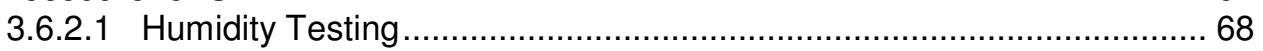

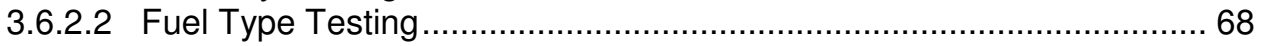

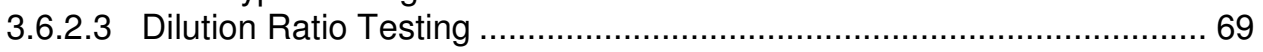

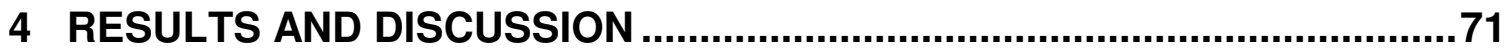

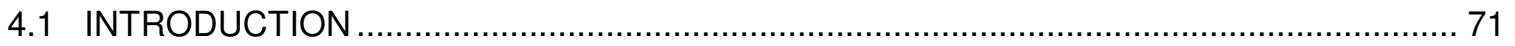

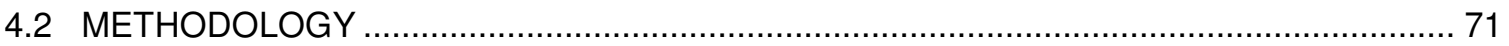

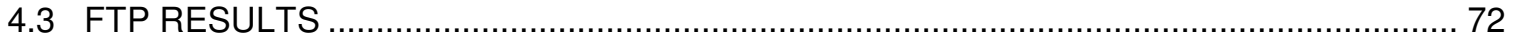

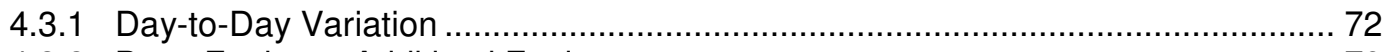

4.3.2 Base Fuels vs. Additized Fuels ................................................................. 79

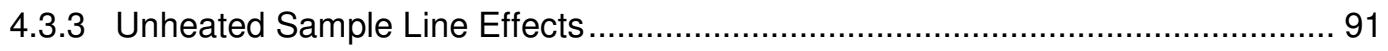

4.3.4 Extremes of Run-to-Run Variation ................................................................ 95

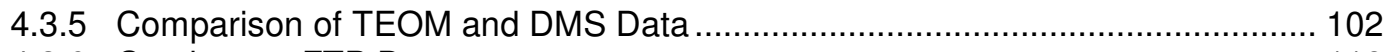

4.3.6 Continuous FTP Data .............................................................................. 118 


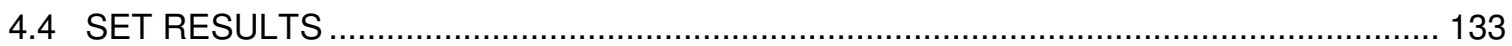

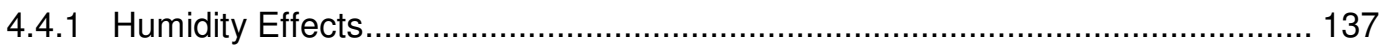

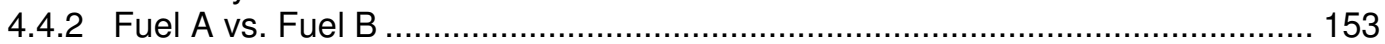

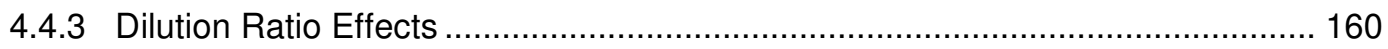

4.4.4 Secondary Dilution Ratio Validation ............................................................. 169

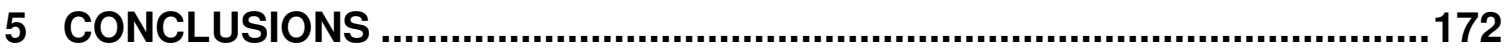

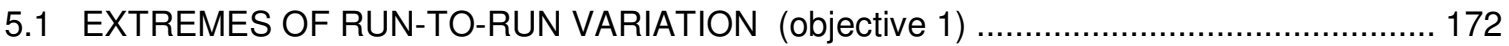

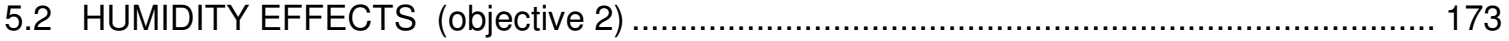

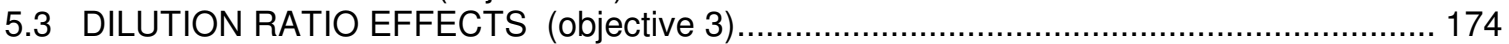

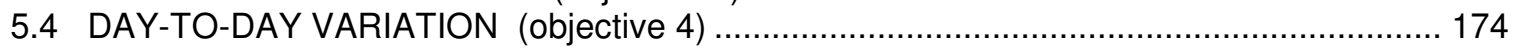

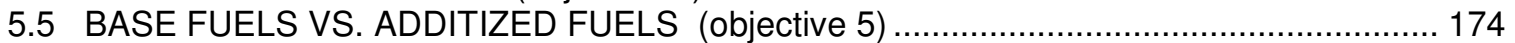

5.6 COMPARISON OF TEOM AND DMS DATA (objective 6) …....................................... 175

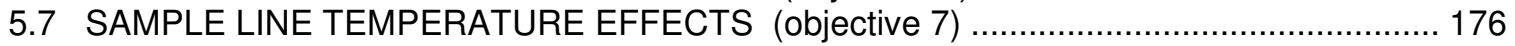

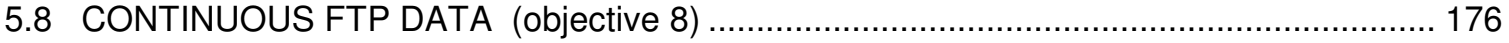

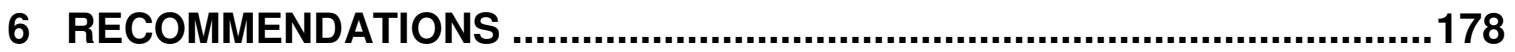

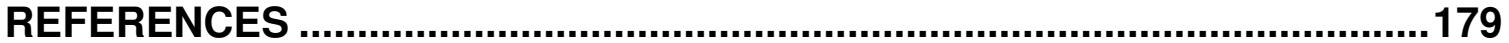

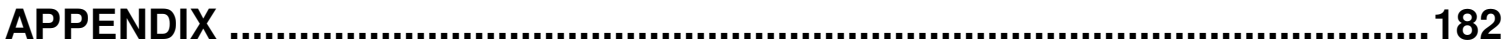

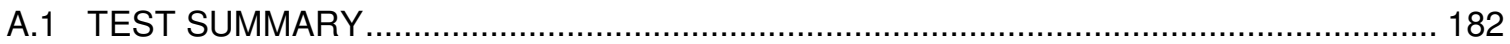

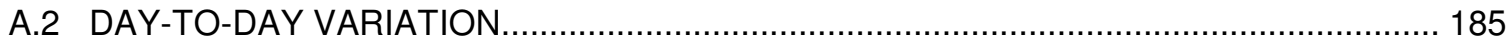

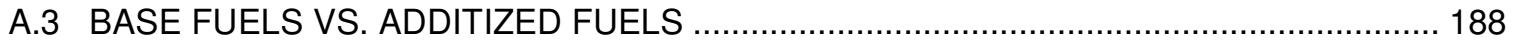

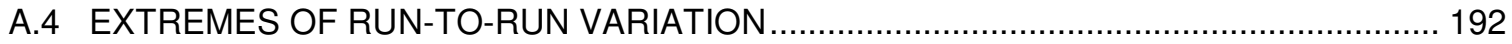

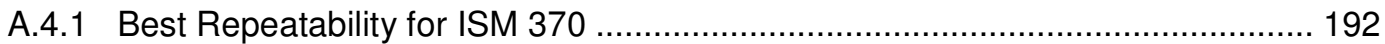

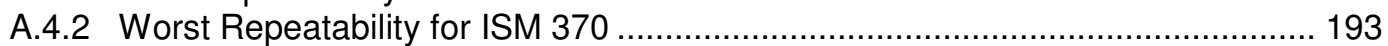

A.4.3 Best Repeatability for DDC S60 .............................................................. 194

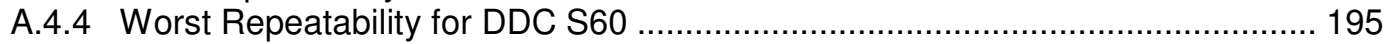

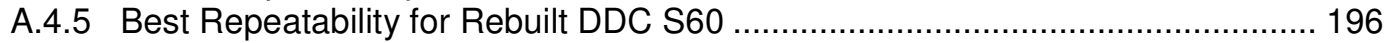

A.4.6 Worst Repeatability for Rebuilt DDC S60 …................................................. 197

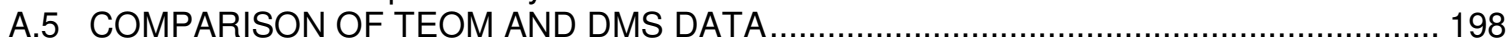

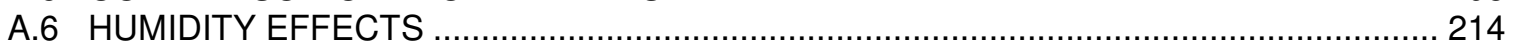

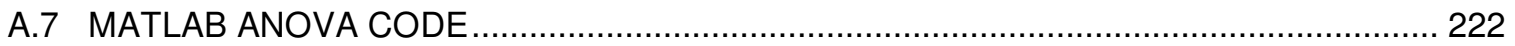

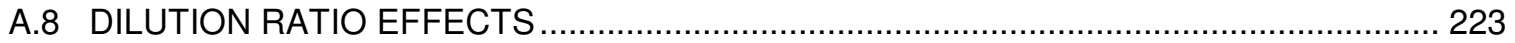

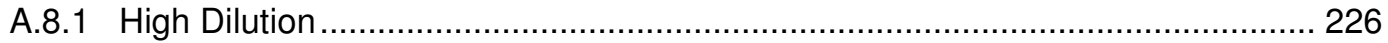

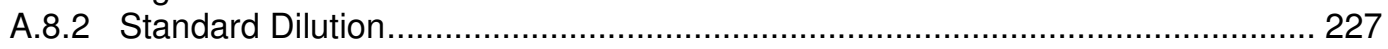

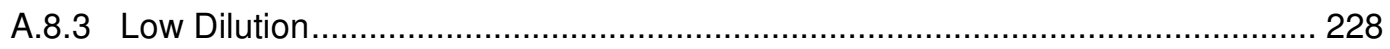




\section{LIST OF FIGURES}

Figure 1 Composition of Diesel Particulate Matter.................................................................... 7

Figure 2 Basic Composition of Nuclei Mode and Accumulation Mode Particles........................... 8

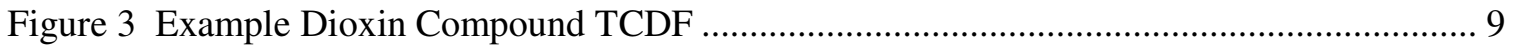

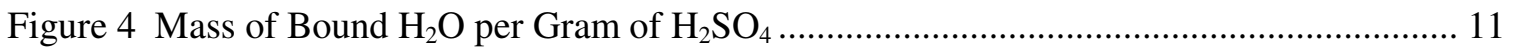

Figure 5 Structure of a Primary Elemental Carbon Particle ....................................................... 12

Figure 6 TEM Picture of a Primary Carbon Particle Attached to an Agglomerated Carbon

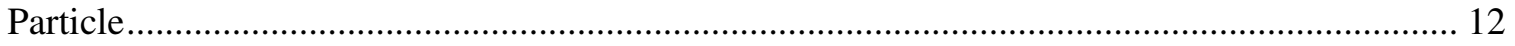

Figure 7 SOF Fractions at Different Operating Conditions....................................................... 13

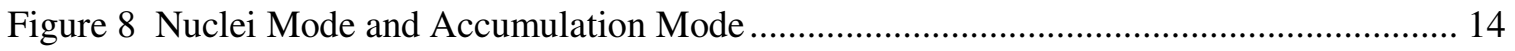

Figure 9 Particulate Number Emissions from Diesel and Gasoline Vehicles ............................. 15

Figure 10 Effect of Residence Time on Particle Number and Size ........................................... 15

Figure 11 Components of Exhaust Sampling System .............................................................. 38

Figure 12 Engine Speed vs. Time for a FTP Cycle ................................................................... 40

Figure 13 Engine Torque vs. Time for a FTP Cycle ............................................................. 40

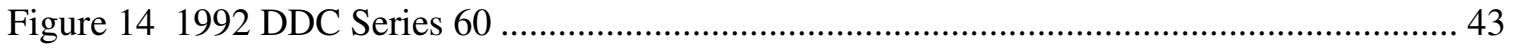

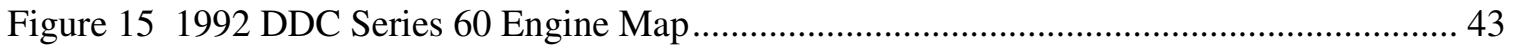

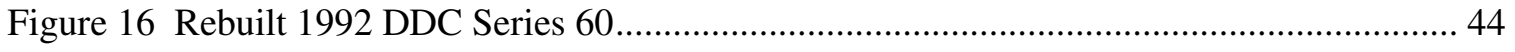

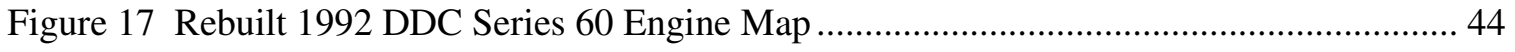

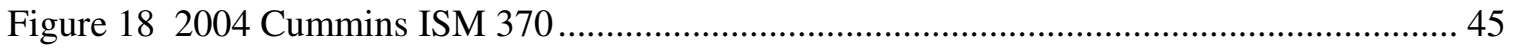

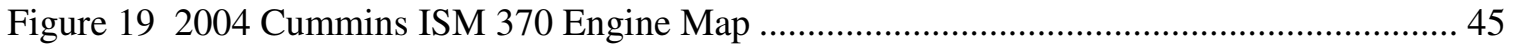

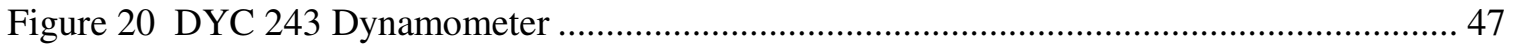

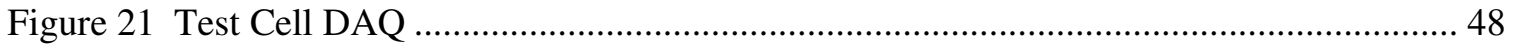

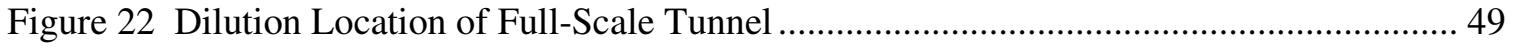

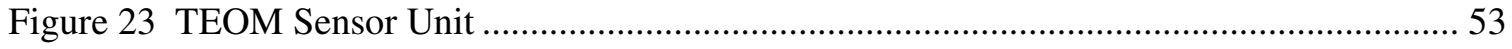

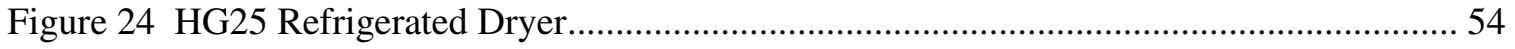

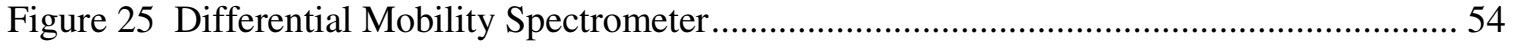

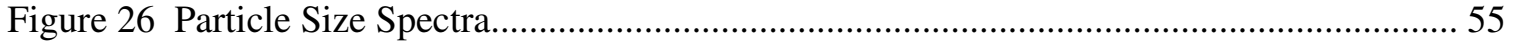

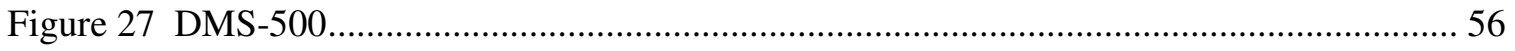

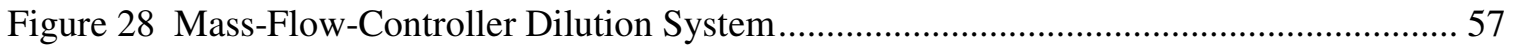

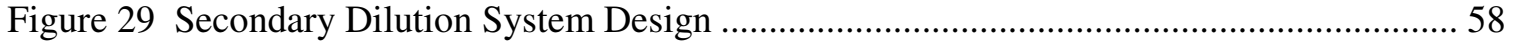

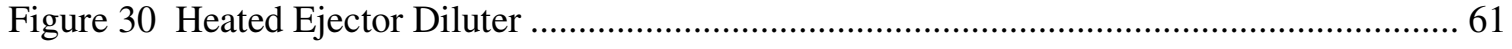

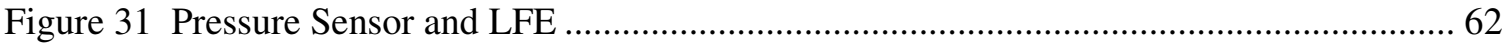

Figure 32 Run-to-Run Variation of the E01927 Test Sequence.................................................. 73 


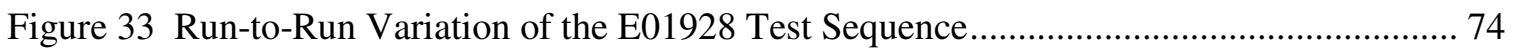

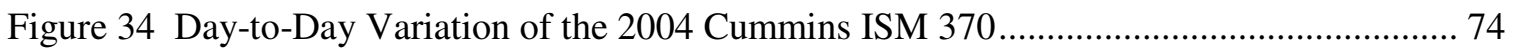

Figure 35 Particle Concentration Comparison of E01927 and E01928 Test Sequences.............. 75

Figure 36 Particle Concentration Differences between E01927 and E01928 Test Sequences..... 76

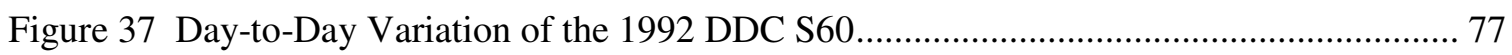

Figure 38 Particle Concentration Differences between E02099 and E02107 Test Sequences..... 78

Figure 39 Particle Concentration Comparison of E02136 and E02138 Test Sequences.............. 80

Figure 40 Particle Concentration Comparison of E02136 and E02140 Test Sequences.............. 81

Figure 41 Differences in Particle Concentrations Due to Mixing of Biodiesel with Low Cetane

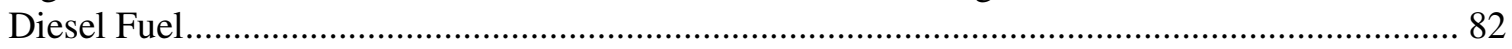

Figure 42 Particle Concentration Comparison of E02195 and E02198 Test Sequences.............. 83

Figure 43 Particle Concentration Comparison of E02195 and E02199 Test Sequences.............. 84

Figure 44 Differences in Particle Concentrations Due to Mixing of Fuel Additives with FEM

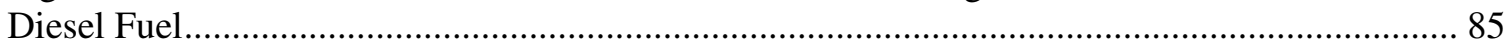

Figure 45 Percent Differences in Particle Distributions Due to Mixing of Additives with Low

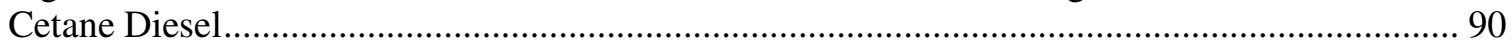

Figure 46 Run-to-Run Variation Using an Unheated Sample Line............................................ 92

Figure 47 Run-to-Run Variation Using a Heated Sample Line................................................. 92

Figure 48 Particle Concentration Comparison of E01967 and E01968 Test Sequences.............. 93

Figure 49 Particle Concentration Differences between Unheated and Heated Sample Line Results 94

Figure 50 Run-to-Run Variations of Test Sequences E01928 and E01921 …........................... 96

Figure 51 Run-to-Run Variations of Test Sequences E01977 and E02099 ................................ 97

Figure 52 Run-to-Run Variations of Test Sequences E02138 and E01959 ................................. 98

Figure 53 Average Particle Concentrations of Test Sequence E02138 ....................................... 99

Figure 54 Average Particle Concentrations of Test Sequence E01921 ..................................... 100

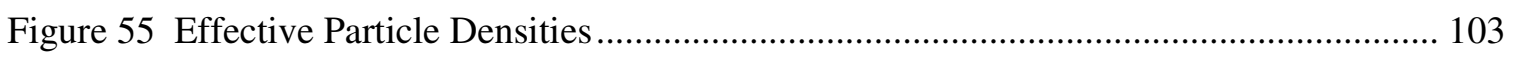

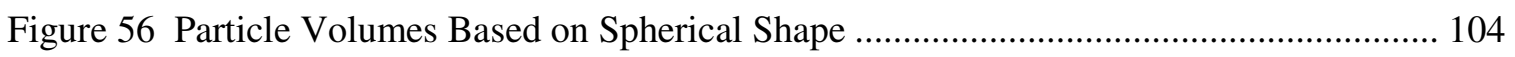

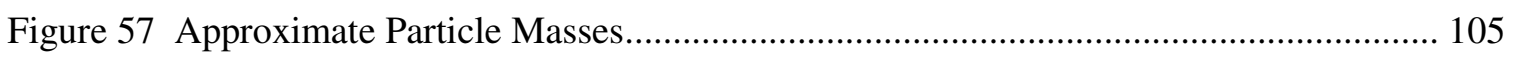

Figure 58 Continuous Particle Distribution of E02123_02 Measured with the DMS-500 ........ 107

Figure 59 PM Mass-Rate of E02123_02 as Measured with the TEOM.................................... 107

Figure 60 Corrected and Uncorrected PM Mass Accumulation of E02123_02 as Measured with

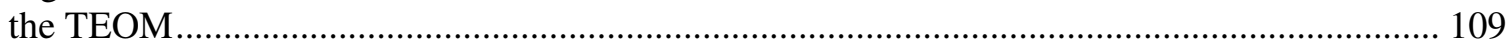

Figure 61 PM Mass-Rates of E02123_02 as Measured with the TEOM and the DMS ............. 112

Figure 62 PM Mass Accumulation of E02123_02 Measured with the TEOM and the DMS .... 114

Figure 63 Run-to-Run Variation of the E01928 Test Sequence ............................................... 118 
Figure 64 Averaged Engine Speed and Torque Curves of E01920........................................ 119

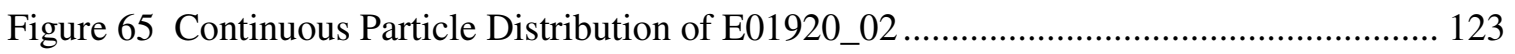

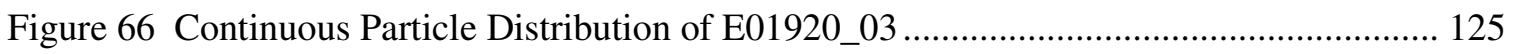

Figure 67 Continuous Particle Distribution of E01920_04 ...................................................... 127

Figure 68 Averaged Overall Dilution Ratio Curve of E01920 ............................................... 131

Figure 69 Engine Speeds and Torques of E01948_01 ............................................................ 134

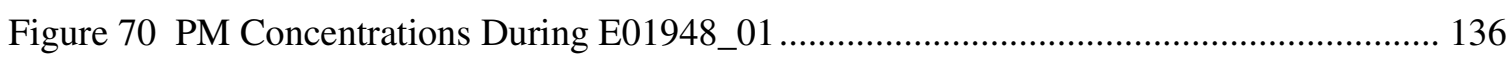

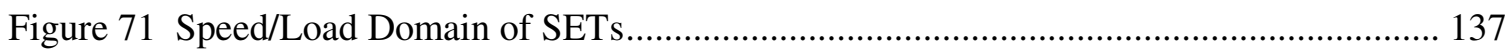

Figure 72 Run-to-Run Variation for Mode 2 of the E02204, E02205, and E02206 Test Sequences 138

Figure 73 Run-to-Run Variation for Mode 5 of the E02204, E02205, and E02206 Test Sequences 139

Figure 74 Run-to-Run Variation for Mode 6 of the E02204, E02205, and E02206 Test Sequences 139

Figure 75 Run-to-Run Variation for Mode 7 of the E02204, E02205, and E02206 Test Sequences 140

Figure 76 Run-to-Run Variation for Mode 10 of the E02204, E02205, and E02206 Test Sequences.......

Figure 77 Run-to-Run Variation for Mode 11 of the E02204, E02205, and E02206 Test

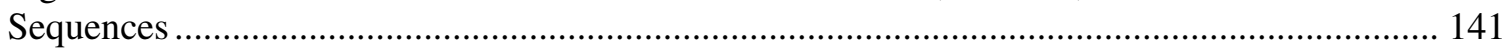

Figure 78 Differences in Particle Concentrations of Mode 2 Due to Humidity Change............ 144

Figure 79 Differences in Particle Concentrations of Mode 5 Due to Humidity Change............ 145

Figure 80 Differences in Particle Concentrations of Mode 6 Due to Humidity Change............ 146

Figure 81 Differences in Particle Concentrations of Mode 7 Due to Humidity Change............ 147

Figure 82 Differences in Particle Concentrations of Mode 10 Due to Humidity Change.......... 148

Figure 83 Differences in Particle Concentrations of Mode 11 Due to Humidity Change.......... 149

Figure 84 Particle Sizes that Have Significantly Different Average Values for Mode 2 Due to

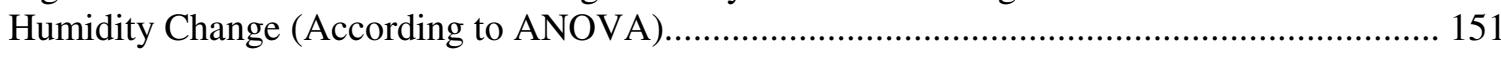

Figure 85 Particle Sizes that Have Significantly Different Average Values for Mode 13 Due to

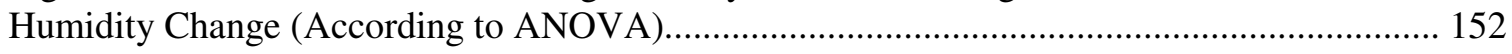

Figure 86 Apparent Fuel Effects of Biodiesel Addition to Low Cetane Diesel for Mode 2 ..... 155

Figure 87 Apparent Fuel Effects of Biodiesel Addition to Low Cetane Diesel for Mode 5 ..... 156

Figure 88 Apparent Fuel Effects of Biodiesel Addition to Low Cetane Diesel for Mode 6 ..... 156

Figure 89 Apparent Fuel Effects of Biodiesel Addition to Low Cetane Diesel for Mode 7 ..... 157

Figure 90 Apparent Fuel Effects of Biodiesel Addition to Low Cetane Diesel for Mode 10 ... 158

Figure 91 Apparent Fuel Effects of Biodiesel Addition to Low Cetane Diesel for Mode 11 ... 159

Figure 92 Apparent Dilution Ratio Effects on Particle Concentrations of Mode 2 2................... 162 
Figure 93 Apparent Dilution Ratio Effects on Particle Concentrations of Mode 5.................... 163

Figure 94 Apparent Dilution Ratio Effects on Particle Concentrations of Mode 6.................... 164

Figure 95 Apparent Dilution Ratio Effects on Particle Concentrations of Mode 7 .................... 165

Figure 96 Apparent Dilution Ratio Effects on Particle Concentrations of Mode 10.................. 166

Figure 97 Apparent Dilution Ratio Effects on Particle Concentrations of Mode 11.................. 167

Figure 98 Calculated Secondary Dilution Ratios of Steady State Test E02206_02 ................... 169

Figure $99 \mathrm{NO}_{\mathrm{x}}$ and Remote $\mathrm{NO}_{\mathrm{x}}$ Concentrations of Steady State Test E02206_02 .................. 170

Figure 100 Average Secondary Dilution Ratios of Humidity-Specific SETs ........................... 171

Figure A-1 Particle Concentration Comparison of E01926 and E01929 Test Sequences.......... 185

Figure A-2 Particle Concentration Comparison of E01972 and E01977 Test Sequences.......... 185

Figure A-3 Particle Concentration Comparison of E02097 and E02107 Test Sequences.......... 186

Figure A-4 Particle Concentration Comparison of E02099 and E02107 Test Sequences.......... 186

Figure A-5 Particle Concentration Comparison of E02101 and E02103 Test Sequences.......... 187

Figure A-6 Particle Concentration Comparison of E02123 and E02124 Test Sequences.......... 188

Figure A-7 Particle Concentration Differences between E02123 and E02124 Test Sequences 188

Figure A-8 Particle Concentration Comparison of E02125 and E02126 Test Sequences.......... 189

Figure A-9 Particle Concentration Comparison of E02125 and E02127 Test Sequences.......... 189

Figure A-10 Particle Concentration Differences between E02125, E02126, and E02127 Test

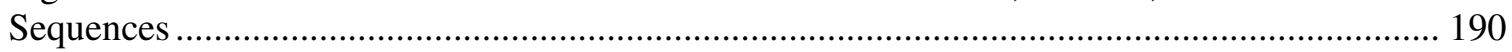

Figure A-11 Particle Concentration Comparison of E01920 and E01926 Test Sequences........ 190

Figure A-12 Particle Concentration Comparison of E01920 and E01928 Test Sequences........ 191

Figure A-13 Particle Concentration Differences between E01920, E01926, and E01928 Test

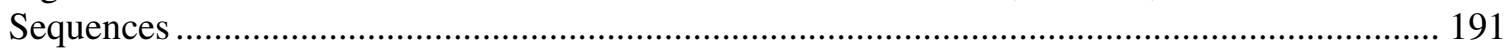

Figure A-14 Average Particle Concentrations of Test Sequence E01928 .................................. 192

Figure A-15 Average Particle Concentrations of Test Sequence E01921 .................................. 193

Figure A-16 Average Particle Concentrations of Test Sequence E01977.................................. 194

Figure A-17 Average Particle Concentrations of Test Sequence E02099................................. 195

Figure A-18 Average Particle Concentrations of Test Sequence E02138 .................................. 196

Figure A-19 Average Particle Concentrations of Test Sequence E01959................................. 197

Figure A-20 PM Mass-Rate of E1920_02 as Measured with the TEOM................................... 198

Figure A-21 Corrected and Uncorrected PM Mass Accumulation of E01920_02 as Measured

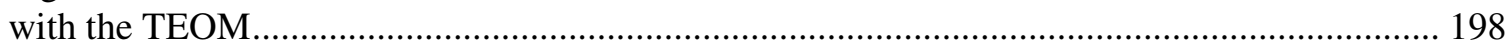

Figure A-22 PM Mass-Rates of E01920_02 as Measured with the TEOM and the DMS......... 199

Figure A-23 PM Mass Accumulation of E01920_02 as Measured with the TEOM and the DMS 199

Figure A-24 PM Mass-Rate of E01927_03 as Measured with the TEOM................................. 200 
Figure A-25 Corrected and Uncorrected PM Mass Accumulation of E01927_03 as Measured

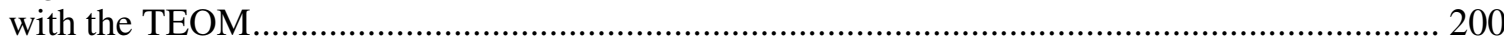

Figure A-26 PM Mass-Rates of E01927_03 as Measured with the TEOM and the DMS ......... 201

Figure A-27 PM Mass Accumulation of E01927_03 as Measured with the TEOM and the DMS 201

Figure A-28 PM Mass-Rate of E01929_04 as Measured with the TEOM................................ 202

Figure A-29 Corrected and Uncorrected PM Mass Accumulation of E01929_04 as Measured

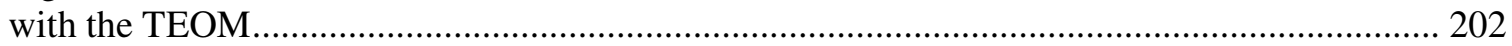

Figure A-30 PM Mass-Rates of E01929_04 as Measured with the TEOM and the DMS ......... 203

Figure A-31 PM Mass Accumulation of E01929_04 as Measured with the TEOM and the DMS

Figure A-32 PM Mass-Rate of E01967_04 as Measured with the TEOM................................... 204

Figure A-33 Corrected and Uncorrected PM Mass Accumulation of E01967_04 as Measured

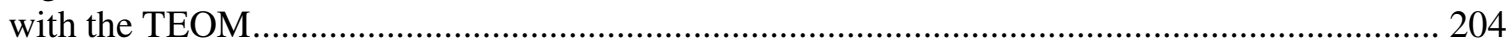

Figure A-34 PM Mass-Rates of E01967_04 as Measured with the TEOM and the DMS ......... 205

Figure A-35 PM Mass Accumulation of E01967_04 as Measured with the TEOM and the DMS

Figure A-36 PM Mass-Rate of E01968_04 as Measured with the TEOM.................................. 206

Figure A-37 Corrected and Uncorrected PM Mass Accumulation of E01968_04 as Measured

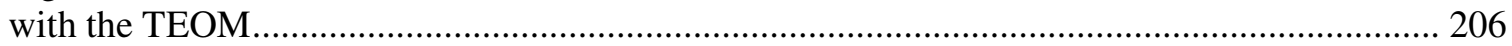

Figure A-38 PM Mass-Rates of E01968_04 as Measured with the TEOM and the DMS ......... 207

Figure A-39 PM Mass Accumulation of E01968_04 as Measured with the TEOM and the DMS

Figure A-40 PM Mass-Rate of E01976_04 as Measured with the TEOM................................. 208

Figure A-41 Corrected and Uncorrected PM Mass Accumulation of E01976_04 as Measured

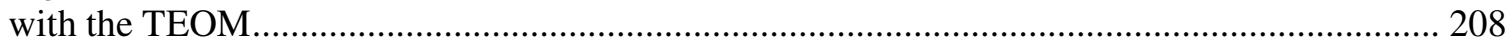

Figure A-42 PM Mass-Rates of E01976_04 as Measured with the TEOM and the DMS ......... 209

Figure A-43 PM Mass Accumulation of E01976_04 as Measured with the TEOM and the DMS

Figure A-44 PM Mass-Rate of E02124_02 as Measured with the TEOM................................ 210

Figure A-45 Corrected and Uncorrected PM Mass Accumulation of E02124_02 as Measured

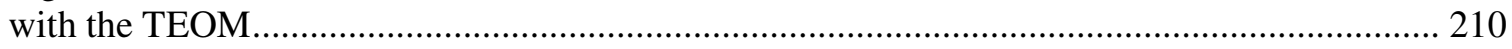

Figure A-46 PM Mass-Rates of E02124_02 as Measured with the TEOM and the DMS......... 211

Figure A-47 PM Mass Accumulation of E02124_02 as Measured with the TEOM and the DMS

Figure A-48 PM Mass-Rate of E02125_02 as Measured with the TEOM............................... 212

Figure A-49 Corrected and Uncorrected PM Mass Accumulation of E02125_02 as Measured

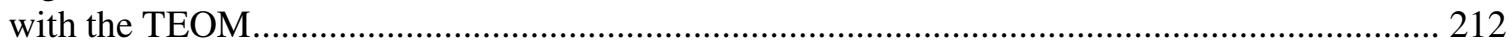

Figure A-50 PM Mass-Rates of E02125_02 as Measured with the TEOM and the DMS ......... 213 
Figure A-51 PM Mass Accumulation of E02125_02 as Measured with the TEOM and the DMS

Figure A-52 Particle Concentration Comparison of High, Medium, and Low Humidity Settings of Mode 2

Figure A-53 Particle Concentration Comparison of High, Medium, and Low Humidity Settings of Mode 5

Figure A-54 Particle Concentration Comparison of High, Medium, and Low Humidity Settings of Mode 6

Figure A-55 Particle Concentration Comparison of High, Medium, and Low Humidity Settings of Mode 7

Figure A-56 Particle Concentration Comparison of High, Medium, and Low Humidity Settings of Mode 10

Figure A-57 Particle Concentration Comparison of High, Medium, and Low Humidity Settings of Mode 11

Figure A-58 Particle Sizes that Have Significantly Different Average Values for Mode 3 Due to

Humidity Change (According to ANOVA).

Figure A-59 Particle Sizes that Have Significantly Different Average Values for Mode 4 Due to Humidity Change (According to ANOVA).

Figure A-60 Particle Sizes that Have Significantly Different Average Values for Mode 5 Due to Humidity Change (According to ANOVA).

Figure A-61 Particle Sizes that Have Significantly Different Average Values for Mode 6 Due to Humidity Change (According to ANOVA). 218

Figure A-62 Particle Sizes that Have Significantly Different Average Values for Mode 7 Due to Humidity Change (According to ANOVA).

Figure A-63 Particle Sizes that Have Significantly Different Average Values for Mode 8 Due to Humidity Change (According to ANOVA).

Figure A-64 Particle Sizes that Have Significantly Different Average Values for Mode 9 Due to Humidity Change (According to ANOVA). 220

Figure A-65 Particle Sizes that Have Significantly Different Average Values for Mode 10 Due to Humidity Change (According to ANOVA). 220

Figure A-66 Particle Sizes that Have Significantly Different Average Values for Mode 11 Due to Humidity Change (According to ANOVA). 221

Figure A-67 Particle Sizes that Have Significantly Different Average Values for Mode 12 Due to Humidity Change (According to ANOVA). 221

Figure A-68 Particle Concentration Comparison of High and Low Dilution Settings of Mode 2 223

Figure A-69 Particle Concentration Comparison of High and Low Dilution Settings of Mode 5 223

Figure A-70 Particle Concentration Comparison of High and Low Dilution Settings of Mode 6 
Figure A-71 Particle Concentration Comparison of High and Low Dilution Settings of Mode 7 224

Figure A-72 Particle Concentration Comparison of High and Low Dilution Settings of Mode 10 225

Figure A-73 Particle Concentration Comparison of High and Low Dilution Settings of Mode 11

Figure A-74 Continuous Particle Distribution of E02208_01 ................................................... 226

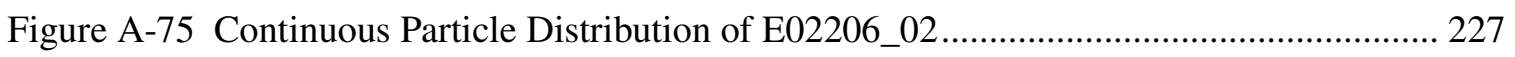

Figure A-76 Continuous Particle Distribution of E02208_02 ................................................ 228 


\section{LIST OF TABLES}

Table 1 US EPA Emissions Regulations for Heavy Duty Diesel Truck Engines ......................... 6

Table 2 Composition of Diesel Particulate Ash ......................................................................... 9

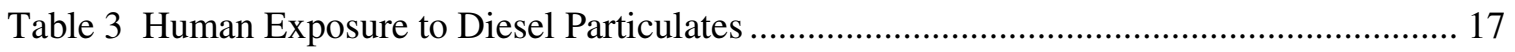

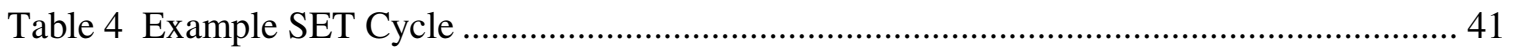

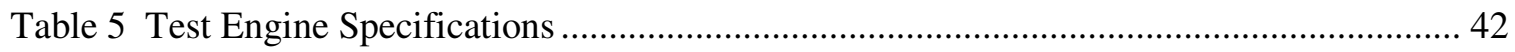

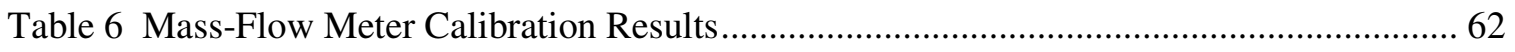

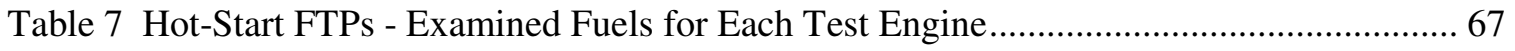

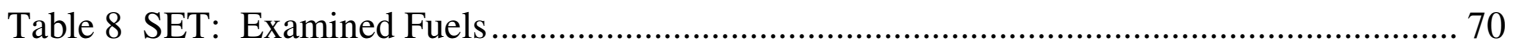

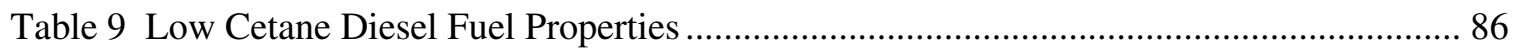

Table 10 Cetane Numbers and Additive Amounts of Low Cetane Fuels..................................... 87

Table 11 Differences in Average Particle Concentrations Due to Mixing of Additives with Low

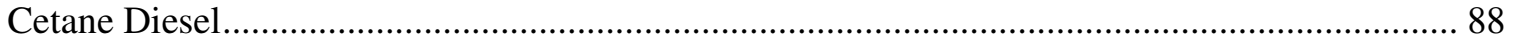

Table 12 COVs of Laboratory Measurements and DMS COV Scores ...................................... 101

Table 13 Water Absorption and Effective Density Coefficients ............................................... 102

Table 14 Total PM Mass Results of TEOM, DMS, and DPF ................................................. 116

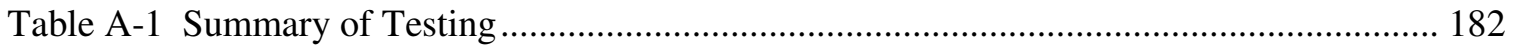




\section{LIST OF ACROYMNS AND ABBREVIATIONS}

AFEF

ADEF

CAFEE

CFR

$\mathrm{CFV}$

$\mathrm{CNC}$

$\mathrm{CO}$

$\mathrm{CO}_{2}$

$\mathrm{COV}$

CPC

CVS

CVS-EP

DAQ

DEMDT

DMA

DMPS

DPF

DPM

DR

ECU

EGR

ELPI

EMA

EPA

FCE

FTP

GC/MS

GVWR

HDDE

HEI

HEPA

HFID

HVAC

LAFY
Apparent Fuel Effect Factor

Apparent Dilution Effect Factor

Center for Alternative Fuels, Engines, and Emissions

Code of Federal Regulations

Critical Flow Venturi

Condensation Nucleus Counter

Carbon Monoxide

Carbon Dioxide

Coefficient of Variation

Condensation Particle Counter

Constant Volume Sampler

CVS combined with an Ejector Pump

Data Acquisition System

Double-Ejector Micro-Dilution Tunnel

Differential Mobility Analyzer

Differential Mobility Particle Spectrometer

Diesel Particulate Filter

Diesel Particulate Matter

Dilution Ratio

Engine Control Unit

Exhaust Gas Recirculation

Electrical Low Pressure Impactor

Electrical Mobility Analyzer

Environmental Protection Agency

Faraday Cup Electrometer

Federal Test Procedure

Gas Chromatograph / Mass Spectrometer

Gross Vehicle Weight Rating

Heavy-Duty Diesel Engines

Health Effects Institute

High Efficiency Particulate Air

Heated Flame Ionization Detector

Heating, Ventilating, and Air Conditioning

Los Angeles Freeway 


\begin{tabular}{ll} 
LANF & Los Angeles Non-Freeway \\
LC & Low-Cetane Reference Fuel \\
LDDE & Light Duty Diesel Engine \\
LFE & Laminar Flow Element \\
LII & Laser Induced Incandescence \\
LPI & Low Pressure Impactors \\
LRRI & Lovelace Respiratory Research Institute \\
MOUDI & Micro-Orifice Uniform Deposit Impactors \\
MPS & Micro Proportional Diluter \\
Nano-DMA & Nanometer Differential Mobility Analyzer \\
Nano-MOUDI & Nano-Micro-Orifice Uniform Deposit Impactors \\
n-ASA & Nanometer Aerosol Size Analyzer \\
NDIR & Non-Dispersive Infrared \\
NO & Oxides of Nitrogen \\
NYNF & New York Non-Freeway \\
O $_{3}$ & Ozone \\
PAH & Polynuclear Aromatic Hydrocarbons \\
PAS & Photoelectric Aerosol Sensor \\
PFDB & Parallel Flow Diffusion Battery \\
PID & Proportional-Integral-Derivative \\
PM & Particulate Matter \\
PTL & Particle Technology Laboratory \\
QCM & Quartz Crystal Microbalance \\
SET & Supplemental Emissions Test \\
SMPS & Scanning Mobility Particle Sizer \\
SO & Sulfate Particulates \\
SOF & Soluble Organic Fraction \\
SOL & Solid Fraction \\
TD & Thermodesorber \\
TEM & Transmission Electron Microscope \\
TEOM & Tapered Element Oscillation Microbalance \\
THC & Total Hydrocarbons \\
TPM & Total Particulate Matter \\
VOC & Volatile Organic Compounds \\
VGT & Variable Geometry Turbocharger \\
WOT & Wide Open Throttle \\
\hline &
\end{tabular}




\section{LIST OF SYMBOLS}

\begin{tabular}{|c|c|}
\hline $\mathrm{H}_{2} \dot{\mathrm{O}}_{\text {filter }}$ & Deposition Rate of Moisture onto the TEOM Filter \\
\hline$C_{1}$ & Mass Transfer Coefficient \\
\hline$C_{2}$ & Unitless Equilibrium Constant \\
\hline $\mathrm{H}_{2} \mathrm{O}_{\text {air }}$ & Moisture Mass in the Air \\
\hline $\mathrm{H}_{2} \mathrm{O}_{\text {fitter }}$ & Moisture Mass on the TEOM Filter \\
\hline$Q_{\text {tunnel }}^{\bullet}$ & Volumetric Flow Rate through the Primary Dilution Tunnel \\
\hline $\mathrm{CO}_{2, \text { tunnel }}$ & Mass Flow Rate of $\mathrm{CO}_{2}$ through the Primary Dilution Tunne \\
\hline$P M_{T E O M}$ & Total PM Mass Measured by the TEOM \\
\hline$P M_{\text {filter }}$ & Total PM Mass Measured by a Gravimetric Filter \\
\hline TEOM & Mass Flow Rate of $\mathrm{PM}$ and $\mathrm{H}_{2} \mathrm{O}$ given by the TEOM \\
\hline$P \dot{M}_{\text {filter }}$ & Mass Flow Rate of PM given by the TEOM \\
\hline$\Delta \mathrm{H}_{2} \mathrm{O}_{\text {filter }}$ & Change in Moisture Mass on the TEOM Filter \\
\hline$\Delta t$ & Change in Time \\
\hline$\omega_{\text {filter }}$ & Humidity Ratio for the TEOM Filter \\
\hline $\mathbf{M}_{\mathrm{H}_{2} \mathrm{O}}$ & Filter Moisture Mass \\
\hline $\mathbf{M}_{\text {filter }}$ & Net Filter Mass \\
\hline$\omega_{\text {air }}$ & Humidity Ratio for Air \\
\hline $\mathrm{M}_{\text {air }}$ & Mass of Air for a given Volume \\
\hline$\phi$ & Relative Humidity \\
\hline$p_{g}$ & Saturation Vapor Pressure \\
\hline$p$ & Barometric Pressure \\
\hline$R_{p}$ & Primary Dilution Ratio \\
\hline$R_{o}$ & Overall Dilution Ratio \\
\hline$R_{s}$ & Secondary Dilution Ratio \\
\hline$Q_{\text {total }}$ & Standardized Flow Rate in the Dilution Tunnel \\
\hline
\end{tabular}




\begin{tabular}{|c|c|}
\hline$Q_{\text {raw }}$ & Standardized Flow Rate in the Exhaust Pipe \\
\hline$C_{\text {venturi }}$ & Venturi Calibration Constant \\
\hline$p_{a b s}$ & Absolute Pressure \\
\hline$T_{a b s}$ & Absolute Temperature \\
\hline$e$ & Magnitude of Error Bar \\
\hline$e_{1}$ & Standard Deviation of Original Testing \\
\hline$e_{2}$ & Standard Deviation of Comparative Testing \\
\hline$a_{1}$ & Average Value of Original Testing \\
\hline COVscore & Weighted Sum of COV Values \\
\hline $\mathrm{COV}_{5-9}$ & Sum of COV Values between $5 \mathrm{~nm}$ and $9 \mathrm{~nm}$ \\
\hline$C O V_{10-19}$ & Sum of COV Values between $10 \mathrm{~nm}$ and $19 \mathrm{~nm}$ \\
\hline $\operatorname{COV}_{20-249}$ & Sum of COV Values between $20 \mathrm{~nm}$ and $249 \mathrm{~nm}$ \\
\hline$C O V_{250-499}$ & Sum of COV Values between $250 \mathrm{~nm}$ and $499 \mathrm{~nm}$ \\
\hline$C O V_{500-1000}$ & Sum of COV Values between $500 \mathrm{~nm}$ and $1000 \mathrm{~nm}$ \\
\hline$\rho_{\text {eff }}$ & Effective Particle Density \\
\hline$b_{1}$ & Effective Density Coefficient \\
\hline$b_{2}$ & Effective Density Coefficient \\
\hline$d$ & Equivalent Momentum Particle Diameter \\
\hline$\dot{m}$ & Mass Flow Rate for Particles of a given Diameter \\
\hline$C$ & Measured Particle Concentration \\
\hline$m$ & Particle Mass \\
\hline$\dot{M}$ & Total Mass Flow Rate of PM \\
\hline$Q$ & Volumetric Flow Rate \\
\hline$r$ & Radius \\
\hline$\pi$ & $\mathrm{Pi}$ \\
\hline$l$ & Length \\
\hline$C_{a}$ & Particle Concentrations of Additized Fuel \\
\hline$C_{b}$ & Particle Concentrations of Base Fuel \\
\hline
\end{tabular}




\section{INTRODUCTION}

\subsection{PROLOGUE}

After over 30 years of emissions regulations, tailpipe pollution from fossil fuel combustion is still threatening the environment and human health in the United States of America. The Environmental Protection Agency (EPA) claims that at least half of all Americans reside in areas that do not meet the basic standards of air quality. In major cities, vehicles are responsible for at least $50 \%$ of the total pollution and an even larger portion of the air pollution. Around $80 \%$ of the air-related cancer risk in some cities can be attributed to tailpipe emissions [6].

Particulate matter (PM) from heavy-duty diesel engines (HDDE) is one of several emissions that are considered harmful by the EPA. The wide variety of organic compounds, temperatures, and pressures that a diesel combustion mixture undergoes causes diesel engines, by nature, to emit greater amounts of particulates (on a mass per energy basis) than gasoline engines [2]. EPA regulations and the general need for greater combustion efficiency have forced diesel engine manufacturers to decrease total PM emission levels drastically. Since 1988, PM emission standards have decreased from 0.6 $\mathrm{g} / \mathrm{bhp}-\mathrm{hr}$ (1988) to the present standard of $0.01 \mathrm{~g} / \mathrm{bhp}-\mathrm{hr}$. Federal regulations currently do not consider particle size, and there are no set standards for size measurement of particles

emitted from internal combustion engines. Little is known about the direct effects of diesel particulate matter (DPM) on human health, but studies suggest that particles smaller than $50 \mathrm{~nm}$ have adverse effects if inhaled [3]. 
Due to physical changes that diesel particulates continuously undergo, DPM is not a well-defined chemical species [3]. In the existing literature, there is much uncertainty concerning the measurement results of DPM. For the underlying studies, DPM is the non-gaseous component emitted from raw diesel exhaust that has been diluted and cooled.

\subsection{OBJECTIVES}

This research was conducted in order to ascertain the minimum detectable variations in PM number concentrations for various conditions (objective 1). Specifically, emphasis was placed on varying the engine type, fuel type, relative humidity within the dilution tunnel, and primary dilution ratio. It was also aspired to determine the direct effects that humidity (objective 2) and dilution levels (objective 3) have on particle formation throughout various steady state operating conditions. In addition, it was elected to explore barometric pressure effects (objective 4); mainly because it was suspected that changes in barometric pressure could cause increased run-to-run or day-today variability in engine-out DPM. Beyond determining minimum significant differences, it was necessary to discover the overall effects that fuel type has on particle size distribution (objective 5). Another key focus was to relate continuous PM mass data from a Tapered Element Oscillating Microbalance (TEOM) to real-time particle size distribution data from a Cambustion DMS-500 (objective 6). Additionally, it was intended to study the effect of aerosol sample line temperature on nanoparticle formation (objective 7). The observable relationships between transient engine operating events and particle concentrations were also of interest (objective 8). 


\subsection{GOALS}

To accomplish the preceding objectives, there was a need to create and follow a multitude of exhaust sampling standards. To achieve a broad spectrum of results, it was decided to test three heavy-duty diesel engines and about 30 fuels on an engine dynamometer with a full scale dilution tunnel. To learn about the variations in particle size; having the ability to independently vary parameters such as engine type, fuel type, test type, humidity, sample line temperature, and dilution ratio was essential. The transient Federal Test Procedure (FTP) was used in order to simulate on-road operating conditions; but use of the Supplemental Emissions Test (SET) was also necessary for discriminating subtle differences in test repeatability and particle size distribution due to changes in relative humidity or dilution ratio during steady-state operation. To obtain a rounded understanding of variation, it was required to examine residence time, sampling position, and equipment used. To use the selected PM sampling instrument, the DMS500 , it became evident that it was essential to build a suitable secondary dilution system. The secondary system had to be capable of diluting the primary aerosol sample at a ratio of about 25 to 1 and maintaining an internal temperature of about $47 \mathrm{deg} \mathrm{C}$. 


\section{LITERATURE REVIEW}

\subsection{BACKGROUND}

Early DPM research, in 1976, was conducted at Michigan Technological University by Vuk et al. The goal of the research was a better understanding of particle size and concentration, and the research was done by passing exhaust through multiple metal plates, each with hundreds of small holes ranging from $6.05 \mu \mathrm{m}$ to $0.53 \mu \mathrm{m}$. When a test was over, the number of plugged holes on a given plate was estimated to be the total number of a single sized particle for the entirety of the test. The total number divided by the total test flow resulted in a concentration value [4]. Since 1976, much technical advancement has been made toward the accuracy and reliability of DPM size and concentration measurements. Today, there are many available types of equipment used to measure DPM. They are designed to measure total mass, total concentration, concentration of a specific size, and concentrations of sizes in a particular range. DPM sampling instruments include, but are not limited to, the: fast-scanning nanometer Aerosol Size Analyzer (n-ASA), Differential Mobility Analyzer (DMA), Differential Mobility Particle Spectrometer (DMPS), Transmission Electron Microscope (TEM), Tapered Element Oscillating Microbalance (TEOM), Quartz Crystal Microbalance (QCM), Thermodesorber (TD), Laser Induced Incandescence (LII), Photoelectric Aerosol Sensor (PAS), Gravimetric Particulate Filter, DMS-500 fast particle sizer, smoke meter, Faraday Cup Electrometer (FCE), Condensation Nucleus Counter (CNC), Cascade Impactor (CI), Electrical Low Pressure Impactor (ELPI), and the Scanning Mobility Particle Sizer (SMPS). Each instrument has certain advantages and disadvantages that involve applicability, accuracy, precision, response time, size, and weight. With the 
assortment of manufacturing companies come different particle size equivalence definitions. Many diesel particulates are agglomerates of carbon and other solid material, and they are more chainlike in shape than spherical [5]. Particle sizing instruments convert readings into equivalent aerodynamic diameter or equivalent momentum diameter. Conversion techniques are tailored, causing results between sampling instruments to vary.

Many areas of research on DPM are available. DPM can be sampled from endless combinations of fuels, engines, after treatment devices, and locations. DPM can also be compared to particulate matter from other combustion equipment that can be used in similar applications as diesel engines. Some studies have even compared DPM to particulate matter formed by burning wood [2].

\subsection{EPA STANDARDS AND REGULATIONS}

\subsubsection{Standards of Measurement}

For 2007 and newer HDDE dynamometer certification, the Code of Federal Regulations (CFR) states that a proportional sample is to be taken from the primary dilution tunnel and further diluted by means of a secondary dilution tunnel. The primary/secondary dilution proportion must be maintained within $\pm 5 \%$, excluding startup. The double-diluted sample is to be passed through a fibrous collection filter, or gravimetric filter. The secondary dilution air must be greater than or equal to $15^{\circ} \mathrm{C}$, and the final sample must be maintained at $47^{\circ} \mathrm{C} \pm 5{ }^{\circ} \mathrm{C}$. The face velocity into the sample filter is not to exceed $100 \mathrm{~cm} / \mathrm{s}$. Specific filters, such as polytetraflouroethylene coated borosilicate glass fiber high-efficiency filters or polytetraflouroethylene high-efficiency membrane filters with polymethylpentene integral support rings are required. The filters 
must have a diameter of $46.5 \mathrm{~mm} \pm 0.6 \mathrm{~mm}$ and a clean filter efficiency of at least $99 \%$ as measured by the ASTM D2986-95a DOP test. The filter holder assembly is to be made of 302, 303, or 304 stainless steel. Sampling standards can be found in greater detail in the CFR (Title 40, Part 86, Subpart N) [1].

\subsubsection{Regulations}

Until 1988, particulate matter was not regulated in the United States. Since 1988, on-road HDDE PM regulations have become dramatically more stringent. The first regulation on PM, set by the EPA, was $0.60 \mathrm{~g} / \mathrm{bhp}-\mathrm{hr}$. In 1991, the standard was updated to $0.25 \mathrm{~g} / \mathrm{bhp}$-hr. In 1994 , it was changed to $0.10 \mathrm{~g} / \mathrm{bhp}-\mathrm{hr}$. The most recent change was made in 2007, which was from $0.10 \mathrm{~g} / \mathrm{bhp}-\mathrm{hr}$ to $0.01 \mathrm{~g} / \mathrm{bhp}-\mathrm{hr}$ [1]. Below in Table 1 are the United States EPA emissions regulations for on-road Heavy-Duty Diesel Engines.

Table 1 US EPA Emissions Regulations for Heavy Duty Diesel Truck Engines (g/bhp-hr)

\begin{tabular}{|c|c|c|c|c|c|c|}
\hline Year & HC & CO & NOx & PM & NMHX + NOx & NMHC \\
\hline 1988 & 1.3 & 15.5 & 10.7 & 0.6 & n/a & n/a \\
\hline 1990 & 1.3 & 15.5 & 6 & 0.6 & n/a & n/a \\
\hline 1991 & 1.3 & 15.5 & 5 & 0.25 & n/a & n/a \\
\hline 1994 & 1.3 & 15.5 & 5 & 0.1 & $\mathrm{n} / \mathrm{a}$ & $\mathrm{n} / \mathrm{a}$ \\
\hline 1998 & 1.3 & 15.5 & 4 & 0.1 & $\mathrm{n} / \mathrm{a}$ & $\mathrm{n} / \mathrm{a}$ \\
\hline $2004^{*}(1)$ & 1.3 & 15.5 & $\mathrm{n} / \mathrm{a}$ & 0.1 & 2.4 & $\mathrm{n} / \mathrm{a}$ \\
\hline $2004(2)$ & 1.3 & 15.5 & $\mathrm{n} / \mathrm{a}$ & 0.1 & 2.5 & 0.5 \\
\hline $2007^{\#}$ & 1.3 & 15.5 & 0.2 & 0.01 & $\mathrm{n} / \mathrm{a}$ & 0.14 \\
\hline
\end{tabular}

* 2004 standards in effect October 2002

\# Phase in period to 2010

PM is not the only constituent that has been targeted for reduction. The regulation for oxides of nitrogen, or $\mathrm{NO}_{\mathrm{x}}$, has been reduced from $10.7 \mathrm{~g} / \mathrm{bhp}-\mathrm{hr}$ (1988) to the current standard of $0.2 \mathrm{~g} / \mathrm{bhp}-\mathrm{hr}$. Regulations are becoming increasingly strict for several reasons. For example, $\mathrm{NO}_{\mathrm{x}}$ is a key ingredient for smog, and $\mathrm{PM}$ has been linked to several pollution problems, such as acid rain. The regulation for $\mathrm{NO}_{\mathrm{x}}$ is not shown in 
Table 1 , because in 2004, the regulation was for the brake-specific summation of nonmethane hydrocarbons (NMHC) and $\mathrm{NO}_{\mathrm{x}}$.

\subsection{PARTICLE TRAITS}

\subsubsection{Composition}

DPM is a complex diesel emission that consists of solids and liquid material that condenses during dilution. DPM is composed of three fundamental fractions: solid fraction $(\mathrm{SOL})$, soluble organic fraction $(\mathrm{SOF})$, and sulfate particulates $\left(\mathrm{SO}_{4}\right)$. $\mathrm{SOL}$ particles consist of elemental carbon and ash. SOF particles include organic material derived from engine oil and organic components derived from fuel, and $\mathrm{SO}_{4}$ particles are mainly a combination of sulfuric acid and water. The sum of $\mathrm{SOF}$ and $\mathrm{SO}_{4}$ particles can be referred to as the volatile organic compounds (VOC). Figure 1 illustrates the composition of DPM from a HDDE engine during a US FTP transient cycle. Note that the fractions of each constituent generally vary with engine type and load [2].

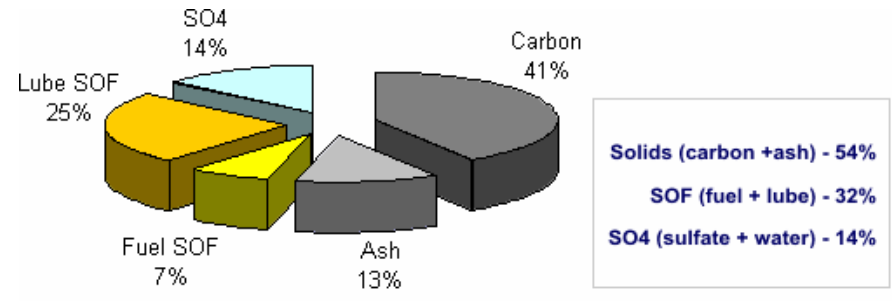

Figure 1 Composition of Diesel Particulate Matter [2]

Total particulate matter (TPM) can be defined as:

$$
\mathrm{TPM}=\mathrm{SOL}+\mathrm{SOF}+\mathrm{SO}_{4} \quad \text { Equation } 1
$$

There are two major size ranges, known as nuclei mode and accumulation mode, used to describe DPM. Nuclei mode is between $0.007 \mu \mathrm{m}$ and $0.04 \mu \mathrm{m}$, and it consist primarily of volatile particles that are very unstable. Volatile particles are hydrocarbon 
and hydrated sulfuric acid condensates that depend on dilution conditions. A small fraction of nuclei mode particles can include elemental carbon or metallic ash. Nuclei mode particles usually make up at least $90 \%$ of the total particle count, but only a few percent of the total mass. Accumulation mode particles range from $0.04 \mu \mathrm{m}$ to $1 \mu \mathrm{m}$, and are composed primarily of solid carbon particles mixed with condensed heavy hydrocarbons. Accumulation mode can also include metallic ash, sulfur compounds, and cylinder wear metals. Over $95 \%$ of total DPM mass is generally attributed to accumulation mode particles. Figure 2, below, provides a graphical representation of the composition of nuclei mode and accumulation mode particles. Accumulation mode consists mostly of solid particles formed during combustion, while nuclei mode consists of volatile particles formed during cooling and dilution.
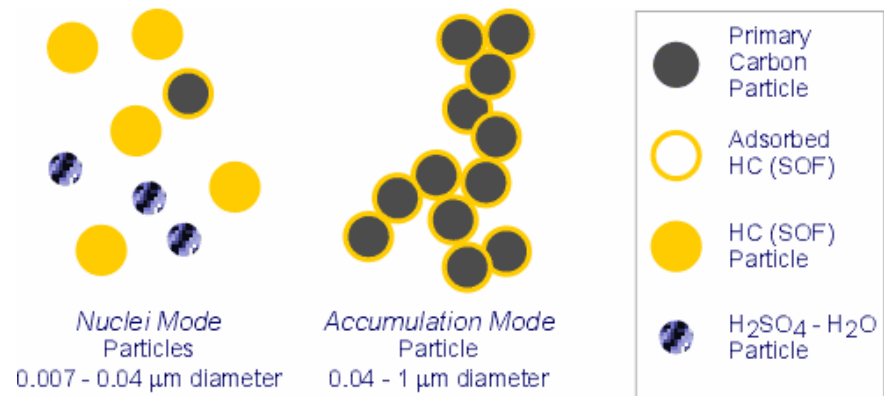

\section{Figure 2 Basic Composition of Nuclei Mode and Accumulation Mode Particles [2]}

A large portion of the polynuclear aromatic hydrocarbons (PAH) found in diesel exhaust is carried by DPM. Most PAHs exist in the $\mathrm{SO}_{4}$ fraction. Dioxins are chlorinated polynuclear hydrocarbon compounds that are classified as $\mathrm{SO}_{4} \mathrm{PAHs}$ and are characterized by their toxicity, carcinogenicity, and resistance to breakdown. Shown in Figure 3, TCDF is a dioxin found in DPM that is considered highly toxic. Diesel engines create TCDFs by breaking down motor oil. There are certain types of catalytic 
combustion additives that increase the emissions of dioxins by orders of magnitude [2]. With TCDFs, traces of chlorine derived from engine oil form sturdy symmetrical bonds with oxygen atoms.

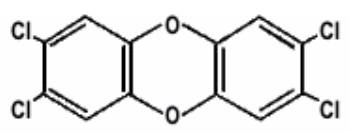

2,3,7,8-Tetrachlorodibenzo-p-dioxin

Figure 3 Example Dioxin Compound TCDF [2]

Engine ash is made up of sulfates, phosphates, and metal oxides. Ash particles can agglomerate to form primary particles of up to $500 \mathrm{~nm}$ in size. Some examples of engine ash composition are represented in Table 2.

Table 2 Composition of Diesel Particulate Ash [2]

\begin{tabular}{|c|c|c|c|c||}
\hline \multirow{2}{*}{ Material } & Ash A & \multicolumn{2}{|c|}{ Ash B } & \multicolumn{2}{|c|}{ Ash C } & Ash D \\
\cline { 2 - 4 } & \multicolumn{2}{|c|}{ |eight percent of oxide in ash } \\
\hline \hline $\mathrm{CaO}$ & 29.6 & 20.7 & 10.4 & 27.6 \\
\hline \hline $\mathrm{ZnO}$ & 9.9 & 6.9 & 3.5 & 8.22 \\
\hline \hline $\mathrm{MgO}$ & 5.5 & 3.9 & 1.9 & 1.66 \\
\hline \hline $\mathrm{SO}_{3}$ & 38.8 & 27.2 & 13.6 & - \\
\hline \hline $\mathrm{P}_{2} \mathrm{O}_{5}$ & 15.8 & 11.1 & 5.5 & 10.4 \\
\hline \hline $\mathrm{Al}_{2} \mathrm{O}_{3}$ & - & - & - & 1.28 \\
\hline \hline $\mathrm{SiO}_{2}$ & - & - & - & 3.81 \\
\hline \hline $\mathrm{Cr}_{2} \mathrm{O}_{3}$ & - & - & - & 0.14 \\
\hline \hline $\mathrm{CuO}_{2}$ & - & - & - & 0.22 \\
\hline \hline $\mathrm{Fe}_{2} \mathrm{O}_{3}$ & 0.41 & 30.3 & 0.14 & 1.57 \\
\hline \hline $\mathrm{CeO}_{2}$ & 0.0 & 0.0 & 65.0 & - \\
\hline \hline
\end{tabular}

The data was collected using a diesel particulate filter (DPF) over a 100,000 km HDDE experiment. Ashes A, B, C, and D were created by combining different fuel additives and after treatment devices. Generally, if a metal-based fuel additive is used, engine ash contains oxides of the metal from within the additive. Metals often used in 
additives are strontium, iron, and cerium. Composition of DPM varies and depends greatly upon engine technology, test conditions, and sulfur content in the fuel [2]. There is much literature where in-depth research was performed on these topics.

\subsubsection{Formation}

The US EPA specifies that DPM mass is to be sampled at exhaust temperatures no higher than $52{ }^{\circ} \mathrm{C}$. Laboratory systems known as dilution tunnels are used to achieve such temperatures, as well as dilution ratios generally ranging from 2 to 20 . The purpose of cooling and diluting raw exhaust is to simulate the formation of DPM in the atmosphere. Since atmospheric dilution ratios of DPM are often in the hundreds or thousands near the exit of the exhaust, it is extremely complicated to precisely simulate natural dilution. The formation of nuclei mode particles depends strongly on dilution conditions such as temperature, humidity, dilution ratio, and residence time. In accumulation mode, the formation of agglomeration particles is also related to dilution conditions. Nuclei mode and agglomeration particles are affected by dilution conditions because of the absorption of gases and condensation of vapors.

Depending on temperature, particles undergo specific amounts of oxidation and agglomeration. Some particles are deposited on the inside of an exhaust pipe because of thermophoretic forces (mass transfer driven by a temperature gradient). Initially present as hot gases, exhaust constituents involved in latter agglomeration are hydrocarbons, sulfur oxides, and water. Solid ash is derived from lubrication oil, fuel additives, and normal engine wear. Volatile ash is formed from nucleation of ash constituents during the expansion stroke. Heavy hydrocarbons are derived from oil and unburned fuel. The organic portion of SOF is formed when the surfaces of carbon particles absorb heavy 
hydrocarbons. If the available sponge-like carbon particles are insufficient, hydrocarbons may nucleate to form increased numbers of volatile nuclei mode particles. In theory, total hydrocarbon material from the combustion chamber is proportional to particulate SOF and gas phase hydrocarbons. Sulfuric acid in DPM is derived from the sulfur content of fuel and oil. Water can react with $\mathrm{SO}_{3}$ to produce sulfuric acid, or $\mathrm{H}_{2} \mathrm{SO}_{4}$. $\mathrm{SO}_{3}$, or sulfur trioxide, is formed in the combustion chamber by the oxidation of sulfur. Only a small portion of sulfur in the combustion mixture forms $\mathrm{SO}_{3}$, because $\mathrm{SO}_{2}$, or sulfur dioxide, ties up about $95 \%$ of the available sulfur. A heteronucleation process between sulfuric acid and water is responsible for the formation of $\mathrm{SO}_{4}$ particulates. Sulfuric acid may also form SOL sulfate salts, depending on the availability of metal based compounds. Figure 4 illustrates how relative humidity in the surrounding atmosphere is largely responsible for the formation of sulfuric acid.

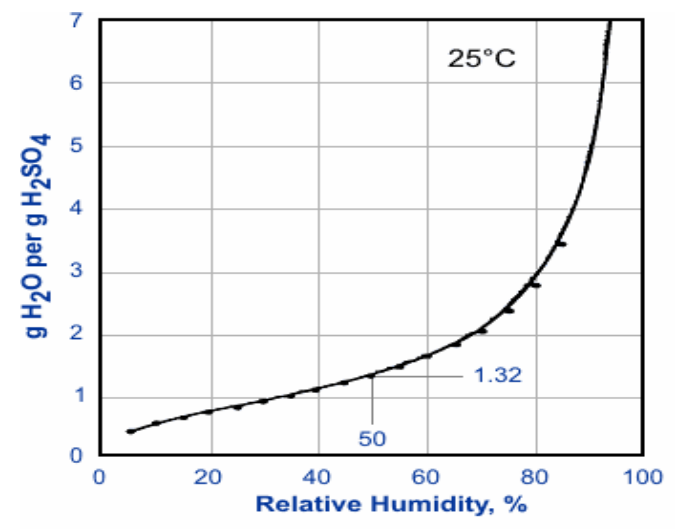

Figure 4 Mass of Bound $\mathrm{H}_{2} \mathrm{O}$ per Gram of $\mathrm{H}_{2} \mathrm{SO}_{4}$ [3]

Elemental carbon, or inorganic carbon, makes up the majority of SOL particles. Not chemically bound with other emissions, inorganic carbon is responsible for black smoke emissions. Figure 5 shows how carbon atoms form hexagonal arrays, or platelet shaped structures that are arranged in two to five layer deep graphite crystallites. The crystallites generally pack themselves parallel to the surface of a primary particle. 


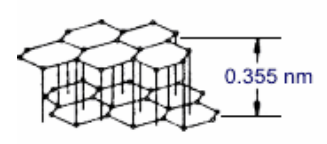

Platelet

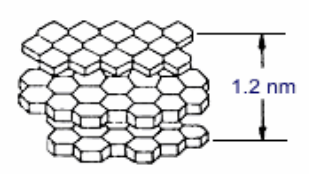

Platelets

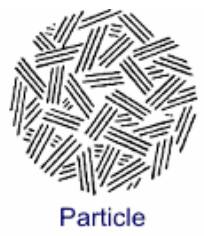

Particle

Figure 5 Structure of a Primary Elemental Carbon Particle [3]

Primary carbon particles agglomerate in the cylinder and change little until they are discharged into the atmosphere. The final form of part of an agglomerated particle is shown below in Figure 6. A nuclei mode primary particle usually agglomerates with hundreds of others to form a much larger accumulation mode particle that is grape-like in structure. Since the final elemental carbon particle is shaped rather like a chain, a sphere is a poor approximation of shape.

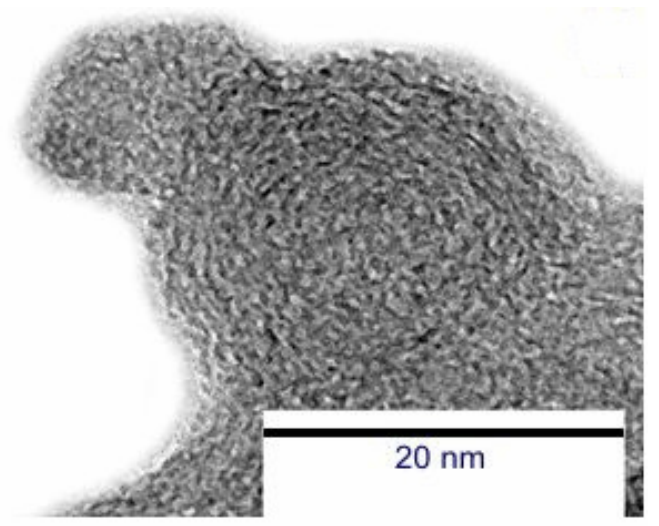

\section{Figure 6 TEM Picture of a Primary Carbon Particle Attached to an Agglomerated Carbon Particle [3]}

The SOL mass fraction is generally about $54 \%$ of DPM. In cases where the SOF fraction is greater than $50 \%$, DPM is called "wet" particulate. In wet particulates, multilayer hydrocarbon absorption on the surface of carbon particles takes place. Dry particulates, on the other hand, can have SOF content as low as $10 \%$. As seen in Figure 7, engine loads and exhaust temperatures are prominent variables that impact the formation of SOF particulates. 


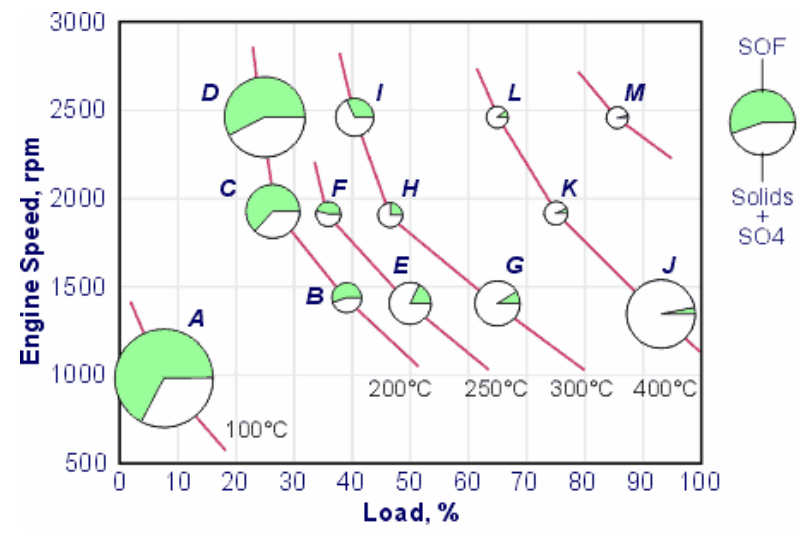

Figure 7 SOF Fractions at Different Operating Conditions [3]

\subsubsection{Size and Number}

The medical community has sparked an interest in the size distribution of DPM.

Indications have been made that adverse health effects increase when particle size decreases, even for toxicologically inert material. The increased interest of particle size was triggered by a study funded by the Health Effects Institute (HEI) [3]. The study, involving two HDDEs, showed that there was an increased number of nanoparticles from a newer engine; possibly due to higher injection pressures. PM is generally divided into four categories based on equivalent aerodynamic diameter. $\mathrm{PM}_{10}$ includes all particulates of aerodynamic diameter less than $10 \mu \mathrm{m}$. The $\mathrm{D}_{50}$ cut size is considered to be $10 \mu \mathrm{m}$, meaning that about $50 \%$ of the respirable particles are smaller than $10 \mu \mathrm{m}$. Fine particles are of aerodynamic diameter less than $2.5 \mu \mathrm{m}$. Ultrafine particles are of diameters less than $100 \mathrm{~nm}$, and nanoparticles are characterized by diameters less than 50 nm. There are also modal size classifications that are used to describe typical PM mass and number distributions. Coarse mode includes any particle with a diameter greater than $1 \mu \mathrm{m}$. Coarse mode typically contains $5 \%$ to $20 \%$ of the total mass, and practically $0 \%$ of the total particle count. Extending through fine, ultrafine, and part of the nanometer range, accumulation mode contains sub-micron particles ranging from $40 \mathrm{~nm}$ to $1000 \mathrm{~nm}$. 
Nuclei mode includes all particles between $7 \mathrm{~nm}$ and $40 \mathrm{~nm}$. A typical shift from an accumulation mode distribution to a nuclei mode distribution, as measured by a DMS500, is shown below by Figure 8 .

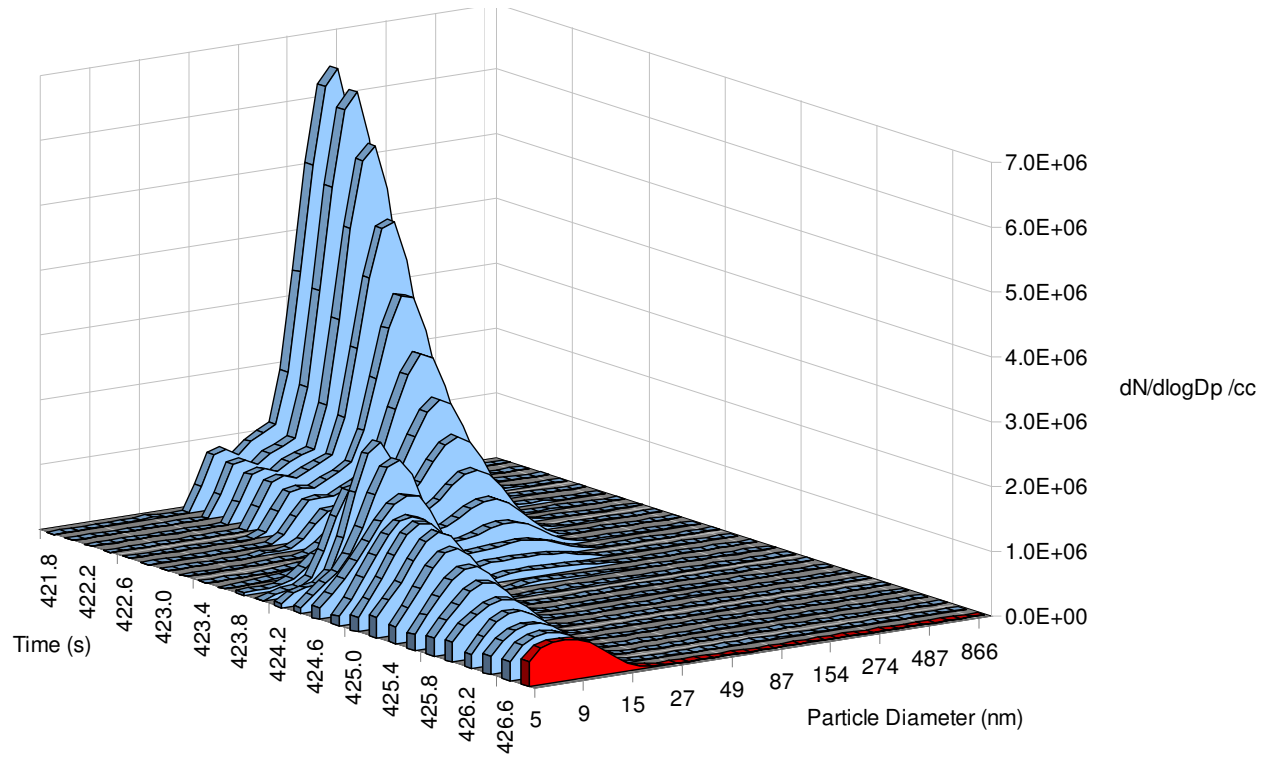

Figure 8 Nuclei Mode and Accumulation Mode

A study to compare the particle number emissions between diesel and gasoline engines was conducted by CONCAWE [3]. Using a matrix of diesel and gasoline fuels, four diesel vehicles and three gasoline vehicles were tested under different constant speed conditions using the ECE+EUDC test cycle. PM number results are shown in Figure 9. Note that under most circumstances, the number of diesel particles was one to three orders of magnitude higher than the number of gasoline particles. 


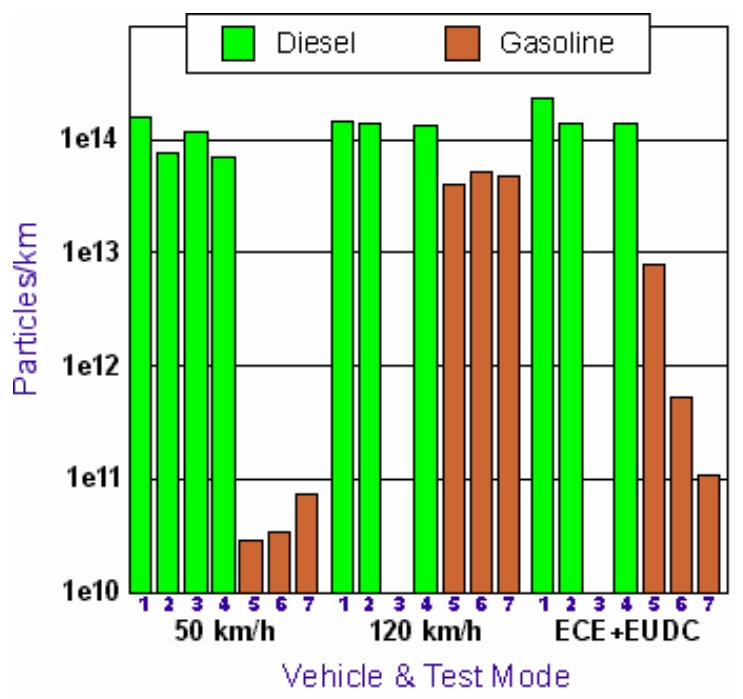

\section{Figure 9 Particulate Number Emissions from Diesel and Gasoline Vehicles [3]}

Residence time is the amount of time that particles spend in the dilution environment. Residence time's effect on DPM number and size has to do with the amount of time that it takes for condensation and particle nucleation to take place. Figure 10, below, illustrates a typical DPM number and size distribution when residence time is taken into account. It is clear that residence time only affects particle numbers and sizes for particles that are smaller than about $50 \mathrm{~nm}$. As illustrated in this figure, the nuclei mode particles are strongly influenced by residence time.

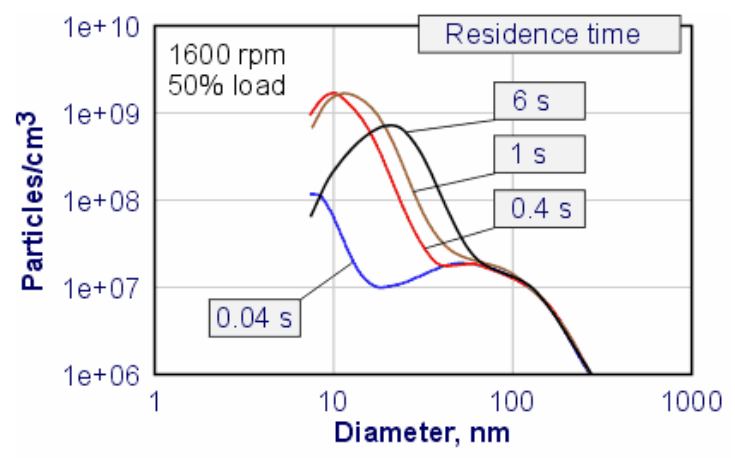

Figure 10 Effect of Residence Time on Particle Number and Size [3] 


\subsection{BIOLIGICAL CONCERNS}

Laboratory studies suggest that DPM includes several carcinogens, but the only proven health problems associated with DPM occur in the respiratory system [8]. Breathing air that contains diesel exhaust is the most common way in which people are affected by DPM. The level of penetration of DPM into the human body is dictated by particle size and residence time in the air. Particles larger than $100 \mu \mathrm{m}$ are deposited only in the nose and throat, while fine particles can be deposited in the alveoli or even pass through the lungs into the bloodstream and be transported to all parts of the body [8]. DPM affects health in different ways depending on levels of exposure.

Acute exposure (short term) to DPM can cause irritation to the eyes, nose, throat, and lungs. It can also exacerbate asthma, initiate coughing fits, or cause neurological effects such as nausea and lightheadedness. Symptoms of acute exposure are similar to those of seasonal allergies. Chronic exposure (occupational) causes lung inflammation and may have immunological effects [8].

Concentration of DPM in the ambient air varies significantly with location. Exposure can be measured in terms of concentration, and is generally given in units of $\mu \mathrm{g} / \mathrm{m}^{3}$. People are exposed in both leisure and occupational settings. An example of a low risk area is a rural area of low population and minimal traffic density. Some of the highest levels of occupational exposure are found in dieselized underground mines. Table 3 gives approximate ranges of exposure to DPM in several environments [9]. Concentrations in the urban areas were calculated from a monthly average, and concentrations in the occupational locations were measured. 
Table 3 Human Exposure to Diesel Particulates [9]

\begin{tabular}{|c|c|}
\hline Environment & Range of Exposure \\
\hline \multicolumn{2}{|l|}{ Urban areas } \\
\hline Less polluted (monthly average) & $1-4 \mu \mathrm{g} / \mathrm{m}^{3}$ \\
\hline Heavily polluted (monthly average) & $10 \mu \mathrm{g} / \mathrm{m}^{3}$ \\
\hline \multicolumn{2}{|l|}{$\begin{array}{r}\text { Occupational groups } \\
\end{array}$} \\
\hline Truck drivers & $4-6 \mu \mathrm{g} / \mathrm{m}^{3}$ \\
\hline Bus garage workers, forklift operators,.. & $20-100 \mu \mathrm{g} / \mathrm{m}^{3}$ \\
\hline $\begin{array}{ll}\text { Underground miners } \\
\end{array}$ & $100-1,700 \mu \mathrm{g} / \mathrm{m}^{3}$ \\
\hline
\end{tabular}

To decrease risk, a driver or operator can avoid excessive idling by turning the engine off at long pauses. Retrofitting a diesel engine with pollution control devices and keeping the engine well tuned and maintained can help to decrease DPM concentration in the immediate environment. In areas of very high concentration, wearing a mask is a practical method of reducing exposure [9].

\subsection{AVAILABLE SAMPLING EQUIPMENT}

There are numerous sampling methods for DPM size and distribution. In order to acquire comparable and conclusive data, sampling parameters must be consistent or analogously varied from test to test. Important sampling parameters such as: temperature, dilution ratio, residence time, relative humidity, and location can alter the formation of volatile particulates. Sampling location and dilution ratios are parameters that must be matched with their respective instruments. Listed below are some, but not all, of the available types of aerosol sampling equipment.

\subsubsection{Nanometer Aerosol Size Analyzer (n-ASA)}

The nanometer Aerosol Size Analyzer combines a bipolar charger $\left(\mathrm{Po}^{210}\right)$, an extended-length Nanometer Differential Mobility Analyzer (Nano-DMA), and an 
electrometer [10]. The result is an instrument that has a fast response and is capable of scanning 30 size channels between $3 \mathrm{~nm}$ and $100 \mathrm{~nm}$ in less than 3 seconds. The n-ASA is applicable where high concentrations exist and a broad range of temperatures and pressures is unavoidable. Transient nanoparticle analysis can be achieved with the nASA for dilution ratios between 5 and 30 [10].

\subsubsection{Differential Mobility Analyzer (DMA)}

The Differential Mobility Analyzer uses bipolar diffusion charging [11]. Particles are charged to an equilibrium energy level and passed through an Electrical Mobility Analyzer (EMA). The EMA only allows particles of a user-specified charge to pass through and be measured downstream. The electrical mobility, or charge, of a particle is directly proportional to the particle's mass. The DMA can be combined with other types of instruments in order to measure concentrations of more than one particle size simultaneously. If a sample is measured by a DMA and a Photoelectric Aerosol Sensor (PAS), results can be manipulated in order to approximate particle concentration and actual surface area. This is possible because the PAS gives particle diameter based on size, while the DMA gives diameter based on mass and aerodynamic drag [11].

\subsubsection{Differential Mobility Particle Spectrometer (DMPS)}

The Differential Mobility Particle Spectrometer operates by applying a stepwise voltage increase to a Differential Mobility Analyzer (DMA). At each voltage level, a specific interval of electric mobility particles can pass through the instrument and be measured. It takes a considerable amount of time to scan through particle intervals. To accomplish a full size distribution, at least 20 minutes of steady-state engine operation is required. More than one DMPS may be used during a single test to provide a greater 
coverage of particle distribution. The DMPS can be used with overall dilution ratios of between 5 and 30 [12].

\subsubsection{Transmission Electron Microscope (TEM)}

Much like a slide projector, the Transmission Electron Microscope shines a beam of electrons (instead of light) through a specimen. Electrons that pass through the specimen are transmitted onto a phosphor screen. Light is generated when electrons strike the phosphor, and three to five projector lenses are used to enlarge the image. Lighter areas of the final image represent thinner or less dense parts of the specimen, and dark areas represent thick or dense portions. TEMs can be used to determine the size and morphology of individual diesel exhaust particles. Particles can be taken from different points along the exhaust system in order to study the evolution of DPM along the exhaust stream [13].

\subsubsection{Tapered Element Oscillating Microbalance (TEOM)}

The Tapered Element Oscillating Microbalance is capable of measuring instantaneous mass flow of DPM. Particle mass is determined by the change in frequency of an oscillating tapered element as exhaust gases pass through it and onto an attached filter. The TEOM can be used to measure ambient air PM with a detection limit of $2 \mu \mathrm{g} / \mathrm{m}^{3}$ for a 24 hour integrated sample. It can also be used with diluted exhaust to give a continuous reading of DPM total mass flow [14]. The TEOM is not normally used in on-road emissions testing because of its sensitivity to condensation and vibration. 


\subsubsection{Quartz Crystal Microbalance (QCM)}

Unlike the TEOM, the Quartz Crystal Microbalance is not sensitive to condensation and external vibration. The QCM operates under the same principle as the TEOM, except instead of using a vibrating tapered element with a filter; it uses an oscillating quartz crystal and electrostatic precipitation. The QCM can be used for in-use emissions testing because of its compact size and resilience to vibration. It measures instantaneous DPM mass flow, and is generally used in unison with a Micro Proportional Diluter (MPS). Valid dilution ratios are between 2 and 15 [14].

\subsubsection{Thermodesorber (TD)}

The thermodesorber collects samples on multi-level sorption tubes. The tubes are connected to a Gas Chromatograph/Mass Spectrometer (GC/MS). High-resolution capillary chromatography separates chemicals. The chemicals identified as VOCs are quantified using toluene as an external standard. Target compounds are specified by the user. The TD can be used to measure levels of specified VOCs in ambient air. It can also be used along with other DPM instruments to determine the mass fraction of VOCs in a given sample [15].

\subsubsection{Laser-Induced Incandescence (LII)}

Laser-Induced Incandescence is a phenomenon that occurs when a laser beam encounters particulate matter. The absorption of energy causes soot particle temperatures

to increase. Particles then lose heat energy to immediate surroundings. Pre and posttemperature measurements are made to give an indication of energy absorption rate. Energy absorption rate of the exhaust flow is converted into soot volume fraction of DPM in the exhaust flow. Careful interpretation leads to primary particle size of soot 
agglomerates. Certain LII instruments can be used in the combustion chamber to obtain temporally resolved 2-D soot images at various crank angles and spatial locations [16].

\subsubsection{Photoelectric Aerosol Sensor (PAS)}

In the Photoelectric Aerosol Sensor, aerosols enter a quarts tube and are submitted to high-intensity UV light. The UV exposure causes particles to emit electrons which are collected in an ion trap. The resulting positively charged particles are collected on an electrically sensitive filter where their charge is measured by an electrometer. It is believed that the photoelectric effect is triggered by PAHs within DPM. For this reason, the PAS signal is generally related to PAH concentration. It is possible to use the PAS with a rotating-disk diluter to provide information on the composition of nanoparticles in diesel exhaust [14].

\subsubsection{DMS-500 Fast Particle Sizer}

The DMS-500 is considered to be an "inside-out" DMA. Aerosol passes through a charging chamber that assigns appropriate electrical charges to particles of various mass. A greater mass corresponds to a grater charge. The charged particles then enter a compartment that houses a high-voltage electrode and multiple electrometers. Each electrometer is designed to attract particles of a specific charge/drag ratio. The charges measured by an electrometer at any given time represent the particle concentration of a certain DPM size. The larger particles generally travel farther in the sheath air stream and make contact with the latter electrometer rings. There are over 20 electrodes. This allows the DMS-500 to measure size and concentration of DPM in real time. Through interpolation, the DMS-500 approximates the concentrations of 43 DPM sizes within the range of $5 \mathrm{~nm}$ to $1000 \mathrm{~nm}$. It can measure multiple sizes simultaneously, which makes it 
useful in transient HDDE dynamometer testing. The DMS-500 can also be used to determine how particle sizes and concentrations change during steady-state dynamometer tests [17]. The DMS-500 is limited to high dilution ratio cases (above 50) because it must be taken apart and cleaned when the electrometers become dirty [14].

\subsubsection{Smoke Meter}

By using a light passed through an exhaust stream and a light sensor on the other side; smoke meters, or opacity meters, detect the amount of light blocked by diesel exhaust. Smoke meters measure smoke density. In diesel engines, the amount of soot, or light blocking particles, is related to the concentration of DPM.

\subsubsection{Faraday Cup Electrometer (FCE)}

Perhaps the simplest type of electrical aerosol instrument, the Faraday cup electrometer consists of an electrometer and a filter inside of a Faraday cage. The principle of operation is the same as that for the PAS, except charging is done by a Faraday cup instead of a UV light. The FCE is useful in determining the concentrations of particles smaller that $10 \mathrm{~nm}$. It is used to determine nanoparticle concentrations that are undetectable with most PM instruments [18].

\subsubsection{Condensation Nucleus Counter (CNC)}

The condensation nucleus counter, also called the condensation particle counter (CPC), is a common instrument used to determine number concentrations of diesel

particulates [14]. Aerosol enters the $\mathrm{CNC}$ and is saturated with alcohol vapor. The mixture is cooled in condenser tubes and the vapor condenses on particles. This causes the particles to grow over $10 \mu \mathrm{m}$, making them suitable for optical detection. A beam of light is focused into a fine sample stream and re-focused on the other side into a 
photodetector. Pulses of light from individual particles are counted and interpreted. The CNC can be operated in one of two ways, counting mode or total intensity mode. The $\mathrm{CNC}$ is the key component in many different aerosol size distribution instruments. The $\mathrm{CNC}$ is capable of measuring particles between the sizes of $10 \mathrm{~nm}$ and $1000 \mathrm{~nm}$ [18]. There are many practical uses for the CNC including in-use HDDE testing, dynamometer HDDE testing, and ambient air testing. The European PMP program recommended that CNC measurements of non-volatile particles should be added to all regulatory PM measurement protocols [14].

\subsubsection{Cascade Impactor (Cl)}

An impactor is a device that is used to remove relatively large DPM particles from an exhaust stream. The stream is forced through an orifice and around an impaction plate. Particles that are large have excessive momentum and get thrown onto the impaction plate. The CI uses multi-stage impactors to classify particulates of decreasing size. Examples of CIs are micro-orifice uniform deposit impactors (MOUDI), nanomicro-orifice uniform deposit impactors (Nano-MOUDI), Andersen Mark3 Impactors, and low pressure impactors (LPI). CIs are capable of measuring particles as small as 10 nm. CIs have been available much longer than most particle sizing instruments. They were used in the 1990s to study composition and size distributions of DPM from direct injected diesel engines [19].

\subsubsection{Parallel Flow Diffusion Battery (PFDB)}

The parallel flow diffusion battery is an instrument capable of measuring particles in the size range of $3 \mathrm{~nm}$ to $56 \mathrm{~nm}$. With sponsorship from the United States EPA, and in collaboration with the University of Minnesota, the Particle Technology Laboratory 
(PTL), and the Lovelace Respiratory Research Institute (LRRI), the PFDB was developed during a Southwest Research Institute project. The PFDB consist of a scalper stage, a diffusion stage, and a CPC measurement stage. In the scalper stage, all particles above $56 \mathrm{~nm}$ are removed in order to prevent interference of larger particles during the other two stages. Inside the diffusion battery, there are three cells containing stacks of screens, and a fourth cell that contains small chambers. The screens rest in straight tubes, and they are designed to force particles within certain size ranges to diffuse and deposit onto their walls. The first cell contains screens that allow particles from $56 \mathrm{~nm}$ to $3 \mathrm{~nm}$ to pass. The screens in the second cell allow particles between $56 \mathrm{~nm}$ and $11 \mathrm{~nm}$ through. The third cell allows all particles between $56 \mathrm{~nm}$ and $19 \mathrm{~nm}$ particles to go through, and the fourth cell lets particles in the $56 \mathrm{~nm}$ to $35 \mathrm{~nm}$ size range to flow through. Each cell is connected to the sample inlet of the CPC, which allows for the number concentration measurement of the four size intervals: $3 \mathrm{~nm}$ to $11 \mathrm{~nm}, 11 \mathrm{~nm}$ to $19 \mathrm{~nm}, 19 \mathrm{~nm}$ to $35 \mathrm{~nm}$, and $35 \mathrm{~nm}$ to $56 \mathrm{~nm}$. Use of the PFDB is advantageous, because it can measure PM sizes that many instruments can not. [14].

\subsubsection{Electrical Low Pressure Impactor (ELPI)}

In an electrical low pressure impactor, aerosol particles are charged by a corona wire and forced through a multiple staged impactor. Unlike in a typical cascade impactor, each impactor plate is connected to an electrometer. When particles come in contact with a plate, an electrical current is induced and measured. Usually, an ELPI is able to measure particle concentrations and sizes at 12 stages. A near real-time measurement, at 2 seconds per scan, allows an ELPI to be used in transient testing. Post 
testing, particles can be analyzed gravimetrically because they are collected on the electrical impactor plates. A typical measuring range is $32 \mathrm{~nm}$ to $1000 \mathrm{~nm}$ [14].

\subsubsection{Scanning Mobility Particle Sizer (SMPS)}

The scanning mobility particle sizer is a DMA that operates under the same principle as a DMPS. The only difference is that the SMPS scans using an exponential ramp instead of a stepwise ramp. This decreases the total scan time from 20 minutes to 60 seconds. The SMPS is more commonly used than the DMPS because of the difference in scan time; but the reduction in scan time is accompanied by less accuracy. Since the DPM size distribution must remain constant during a scan, the SMPS is applicable where steady state conditions are reached. Diesel engines on heavy-duty machinery, boats, and trains operate at steady state most of the time. Also, the SMPS is frequently used as a standard in which to compare data from other instruments [14].

\subsection{RELATED STUDIES}

Findings from relevant research documents are presented below in order to supplement the implications made by this thesis. Some of the following equations in these related studies, such as the water adsorption equations, were used for the computations of this thesis.

\subsubsection{Particle Size Distribution from a Heavy-Duty Diesel Engine: Steady- State and Transient Emission Measurement Using Two Dilution Systems and Two Fuels}

Khalek et al. used a SMPS, an ELPI, and a PFDB to measure the particle size distribution and number concentration in the diluted exhaust of a heavy-duty diesel engine [21]. Two separate dilution systems were used with all three instruments to determine particle formation differences. In one system, a full flow CVS was combined 
with an ejector pump (CVS-EP). In the other, a double-ejector micro-dilution tunnel (DEMDT) was connected close to the turbocharger on the exhaust pipe. Two fuels, a low sulfur diesel (385 ppm) and an ultra low sulfur diesel (1 ppm), were examined over both steady state and transient engine operation. The selected engine was a $243 \mathrm{hp} 1999$ Navistar B250. Three identical hot-start FTPs were run, and five modes of the ISO 13 mode test were performed 3 times.

It was noted that all steady-state particle size distributions either fit a monomodal lognormal distribution or a bimodal lognormal distribution. In the nanoparticle size range, there was good agreement between the PFDB and the SMPS. The PFDB was complimentary to the ELPI in transient conditions, and it provided useful real time data. When the CVS-EP was used with the ultra low sulfur fuel, there was at least an order of magnitude reduction in nanoparticle emissions. With nearly no sulfur content, the $1 \mathrm{ppm}$ fuel still produced a detectable amount of nanoparticles, which points to significant nanoparticle contribution from volatile materials in the lube oil. Use of the CVS-EP resulted in more particle growth than when using the DEMDT. It was apparent that dilution conditions such as dilution ratio, residence time, and dilution temperature can have a dominant effect on nanoparticle size and concentration [21].

\subsubsection{Evaluation of Methods for Determining Continuous Particulate Matter from Transient Testing of Heavy-Duty Diesel Engines}

Jarrett and Clark worked on determining how to accurately compare integrated continuous TEOM data with conventional filter data from heavy-duty diesel engines. Part of their goal was to predict PM emissions in real-time. Water correction equations were used for the TEOM data, because moisture collection on the TEOM filter was responsible for about 20 percent, on average, of the mass reading. The use of two 
equations was necessary to determine the rate of water deposition on the filter. The water mass gain or loss on the filter for any given time period is given by the following equation:

$$
\mathrm{H}_{2} \dot{\mathrm{O}}_{\text {filter }}=\mathrm{C}_{1} *\left(\mathrm{C}_{2} * \mathrm{H}_{2} \mathrm{O}_{\text {air }}-\mathrm{H}_{2} \mathrm{O}_{\text {filter }}\right)
$$

\section{Equation 2}

The expression $\left(\mathrm{C}_{2} * \mathrm{H}_{2} \mathrm{O}_{\text {air }}-\mathrm{H}_{2} \mathrm{O}_{\text {filter }}\right)$ has units of $(\mathrm{g})$ and is the driving force of the water mass transfer. The term $\mathrm{H}_{2} \mathrm{O}_{\text {air }}$ has units of $(\mathrm{g})$ and is the moisture mass in the air that is flowing through the filter throughout a time period, $\Delta t$. The term $\mathrm{H}_{2} \mathrm{O}_{\text {filter }}$ has units of $(\mathrm{g})$ and is the moisture mass on the TEOM filter for the duration of time, $\Delta t$. Constant $C_{1}$ has units of $\left(\mathrm{s}^{-1}\right)$; it describes mass transfer and depends on flow rate through the filter. The constant $C_{2}$ is a unitless equilibrium constant for the ratio of moisture mass in the air to moisture mass on the TEOM filter. $C_{1}$ and $C_{2}$ were experimentally determined using steam injections at a specified mass rate. The moisture mass that passes through the TEOM filter can be given by the equation below.

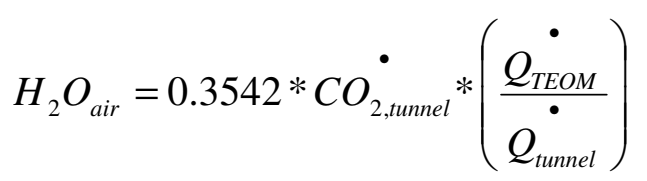

\section{Equation 3}

The term $Q_{\text {TEOM }}^{\bullet}$ is the overall volumetric flow rate through the TEOM, and $\dot{Q}_{\text {tunnel }}^{\bullet}$ is the volumetric flow rate through the dilution tunnel. The symbol $C O_{2, \text { tunnel }}^{\bullet}$ has units of $(\mathrm{g} / \mathrm{s})$ and is the $\mathrm{CO}_{2}$ mass flow rate through the dilution tunnel. 
It was clear that the TEOM collected significantly less mass than the conventional PM filters, so a best-fit correlation was determined as being

$$
P M_{\text {TEOM }}=0.8188 * P M_{\text {filter }}, \quad \text { Equation } 4
$$

where $P M_{\text {filter }}$ is the total PM mass collected over a complete cycle on a conventional filter (g/cycle), and $P M_{\text {TEOM }}$ is the total amount of PM mass as indicated by the TEOM.

It was concluded that instantaneous TEOM data may be unreliable due to the transient moisture mass on the filter, but a water correction approach can be taken in order to provide a closer relationship between TEOM mass data integrated over a cycle and conventional filter-based PM mass. It was also found that continuous CO data can be used to approximate PM mass on a filter throughout a cycle, but large errors may occur on an instantaneous basis [22].

\subsubsection{A Fast Response Particulate Spectrometer for Combustion Aerosols}

In 2002, Reavell et al. detailed the functionality of a new fast response particulate spectrometer called the DMS-500 [23]. They noted that, because other PM sources only become dominant far away from traffic, the greatest contributor to fine particulate matter in the urban environment is road transport. Since traffic sources generate the majority of respired particles (which mostly show up in modes falling in the range of $5 \mathrm{~nm}$ to 1000 $\mathrm{nm})$, it was important for an instrument to be capable of real-time PM concentration measurement for all particle sizes between $5 \mathrm{~nm}$ and $1000 \mathrm{~nm}$. The DMS was designed specifically to measure such particles. Initial calibration was accomplished using a vane pump and mists of oil. After the electrometer current/particle density coefficients were determined and modeled, the system was validated by comparing the DMS aerosol response to a spectrum of aerosol responses from an SMPS. Light Duty Diesel Engine 
(LDDE) test were run in which the DMS and SMPS sampled directly from a standard dilution tunnel. This limited the total response time to about $500 \mathrm{~ms}$. The final result was a good general agreement between the DMS and the SMPS, where the DMS had extra bandwidth and was able to show much greater detail than the SMPS. The central conclusion was that a new instrument was available that could measure the fine particle number spectrum with an unmatched frequency response. The DMS size classification resulted in clear resolution of accumulation and nucleation modes. Even detecting the transient effects on particulate matter from engine events such as gear changes and fuel cuts was possible [23].

\subsubsection{Effective Density of Diesel Exhaust Particles as a Function of Size}

Lappi et al. developed a method to define effective particle density as a function of diesel particle size. The method was to measure both the aerodynamic and mobility size distributions of sample aerosol, obtain an agreement between size dependent effective density, and obtain a best-fit line between the distributions. Aerodynamic size distribution was measured with an ELPI, and mobility size distribution was measured with a SMPS. It was realized that a heated ejector diluter helps prevent nucleation and condensation, and it is to be used when the structure of agglomerated particles is being studied. The density of primary particles, or particles smaller than $30 \mathrm{~nm}$, was typically constant at about $1.1 \mathrm{~g} / \mathrm{cc}$, and the particles between $20 \mathrm{~nm}$ and $80 \mathrm{~nm}$ appeared to be almost spherical. As particle size increased after $30 \mathrm{~nm}$, the effective density decreased almost linearly (due to void space) from $1.1 \mathrm{~g} / \mathrm{cc}$ to about $0.2 \mathrm{~g} / \mathrm{cc}$. Various fuels were used in the study, which led to the conclusion that dilution has a stronger relationship with effective particle density than does fuel type [24]. 


\subsubsection{Evaluation of Methods for the Determination of Diesel-Generated Fine Particulate Matter: Physical Characterization Results}

Kinsey et al. conducted a study at the WVU EERC with two diesel engines and a dynamometer to compare common PM measurement techniques. Secondary dilution was done with a $20.3 \mathrm{~cm}$ stainless steel tunnel with TSI Model 3708 flow splitters connected to each sampling probe. The experiment was a multi-phase instrument comparison to evaluate inter- and intra-method variability. Included in the evaluation were a TEOM, three SMPSs, a CPC, a TSI DustTrak, a MET-One E-BAM, two ELPIs, and two timeintegrated filter samplers. The SMPSs and the ELPIs generally provided the most comparable results for number distribution, but the TEOM proved to have the most accurate correlation with the gravimetric filter method. SMPS and ELPI measurements could not be compared to the CPC due to problems with the coupled TSIs capillary diluters. It was recommended that the TSI/CPC diluter combination should not be used for future research [25].

\subsubsection{Research on Diesel Exhaust and Other Particulates}

Health Effects Institute (HEI) is committed to understanding human health effects due to exposure to diesel exhaust. As of 2003, HEI had taken part in more than forty research projects and published three special reports (HEI Diesel Epidemiology Working Group 2002; HEI Diesel Epidemiology Expert Panel 1999; and HEI Diesel Working Group 1995) that evaluated the scientific literature concerning diesel health effects. Epidemiologic studies of people who were occupationally exposed (truck drivers and railroad workers) show a weak but fairly consistent association between exposure to DPM and lung cancer [7]. Long-term rat, mice, and hamster bioassays showed an increase in lung tumors only in rats with high levels of exposure (greater than three 
milligrams of DPM per cubic meter). It was likely that the carcinogenesis in rats was related to lung overload of particles rather than the absorption of organic compounds and gases. Still, the possibility of other cancer causing mechanisms at lower levels of exposure is possible. The EPA and the California EPA both concluded that the results of animal studies infer human hazard, but there is too much uncertainly for the information to be used in QRA for human lung cancer. Human exposure to low ambient concentrations may not even be relevant to the extremely high-dose experiments in animal studies. Exposure of high concentrations of fresh diesel aerosol $\left(300 \mathrm{ug} / \mathrm{m}^{3}\right.$ for 1 hour) to human participants took place in order to evaluate the effects of PM on human airways. Changes in some inflammatory markers, such as neutrophils and interleukin in sputum, neutrophils and platelets in blood, and bronchial inflammatory cells were exhibited, but there was no change in lung function. Both healthy participants and asthmatic participants took part in the study, and a central conclusion was made that healthy individuals may have different responses to DPM than asthmatic individuals. It was previously suggested that diesel exhaust exposure increases specific allergic responses to their respective allergens, but no studies have shown such [7].

\subsubsection{Particles from Internal Combustion Engines - What We Need to Know}

In 2000, Collings and Graskow conducted an experiment where they measured particle size distributions with an SMPS under dilution tunnel conditions that mimicked atmospheric dilution conditions [26]. A sample was taken from a $2.5 \mathrm{~L}$ diesel engine's exhaust, kept warm in a short insulated sample line, and diluted in an elementary dilution tunnel before being measured by the SMPS. The use of fuels of various sulfur contents showed that fuels that are lower in sulfur yield fewer nanoparticles. The researchers 
ultimately suggested that ample knowledge of the relationship between dilution and particle formation could precipitate insight that would allow particle emissions to be reduced by active on-vehicle manipulation of the atmospheric dilution process [26].

\subsubsection{Evaluation and Correction of Moisture Adsorption and Desorption from a Tapered Element Oscillating Microbalance}

Jarrett et al. explain how the TEOM shows promise in determining real-time continuous PM, but moisture biases TEOM data through its adsorption and desorption from the TEOM's oscillating filter [27]. The purpose of their study was to model the moisture effect on the TEOM for transient tests, compensate for the biased data using experimentally determined coefficients and other continuous data, and present a much more accurate trace of PM. Results showed that ambient moisture in the air had a strong linear correlation with moisture mass on the TEOM filter. Injecting steam into the engine's exhaust pipe was a method that was used to evaluate the effects of moisture on the filter. Possible bias due to volatile $\mathrm{HC}$ from combustion was assumed to be negligible, because of its mass rate, on average, being about a thousandth of the moisture mass rate through the filter. Essentially, five equations are needed to correct for water loading. The chief equation that accounts for water adsorption/desorption rate is

$$
\text { TEOM }=\dot{P M}_{\text {filter }}+\mathrm{H}_{2} \dot{\mathrm{O}}_{\text {filter }}, \quad \text { Equation } 5
$$

where the total mass rate given by the TEOM is TEOM, the legitimate PM mass rate on the filter is given by $P \dot{M}_{\text {filter }}$, and the moisture mass rate on the filter is given by $\mathrm{H}_{2} \dot{\mathrm{O}}_{\text {filter }}$. Also, $\mathrm{H}_{2} \dot{\mathrm{O}}_{\text {filter }}$ can be expressed using the equation

$$
\mathrm{H}_{2} \dot{O}_{\text {filter }}=\frac{\Delta \mathrm{H}_{2} \mathrm{O}_{\text {filter }}}{\Delta t}, \quad \text { Equation } 6
$$


in which $\Delta \mathrm{H}_{2} \mathrm{O}_{\text {filter }}$ is the change in water mass on the filter over the time span, $\Delta t$. Since the ambient moisture is related to the moisture mass on the filter, $\mathrm{H}_{2} \dot{\mathrm{O}}_{\text {filter }}$ can also be given by

$$
\mathrm{H}_{2} \dot{\mathrm{O}}_{\text {filter }}=\mathrm{C}_{1} *\left(\mathrm{C}_{2} * \mathrm{H}_{2} \mathrm{O}_{\text {air }}-\mathrm{H}_{2} \mathrm{O}_{\text {filter }}\right), \quad \text { (same as Equation 2) }
$$

where $\mathrm{H}_{2} \mathrm{O}_{\text {air }}$ is the moisture mass in the air that passes through the filter over a time period, $\Delta t$, and $\mathrm{H}_{2} \mathrm{O}_{\text {filter }}$ is the moisture mass in the filter over the same time period, $\Delta t$. $C_{1}$ and $C_{2}$ are experimentally determined coefficients that depend on many factors, but their values are thought to be mainly attributed to the chemical attraction between water molecules and the TEOM filter. $C_{1}$ and $C_{2}$ were roughly determined from 11 different transient tests, resulting in an average $C_{1}$ of $0.0723(51.25 \% \mathrm{COV})$ and an average $C_{2}$ of $0.0119(75.61 \% \mathrm{COV})$. The moisture mass on the filter can be calculated from the humidity ratio for the filter, $\omega_{\text {filter }}$, the filter moisture mass, $\mathbf{M}_{\mathrm{H}_{2} \mathrm{O}}$, and the net filter mass, $\mathbf{M}_{\text {filter }}$, in the equation

$$
\omega_{\text {filter }}=\frac{\mathrm{H}_{2} \mathrm{O}_{\text {filter }} *\left(\mathrm{M}_{\mathrm{H}_{2} \mathrm{O}}-\mathrm{M}_{\text {filter }}\right)}{\mathrm{M}_{\text {filter }}{ }^{2}} .
$$

\section{Equation 7}

The moisture mass of the air that passes through the filter can be determined from the equation

$$
\omega_{\text {air }}=\frac{H_{2} O_{a i r}}{\mathrm{M}_{\text {air }}}=0.622 *\left(\frac{\phi^{*} p_{g}}{p-\phi^{*} p_{g}}\right)
$$

\section{Equation 8}

where $\omega_{\text {air }}$ is the humidity ratio for air, $\mathbf{M}_{\text {air }}$ is the mass of dry air for a given volume, 0.622 is the ratio for the molecular weight of dry air to water, $\phi$ is the relative humidity as a fraction, $p_{g}$ is water's saturation vapor pressure at $70^{\circ} \mathrm{F}$, and $p$ is the barometric 
pressure. It was pointed out that the inclusion of a term for PM accumulation would provide a more accurate and more advanced model for the change in moisture mass on the TEOM. The results showed that the model that was developed yielded a representation of instantaneous PM that was much improved in accuracy. [27]

\subsubsection{Significance of Fuel Sulfur Content and Dilution Conditions on Particle Emissions from a Heavily-Used Diesel Engine During Transient Operation}

In a 2007 SAE paper by Kittelson et al., the effects of fuel sulfur content and dilution ratio on PM concentrations were discussed. The research was done using a 1992 International 7.3 L diesel engine, two ejector type micro-diluters, an EEPS, a SMPS, and a CFR Subpart N compliant CVS with critical flow orifices. The dilution ratios of the sequential ejector diluters were documented to be 30 and 6.17 , which provided a total secondary dilution ratio of 185 . The primary dilution ratio ranged from 4.2 to 30.1 , depending on operating conditions. For each fuel (309 ppm sulfur and 9.2 ppm sulfur), the particle concentrations were measured over an acceleration during an FTP, a deceleration during an FTP, and steady state operations of ISO-8178 modes 1, 8, and 11. The primary tunnel flow was set to $1500 \mathrm{scfm}\left(0.71 \mathrm{~m}^{3} / \mathrm{s}\right)$ and $2750 \mathrm{scfm}\left(1.3 \mathrm{~m}^{3} / \mathrm{s}\right)$, which resulted in "low" and "high" dilution ratios, respectively, for each testing circumstance. A conclusion of interest was that "the nuclei mode [was] significantly larger in concentration for the $1.3 \mathrm{~m}^{3} / \mathrm{s}$ tunnel." The reason described for this was that "the higher dilution ratio provides the necessary cooling for optimal nucleation." For the entirety of the study, the conclusion that nuclei mode particles, specifically the ones smaller than $20 \mathrm{~nm}$, were solitarily affected significantly by dilution ratio was the only one made that related dilution ratio to particle concentrations. The other key conclusion, which agreed with the conclusions of Khalek et al. in "Particle Size Distribution from a 
Heavy-Duty Diesel Engine: Steady-State and Transient Emission Measurement Using

Two Dilution Systems and Two Fuels," was that the formation of a nuclei mode can be reduced by using diesel fuels of lower sulfur content [28].

\subsection{PM REDUCTION TECHNOLOGIES}

For diesel engines, there are several means to decrease PM emissions. As discussed above in the Related Studies section, it has been shown that sulfur content in diesel fuel affects particle formation. Fuels that are low in sulfur tend to generate fewer nanoparticles. Note that fuel properties, such as cetane number, greatly affect PM emissions. Mostly due to their oxygenated structure, the use of neat biodiesel fuels over petroleum diesel fuels generally leads to about a $75 \%$ decrease in brake-specific PM emissions. The engine control unit (ECU), which directly controls injection timing, can be manipulated for a wide variety of operating conditions in order to minimize PM emissions. However, there is a $\mathrm{NO}_{\mathrm{x}}-\mathrm{PM}$ tradeoff. When an engine is being controlled to reduce PM, there is an increase in $\mathrm{NO}_{\mathrm{x}}$ emissions. Perhaps the most useful device to date for the reduction of PM emissions is the diesel particulate filter (DPF). There are different types of DPFs, none of which provide the same pressure drop, filtration efficiency, durability, cost, and production suitability. Paper filters lack the ability to be regenerated and are generally used for short-lived indoor applications. Metal fiber flowthrough filters have cores that are made from woven metal fibers, and they can be repeatedly regenerated by passing electric currents through and heating the internal fibers. Silicon carbide wall-flow filters consist of a single piece or segmented SiC core, and they have a high melting temperature $\left(2700{ }^{\circ} \mathrm{C}\right)$ which allows them to be regenerated through exhaust temperature increase by engine control. Cordierite wall-flow filters are 
similar in geometric structure to silicon carbide filters. They can be used in the same applications and are regenerated in the same fashion; but they have a much lower melting temperature $\left(1200{ }^{\circ} \mathrm{C}\right)$ which is responsible for their occasional melt down during the regeneration process. DPFs have PM trapping efficiencies (mass based) of between $50 \%$ and $99 \%$. A simple and effective way for PM emissions to be reduced is fuel conservancy. With any diesel engine technology, the magnitude of pollution from combustion is proportional to the amount of fuel used. 


\section{EXPERIMENTAL SETUP AND PROCEDURES}

\subsection{OVERTURE}

The data from this study was taken from a full-scale critical flow venturi (CFV) dilution tunnel at the WVU Center for Alternative Fuels, Engines, and Emissions (CAFEE). The laboratory is compliant with the standards set by ISO 8178 and 40 CFR Part 86, Subpart N [29]. Multiple engines were used in this study in order to ensure that findings hold for the majority of engine technologies. The test cell was designed for regulated emissions testing, and it contains four essential components. A dynamometer was used to measure and control engine parameters such as speed and torque during test cycles. A CFV dilution tunnel provided an environment that allowed for consistent DPM formation and measurement. Overall fuel consumption was measured with a digital fuel scale; and a computer-based data acquisition system (DAQ) was used to record the laboratory signals. Additional emissions sampling equipment included a constant flow ejector diluter, a secondary $\mathrm{NO}_{\mathrm{x}}$ analyzer, and a DMS-500 fast particle sizer. The primary laboratory apparatus is illustrated in Figure 11. The additional components are within the perimeter of the dashed line, and they have an experimental arrangement that is unique to this work. 


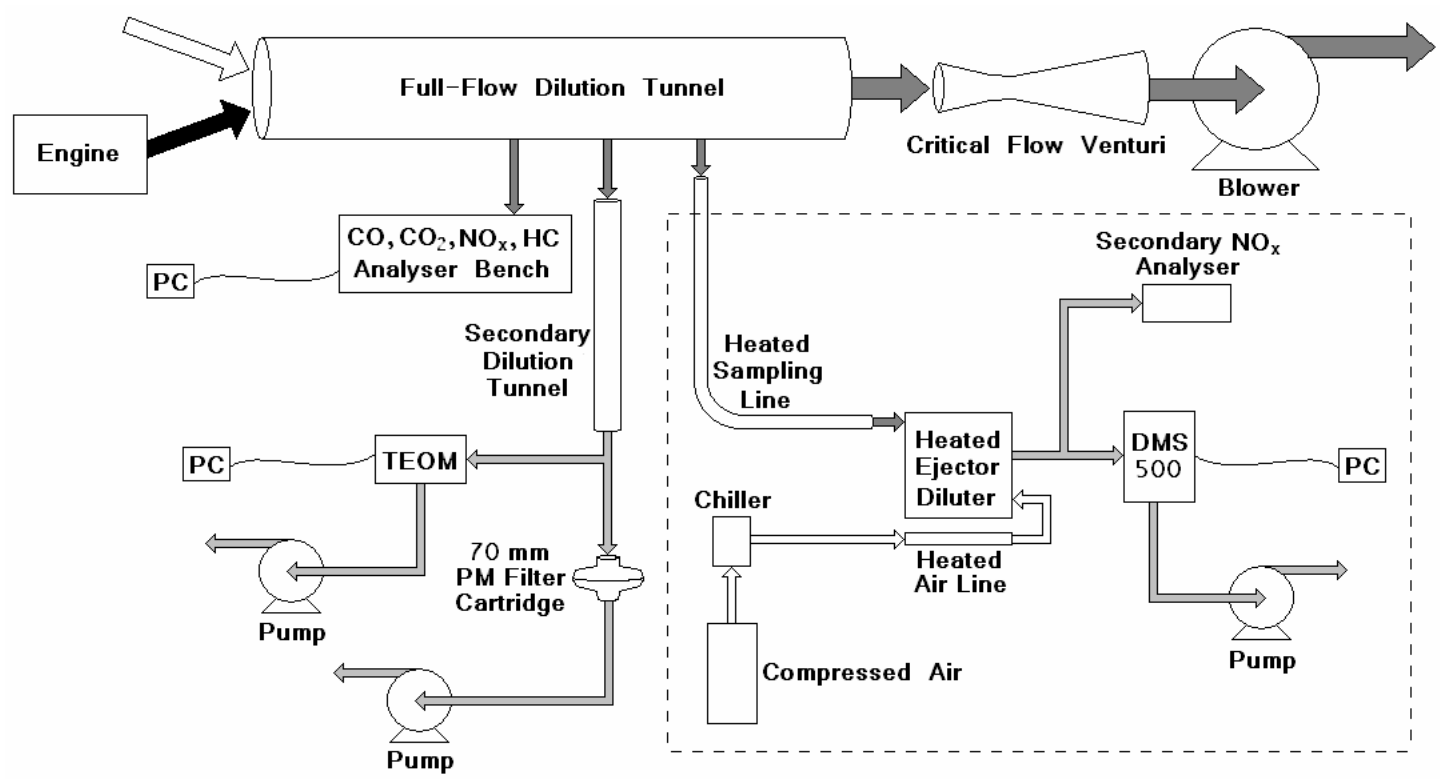

Figure 11 Components of Exhaust Sampling System

\subsection{TEST CYCLES}

Test Cycles are used for emissions certification and fuel economy determination, and they are designed for the dynamometer/engine control of both chassis dynamometer systems and engine dynamometer systems. Light-Duty Diesel Engines (LDDE) include truck classes 1 through 3 , and power trucks with a gross vehicle weight rating (GVWR) of up to 14,000 lb. Heavy-Duty Diesel Engines (HDDE) consist of truck classes 6 through 8 , and power trucks with a GVWR of 19,500 $\mathrm{lb}$ and up. For engine certification within the US, LDDEs are tested on chassis dynamometers, and HDDEs are tested on engine dynamometers.

There are many test cycles designed for HDDE dynamometer testing. Some simulate what engines experience during on-road operation, while others are designed to test engines at steady-state conditions. The Federal Test Procedure (FTP), and the Supplemental Emissions Test (SET) are two engine dynamometer test cycles that are used in the U.S. for on-road HDDE certification. Engine manufacturers are required to 
perform both dynamometer and in-use emissions testing before any particular on-road HDDE can be sold in the U.S. The European Union uses the ETC (FIGE transient) and the ECE R49 for engine certification. The ETC is a transient cycle for truck and bus engines, and the ECE R49 is a 13 mode steady-state cycle. In Japan, the government uses the JE05, which is a transient cycle used for the certification of HDDEs and gasoline engines.

\subsubsection{Federal Test Procedure}

The FTP was designed to simulate speeds and loads that an engine would experience on the road. For heavy-duty diesel engines, the FTP simulates stop and go city driving and freeway driving for a bus or truck. To simulate driving conditions as accurately as possible, the FTP includes segments of the test where the dynamometer motors the engine. The FTP is composed of four separate sections that, in the following order, represent the New York non-freeway (NYNF), the Los Angeles non-freeway (LANF), the Los Angeles freeway (LAFY), and a repeat of the NYNF. The NYNF segment simulates the frequent starts and stops of light urban traffic. The LANF section simulates slow-moving heavy urban traffic with few stops, and the LAFY section simulates busy freeway traffic [29]. Figure 12 is an engine speed versus time plot for an actual FTP cycle. Below Figure 12, Figure 13 is an engine torque versus time plot for the same test. 


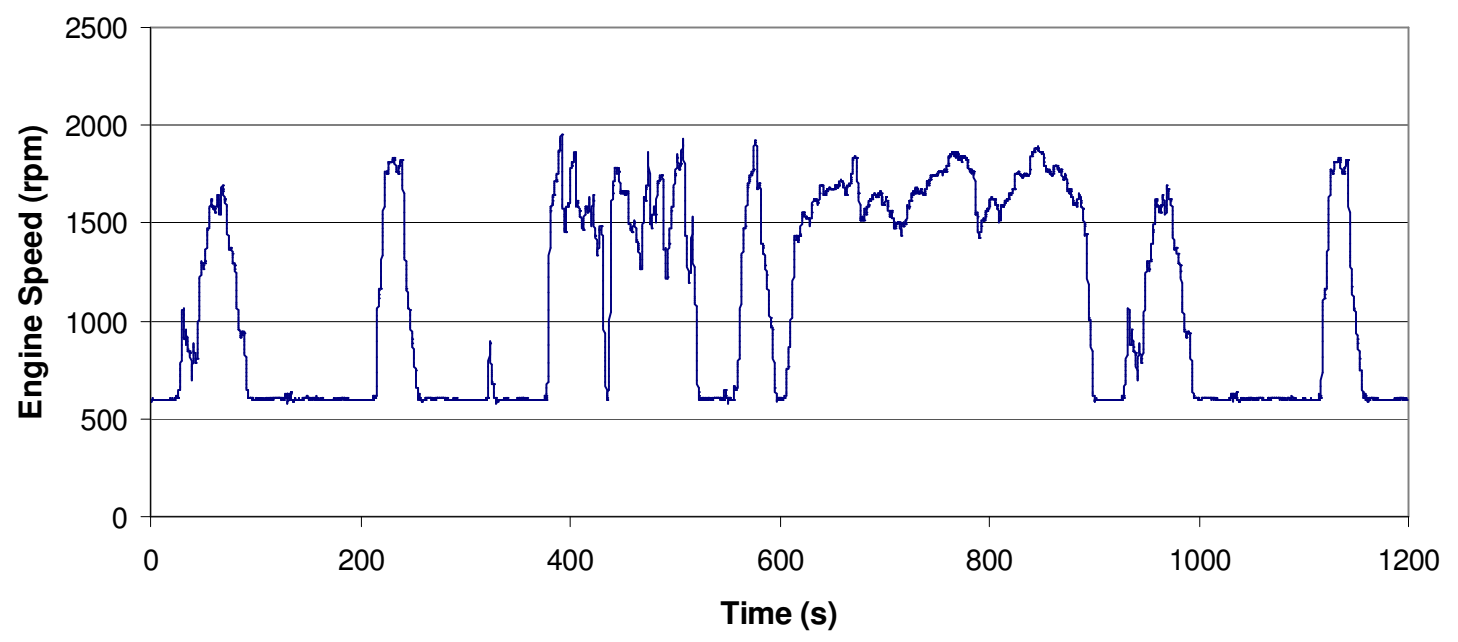

Figure 12 Engine Speed vs. Time for a FTP Cycle

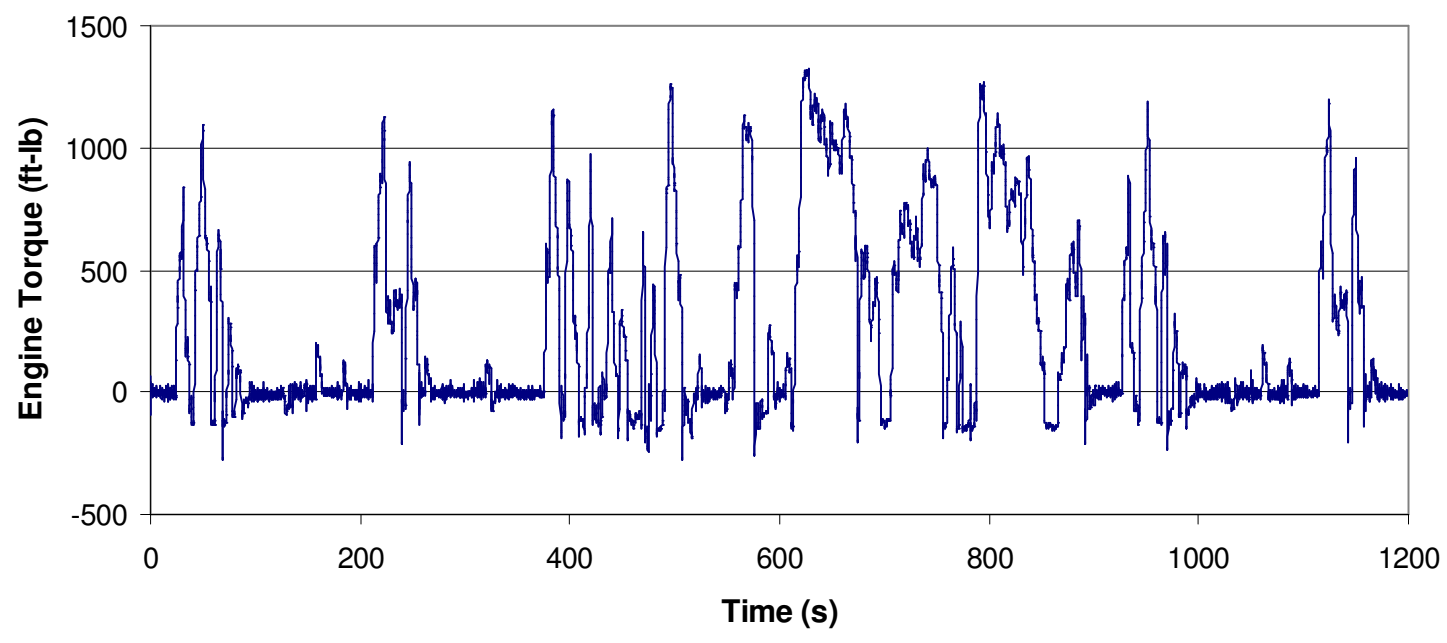

Figure 13 Engine Torque vs. Time for a FTP Cycle

The plots in Figures 12 and 13 were generated during an actual dynamometer test by a 1992 DDC S60 Engine. The target speed and torque points were created in accordance with a preliminary engine map. 


\subsubsection{Supplemental Emissions Test}

Also called the ESC or the OICA/ACEA, the SET is used for HDDE testing in the U.S. to supplement the FTP. It has 13 steady-state modes that cover a matrix of engine speeds and loads. Table 4 is an example of engine set points for the SET.

Table 4 Example SET Cycle

\begin{tabular}{|c|c|c|c|c|c|c|}
\hline Mode & $\begin{array}{c}\text { Engine Speed } \\
(\mathrm{rpm})\end{array}$ & $\begin{array}{c}\text { Load } \\
(\%)\end{array}$ & $\begin{array}{c}\text { Set Torque } \\
(\mathrm{ft}-\mathrm{lb})\end{array}$ & $\begin{array}{c}\text { Power } \\
(\mathrm{hp})\end{array}$ & $\begin{array}{c}\text { Weight } \\
\text { Factor (\%) }\end{array}$ & $\begin{array}{c}\text { Duration } \\
(\mathrm{min})\end{array}$ \\
\hline 1 & 600 & 0 & 0 & 0 & 15 & 4 \\
\hline 2 & 1199 & 100 & 1418 & 324 & 8 & 2 \\
\hline 3 & 1422 & 50 & 668 & 181 & 10 & 2 \\
\hline 4 & 1422 & 75 & 1002 & 271 & 10 & 2 \\
\hline 5 & 1199 & 50 & 709 & 162 & 5 & 2 \\
\hline 6 & 1199 & 75 & 1064 & 243 & 5 & 2 \\
\hline 7 & 1199 & 25 & 355 & 81 & 5 & 2 \\
\hline 8 & 1422 & 100 & 1336 & 362 & 9 & 2 \\
\hline 9 & 1422 & 25 & 334 & 90 & 10 & 2 \\
\hline 10 & 1644 & 100 & 1179 & 369 & 8 & 2 \\
\hline 11 & 1644 & 25 & 295 & 92 & 5 & 2 \\
\hline 12 & 1644 & 75 & 884 & 277 & 5 & 2 \\
\hline 13 & 1644 & 50 & 590 & 185 & 5 & 2 \\
\hline
\end{tabular}

Regulated emissions measurements are taken and a final value for each one is found using averaging and weighting factors for each mode. A SET is valid if the engine speed is held to within $\pm 50 \mathrm{rpm}$ and the torque is held to within $\pm 2 \%$ of the maximum torque value. 


\subsection{TEST ENGINES}

The engines tested were all on-road heavy-duty diesel engines. Several modifications were made in order to adapt the engines for a laboratory environment. Radiators were replaced with a water/coolant heat exchanger, and all accessories, such as fans and air-conditioning units, were removed. Mufflers were replaced by an exhaust backpressure valve, and the CAFEE heating, ventilating, and air conditioning (HVAC) system supplied the intake air. Table 5 displays basic specifications for the engines used in this study.

Table 5 Test Engine Specifications

\begin{tabular}{|c|c|c|c|}
\hline Engine Manufacturer & Detroit Diesel Corporation & Detroit Diesel Corporation & Cummins \\
\hline Engine Model, Year & DDC Series 60, 1992 & Rebuilt DDC Series 60, 1992 & ISM 370, 2004 \\
\hline Configuration & Inline 6 cylinder & Inline 6 cylinder & Inline 6 cylinder \\
\hline Displacement (L) & 12.7 & 12.7 & 10.7 \\
\hline Power Rating (hp) & $360 @ 1810 \mathrm{rpm}$ & $360 @ 1810 \mathrm{rpm}$ & $370 @ 2100 \mathrm{rpm}$ \\
\hline Torque Rating (ft-lb) & $1450 @ 1200 \mathrm{rpm}$ & $1450 @ 1200 \mathrm{rpm}$ & $1450 @ 1200 \mathrm{rpm}$ \\
\hline Compression Ratio & $15: 1$ & $15: 1$ & $16.5: 1$ \\
\hline Bore $\times$ Stroke (mm x mm) & $135 \times 165$ & $135 \times 165$ & $125 \times 147$ \\
\hline Air Handling & Turbocharded, Aftercooled & Turbocharded, Aftercooled & VGT, Aftercooled \\
\hline Exhaust Gas Recirculation (EGR) & N/A & N/A & Cooled EGR \\
\hline
\end{tabular}

The engines in the table above generate about the same amount of power. It was chosen to use three engines with similar specifications, so that it would be more apparent how changes in engine technology from 1992 to 2004 effect DPM formation. 


\subsubsection{Detroit Diesel Series 60}

The 1992 DDC was acquired by WVU for use in laboratory emissions testing, and it has no available history prior to procurement. An in-use picture of the engine and a speed/torque map are shown below in Figures 14 and 15.

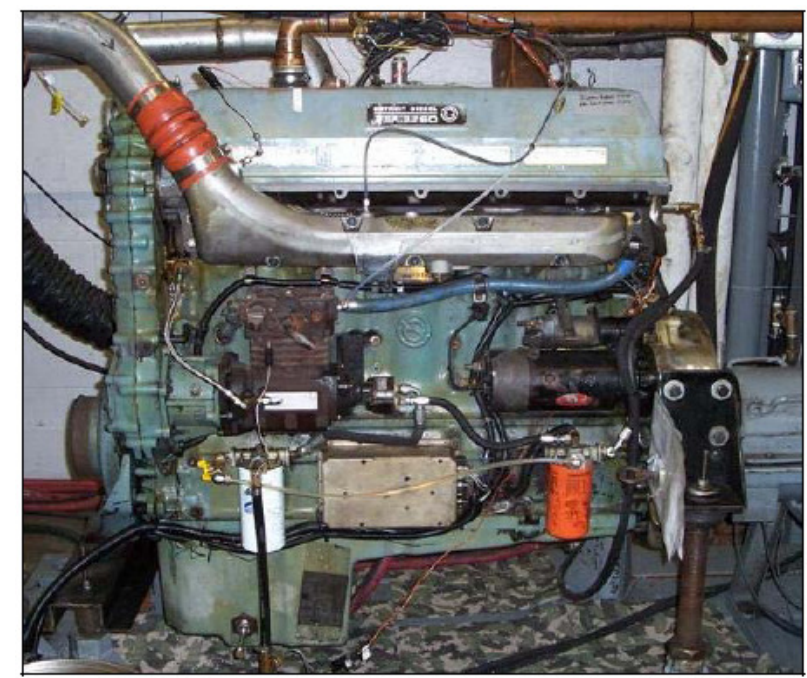

Figure 141992 DDC Series 60 [29]

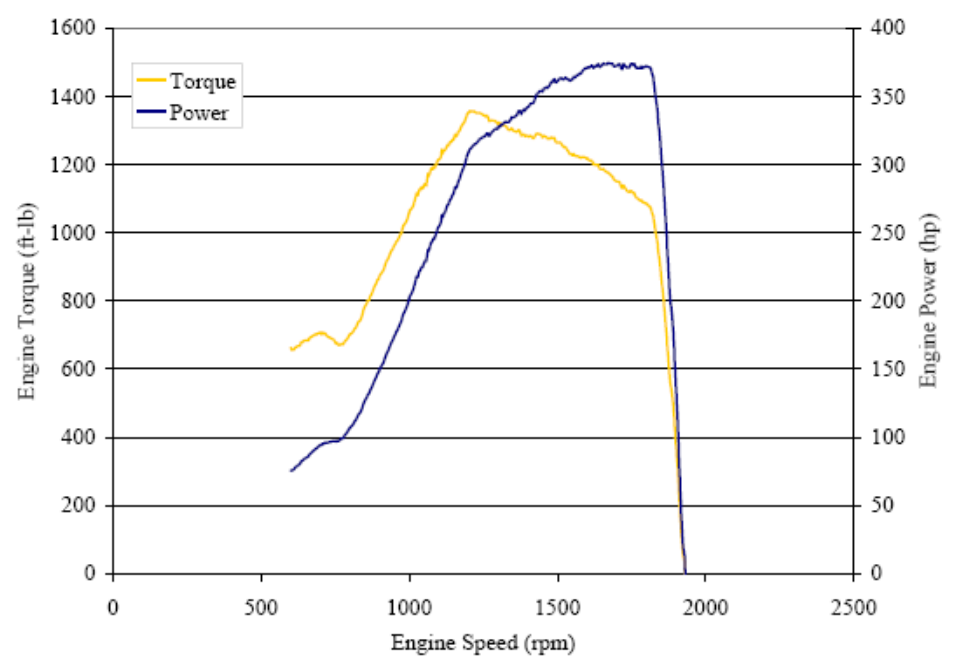

Figure 151992 DDC Series 60 Engine Map [29] 


\subsubsection{Rebuilt 1992 Detroit Diesel Series 60}

The Rebuilt 1992 DDC was taken from a salvage yard by WVU and rebuilt in 2006. Like the other 1992 DDC S60, this engine came with no history of use or maintenance. It was entirely rebuilt to meet its original performance and emissions specifications, and it was aged for 100 hours on the dynamometer before it was used for emissions testing. Figure 16 shows the engine below, and Figure 17 is an experimentally determined performance map used to calculate FTP speed and torque set points.

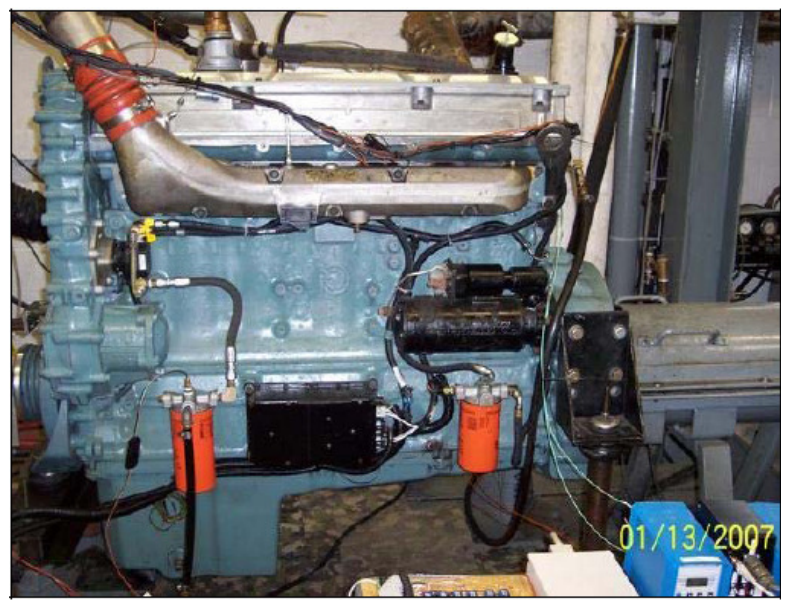

Figure 16 Rebuilt 1992 DDC Series 60 [29]

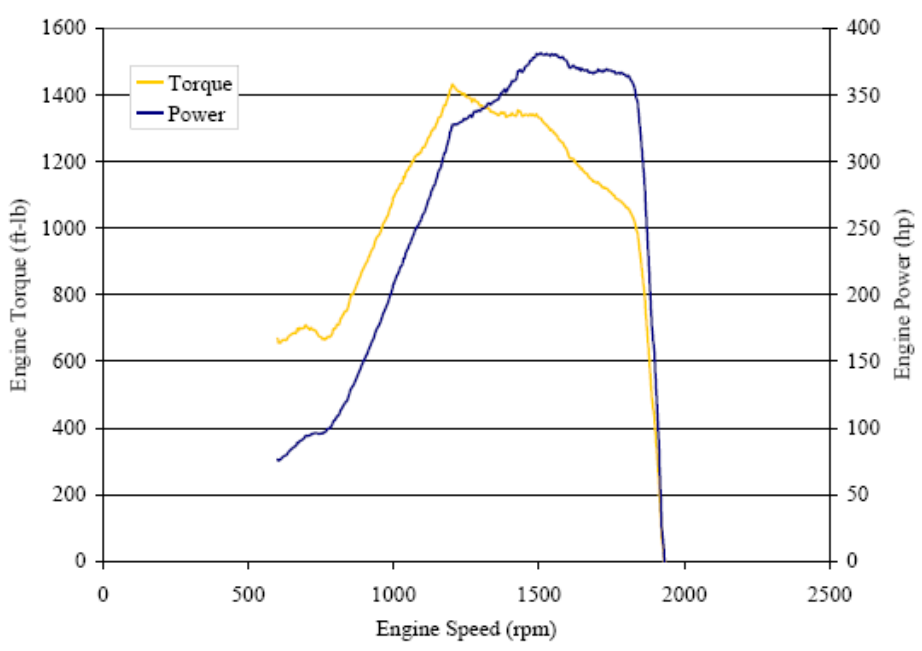

Figure 17 Rebuilt 1992 DDC Series 60 Engine Map [29] 


\subsubsection{Cummins ISM 370}

The 2004 Cummins was procured directly from Cummins. It differs significantly from the two DDCs in that it incorporates more advanced technologies. It uses cooled exhaust gas recirculation (EGR) and a variable geometry turbocharger (VGT) to minimize $\mathrm{NO}_{\mathrm{x}}$ emissions while maintaining performance and fuel economy. The 2004 Cummins can be seen in Figure 18, and one of its maps is shown in Figure 19.

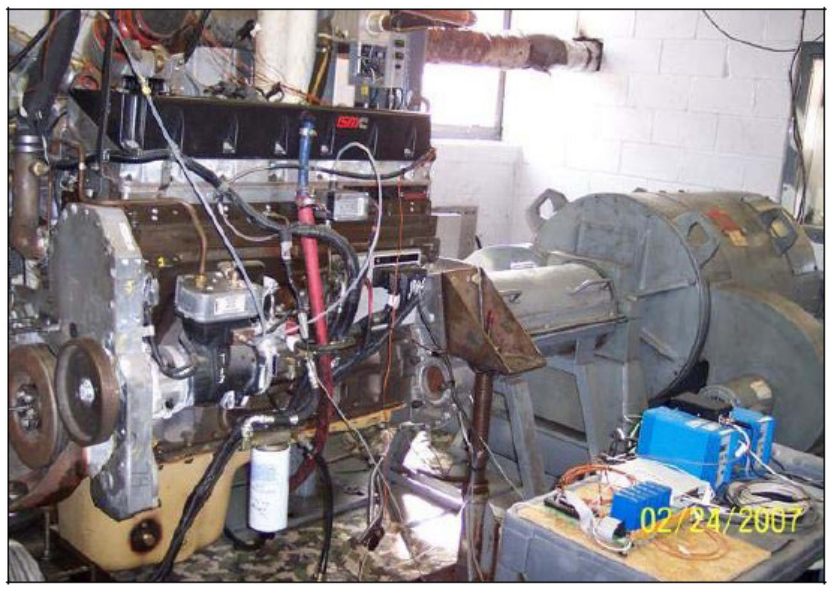

Figure 182004 Cummins ISM 370 [29]

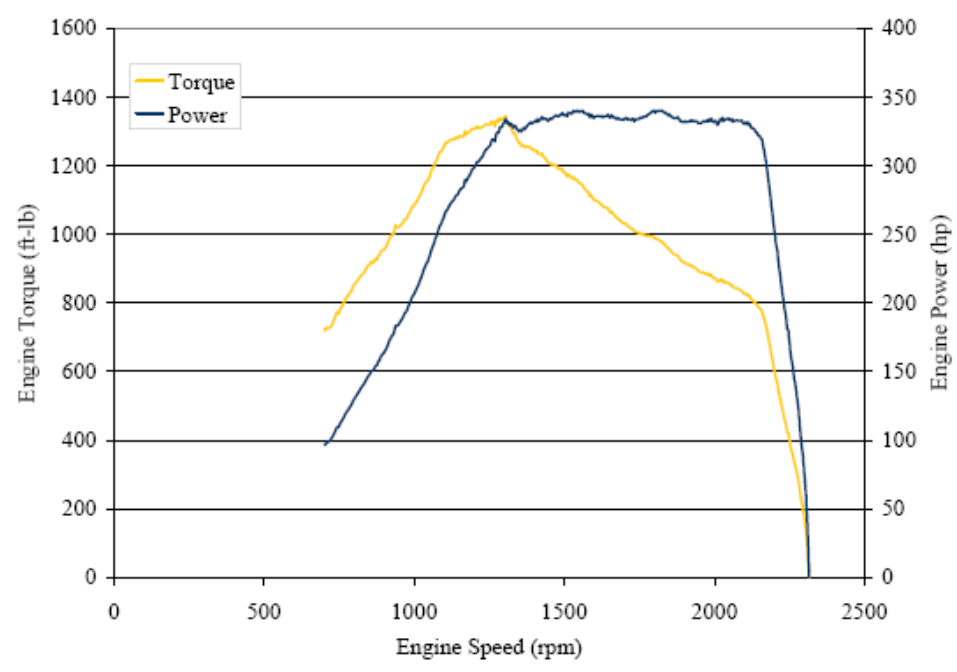

Figure 192004 Cummins ISM 370 Engine Map [29] 


\subsection{TEST FUELS}

Apart from the supplemental emissions tests that were run to determine the effects of relative humidity or dilution ratio on particle formation, all test cycles were run for purposes other than particle sizing research. Since sampling of aerosol with the DMS was opportunistic rather than scheduled, the assortment of fuels that was used in this research was not specifically selected for the achievement of the initial goals. If additional laboratory time and resources were available, a more strategic selection of fuels would have been implemented.

Table A-1 in the Appendix provides a summary of the fuels used and their respective tests. Many of the fuels were being tested for certification purposes; and due to proprietary issues, it was not possible to include their fuel property information. Tables 9 and 10, in the Base Fuels vs. Additized Fuels section of this thesis, display full fuel property analyses of the low cetane fuels used in a comparison study between a base fuel and nine versions of the same fuel with additives.

\subsection{LABORATORY COMPONENTS}

\subsubsection{Dynamometer}

The dynamometer was always operated and calibrated in accordance to 40 CFR, Part 86, Subpart N [29]. The DYC 243 dynamometer implemented in this study is a direct current model made by General Electric. It is a computer-commanded dynamometer, and it controlled the engine speed and torque by applying and absorbing rotational forces that an in-use drive shaft would present. A proportional-integralderivative (PID) throttle positioning program was used to specify and tune the engine's throttle position in order to supply positive power and meet the FTP regression 
requirements. The dynamometer produced an engine speed signal from an attached digital encoder. Engine torque was determined analytically from force measurements taken by the dynamometer's load cells. The dynamometer was joined to the engine with a Vulkan coupling and drive shaft. It was capable of providing up to $500 \mathrm{hp}$ and absorbing as much as $550 \mathrm{hp}$. Figure 20 shows the dynamometer as it was when testing took place.

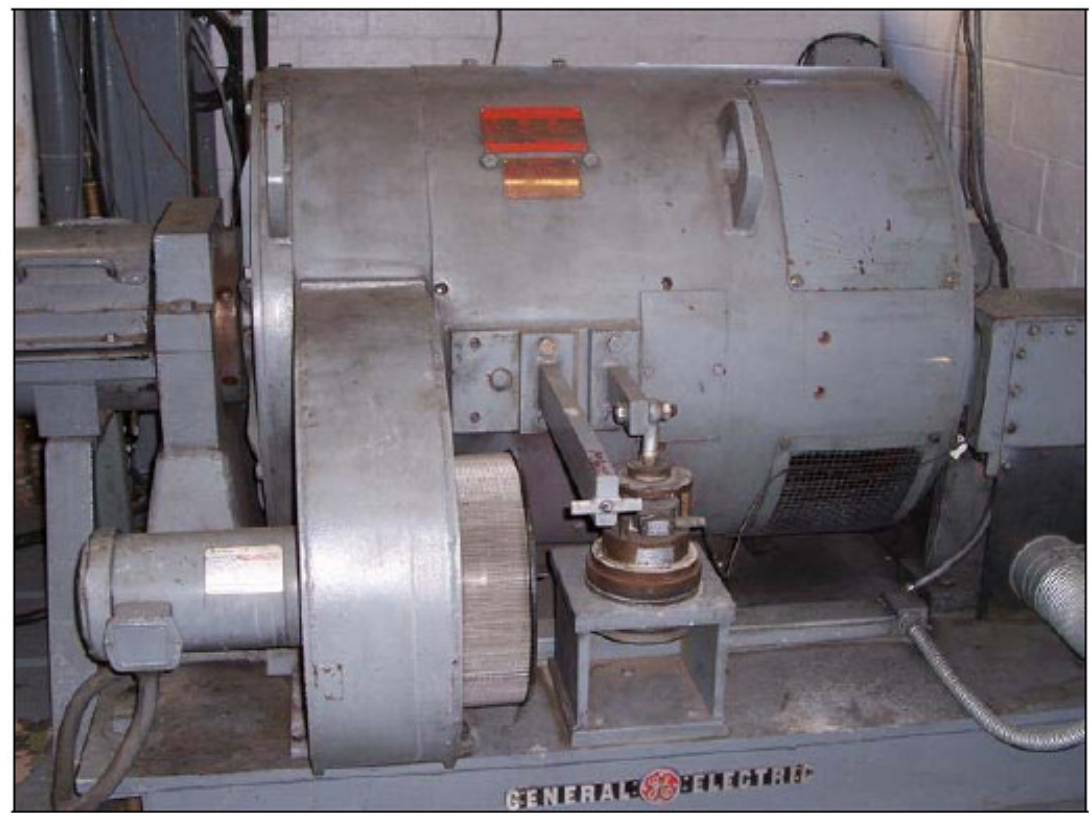

Figure 20 DYC 243 Dynamometer

\subsubsection{Engine Control and Data Acquisition System}

All of the measurements that were continuously taken during testing were converted into digital signals by the DAQ's "3B" modules and recorded in a laboratory file. Some of these continuous measurements were necessary for the computer to accurately control the engine, while other measurements, such as the gaseous emissions signals and intake air humidity, were taken for test validation or research purposes. The computer took the engine speed signal from the dynamometer, the engine torque signal 
from the dynamometer's load cell, and the throttle position signal from the throttle positioning sensor; and it used them to continuously control the engine's throttle in order to achieve the FTP or SET set points. Some indirect engine control was also necessary to keep the coolant temperature, the engine oil temperature, the intake-air temperature, and the intake air-humidity levels compliant with the 40 CFR, Part 86, Subpart N. Figure 21 shows the $3 \mathrm{~B}$ modules that were used to convert analog sensor signals into the digital signals read by the test cell computer.

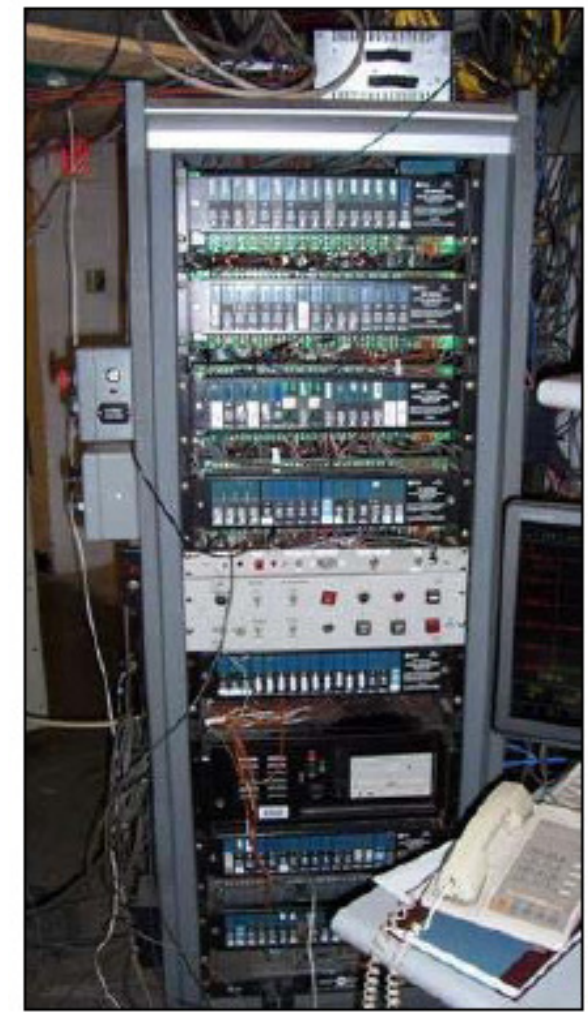

Figure 21 Test Cell DAQ

The 3B modules were also able to convert voltage signals into 5-20 mA currents that carried information such as engine speed and fuel flow rate to secondary data acquisitioning systems. 


\subsubsection{Dilution Tunnel}

A full-scale dilution tunnel was used, meaning that the entire flow of exhaust gas was continuously diluted. A full-scale dilution tunnel is valuable because it closely simulates ambient dilution conditions for the proper formation of DPM, it allows gaseous reactions to adequately complete, it provides sufficient mixing so that the diluted blend is uniform, and it eliminates the possibility of internal condensation (which can impair emissions instruments). Figure 22 shows where the conditioned air and raw exhaust meet at the mixing orifice and enter into the 18 inch dilution tunnel.

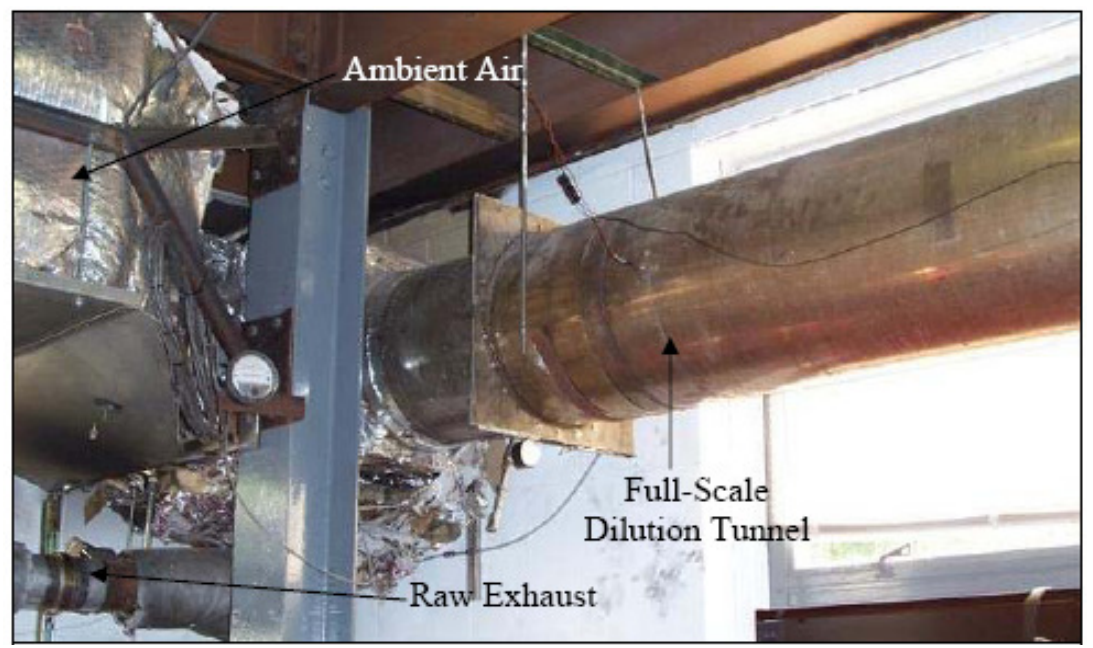

Figure 22 Dilution Location of Full-Scale Tunnel

Conditioned dilution air was fed to the dilution tunnel. Three critical flow venturis (CFV) (two $1000 \mathrm{scfm}$ and one $400 \mathrm{scfm}$ ) were placed just upstream of the blower to essentially create a constant volume sampler (CVS). The primary dilution ratio, given by equation 9 , changed according to the raw exhaust flow rate.

$$
R_{p}=\frac{Q_{\text {total }}}{Q_{\text {raw }}}
$$

Equation 9 
The raw exhaust flow rate, $Q_{\text {raw }}$, which typically ranged from about $120 \mathrm{scfm}$ to $830 \mathrm{scfm}$, dictated the primary dilution ratio because the total flow, $Q_{\text {total }}$, was held nearly constant by the CVS. The primary dilution ratio, $R_{p}$, was slightly affected by flow variance due to temperature effects on the CVS. The primary dilution ratio ranged anywhere from about 22 (at idle) to 2 (at full throttle). The standardized flow rate in the dilution tunnel was calculated using the following equation.

$$
Q_{\text {total }}=C_{\text {venturi }} *\left(\frac{p_{a b s}}{\sqrt{T_{a b s}}}\right) \quad \text { Equation } 10
$$

The absolute pressure before the venturis is given by $p_{a b s}$, the absolute temperature before the venturis is $T_{a b s}$, and $C_{\text {venturi }}$ is the calibration constant of the venturis. The flow rate was standardized at $29.92 \mathrm{in} \mathrm{Hg}$ and $68^{\circ} \mathrm{F}$.

\subsubsection{Fuel Measurement Systems}

To ensure data integrity, three different methods were used to determine fuel consumption. One method involved measuring the amount of carbon in the exhaust and using a fuel consumption equation that was based on fuel consumption/exhaust carbon proportionality. The fuel consumption equation method required the oxygen-to-carbon ratio of the fuel, the hydrogen-to-carbon ratio of the fuel, the specific gravity of the fuel, and the measured $\mathrm{HC}, \mathrm{CO}$, and $\mathrm{CO}_{2}$ masses in the exhaust.

A simple but accurate method to determine total fuel consumption over a test was to weigh the fuel before and after the test cycle. A fuel scale was used to determine the weight of the fuel and a 16 gallon stainless steel fuel tank at the start and end of each test. 
The fuel scale provided measurements accurate to $0.5 \%$ of the total fuel consumption over any test.

To measure continuous fuel flow, a Model 710 Max Machinery fuel conditioning system was used. The system held the fuel temperature at or below $109^{\circ} \mathrm{F}$ to meet 40 CFR, Part 86, Subpart N standards, and it consisted of fuel lines, a fuel meter, a fuel pump, and a heat exchanger. A digital signal of mass flow rate of fuel into the engine was sent from the fuel conditioning system to the DAQ. The specific gravity of each fuel was incorporated to report the measured mass.

\subsubsection{Gas Analyzers}

Exhaust gas analyzers are used at the CAFEE for engine emissions testing. The exhaust gas analyzers provided quality assurance in this experiment, because they gave indications of test repeatability. The exhaust gases measured during certification testing at the CAFEE are THC, $\mathrm{NO}_{x}, \mathrm{CO}$, and carbon dioxide $\left(\mathrm{CO}_{2}\right)$. A second analyzer was used to measure $\mathrm{NO}_{\mathrm{x}}$ at the outlet of the secondary dilution system. The purpose of having two $\mathrm{NO}_{\mathrm{x}}$ analyzers was to verify the secondary dilution ratio.

\subsubsection{Hydrocarbon Analyzer}

A Rosemount Analytical - Model 402 heated flame ionization detector (HFID) was used to measure THC emissions. A small flame within the analyzer is maintained by burning a $40 \%$ hydrogen/60 \% helium mixture. Hydrocarbons are ionized as they pass through the flame, and a set of polarized electrodes within the analyzer detect a resulting electrostatic field. An electric current is measured by the electrodes, and it is directly proportional to the concentration of THC in the exhaust sample. 


\subsubsection{Oxides of Nitrogen Analyzer}

A Rosemount Analytical - Model 955 chemiluminescence analyzer was used to measure $\mathrm{NO}_{\mathrm{x}}$ emissions. In a chemiluminescence analyzer, $\mathrm{NO}_{2}$ is first converted to $\mathrm{NO}$ and oxygen, so that all of the oxides of nitrogen are NO. Then, the NO is forced to react with ozone $\left(\mathrm{O}_{3}\right)$ to form $\mathrm{NO}_{2}$. About $10 \%$ of the total $\mathrm{NO}_{2}$ forms from $\mathrm{O}_{3}$; and $\mathrm{NO}$ is in the excited state, so it gives off energy in the form of light to reach its natural state once again. A photon detector within the analyzer continually senses the amount of light given off by chemiluminescence, which is proportional to the total amount of $\mathrm{NO}_{\mathrm{x}}$ in the sample.

\subsubsection{Carbon Monoxide Analyzer}

Two Horiba AIA-210 CO analyzers were used in order to measure a broad range of concentration. The Horiba AIA-210 is a non-dispersive infrared (NDIR) type analyzer that operates under the principle that the spectrum of infrared light absorbed by a sample is related to the sample's present gases. One $\mathrm{CO}$ analyzer was set to measure from 0 to $1000 \mathrm{ppm}$, and the other was set to measure 0 to $5000 \mathrm{ppm}$. Using two $\mathrm{CO}$ analyzers set to different ranges allowed for a more accurate interpretation of the data.

\subsubsection{Carbon Dioxide Analyzer}

A Horiba AIA-210 LE $\mathrm{CO}_{2}$ analyzer was used to measure $\mathrm{CO}_{2}$. The AIA-210 LE is a non-dispersive infrared (NDIR) analyzer, and it operates under the same basic principles as the AIA-210 CO analyzer.

\subsubsection{TEOM}

A Rupprecht and Patashnick series 1105 diesel particulate monitor TEOM sensor unit was used to continually measure PM mass rate in the dilution tunnel. The way in 
which the TEOM operates is explained in detail in the Literature Review section of this report. The TEOM was used in this study to relate the continuous PM mass flow through the tunnel to the approximate PM mass flow through the DMS. The TEOM can be seen in Figure 23.

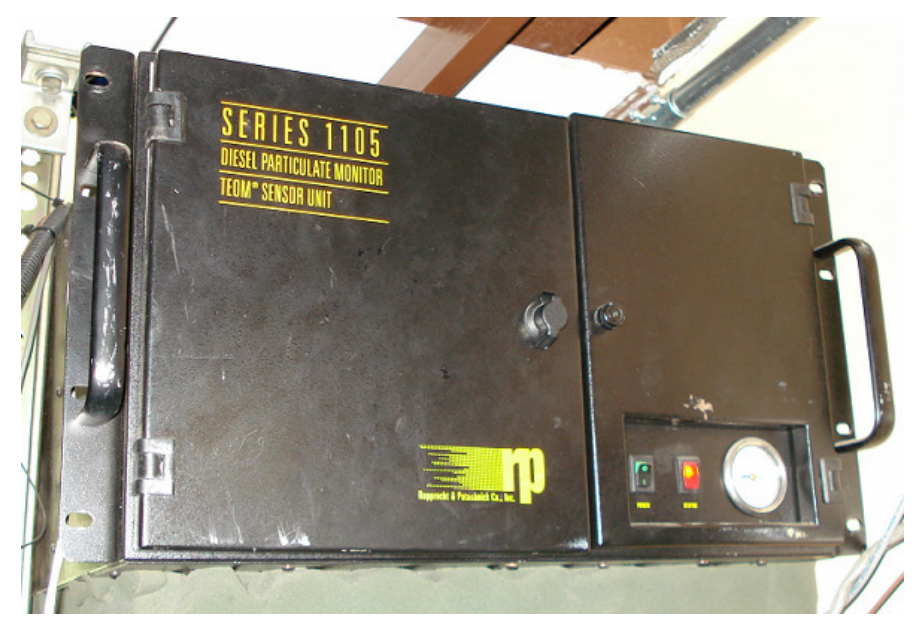

Figure 23 TEOM Sensor Unit

\subsubsection{Chiller}

Compressed air was used as the dilution air in the secondary dilution system. Since humidity of the diluted mixture affects the formation/destruction of DPM during secondary dilution, it was desired for the dilution air to be filtered and dehumidified. Air purity was achieved via a HEPA filter within the secondary dilution system. To dry the air, a Deltech HG25 Refrigerated Dryer was connected to the compressed air system. The chiller can be seen in Figure 24 on the following page. 


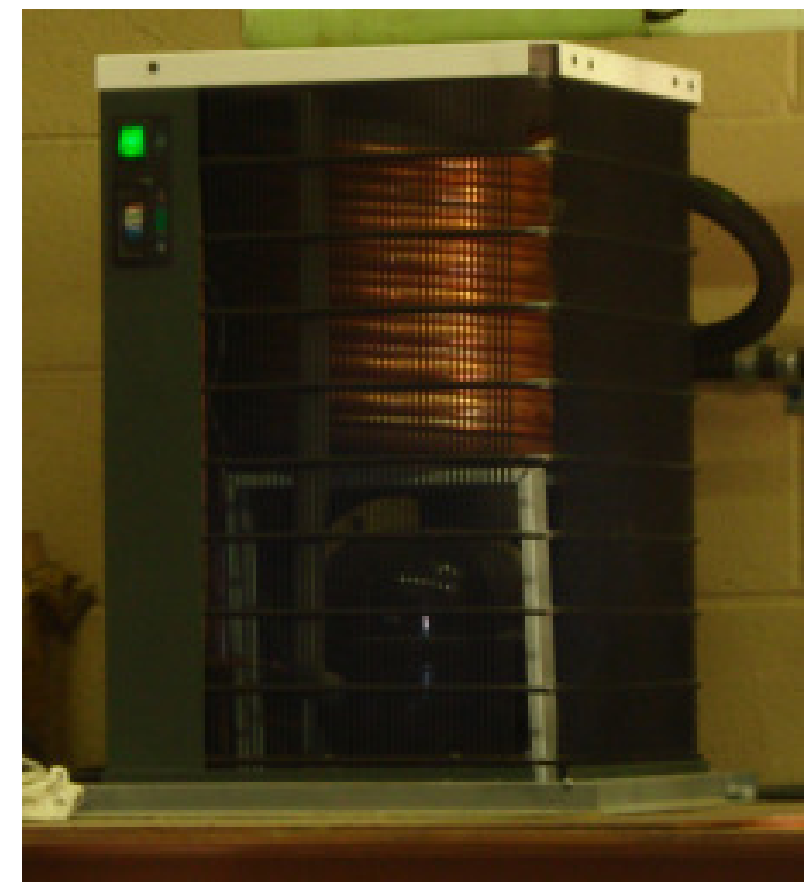

Figure 24 HG25 Refrigerated Dryer

\subsubsection{DMS-500}

A 2000 Cambustion DMS-500 was chosen for this study because of its ability to detect transient particle distributions. Basic operating principles of the DMS-500 are mentioned in the Literature Review section. An illustrated explanation of the key operating principle can be seen in Figure 25, below.

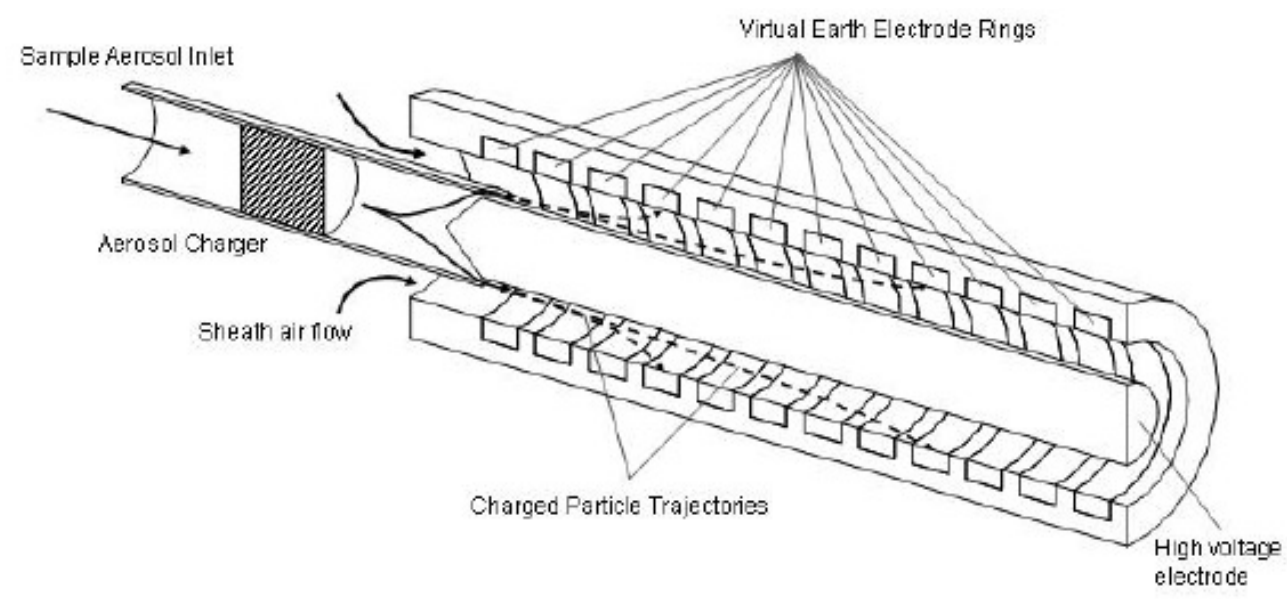

Figure 25 Differential Mobility Spectrometer [30] 
The diluted exhaust sample is drawn through the aerosol charger. Then the charged aerosol enters the classifier, which is surrounded by clean sheath flow. An electric field causes particles to drift toward the electrometers, or electrode rings. The charged particle trajectory and the ring on which a particle will land depend on drag and momentum (mass and charge). The denser the particle is, the farther it will travel within the DMS. The measurements of the DMS are consequently based on equivalent mobility diameter.

A remote impactor was attached to the sample line to remove excessively large particles. The DMS-500 required a vacuum pump, which was used to draw between 7.2 lpm and $8.8 \mathrm{lpm}$ of aerosol through the DMS and eventually expel sampled gases to the environment. A DAQ system was built into the DMS, so all that was required to log or review data was a PC. Figure 26 illustrates what a typical instantaneous aerosol measurement would look like.

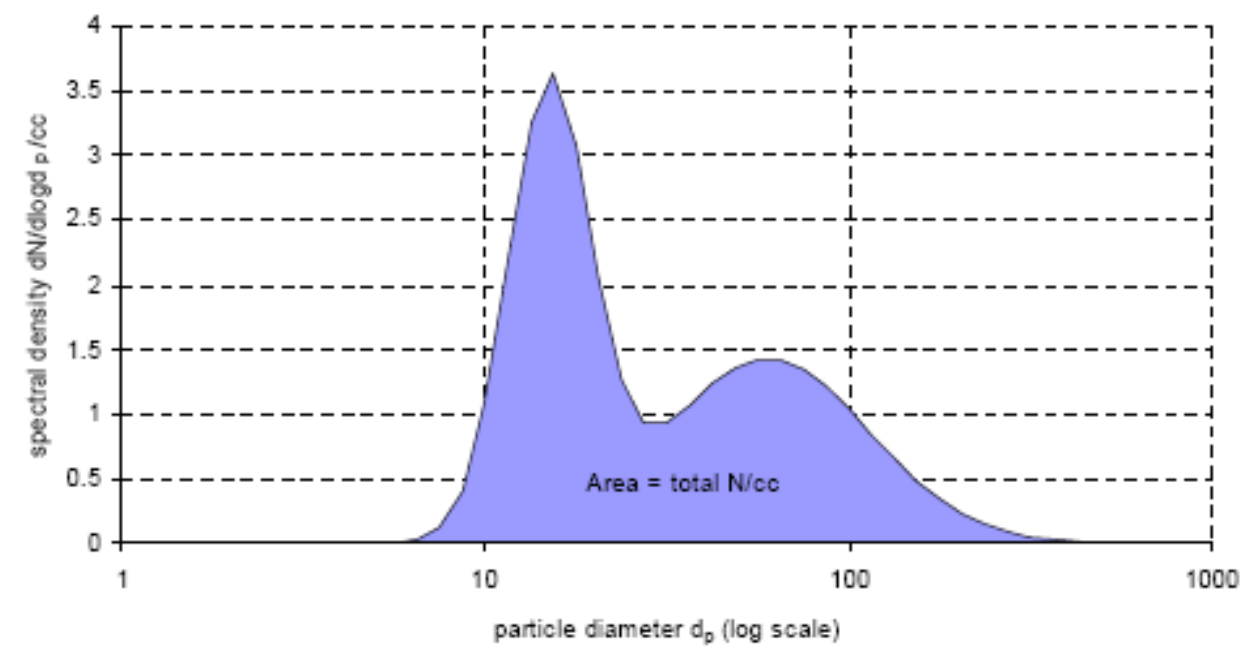

Figure 26 Particle Size Spectra [30]

Shown in Figure 27, the DMS-500 used in this study was one of the first beta models to be sold in the United States. Problems were often encountered with sample 
flow rate and signal noise due to soot build up within the instrument. After every FTP, it was necessary to restart the DMS and "zero" the electrodes' current signal using the PC, so that an accurate reading of zero could be the starting point for the next test. Cleaning the instrument, which consisted of removing the high voltage electrode and wiping off electrode rings, was necessary at least once daily (6-12 FTPs). The frequency of cleaning was inversely proportional to the overall dilution ratio.

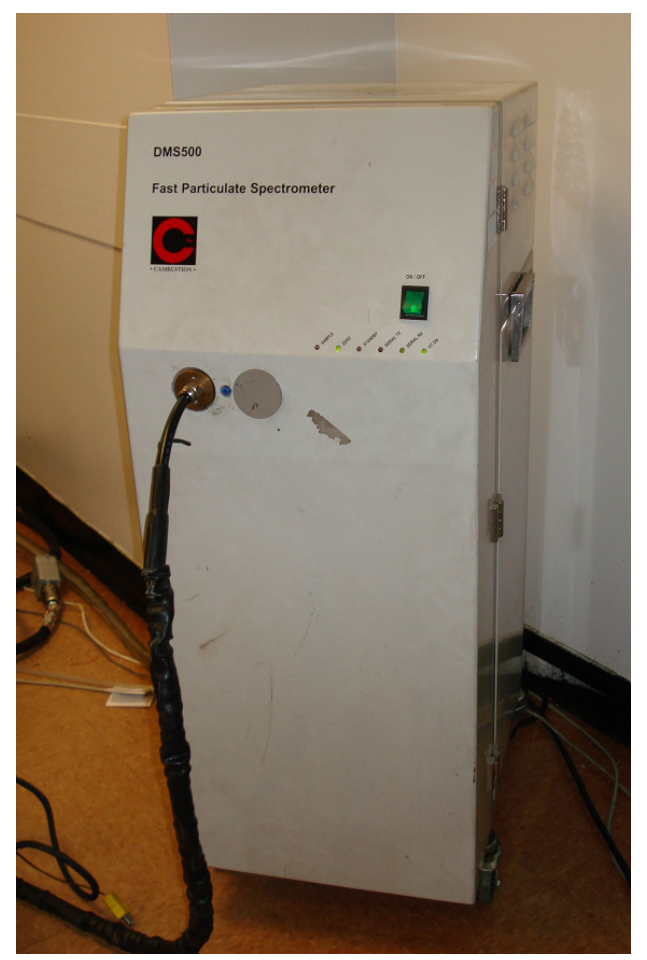

Figure 27 DMS-500

\subsubsection{Secondary Dilution System}

Initially, the aerosol sample was taken directly from the primary dilution tunnel. This caused the DMS to over range for several minutes during an FTP. Therefore, it was clear that the use of a secondary dilution system for the DMS-500 was essential. Several secondary dilution techniques were implemented in hopes that one would provide accurate and repeatable measurements. 
The first secondary dilution system was very simple and somewhat effective. It consisted of a rotometer that could limit the amount of compressed air being mixed in a stainless steel Tee with the cooled aerosol sample. The rotometer diluter made it nearly effortless to find a suitable dilution ratio for the tunnel/DMS combination. Once a dilution ratio was found that seemed to provide the DMS with a measurable range of concentrations, data was taken from several FTPs. The repeat tests with the rotometer were poorly comparable, because the dilution ratio was not sufficiently constant. Although the volumetric flow rate of compressed air through the rotometer remained approximately the same, fluxuations in ambient temperature and air pressure caused the mass flow rate of air into the Tee to vary significantly. From the rotometer experiments, an optimal dilution ratio was discovered, and it became apparent that the use of a heated and pressure regulated dilution system was necessary.

The next step was to try a heated dilution box, shown in Figure 28, that used a mass flow meter and a mass flow controller to continually adjust the mass flow rate of dilution air in order to provide a constant dilution ratio.

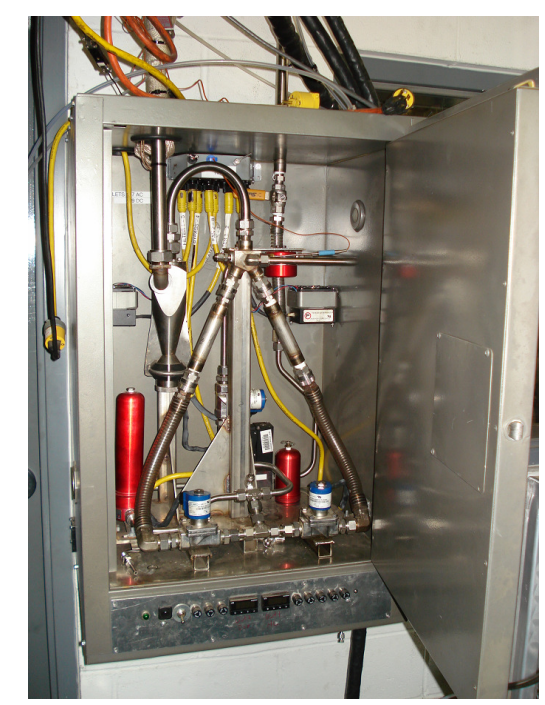

Figure 28 Mass-Flow-Controller Dilution System 
The mass flow controller box was conventionally used for the secondary dilution in gravimetric PM filter applications, so the average dilution ratio of each test was within a few percent of the user-specified dilution ratio. A major problem however, was the fact that the system was ineffective in maintaining a constant dilution ratio throughout an FTP. The mass flow controller was unable to respond quickly and accurately enough to follow the transitions in the FTP, and the resulting DMS data was not generally repeatable.

Finally, a heated ejector diluter was custom built to provide the DMS-500 with an aerosol sample from the main dilution tunnel at a constant secondary dilution ratio. Figure 29 is a schematic of the heated ejector diluter.

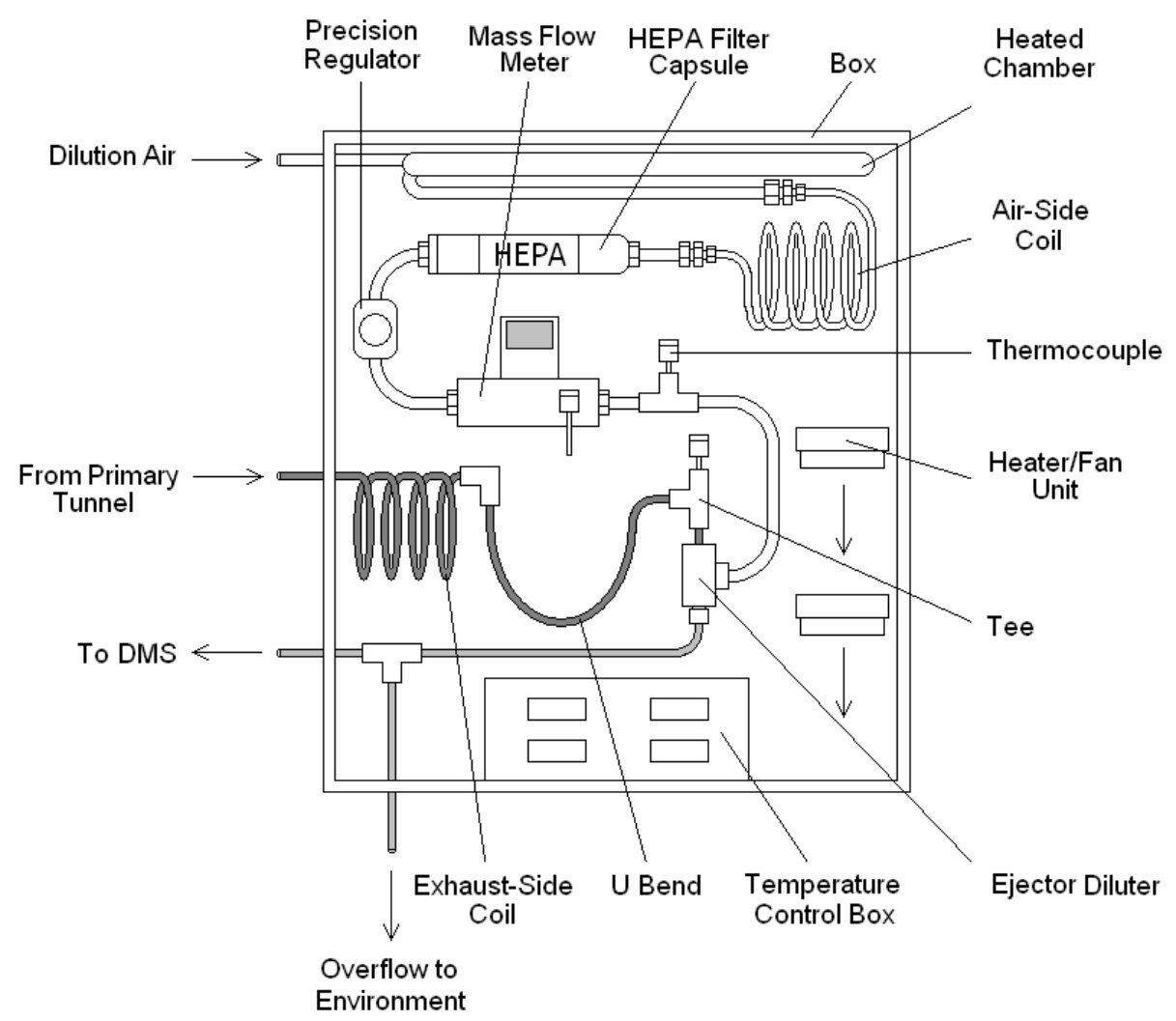

Figure 29 Secondary Dilution System Design 
The ejector dilution system was designed so that a user can take out and replace any single part without having to remove any other components. With the heater/fan units show above, the interior of the dilution box is maintained at $47+-1.0^{\circ} \mathrm{C}$ (the same temperature that is used for the 2007 gravimetric filter systems specified in 40CFR86 Subpart N). The heated chamber, as labeled above, heats the compressed air to the same temperature, $47+-1.0^{\circ} \mathrm{C}$. A heated sample line, not shown in the figure above, stretches from the main dilution tunnel to the dilution box and maintains the sample temperature at $47+-1.0{ }^{\circ} \mathrm{C}$. The heated sample line and the heated chamber are not precise enough to keep the sample and air temperatures exactly the same, so the air side and exhaust side coils are there to preserve equilibrium between the two temperatures and the box's interior temperature. The temperature control box, along with three thermocouples (one on the air side of the ejector diluter, one on the sample side of the ejector diluter, and one on in the center of the box), controls the heaters for the heated chamber, heated sample line, and heater/fan units. A HEPA capsule filter prevents particles of the compressed air supply from entering the system. The precision regulator uses bleed off valves to keep the pressure from the compressed air supply to the ejector diluter constant. The ejector diluter uses the pressure and flow of the compressed air to create a vacuum that draws in the aerosol sample. Once the aerosol and compressed air mix in the ejector diluter, some of the diluted sample is sent to the DMS while the rest is evacuated to the ambient. The ejector diluter consists of two parts; an air operated vacuum pump and a critical orifice. The air supply pressure is directly proportional to the resulting vacuum pressure. As long as the vacuum pressure is greater than 16 in $\mathrm{Hg}$, the critical orifice ensures a constant flow rate of sample through the diluter. The dilution ratio can be adjusted, to a certain 
extent, by varying the air supply pressure (which changes the flow rate of dilution air) at the precision regulator. For greater adjustment of the dilution ratio, the critical orifice can be substituted with another critical orifice of greater or lesser flow. With adjustment to the regulator and changing of the critical orifice (orifices ranging from $2 \mathrm{lpm}$ to 15 $\mathrm{lpm}$ ), a constant dilution ratio can theoretically be set at anywhere from 7.80 to 67.5 . The mass flow meter continually monitors air flow and pressure into the ejector diluter for quality assurance and data acquisition purposes only. The $U$ bend, on the sample side of the ejector diluter, was designed so that the sample line can easily be disconnected from the ejector diluter for changing of the critical orifice. The box itself is large enough to accommodate future accessories, and it has a removable mounting panel so that all parts can be easily installed or removed.

Properly maintaining the temperatures of the aerosol sample and dilution air is crucial. An increase or decrease of $3{ }^{\circ} \mathrm{C}$ on either side of the ejector diluter can cause a change in dilution ratio of about $2 \%$. The ejector dilution system's ability to maintain a constant dilution ratio depends mostly on: the heaters' ability to maintain constant temperature, the regulator's ability to maintain constant pressure, the orifice's ability to maintain constant sample flow, and the vacuum pump's ability to maintain sufficient vacuum pressure and constant air flow. As discussed in the Secondary Dilution Ratio Validation section of this thesis, the secondary dilution ratio was calculated using two $\mathrm{NO}_{\mathrm{x}}$ analyzers during several SETs. The combined results yield a secondary dilution ratio with an average value of 24.05, a maximum value of 25.32 (5.28 \% higher), and a minimum value of 23.06 (4.12\% lower). The ability of the secondary dilution system to maintain a constant dilution ratio may actually be better than indicated, because variation 
in $\mathrm{NO}_{\mathrm{x}}$ measurements is included in the calculation of the maximum and minimum values. Detailed calculations involving the dilution system's overall precision can be found in the Secondary Dilution Ratio Validation section on page 169. The final form of the ejector dilution system is presented below in Figure 30.

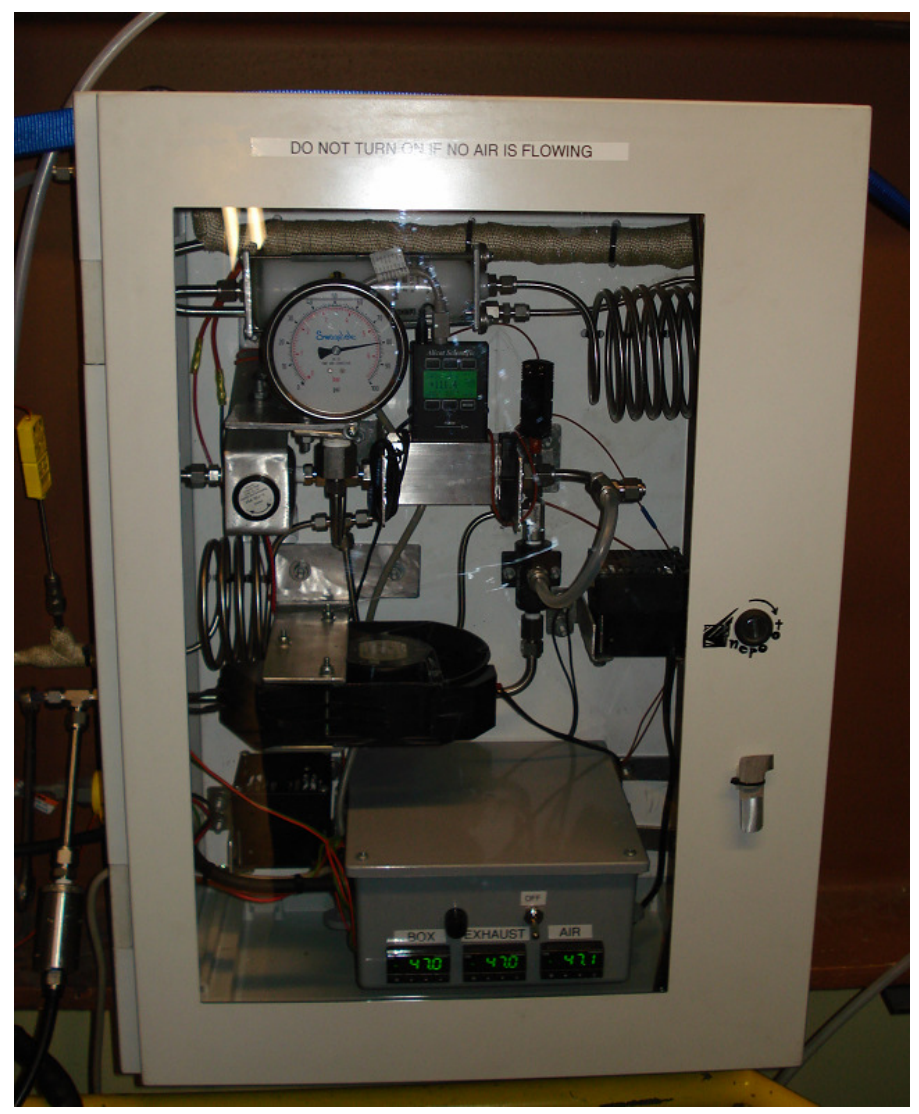

Figure 30 Heated Ejector Diluter

The Alikat Scientific M Series mass flow meter within the dilution system was calibrated using the Heise PTE-1 pressure sensor and the Marium Instruments laminar flow element (LFE) shown in Figure 31. The Heise pressure sensor was also used to determine the barometric pressure during each engine test cycle. 


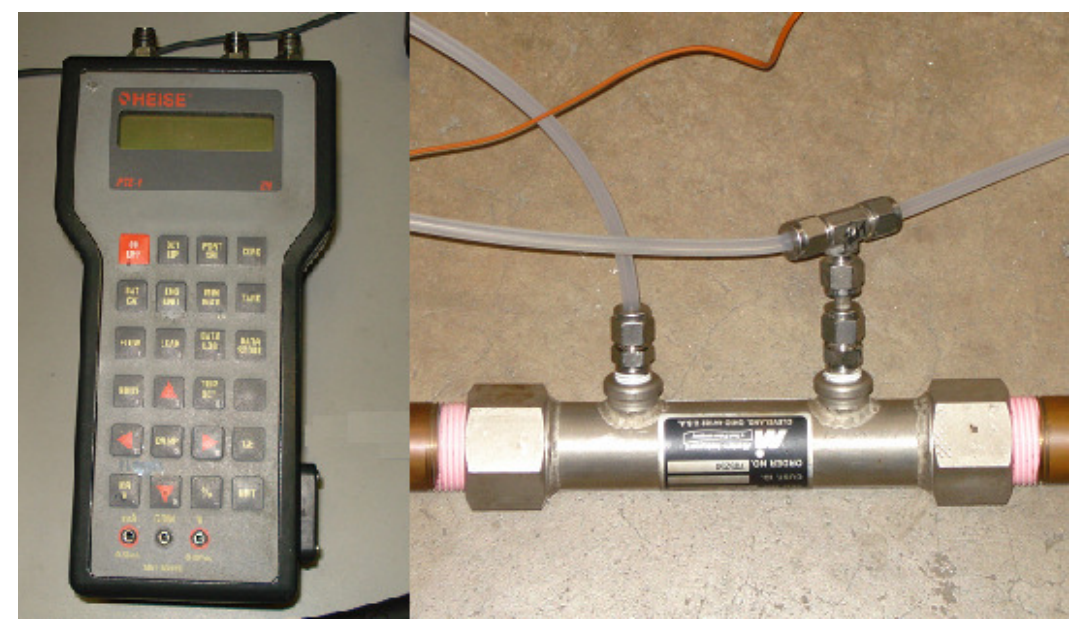

Figure 31 Pressure Sensor and LFE

The results of the mass-flow meter calibration are shown below in Table 6 .

Table 6 Mass-Flow Meter Calibration Results

\begin{tabular}{|c|c|c|}
\hline $\begin{array}{c}\text { Laminar Flow } \\
\text { Element (slpm) }\end{array}$ & $\begin{array}{c}\text { Flow Meter } \\
(\text { slpm) }\end{array}$ & $\begin{array}{c}\text { Difference } \\
(\%)\end{array}$ \\
\hline 28.49 & 28.10 & 1.36 \\
\hline 40.58 & 40.10 & 1.18 \\
\hline 53.09 & 52.40 & 1.31 \\
\hline 65.44 & 64.70 & 1.13 \\
\hline 77.99 & 77.10 & 1.14 \\
\hline 90.73 & 89.50 & 1.35 \\
\hline 103.29 & 101.90 & 1.34 \\
\hline 116.50 & 114.50 & 1.72 \\
\hline 128.67 & 127.10 & 1.22 \\
\hline
\end{tabular}

For the range of mass-flow rates measured, the maximum difference between the Alikat mass-flow meter and the Marium Instruments laminar flow element was $1.72 \%$ at 116.5 slpm. The minimum difference was $1.13 \%$ at 65.44 slpm. None of the calibration points were off by more than $2 \%$, so adjustment to the mass-flow meter was not necessary. 


\subsection{SAMPLING PROCEDURE}

\subsubsection{Procedure for FTP}

The procedure in which data was collected was consistent from test to test. Before any test, the heated ejector diluter was given at least one hour to stabilize its internal temperature. The precision regulator pressure was always set to $80 \mathrm{psi}$, which resulted in a constant secondary dilution ratio of $\sim 24$. The DMS also required time to warm up, and it was given at least forty five minutes before each test session. The electrometer rings within the DMS were "zeroed" about five minutes before the start of each FTP so that there would be as little time as possible for drift to occur. The DMS drew its sample via an external vacuum pump, which was turned on about a minute after the DMS was powered up. When necessary, orifice plates within the remote impactor on the DMS sampling line were changed in order to maintain a sample flow of $8 \mathrm{lpm}+-10$ \%. In the time between FTPs, the DMS was reset and the rings were rezeroed. Data logging on the DMS's data acquisition system began about one minute before the start of each FTP and was over about one minute after the end. At the end of each day, the electrometer rings were cleaned with isopropyl alcohol and "chem wipes." The rest of the laboratory, which includes the exhaust gas analyzers, the $70 \mathrm{~mm}$ PM filters, and the TEOM, was run by a test-cell engineer. The full-scale dilution tunnel maintained an average flow of approximately $2400 \mathrm{scfm}$, which resulted in main-tunnel dilution ratios of about 22 at idle and 2 at full throttle. Therefore, the overall dilution ratio at the intake of the DMS ranged from about 480 to 48 during each FTP. Data was collected from FTPs with the DMS in order to study the detectable changes in PM formation due to: fuel additives, day-to-day variation, and temperature of the heated sample line. FTP data 
was taken with the TEOM in an attempt to relate continuous PM mass data to the continuous PM concentration data from the DMS.

\subsubsection{Day-to-Day Variation Testing}

To help determine the typical day-to-day variation in PM formation, five nominal fuels were tested on multiple days. The first fuel tested for day-to-day variation was a biodiesel fuel named NBB Ref., and it was run over two hot-start FTPs through the 2004 Cummins ISM 370 engine on two consecutive days. The second fuel was tested in the same engine over the same two days. It was NBB-B05, and it was used during two hotstart FTPs on each day. The third fuel tested on two consecutive days was RF-71, and it was run for two hot-start FTPs on the non-rebuilt 1992 Detroit Diesel DDC S60 engine. The fourth fuel was TCEQ Tex LED Ref., and it was tested during three hot-start FTPs on each day for three non-consecutive days on the 1992 Detroit Diesel DDC S60 engine. The fifth fuel tested for day-to-day variation was also run through the 1992 Detroit Diesel DDC S60 engine. It was tested for two consecutive days, over four hot-start FTPs on each day. The fuels and engines tested for day-to-day variation were arbitrarily chosen so that a broad range of results could be examined.

\subsubsection{Fuel Additives Testing}

Reference fuels were evaluated using FTPs, then one to nine fuel additives were used in attempts to find emissions advantages. To study how fuel additives effect continuous PM concentration, six different sets of reference/additive fuel combinations were tested and analyzed using the DMS. The first set of reference/additive tests was run on the 2004 Cummins ISM 370 engine, and it consisted of one biodiesel reference fuel (NBB Cand.) and two biodiesel additive fuels (NBB Ref. and NBB-B05), where three 
hot-start FTPs were run on each fuel. The second set consisted of 30 FTPs, and it was run on the rebuilt 1992 Detroit Diesel DDC S60 engine. It included one low-cetane reference fuel (LC) and nine low-cetane additive fuels (LC trt. 2-10), where three hotstart FTPs were run for each fuel. The third set of reference/additive tests was run on the rebuilt 1992 Detroit Diesel DDC S60 engine, and it was made up of one reference fuel (PONCA) and one additive fuel (PONCA trt. 1), where only two hot-start FTPs were run on each fuel. The fourth set was run on the rebuilt 1992 Detroit Diesel DDC S60 engine, and it included one reference fuel (Trainer) and two additive fuels (50/50 Trainer Cand. and 50/50 Trainer Cand. trt. 1), where two hot-start FTPs were run off of each fuel. The fifth set of tests was run on the rebuilt 1992 Detroit Diesel DDC S60 engine. It was made up of one reference fuel (LC) and two additive/biodiesel blend fuels (LC-B10A and LCB20A), and three hot-start FTPs were run on each fuel. The last set of reference/additive tests was also run on the rebuilt 1992 Detroit Diesel DDC S60 engine, and it consisted of one reference fuel (FEM) and two additive fuels (FEM trt. 6 and FEM trt. 7), where three hot-start FTPs were run with each fuel. The reference/additive fuel research was done during several different predetermined test programs, so the resulting PM data that was collected was made up of random sets of fuel/additive combinations.

\subsubsection{Unheated Sample Line Testing}

To see what effects on PM formation there might be, the temperature of the heated sample line for the secondary ejector dilution system was varied. During two identical hot-start FTPs, the heated sample line was turned off. During the next three hotstart FTPs of the same fuel (2007 CP Cert.), the heated sample line was set at $47{ }^{\circ} \mathrm{C}$. All five tests were completed on the same day with the 1992 Detroit Diesel DDC S60 engine. 


\subsubsection{TEOM and Gravimetric Filters}

During most of the FTPs that were assessed with the DMS-500, PM mass data was being collected with $70 \mathrm{~mm}$ PM filters and the TEOM. The TEOM was located just outside of the main dilution tunnel near the gaseous sampling plain, and its oscillating filter was changed after each set of tests. Data from the TEOM was collected using the laboratory's central DAQ. The sampling technique for the $70 \mathrm{~mm}$ PM filters is described in depth in the Literature Review of this thesis in the EPA Standards and Regulation section. TEOM, DMS, and filter data was taken simultaneously so that a correlation in PM mass between the three instruments could be observed.

Continuous PM data from over 120 FTPs was collected using the DMS-500. Table 7 displays the array of engines and fuels that were tested over numerous hot-start FTPs. 
Table 7 Hot-Start FTPs - Examined Fuels for Each Test Engine

\begin{tabular}{|c|c|c|c|c|c|c|c|}
\hline & Engine & \multicolumn{2}{|l|}{ DDC Series 60} & \multicolumn{2}{|c|}{ Rebuilt DDC Series 60} & \multicolumn{2}{|c|}{ Cummins ISM 370} \\
\hline \multirow{21}{*}{ Fuel } & \multirow{21}{*}{ [Number of Runs] } & Grad. Ref. & [3] & 2007 CP Cert. & [5] & NBB Cand. & [3] \\
\hline & & LC & {$[6]^{*}$} & RF 71 & {$[6]^{*}$} & B10 & [2] \\
\hline & & LC trt. 2 & [3] & CF 16 trt. 1 & [3] & NBB Ref. & {$[5]$ * } \\
\hline & & LC trt. 3 & [3] & CF 16 trt. 4 & [3] & NBB-B05 & {$[6]^{*}$} \\
\hline & & LC trt. 4 & [3] & TCEQ Tex LED Ref. & {$[9]^{*}$} & & \\
\hline & & LC trt. 5 & [3] & Candidate & {$[8]^{*}$} & & \\
\hline & & LC trt. 6 & [3] & & & & \\
\hline & & LC trt. 7 & [3] & & & & \\
\hline & & LC trt. 8 & [3] & & & & \\
\hline & & LC trt. 9 & [3] & & & & \\
\hline & & LC trt. 10 & [3] & & & & \\
\hline & & PONCA & [2] & & & & \\
\hline & & PONCA trt. 1 & [2] & & & & \\
\hline & & Trainer & {$[2]$} & & & & \\
\hline & & 50/50 Trainer/Cand. & [2] & & & & \\
\hline & & 50/50 Trainer/Cand. trt. 1 & [2] & & & & \\
\hline & & LC-B10A & [3] & & & & \\
\hline & & LC-B20A & [3] & & & & \\
\hline & & FEM trt. 3 & [3] & & & & \\
\hline & & FEM trt. 6 & [3] & & & & \\
\hline & & FEM trt. 7 & [3] & & & & \\
\hline
\end{tabular}

\subsubsection{Procedure for SET}

The only difference in DMS operation requirements for the SETs, as opposed to for the FTPs, was the need to clean the DMS more frequently. Instead of the electrometer rings being cleaned once a day, they were cleaned once they accumulated too much soot to be properly zeroed. Continuous PM data was taken from SETs with the DMS with hopes to observe the detectable differences in PM formation due to changes in relative humidity within the main dilution tunnel, fuel type, and overall dilution ratio. 


\subsubsection{Humidity Testing}

It was suspected that slight changes of the relative humidity within the dilution tunnel were having an effect on the PM measurements taken by the $70 \mathrm{~mm}$ gravimetric filters. To develop supporting evidence, the humidity level within the main dilution tunnel was varied for a few sets of tests, and continuous PM distribution measurements were taken with the DMS. Two gate valves were connected to a steam line, and it was routed directly to the ambient air intake of the full-scale dilution tunnel. The use of two gate valves ( 2 in. and 0.5 in.) on the steam line allowed for fine tuning of the relative humidity within the dilution tunnel. For this particular experiment, nine SETs were performed at three different humidity levels. The first three SETs were run at the lowest relative humidity level, $37 \%$. The second set of three SETs was performed at a medium humidity level of $58 \%$. The third group of three SETs was run with a humidity level of $88 \%$. On the specific day that the humidity testing was done, $37 \%$ was the lowest possible relative humidity value that could be achieved with the available resources. All nine of the humidity specific SETs were run using the Guttman fuel and the rebuilt 1992 Detroit Diesel DDC S60 engine.

\subsubsection{Fuel Type Testing}

For the sake of determining exactly how distinguishable the differences are in PM formation due to fuel type, PM data was taken with the DMS from thirteen SETs of twelve different fuels. Some of the tests were performed on the same day, and others weren't. All of the twelve fuels used for this fuel-type related evaluation were run on the rebuilt 1992 Detroit Diesel DDC S60 engine, and they were: LC, LC trt.2-10, LC-B10A, 
and LC-B20A. The fuels tested for this were all low cetane fuels, and they were part of separate test programs.

\subsubsection{Dilution Ratio Testing}

To aid the understanding of the effects that overall dilution ratio has on PM formation, measurements were taken with the DMS from various SETs of different dilution circumstances. Orifices within the CVS were strategically changed so that the flow rate of the main dilution tunnel would be different for each of three SETs. There was one SET with an average main-tunnel flow of $\sim 1400 \mathrm{scfm}$, one SET with an average flow of $\sim 2400 \mathrm{scfm}$, and one SET with an average flow of $\sim 3400 \mathrm{scfm}$. Since the flow of the main dilution tunnel was different for each test, and the engine's continuous exhaust flow was approximately the same for each test; the primary dilution ratio was at three significantly different settings. During the SET that was run at $1400 \mathrm{scfm}$, the main dilution ratio ranged from about 13 at full throttle to about 1.2 at idle. For the SET that was performed with a 2400 scfm tunnel, the main dilution ratio ranged from about 20 at idle to about 2 at full throttle. The SET that incorporated a tunnel flow of $3400 \mathrm{scfm}$ had a primary dilution ratio of about 31 at idle and about 2.8 at full throttle. All three of these SETs were completed with the Guttman fuel and the rebuilt 1992 Detroit Diesel DDC S60 engine. The secondary dilution ratio of about 24 was verified with the use of two $\mathrm{NO}_{\mathrm{x}}$ analyzers; one before and one after the secondary dilution system. 
Data was collected with the DMS from 25 SETs. Table 8, below, shows the engines and fuels that were tested over various SETs.

Table 8 SET: Examined Fuels

\begin{tabular}{|c|c|c|c|c|}
\hline Engine & Fuel & Number of Runs & $\begin{array}{l}\text { Approx. Tunnel } \\
\text { Flow (scfm) }\end{array}$ & $\begin{array}{l}\text { Approx. Relative } \\
\text { Humidity }(\%)\end{array}$ \\
\hline \multirow[t]{18}{*}{ DDC Series 60} & LC & 1 & 2400 & 50 \\
\hline & LC trt. 1 & 1 & 2400 & 50 \\
\hline & LC trt. 2 & 1 & 2400 & 50 \\
\hline & LC trt. 3 & 1 & 2400 & 50 \\
\hline & LC trt. 4 & 1 & 2400 & 50 \\
\hline & LC trt. 5 & 1 & 2400 & 50 \\
\hline & LC trt. 6 & 1 & 2400 & 50 \\
\hline & LC trt. 7 & 1 & 2400 & 50 \\
\hline & LC trt. 8 & 1 & 2400 & 50 \\
\hline & LC trt. 9 & 1 & 2400 & 50 \\
\hline & LC trt. 10 & 1 & 2400 & 50 \\
\hline & LC-B10A & 1 & 2400 & 50 \\
\hline & LC-B20A & 1 & 2400 & 50 \\
\hline & Guttman & 3 & 2400 & 37 \\
\hline & Guttman & 4 & 2400 & 88 \\
\hline & Guttman & 3 & 2400 & 60 \\
\hline & Guttman & 1 & 3400 & 50 \\
\hline & Guttman & 1 & 1400 & 50 \\
\hline
\end{tabular}




\section{RESULTS AND DISCUSSION}

\subsection{INTRODUCTION}

The core objective of this research was to determine the minimum detectable differences in particle concentrations that were significant between engine operating conditions. Among several other objectives was to study some of the reasons for these differences. The subsequent results are discussed and presented in a manner that enables the objectives of this research to be achieved.

\subsection{METHODOLOGY}

The effective density and water adsorption equations used for PM mass comparison between the TEOM and the DMS-500 were found through research, and they can be reviewed in the Literature Review section of this thesis. The equations used for the rest of the calculations are explained throughout the Results and Discussion section. Most of the PM data has been converted to brake-specific information, so that the comparisons of operating conditions would be standardized. Continuous particle concentration plots (contour plots) generated by the original DMS software were not alterable, so they were provided on a spectral density basis ((dN/dlogdp)/cc). The concentrations given by the contour plots are for the twice-diluted sample. All brakespecific particle concentrations presented in the results are lower than their actual values by a factor of 1.5 (the secondary dilution ratio of 24 and the factor of $1 / 16$ for the conversion of spectral density to particles per cubic centimeter yield 24/16, or 1.5) [30]. Correction for these values was unnecessary, because they were compared on a percentage basis. Important test-cell data, such as: fuel consumption, exhaust gas concentrations, primary tunnel flow, PM mass rate, primary dilution air humidity, engine 
speed, and brake power were combined in a single excel file with the continuous PM size and concentration data from the DMS. Two methods of time alignment were used for the analysis of the data. To align the test-cell data with the DMS data, engine speed was plotted against the continuous concentration of $100 \mathrm{~nm}$ particles and the peaks were matched. Engine speed was also used to align the combined test-cell/DMS data from separate runs. With use of the DMS sampling software, a 5 point (1 second) moving average was incorporated into the data acquisition process to smooth the $5 \mathrm{~Hz}$ DMS data. Excluding some of the humidity results, Microsoft Excel was used for the analysis of all of the following data. MATLAB ANOVA was used in some of the humidity results to contrast multiple 13-mode SETs.

In all of the following plots and charts where particle size, or particle diameter, is considered, the spectrum is not simply binned into size classes. The concentrations for each size were approximated using the trapezium rule (see DMS-500 manual) [30]. The line charts below are based on an average concentration spectrum over a specified period of time. The average-based plots in the FTP Results section were created using the average number of particles of each size over the entire test. The average-based plots in the SET Results section were produced from the average number of particles of each size during the last 10 seconds of each mode.

\subsection{FTP RESULTS}

\subsubsection{Day-to-Day Variation}

The coefficient of variation (COV) line in Figure 32 represents the run-to-run variation of three repeat hot-start FTPs, and it is a percentage of the total particle concentration of each diameter. The COV of each particle size was calculated by 
dividing the standard deviation by the average concentration and multiplying by one hundred. The three repeat tests of Figure 32 are represented by test numbers E01927_02, E01927_03, and E01927_04. The E01927 test sequence was performed using the 2004 Cummins ISM 370 with the NBB-B05 biodiesel blend fuel. The highest COV in Figure 32 was about $30 \%$, and it occurred in the $9 \mathrm{~nm}$ diameter particles. Particles within the range of $25 \mathrm{~nm}$ to $520 \mathrm{~nm}$ displayed COVs below $5 \%$.

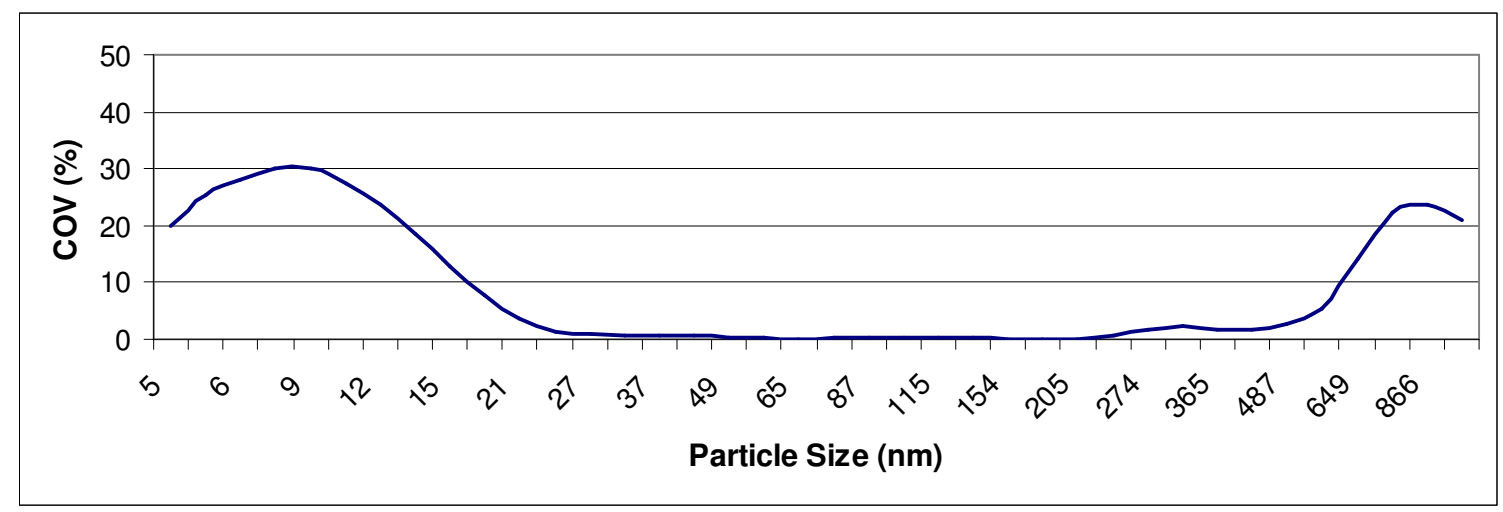

Figure 32 Run-to-Run Variation of the E01927 Test Sequence

Figure 33 is a similar COV plot for three repeat tests on the same engine and the same fuel on the following day. The results are quite different in that the maximum COV value occurs at $1000 \mathrm{~nm}$ and is about $50 \%$, the range of particles with a COV below $5 \%$ is between $18 \mathrm{~nm}$ and $205 \mathrm{~nm}$, and the COV for particles smaller than $18 \mathrm{~nm}$ is mostly below $10 \%$. As quantitatively discussed in the results of the Extremes of Run-to-Run Variation section, the ISM 370 typically provides poor test repeatability. The differences in run-to-run variation from day 1 to day 2 might have been due to the ECU's inability to provide consistent engine operation. 


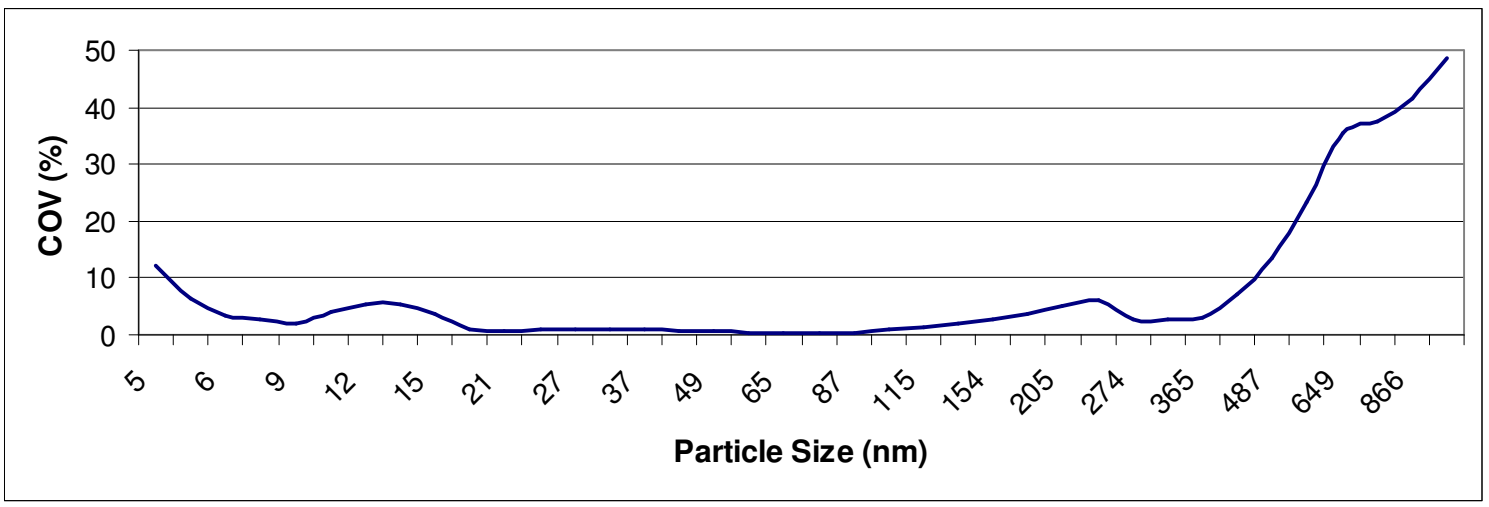

Figure 33 Run-to-Run Variation of the E01928 Test Sequence

The overall variation for the six tests from the E01927 test sequence and the E01928 test sequence is illustrated below in Figure 34. Since three hot-start FTPs were performed on each day, it is evident, but not conclusive, that a similar trend of variation would occur if more tests were administered on each day for more days.

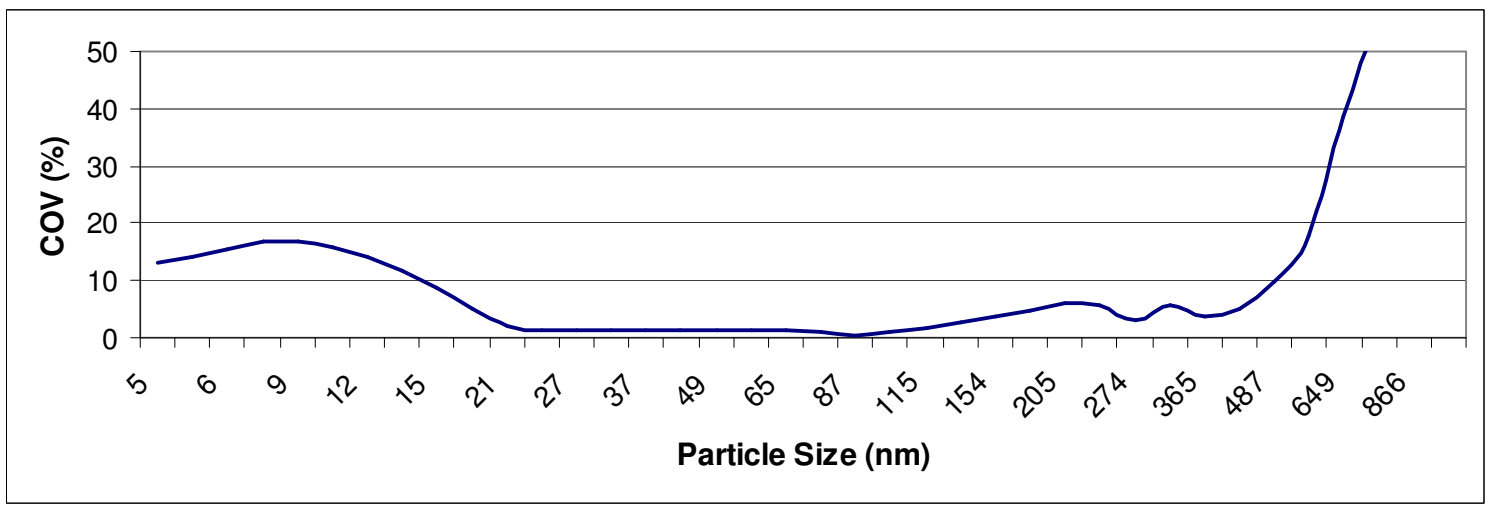

Figure 34 Day-to-Day Variation of the 2004 Cummins ISM 370

The average concentrations of each particle size are displayed for day 1 and day 2 in Figure 35. The dashed blue line represents the average number of particles per unit work for the E01927 test sequence, and the dashed green line shows the same for the E01928 sequence. The solid blue and green lines illustrate standard deviations for each set of tests. Anywhere the standard deviation lines from the two sequences do not overlap, there is considered to be a significant difference from one day to the next. This 
basic form of statistical analysis was chosen to allow comparable results between statistically small sets of data. From inspection of Figure 35, it can be seen that the concentration spectrums are similar, and it is difficult to observe any significant differences between the results of day 1 and day 2. The 2004 ISM 370 is the only of the three engines that consistently produced a flat line between the average number of particles for the nuclei mode and the accumulation mode. Its unique trend line could possibly be attributed to the EGR system's impact on the formation of particles.

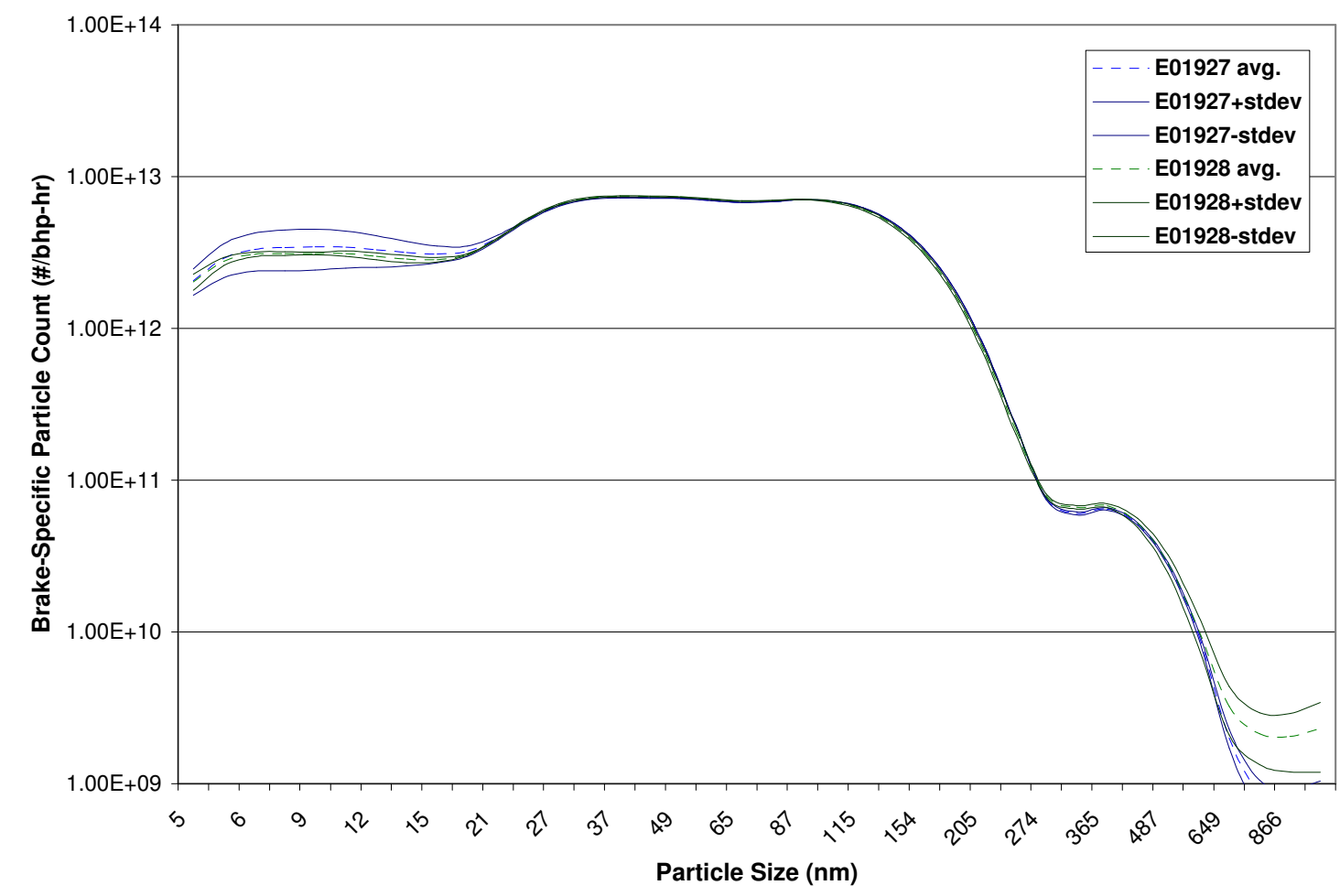

Figure 35 Particle Concentration Comparison of E01927 and E01928 Test Sequences

Figure 36 displays the differences between day 1 and day 2 on a percent basis. Due to rounding, the number 6 appears twice as a particle size. Dark blue columns represent differences that are significant, while light blue columns represent differences that are not. The columns in which the error bars do not cross the $\mathrm{x}$-axis are considered 
significant. Differences were calculated using averages from day 1 and day 2 , and error bars were calculated by adding the COVs from day 1 to the COVs from day 2. Particle sizes that do not have overlapping standard deviations in Figure 35 are the same sizes that have dark blue columns in Figure 36. The differences from $750 \mathrm{~nm}$ to $1000 \mathrm{~nm}$ are not considered significant, because the error bars are greater than $50 \%$. The differences from day 1 to day 2 for this fuel and engine are significant at around $4 \%$ in the $32 \mathrm{~nm}$ to $75 \mathrm{~nm}$ range and $3 \%$ to $10 \%$ in the $115 \mathrm{~nm}$ to $316 \mathrm{~nm}$ range. The significant day-to-day differences in this case were likely caused by inconsistent control from the ECU and particle formation issues with EGR.

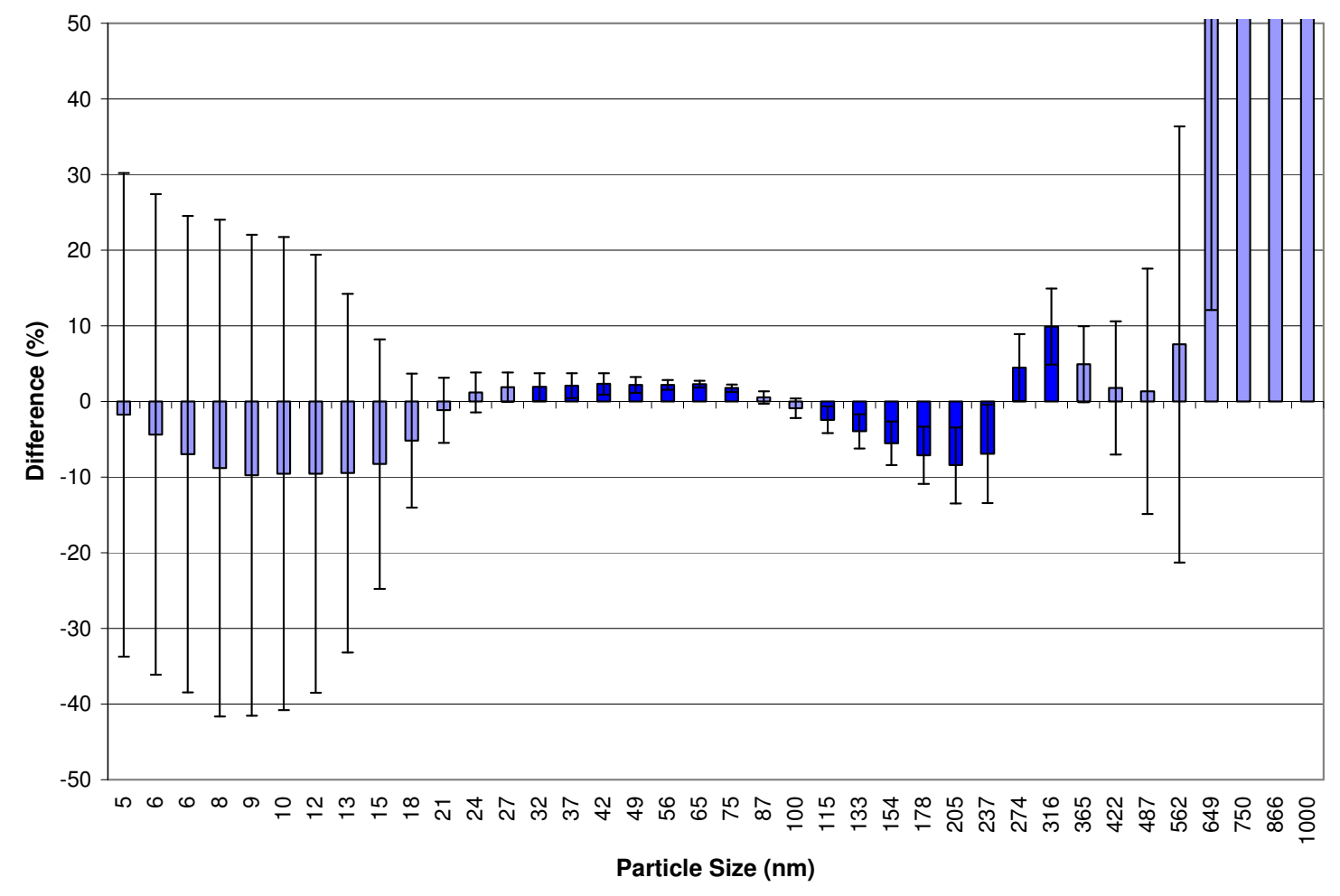

Figure 36 Particle Concentration Differences between E01927 and E01928 Test Sequences

Day-to-day variation was also determined for the 1992 Detroit Diesel DDC S60 with the TCEQ Tex LED Ref. fuel. The E02099 and the E02107 test sequences were 
used for this, and they were conducted two days apart. Three repeat tests were recorded for each sequence. Figure 37 displays the total COV for all six tests, which was the worst day-to-day variation for this research. Only a small range of particle sizes, $55 \mathrm{~nm}$ to 205 $\mathrm{nm}$, had a COV of less than $5 \%$. The fuel used in these six tests caused greater run-torun variation than most other fuels that were tested with the DDC S60; meaning that a change in fuel properties was responsible for increased day-to-day variation.

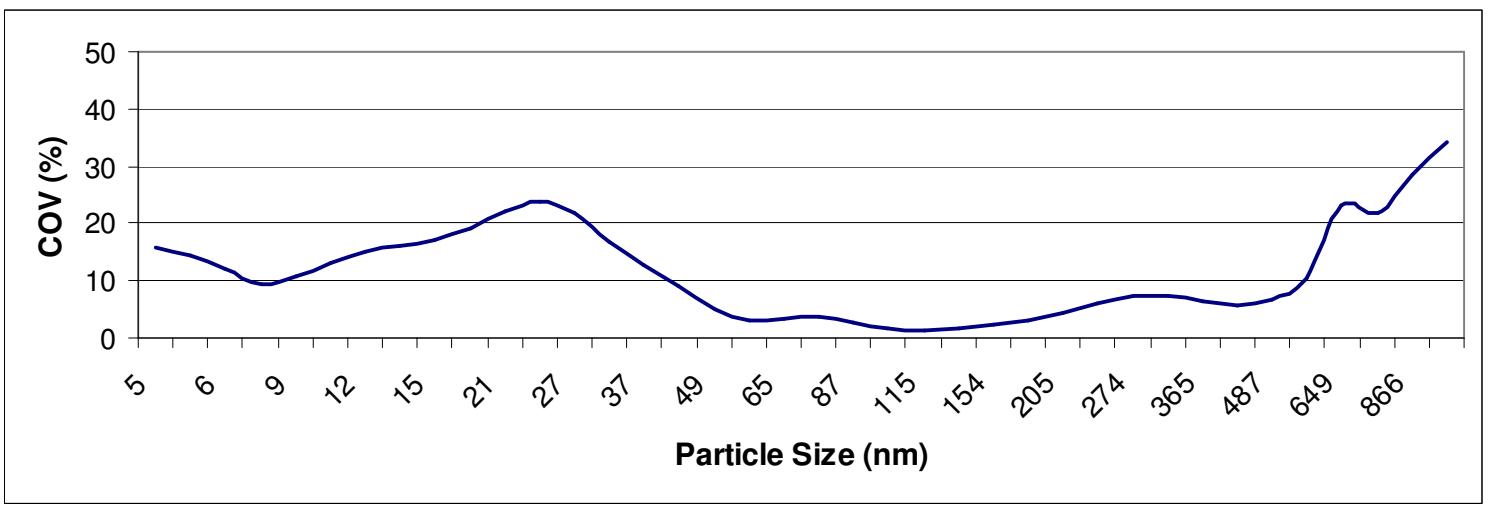

Figure 37 Day-to-Day Variation of the 1992 DDC S60

Percent differences between the E02099 sequence and the E02107 test sequence can be observed in Figure 38. In Figure 38, the darker columns represent particle sizes where significant day-to-day variation occurred. Similarly to the differences in Figure 36, day-to-day differences in this case are significant between $32 \mathrm{~nm}$ and $487 \mathrm{~nm}$. In the entirety of this research, the largest significant difference from the results of two separate days occurred with this fuel and engine, which was about $26 \%$ at $32 \mathrm{~nm}$. 


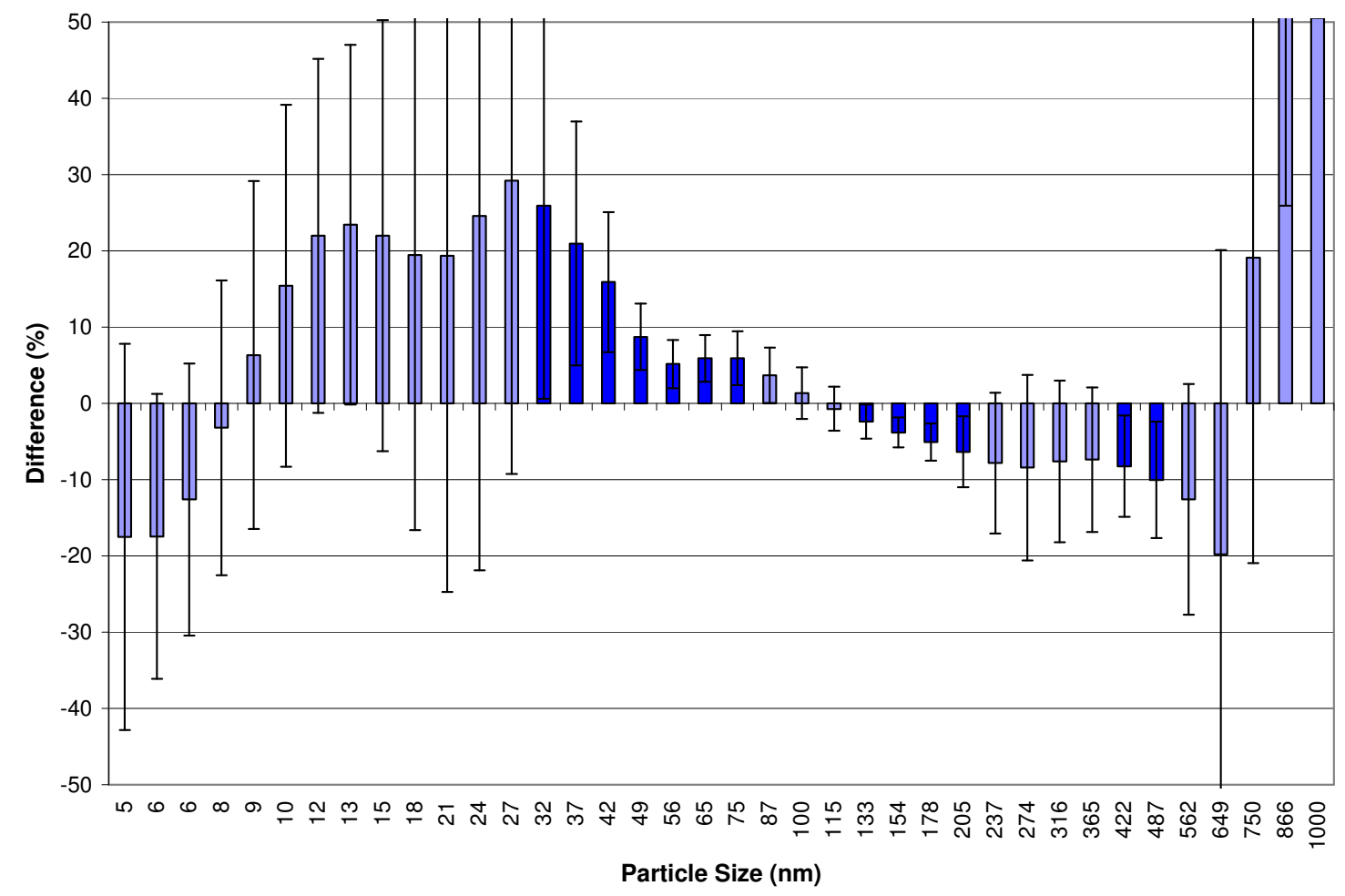

Figure 38 Particle Concentration Differences between E02099 and E02107 Test Sequences

Error bars on most of the dark blue columns in Figures 36 and 38 nearly cross the $\mathrm{x}$-axis, which means that the day-to-day differences in these circumstances may not have been significant if more tests had been run on each day. It is also possible that the significant differences were caused by changes in barometric pressure. Barometric pressure had average values of 14.155 psi during testing the E01927 test sequence, 14.330 during the E01928 test sequence, 14.153 psi during the E02099 test sequence, and 14.243 psi during the E02107 test sequence. As discussed in the Extremes of Run-to-Run Variation section, the ISM 370 and the DDC S60 produced much greater test-to-test variability than the rebuilt DDC S60. Day-to-day variation results are not presented for the rebuilt DDC S60, because there were not enough repeat tests of any fuel from 
separate days. Day-to-day variation hindered the certainty of results in the following sections, and it was an issue that was discussed in some of the conclusions.

\subsubsection{Base Fuels vs. Additized Fuels}

Test sequences E02136, E02138, and E02140 each consisted of three hot-start FTPs, and they were performed on the same day with the rebuilt DDC S60 engine. E02136 was run on low cetane, E02138 was run on low cetane B10A, and E02140 was run on low cetane B20A. Low cetane B10A was a mixture of $90 \%$ low cetane diesel and $10 \%$ biodiesel by mass, and low cetane B20A was $80 \%$ low cetane and $20 \%$ biodiesel. Figure 39 compares the average particle concentrations of low cetane and low cetane B10A. There appear to be significant differences from $6 \mathrm{~nm}$ to $56 \mathrm{~nm}$ and $100 \mathrm{~nm}$ to 487 $\mathrm{nm}$, because the standard deviation lines of E02136 and E02138 do not overlap in those ranges. Nuclei mode and accumulation mode can clearly be distinguished here, unlike with the "straight line" pattern produced with the ISM 370. In both cases below, the nuclei mode peak is about $4 * 10^{13}$ particles/bhp-hr at $21 \mathrm{~nm}$, and the accumulation mode peak is close to $2 * 10^{13}$ particles/bhp-hr at $140 \mathrm{~nm}$. 


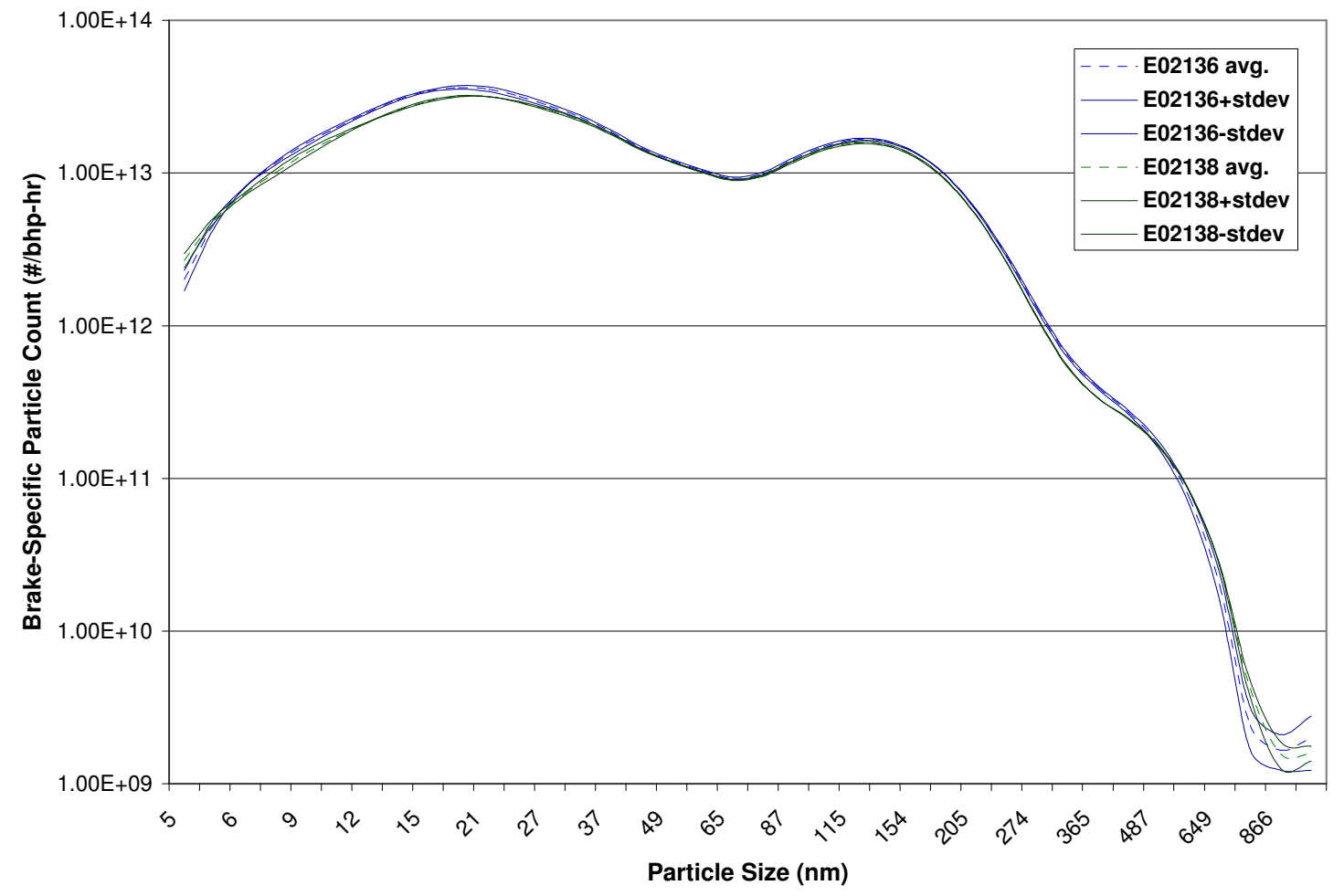

Figure 39 Particle Concentration Comparison of E02136 and E02138 Test Sequences

A comparison of the particle distributions from low cetane and low cetane B20A is represented by Figure 40. The ranges of particle diameters where significant differences can be seen are the same in Figure 39 as they are in Figure 40. It is clear that the mixing of biodiesel with low cetane caused a decrease in particle concentrations over these diameters. 


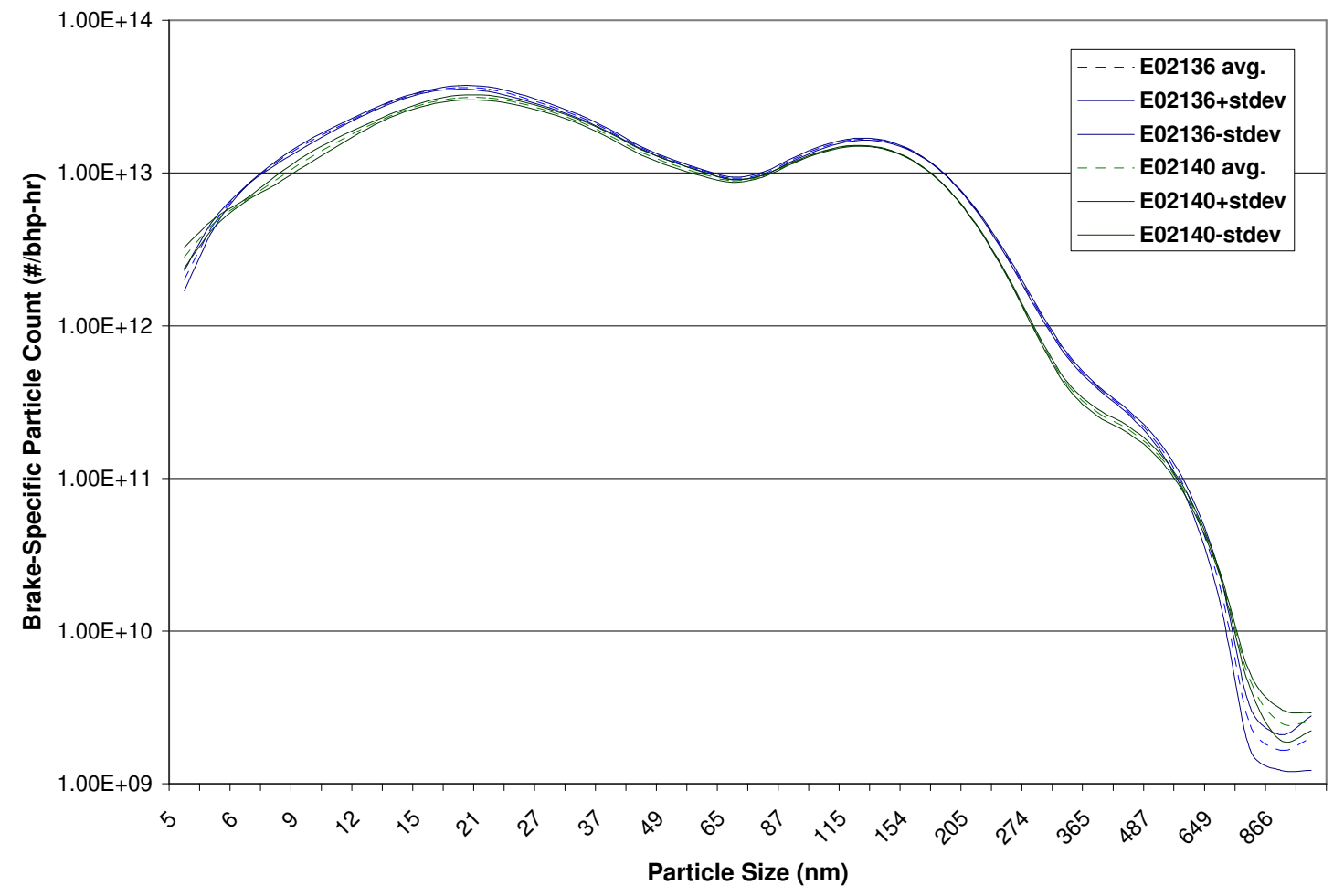

Figure 40 Particle Concentration Comparison of E02136 and E02140 Test Sequences

From this point on, error bars for the percent differences between particle distributions were calculated using the following equation.

$$
e=100 *\left(\frac{e_{1}+e_{2}}{a_{1}}\right)
$$

\section{Equation 11}

The letter $e$ stands for the magnitude of the error bar, $e_{1}$ represents the standard deviation of the base fuel testing (or original testing), $e_{2}$ represents the standard deviation of the additive testing (or comparative testing), and $a_{1}$ corresponds to the average value of the base fuel (or original) testing.

Differences between the average particle distributions of E02136, E02138, and E02140 are presented on a percent basis in Figure 41. The orange and yellow columns show differences between low cetane and low cetane B10A, where the orange columns 
represent significant differences and the yellow columns do not. The light and dark blue columns indicate differences between low cetane and low cetane B20A, where the dark blue columns represent significant differences. Using a $20 \%$ biodiesel blend magnified the effect of using a $10 \%$ blend. They both caused a decrease in average particle concentrations from about $6 \mathrm{~nm}$ to $49 \mathrm{~nm}$ and $100 \mathrm{~nm}$ to $487 \mathrm{~nm}$. The $10 \%$ biodiesel blend, or additive 1 , caused a $12 \%$ reduction of $8 \mathrm{~nm}$ particles and a $17 \%$ reduction of $316 \mathrm{~nm}$ particles. The $20 \%$ blend, or additive 2, caused a $23 \%$ reduction of $8 \mathrm{~nm}$ particles and a $36 \%$ reduction of $316 \mathrm{~nm}$ particles. For the most part, the reduction of particle concentrations due to mixing of biodiesel with low cetane is proportional to the amount of biodiesel in the fuel.

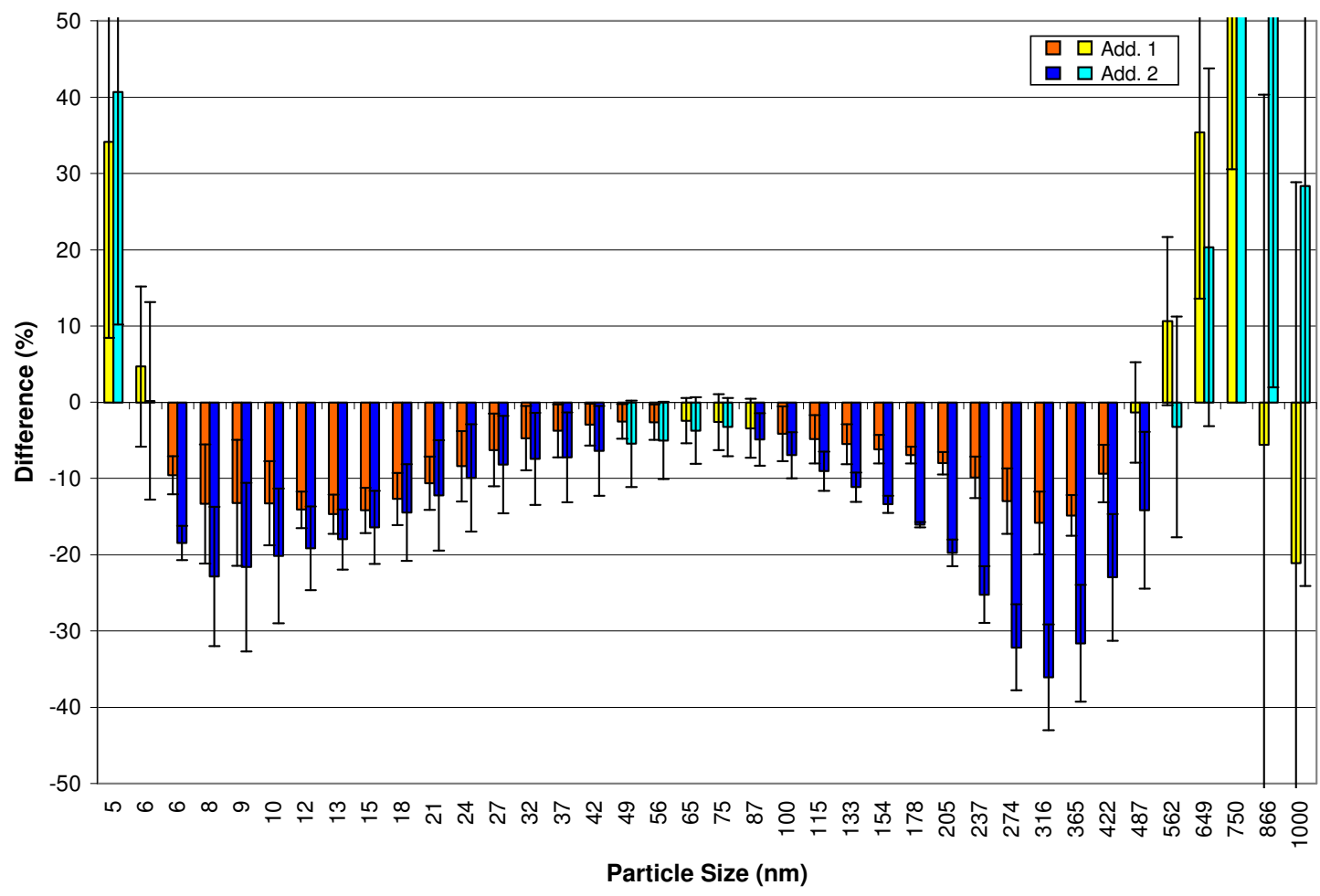

Figure 41 Differences in Particle Concentrations Due to Mixing of Biodiesel with Low Cetane Diesel Fuel 
The following comparison between fuels was done using the rebuilt DDC S60 and three fuel types. FEM trt. 3 corresponds to the test sequence E02195, FEM trt. 6 corresponds to E02198, and FEM trt. 7 corresponds to E02199. Each test sequence consisted of three hot-start FTPs, but the three sequences were not performed on the same day. Figure 42 illustrates the particle concentrations of FEM trt. 3 and FEM trt. 6 on a brake-specific basis. It is clear that the additive in FEM trt. 3 extensively differed in effect from the additive in FEM trt. 6. The nuclei mode from E02195 peaks about an order of magnitude higher that the nuclei mode from E02198, but the accumulation mode peaks lower. The additive in FEM trt. 6 demonstrated the greatest effect on particle concentration of any additive tested during this research.

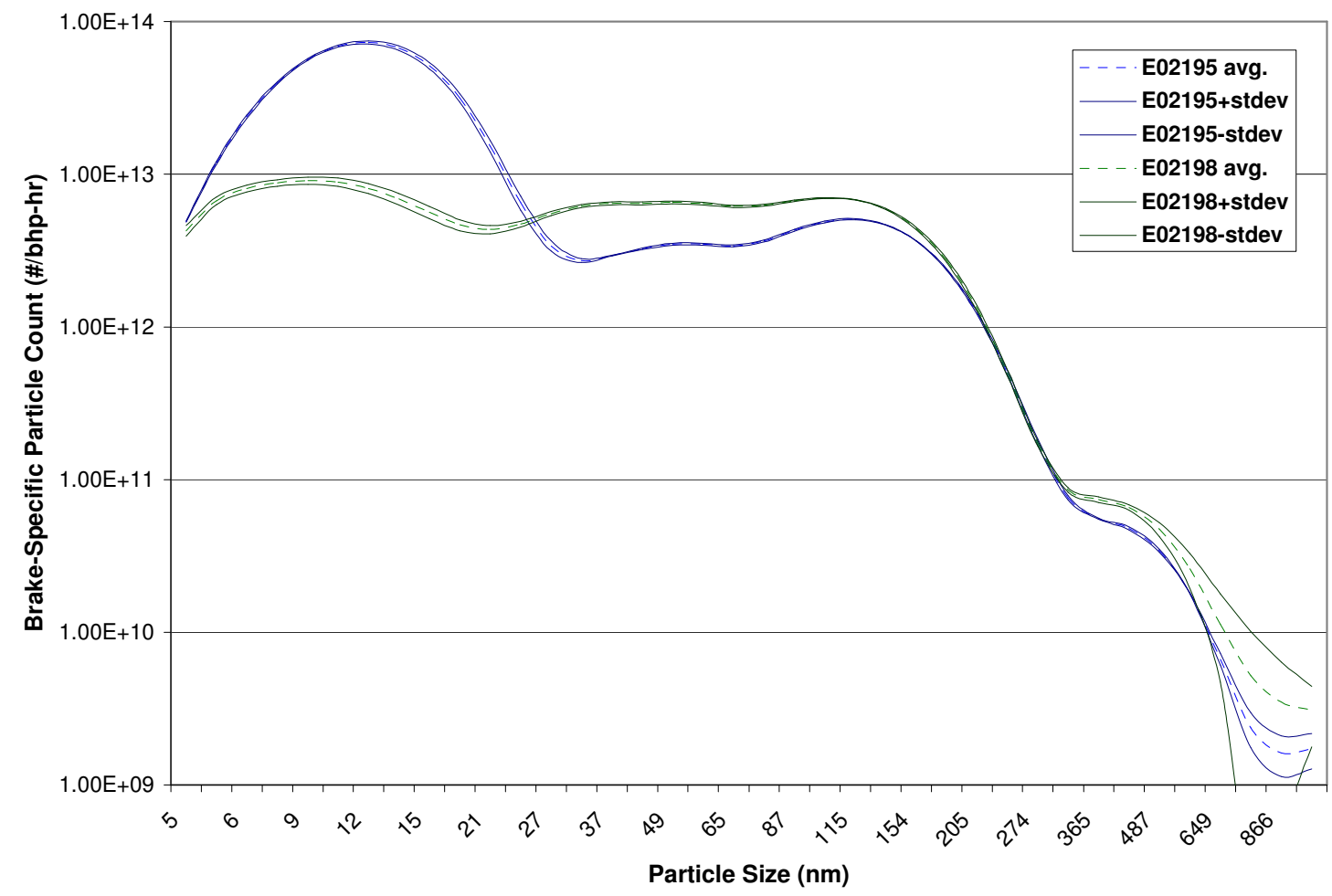

Figure 42 Particle Concentration Comparison of E02195 and E02198 Test Sequences 
Below, in Figure 43, a comparison between the particle size distributions of FEM trt. 3 and FEM trt. 7 is presented. The trend lines are similar in shape, which suggests that FEM trt. 7 consisted of a different amount of the same additive in FEM trt. 3. There were significant differences between FEM trt. 3 and FEM trt. 7, but it is difficult to determine by visual inspection of Figure 43.

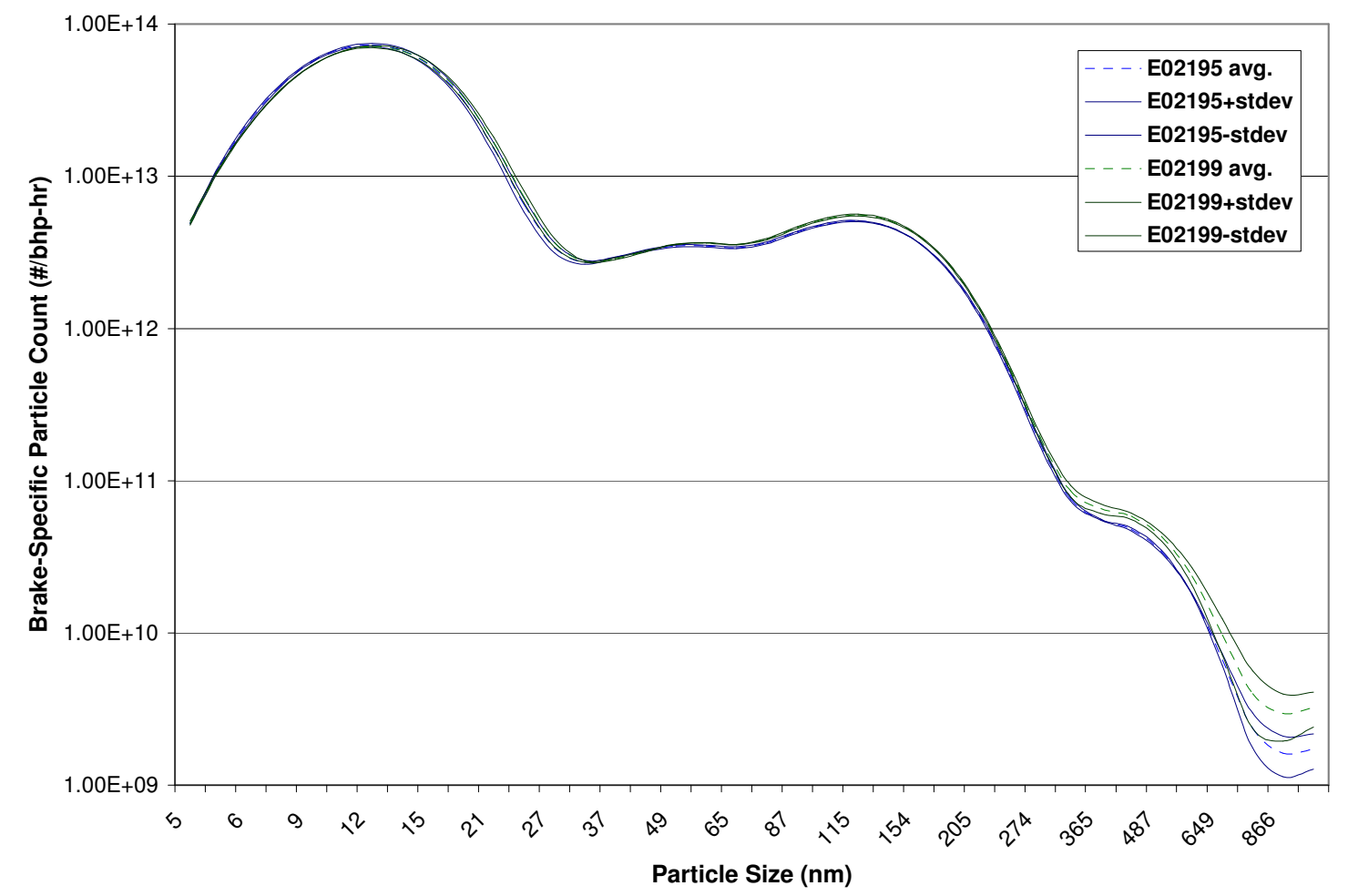

\section{Figure 43 Particle Concentration Comparison of E02195 and E02199 Test Sequences}

Significant differences between the average particle distributions of FEM trt. 3, FEM trt. 6 (additive 1), and FEM trt. 7 (additive 2) were determined on a percent basis and are illustrated in Figure 44. The orange columns represent the significant differences caused by the additives in FEM trt. 6, and the dark blue columns represent the significant differences due to the addition of FEM trt. 7. Considering FEM trt. 3 as the base fuel, FEM trt. 6 displayed a $15 \%$ to $88 \%$ decrease in nuclei mode particles between $5 \mathrm{~nm}$ and 
$24 \mathrm{~nm}$, a $62 \%$ to $130 \%$ increase in nuclei mode particles between $27 \mathrm{~nm}$ and $40 \mathrm{~nm}$, and a $10 \%$ to $98 \%$ increase in accumulation mode particles. FEM trt. 7 had a milder effect, in that its use resulted in a $3 \%$ to $8 \%$ decrease in nuclei mode particles and a $3 \%$ to 32 $\%$ increase in accumulation mode particles. It is probably safe to conclude that there are completely different additives in the fuels named FEM trt. 6 and FEM trt. 7. It is also apparent that fuel properties are a determining factor for the ranges and concentrations of the nuclei and accumulation modes.

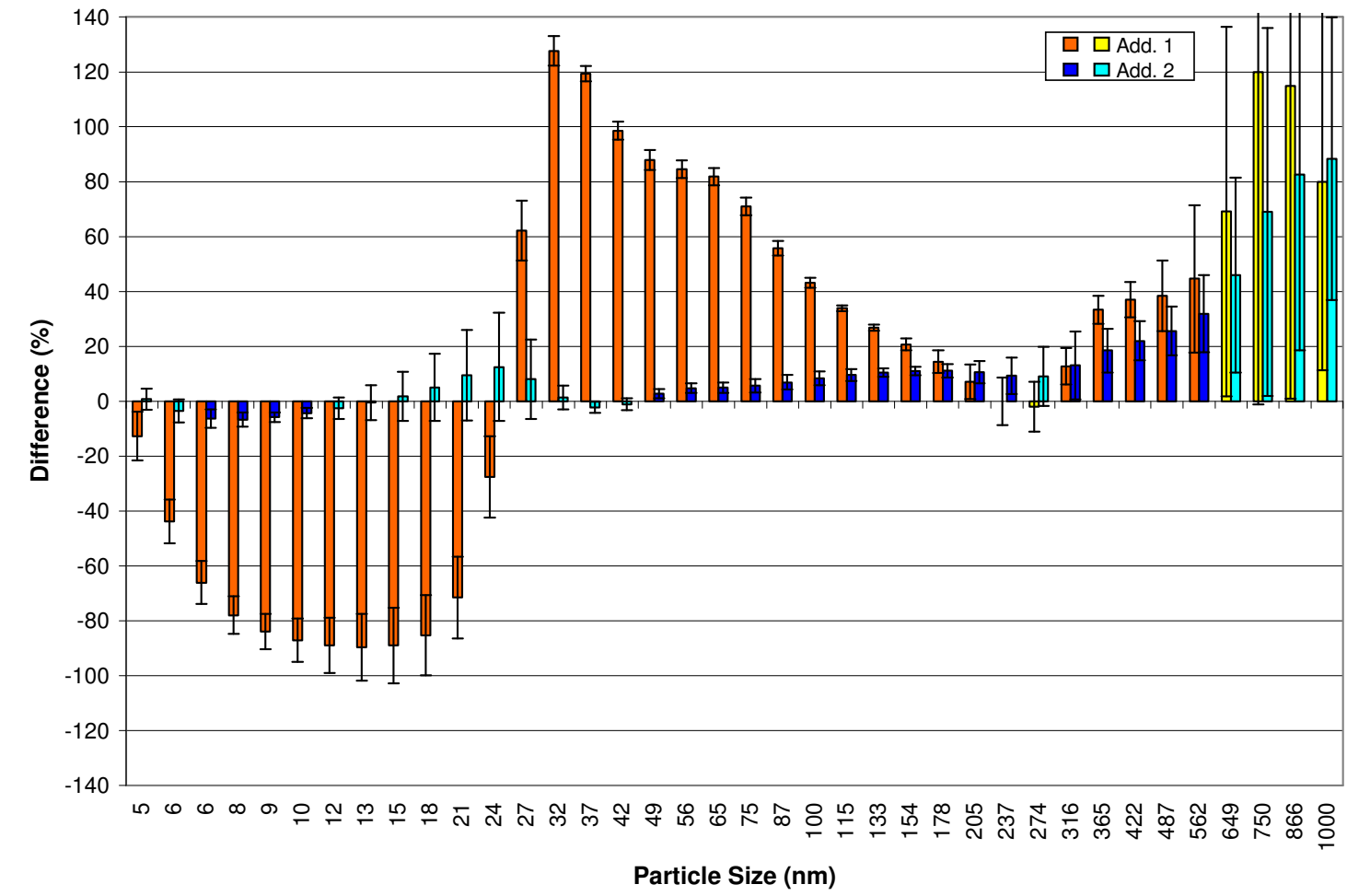

Figure 44 Differences in Particle Concentrations Due to Mixing of Fuel Additives with FEM Diesel Fuel

Another base fuel versus additive comparison was done on the rebuilt DDC S60 with low cetane as the base fuel and trt. 2-10 as the nine additives. Trt. 2 corresponds to additive 1, trt. 3 to additive 2, and so on. Testing took place on four consecutive days, and each fuel was tested over three hot-start FTPs. As can be seen in Table A-1 in the 
Appendix, test sequence E01943 corresponds to low cetane, and the test sequences between E01946 and E01964 that end in odd numbers correspond to additives 2 through 9, respectively. Fuel properties of the low cetane fuel are provided below in Table 9.

Table 9 Low Cetane Diesel Fuel Properties

\begin{tabular}{|c|c|c|c|}
\hline Fuel Property & ASTM Test Method & Unit of Measurement & Measurement \\
\hline Specific Gravity 60/60 & ASTM D 4052 & - & 0.8598 \\
\hline API Gravity & ASTM D 1250 & - & 33.1 \\
\hline Cloud Point & ASTM D 2500 & ${ }^{\circ} \mathrm{F}$ & -20 \\
\hline Flash Point, PM & ASTM D 93 & ${ }^{\circ} \mathrm{F}$ & 151 \\
\hline Sulfur & ASTM D 5453 & ppm & 8 \\
\hline Viscosity @ 40ㄷ & ASTM D 445 & cSt & 2.67 \\
\hline Hydrogen & ASTM D 3343 & Wt\% & 12.56 \\
\hline Carbon & ASTM D 5291 & $\mathrm{Wt} \%$ & 85.23 \\
\hline Cetane Number & ASTM D 613 & - & 46.2 \\
\hline Cetane Index & ASTM D 976 & - & 43.4 \\
\hline Distillation - IBP & \multirow{16}{*}{ ASTM D 86} & ${ }^{\circ} \mathrm{F}$ & 354.1 \\
\hline Distillation - 5\% & & ${ }^{\circ} \mathrm{F}$ & 381.2 \\
\hline Distillation - $10 \%$ & & ${ }^{\circ} \mathrm{F}$ & 414.2 \\
\hline Distillation - $20 \%$ & & ${ }^{\circ} \mathrm{F}$ & 441.8 \\
\hline Distillation - $30 \%$ & & ${ }^{\circ} \mathrm{F}$ & 463.5 \\
\hline Distillation - 40\% & & ${ }^{\circ} \mathrm{F}$ & 482.8 \\
\hline Distillation - 50\% & & ${ }^{\circ} \mathrm{F}$ & 500.8 \\
\hline Distillation - 60\% & & ${ }^{\circ} \mathrm{F}$ & 520.8 \\
\hline Distillation - 70\% & & ${ }^{\circ} \mathrm{F}$ & 542 \\
\hline Distillation - $80 \%$ & & ${ }^{\circ} \mathrm{F}$ & 565.5 \\
\hline Distillation - 90\% & & ${ }^{\circ} \mathrm{F}$ & 595.2 \\
\hline Distillation - 95\% & & ${ }^{\circ} \mathrm{F}$ & 619.3 \\
\hline Distillation - EP & & ${ }^{\circ} \mathrm{F}$ & 641.7 \\
\hline Distillation - Loss & & $\mathrm{ml}$ & 2.1 \\
\hline Distillation - Residue & & $\mathrm{ml}$ & 1 \\
\hline Distillation - Recovered & & $\mathrm{ml}$ & 96.9 \\
\hline
\end{tabular}

The low cetane fuel essentially had the same properties as the nine versions, LC trt. 2-10, where additives were present. The only practical differences were their cetane numbers, which are shown in Table 10. Also shown in Table 10 are the types and amounts of each additive. 
Table 10 Cetane Numbers and Additive Amounts of Low Cetane Fuels

\begin{tabular}{|c|c|c|c|c|c|c|c|c|c|}
\hline Low Cetane & LC trt. 2 & LC trt. 3 & LC trt. 4 & LC trt. 5 & LC trt. 6 & LC trt. 7 & LC trt. 8 & LC trt. 9 & LC trt. 10 \\
\hline 46.2 & 46.7 & 49.0 & 49.9 & 46.3 & 49.6 & 49.2 & 47.8 & 48.5 & 49.0 \\
\hline N/A & $\begin{array}{c}3-\mathrm{ml} \\
2 \mathrm{EHN}\end{array}$ & $\begin{array}{c}6-\mathrm{ml} \\
2 \mathrm{EHN}\end{array}$ & $\begin{array}{c}8-\mathrm{ml} \\
2 \mathrm{EHN}\end{array}$ & $\begin{array}{c}6-\mathrm{ml} \\
\mathrm{P} 1\end{array}$ & $\begin{array}{c}12-\mathrm{ml} \\
\mathrm{P} 1\end{array}$ & $\begin{array}{c}16-\mathrm{ml} \\
\mathrm{P} 1\end{array}$ & $\begin{array}{c}6-\mathrm{ml} \\
\mathrm{P} 2\end{array}$ & $\begin{array}{c}12-\mathrm{ml} \\
\mathrm{P} 2\end{array}$ & $\begin{array}{c}18-\mathrm{ml} \\
\mathrm{P} 2\end{array}$ \\
\hline
\end{tabular}

The names of the additives in Table 10 named P1 and P2 are proprietary, and the amounts of each additive are per gallon of fuel. All nine low cetane fuels with additives had greater cetane numbers than the base fuel. The fuel with the highest cetane number of 49.9 was LC trt. 4 . The rest of the fuels had cetane numbers ranging from 46.2 to 49.6. Table 11 and Figure 45, on the following pages, indicate that as the amount of each additive increases, there is generally an increase in cetane number and a decrease in nuclei mode particles. The dark gray cells in Table 11 belong to differences that are not significant between the base fuel and its additive versions. Significant decreases in concentration are within light blue cells, and significant increases due to the additives are in light orange cells. 
Table 11 Differences in Average Particle Concentrations Due to Mixing of Additives with Low Cetane Diesel

\begin{tabular}{|c|c|c|c|c|c|c|c|c|c|c|c|c|c|c|c|c|}
\hline \multicolumn{3}{|c|}{ Particle Size (nm) } & 6.49 & \multicolumn{2}{|c|}{7.50} & 8.66 & 10.00 & 11.55 & 13.34 & 15.40 & 17.78 & 20.54 & 23.71 & 27.38 & 31.62 & 36.52 \\
\hline \multirow{9}{*}{\multicolumn{2}{|c|}{ Difference }} & Add. $1(\%)$ & \multicolumn{2}{|c|}{6.12} & 5.15 & \multicolumn{2}{|c|}{-0.65} & -7.01 & \begin{tabular}{l|l}
1.54 & -14.08 \\
\end{tabular} & -15.55 & \begin{tabular}{|l|l|}
5 & -16.63 \\
\end{tabular} & -17.47 & -18.03 & -17.98 & -17.44 & -16.61 \\
\hline & & Add. $2(\%)$ & \multicolumn{2}{|c|}{-0.75} & -4.63 & -7.81 & \multicolumn{2}{|c|}{\begin{tabular}{|l|}
-10.58 \\
\end{tabular}} & \begin{tabular}{l|l}
2.23 & -13.39 \\
\end{tabular} & -14.79 & \begin{tabular}{|l|l|}
9 & -16.52 \\
\end{tabular} & -18.19 & -19.36 & -19.60 & -18.94 & -17.45 \\
\hline & & Add. $3(\%)$ & \multicolumn{2}{|c|}{4.05} & -3.38 & -11.74 & -18.98 & -24.13 & -27.11 & -28.90 & -30.05 & -30.62 & 0.64 & 0.59 & -30.66 & -29.74 \\
\hline & & Add. $4(\%)$ & & 13 & -5.72 & -8 & -13 & \begin{tabular}{l|l}
3.51 & -17 \\
\end{tabular} & -19.87 & -22.35 & \begin{tabular}{|l|l|}
5 & -24.90 \\
\end{tabular} & -26.83 & -27.77 & -28.09 & -28.28 & -27.82 \\
\hline & & Add $5(\%$ & & 45 & 1.60 & -6 & -13 & \begin{tabular}{l|l}
3.30 & -18 \\
\end{tabular} & -23.07 & -26.29 & -28.95 & -30.83 & -31.79 & -32.24 & -32.44 & -31.61 \\
\hline & & Add. $6(\%$ & & 65 & -7.88 & -14 & 73 & \begin{tabular}{l|l|}
1.76 & -27 \\
\end{tabular} & \begin{tabular}{l|l}
.10 & -30.38 \\
\end{tabular} & -32.60 & -34.23 & -35.08 & -35.08 & -34.92 & -34.93 & -33.78 \\
\hline & & Add. 7 & -10 & & -9.72 & -12 & 85 & \begin{tabular}{l|l}
.01 & -20 \\
\end{tabular} & \begin{tabular}{l|l|}
0.20 & -21.81 \\
\end{tabular} & -22.61 & -23.23 & -23.92 & -24.60 & -25.50 & -26.48 & -26.36 \\
\hline & & Add. 8 & -10 & & 16.62 & -22 & -27 & \begin{tabular}{l|l}
.85 & -31 \\
\end{tabular} & \begin{tabular}{l|l}
1.42 & -33.32 \\
\end{tabular} & -34.46 & -35.38 & -36.20 & -36.86 & -37.75 & -38.69 & -38.13 \\
\hline & & Add. $9(\%$ & -15 & & 22.01 & -27 & \begin{tabular}{l|l}
39 & -3 \\
\end{tabular} & $\begin{array}{l}3.08 \\
.087 \\
\end{array}$ & \begin{tabular}{l|l|l|}
.19 & -39.31 \\
\end{tabular} & 1] -40.43 & -41.07 & \begin{tabular}{|l|}
-41.23 \\
\end{tabular} & -40.86 & -40.94 & -41.56 & -40.44 \\
\hline 17 & 70 & 56.23 & 4.94 & 4.99 & 86 & & & & 133.35 & 53.99 & 177.83 & 205.35 & 237.14 & 273.84 & 316.23 & \begin{tabular}{l|l}
3 & 365.17
\end{tabular} \\
\hline 4.45 & 014 & 1.79 & 1.51 & 07 & & 24 & 4.26 & 1.72 & -1.10 & -4.28 & -8.00 & -12.23 & -16.17 & -16.50 & -9.6 & \\
\hline 4.33 & & & 54 & 58 & & & 1.49 & -0.92 & -2.90 & -4.51 & -5.66 & -6.0 & -4.54 & 0.86 & 10.6 & \\
\hline-26.69 & & -13.44 & -3.28 & 14 & & 09 & -6.66 & -10.65 & -13.37 & 4.57 & -13.85 & -10.53 & -3.41 & 8.11 & 18.4 & 15.07 \\
\hline-25.43 & 0.11 & 12.89 & -4.45 & 0.5 & 3 & 53 & -3.72 & -7.11 & -10.35 & -13.51 & -16.62 & -19.35 & -20.36 & -16.09 & -4.3 & 2.41 \\
\hline-28.63 & 3.18 & 5.20 & -5.48 & -1.24 & & 12 & -8.27 & -11.80 & -14.10 & -14.99 & -14.08 & -10.47 & -2.45 & 11.43 & 24.8 & 21.60 \\
\hline-30.13 & & & -2.65 & 64 & -2 & 77 & 21 & -12 & 5.24 & -15.72 & -13.59 & -7.98 & 2.36 & 16.92 & 25.8 & 17.0 \\
\hline-23.96 & -18.98 & \begin{tabular}{l|l}
8 & -11.23 \\
\end{tabular} & -1.60 & 2.13 & -1 & 96 & -7.59 & -12.69 & -16.80 & -20.04 & -22.36 & -23.34 & -21.40 & -12.94 & 2.8 & 37 \\
\hline-34.71 & -28.38 & $\begin{array}{l}8 \\
\end{array}$ & -6.42 & -1.94 & -6 & 88 & -13.14 & -18.44 & -22.03 & -23.76 & -23.22 & -19.49 & -10.63 & 5.26 & 21.4 & 18.46 \\
\hline-36.09 & -28.38 & 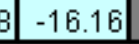 & -1.04 & 4.18 & & 16 & -9.90 & -16.25 & -20.30 & -21.69 & -19.93 & -14.09 & -2.74 & 13.61 & 23.3 & 12.3 \\
\hline
\end{tabular}


The method in which significant difference was determined was the same as in the previous base fuel versus additive cases. On a concentration plot, the standard deviation lines would overlap for the same particle sizes which have gray cells. The greatest decrease in concentration caused by the $2 \mathrm{EHN}$ additive was a $30.7 \%$ difference in $31.6 \mathrm{~nm}$ particles. In this case, $8 \mathrm{ml}$ of $2 \mathrm{EHN}$ was added; and there was also a $14.6 \%$ decrease in $154 \mathrm{~nm}$ particles and an $18.4 \%$ increase in $316 \mathrm{~nm}$ particles. The largest amount of P1 that was added was $16 \mathrm{ml}$. This amount led to the most significant results for the additive, where there was a $35.1 \%$ decrease in $20.5 \mathrm{~nm}$ particles, a $15.7 \%$ decrease in $154 \mathrm{~nm}$ particles, and a $25.9 \%$ increase in $316 \mathrm{~nm}$ particles. Similarly, P2 demonstrated its most prominent differences when $18 \mathrm{ml}$ was added. This resulted in the most significant overall reduction due to any of the nine additives. There was a $41.1 \%$ decrease in $20.5 \mathrm{~nm}$ particles, a $21.7 \%$ decrease in $154 \mathrm{~nm}$ particles, and a $23.3 \%$ increase in $316 \mathrm{~nm}$ particles. The percent differences discussed in this paragraph were the maximum differences in the nuclei, accumulation, and course modes due to the three types of additives. The largest particle diameter in Table 11 is 365.17 . This is because there were no significant differences beyond that size. Figure 45 illustrates all differences due to the various amounts of each additive. The differences in Figure 45 are based on average distributions for each fuel, and significance is not specified. It was noted that there was no continuum between additives 4 and 5, nor between additives 6 and 7; but the plot does show a continuum within the individual additive types (1-3, 4-6, and 7-9). 


\begin{tabular}{|llllllll}
\hline$\square-60.00--50.00$ & $\square-50.00--40.00$ & $\square-40.00--30.00$ & $\square-30.00--20.00$ & $\square-20.00--10.00$ & $\square-10.00-0.00$ & $\square 0.00-10.00$ & $\square 10.00-20.00$ \\
$\square 30.00-40.00$ & $\square 40.00-50.00$ & $\square 50.00-60.00$ & $\square 60.00-70.00$ & $\square 70.00-80.00$ & $\square 80.00-90.00$ & $\square 90.00-100.00$
\end{tabular}

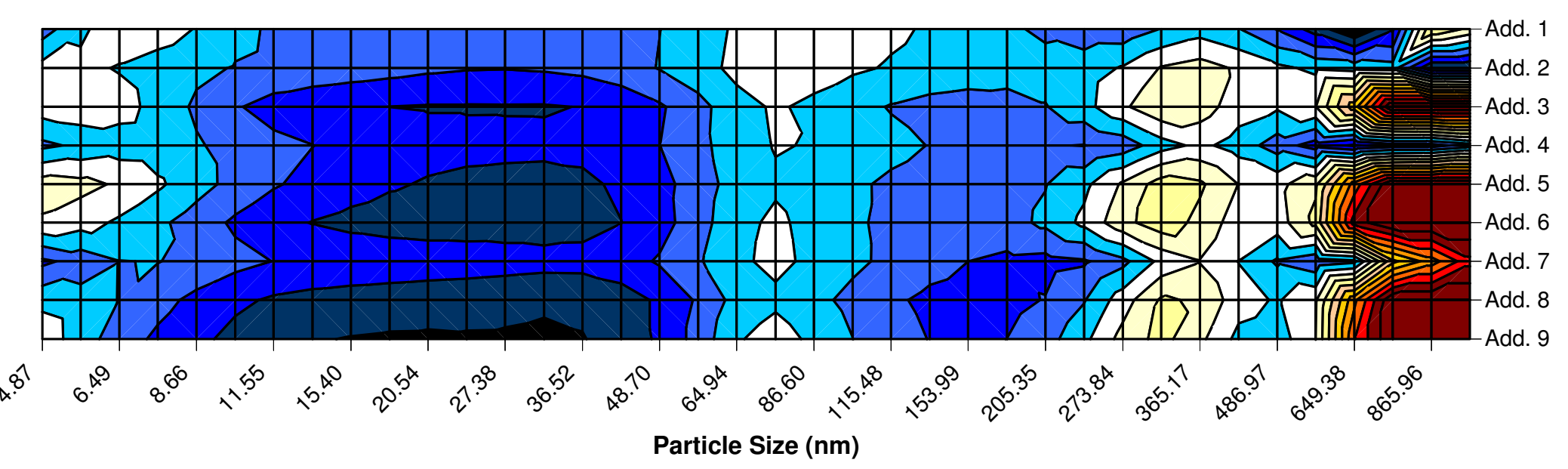

Figure 45 Percent Differences in Particle Distributions Due to Mixing of Additives with Low Cetane Diesel 
It can be deduced from inspection of Figure 45 that average particle distributions are more closely related to the amount of the additive rather than the cetane number. LC trt. 7 has a lower cetane number than LC trt. 6, but there was a greater reduction of particles due to LC trt. 7. This is the only case where there was a decrease in cetane number and a simultaneous increase in particle reduction. In all nine additive cases, an increase in the additive amount resulted in a reduction of nuclei mode and accumulation mode particles. The massive differences of greater than $90 \%$ in the particles larger than $650 \mathrm{~nm}$ were considered negligible because the COVs from each fuel were greater than $50 \%$. Day-to-day variation may have had an effect on these results, but it was impossible to determine because none of the fuels were tested on multiple days.

\subsubsection{Unheated Sample Line Effects}

The temperature of the sample line between the primary dilution tunnel and the secondary dilution system was varied by enabling and disabling the heating element. Test sequence E01967 consisted of two consecutive hot-start FTPs with an unheated sample line, and E01968 consisted of three consecutive hot-start FTPs with a heated sample line. The two sequences were completed on the same day with the DDC S60 and the 2007 CP Cert. diesel fuel. Figure 46 presents the COVs of each particle diameter over the E01967 test sequence. When the heating element was off, the average temperature at the exit end of the sample line was $32{ }^{\circ} \mathrm{C}$. E01967 was the only test sequence that resulted in COV values of less that $5 \%$ between $5 \mathrm{~nm}$ and $12 \mathrm{~nm}$. Since only 2 repeat tests were administered, the COV values were probably higher on average than if more tests had been performed. 


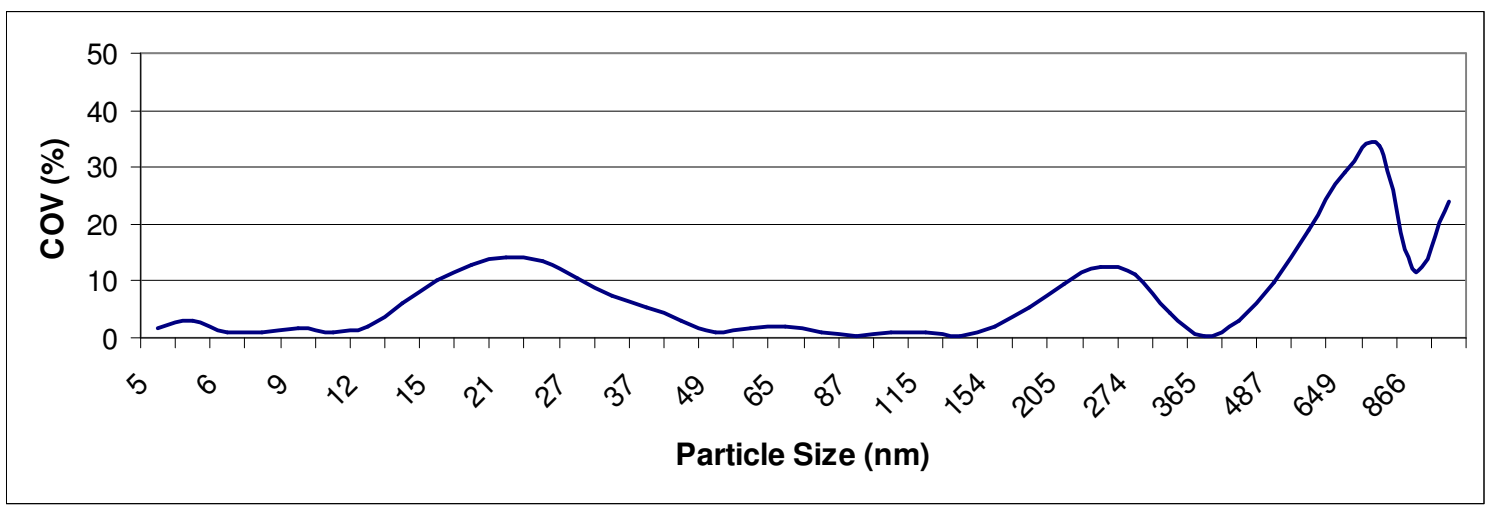

Figure 46 Run-to-Run Variation Using an Unheated Sample Line

The COV results for test sequence E01968, which were mostly lower than those of E01967, are shown below in Figure 47. During E01968, the temperature control box within the secondary dilution system maintained a temperature of $47+-1.0{ }^{\circ} \mathrm{C}$ at the exit end of the sample line. The particles between $5 \mathrm{~nm}$ and $15 \mathrm{~nm}$ had noticeably higher COVs when the sample line was heated.

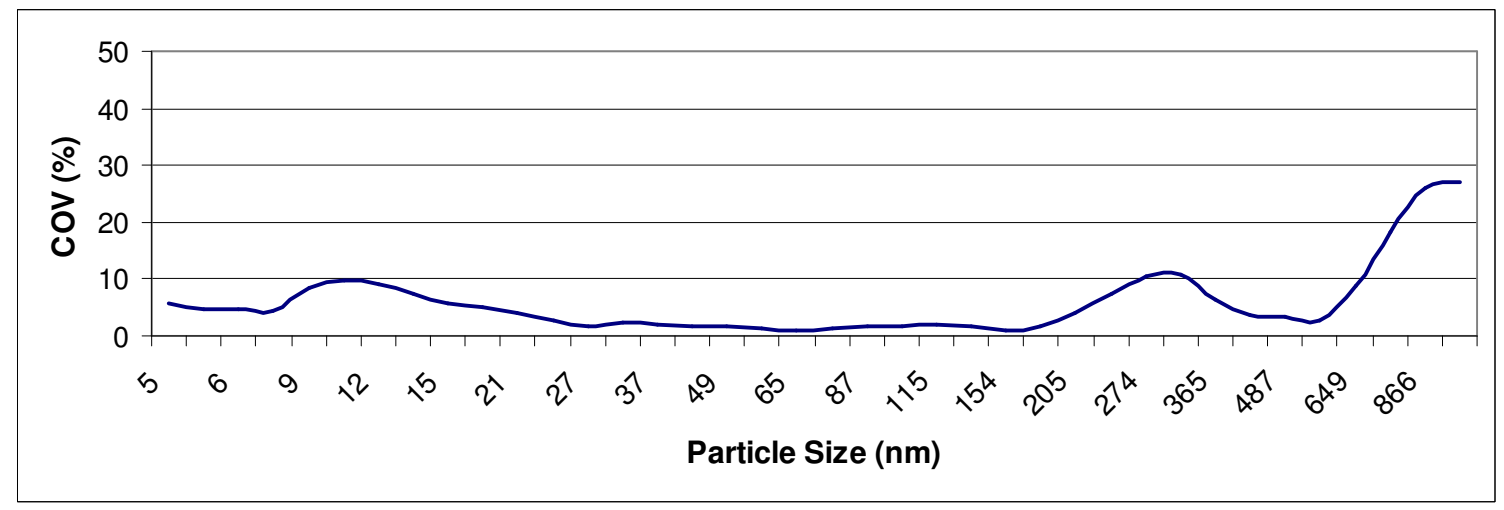

Figure 47 Run-to-Run Variation Using a Heated Sample Line

The concentrations of both test sequences can be observed in Figure 48. Disabling of the sample line heater resulted in a nearly identical concentration spectrum of nanoparticles. 


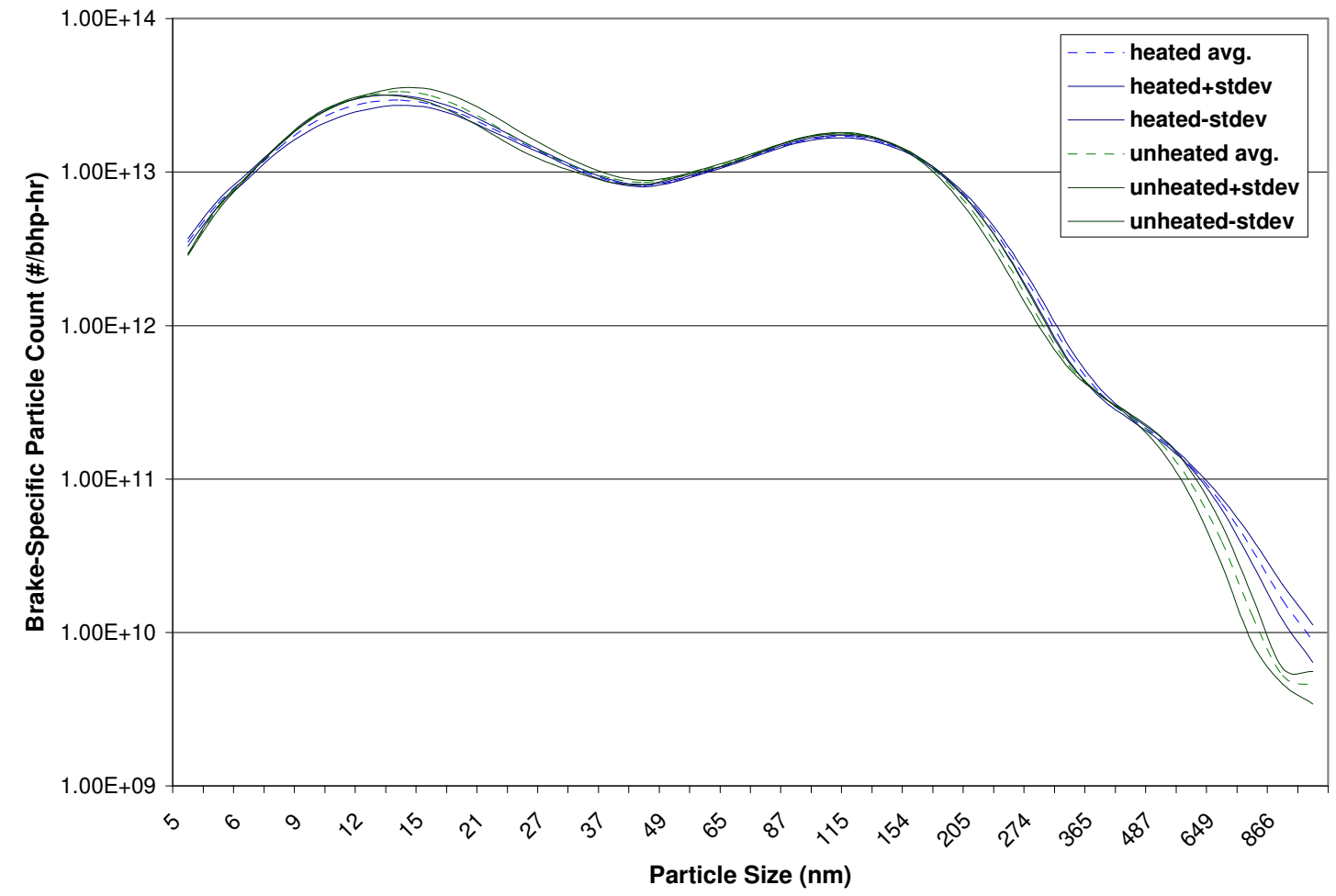
Figure 48 Particle Concentration Comparison of
E01967 and E01968 Test Sequences

Numerical differences caused by sampling with an unheated line appear in Figure 49, where the dark blue columns represent differences that were calculated to be significant. Excluding the $5 \mathrm{~nm}$ column, error bars span at least $50 \%$ of all of the dark blue columns' heights. This means that most of the discrepancies that are shown by dark blue columns probably should not be considered significant. The $5 \mathrm{~nm}$ particles, in which there was a $15 \%$ decrease in concentration, were the only ones that were considerably affected by the temperature of the sample line. 


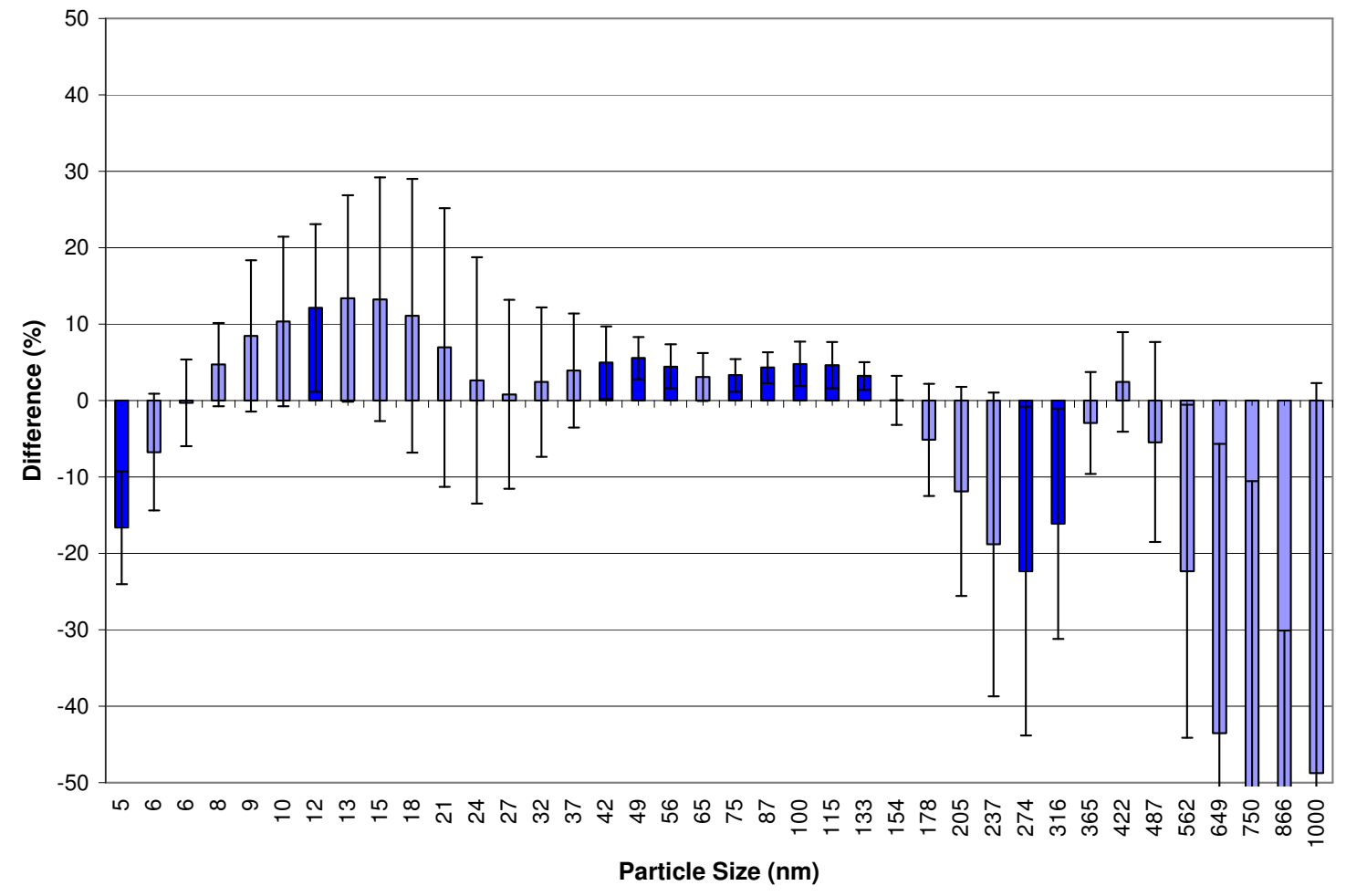

Figure 49 Particle Concentration Differences between Unheated and Heated Sample Line Results

In the existing literature, it is commonly noted that the temperature within the dilution environment is related to the formation of nanoparticles. The conclusion of this study was not the same, probably because the temperature of the DMS sample line (between the secondary dilution system and the DMS) always equalized with the temperature of the ambient air. Particles most likely finished mutating in the DMS sample line, where the final sample temperature always stabilized at around $22{ }^{\circ} \mathrm{C}$. The average significant difference over the particle spectrum was less than $1 \%$; which suggests that the average amounts of PM produced (on a mass basis) were within a few percent. In fact, the average amount of PM emitted during the E01967 test sequence was $0.262 \mathrm{~g} / \mathrm{bhp}-\mathrm{hr}$, and the average amount emitted over the E01968 sequence was 0.267 
g/bhp-hr. The mass-based amounts of PM were measured using gravimetric filters, and they differed by $1.87 \%$.

\subsubsection{Extremes of Run-to-Run Variation}

Run-to-run COV curves and particle concentration values were unique for each fuel. Test engines were also a factor in the $\mathrm{COV}$ and concentration results. A "COV score" was formulated as a means to numerically compare run-to-run variation between test conditions. A COV score of zero represents perfect run-to-run repeatability of PM concentrations between $5 \mathrm{~nm}$ and $1000 \mathrm{~nm}$. The COV score can be calculated using equation 12 below.

$$
\begin{aligned}
& \text { COVscore }=0.25 * \operatorname{COV}_{5-9}+0.50 * \operatorname{COV}_{10-19}+ \\
& \operatorname{COV}_{20-249}+0.50 * \operatorname{COV}_{250-499}+0.25 * \operatorname{COV}_{500-1000}
\end{aligned}
$$

\section{Equation 12}

The COV score, essentially, is a weighted sum of the COVs from each particle size. $\mathrm{COV}_{5-9}$ is the summation of all $\mathrm{COV}$ values between $5 \mathrm{~nm}$ and $9 \mathrm{~nm}$. Likewise, $C O V_{10-19}$ is the summation of COVs between $10 \mathrm{~nm}$ and $20 \mathrm{~nm}$, and so on. The constants $0.25,0.50$, and 1.0 are weighting factors that reduce biases due to excessively high variation near the upper and lower bounds of the measured particle spectrum. Note that the weighting factors in equation 12 sum to 2.0. The COV score would be equally as effective if the factors were divided by two, so that they would sum to 1.0. A bias, for instance, might exist between $200 \mathrm{~nm}$ and $1000 \mathrm{~nm}$ because particle concentrations in that size range were continually near or below the lower limit of detection (which is about $1000(\mathrm{dN} / \mathrm{d} \operatorname{logdp}) / \mathrm{cc})$. Another bias generally exists between $5 \mathrm{~nm}$ and $30 \mathrm{~nm}$. Causes of the biases, or variations within these ranges, are discussed in depth in the Continuous FTP Data section. 
Figure 50 displays the run-to-run variations of two test sequences performed using the ISM 370. E01928 was conducted with the NBB-B05 biodiesel blend. It consisted of three repeat hot-start FTPs, and it was the sequence that had the lowest COV score, which was 107 , for the Cummins engine. The COV score of the two hot-start FTPs in sequence E01921 was 321, which was the highest COV score found from all of the research done for this thesis. The parabolic shape of the E01928 and E01921 COV trend lines was apparent in all of the test sequences executed with the ISM 370. This might be attributed to the use of EGR and/or inconsistency in engine control by the ECU.

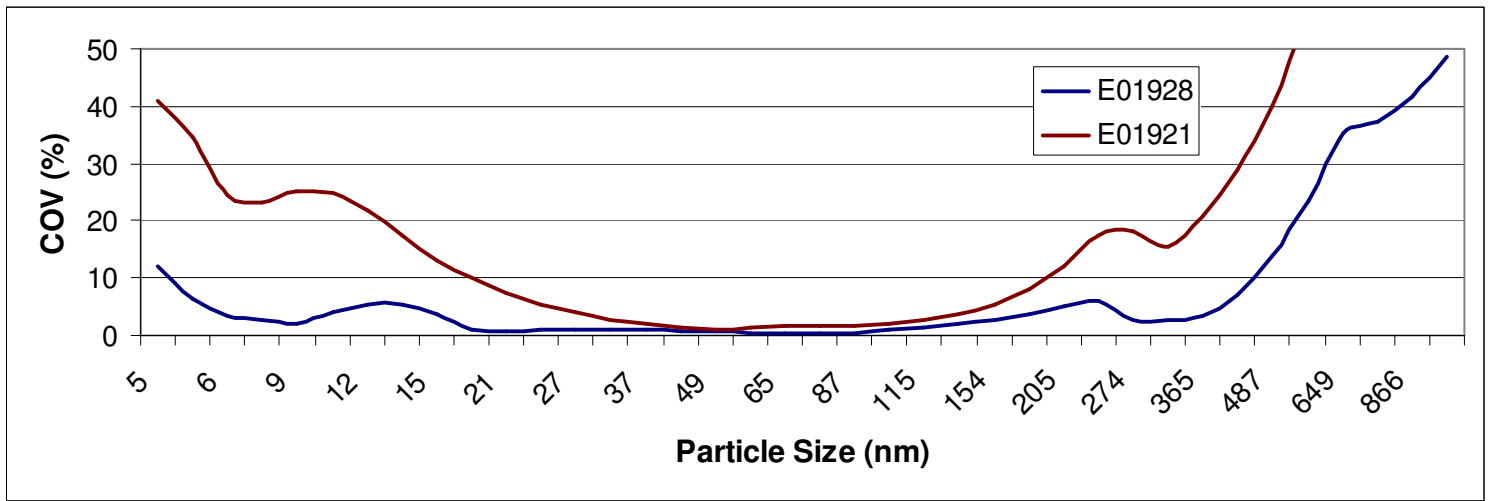

Figure 50 Run-to-Run Variations of Test Sequences E01928 and E01921

Test sequence E01977, which consisted of three consecutive hot-start FTPs, was performed with RF 71 diesel fuel on the DDC S60. E02099 also consisted of three hotstart FTPs, and it was performed using the DDC S60 and TCEQ Tex. LED Ref. diesel. As illustrated in Figure 51, the COV curves from E01977 and E02099, which accounted for the highest and lowest COV scores from the DDC S60, were similar in shape. Just as every COV trend from the ISM 370 displayed similar profiles; all of the COV plots from sequences tested on the DDC S60 were alike. They each had COV percentage values that prominently peaked around $27 \mathrm{~nm}$, were below $5 \%$ between $50 \mathrm{~nm}$ and $205 \mathrm{~nm}$, and peaked again at about $850 \mathrm{~nm}$. Test sequence E01977 produced a COV score of 117, and 
E02099 gave a COV score of 246. Various fuels were tested on the DDC S60, and the run-to-run variations were always similar; meaning that the "double ramp" shape in Figure 51 was probably caused by engine control issues.

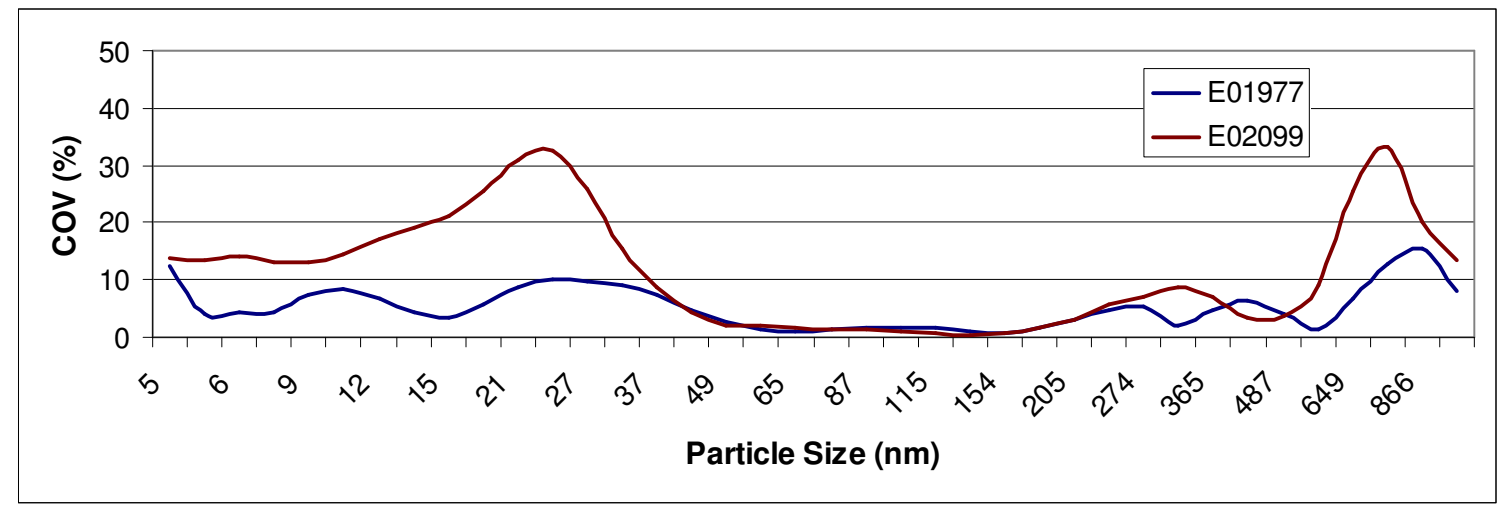

Figure 51 Run-to-Run Variations of Test Sequences E01977 and E02099

The COV curves with the highest and lowest COV scores of the test sequences performed on the rebuilt DDC S60 are shown in Figure 52. Three repeat hot-start FTPs were included in the E02138 test sequence. Low cetane B10A diesel fuel was used in E02138, and low cetane trt. 8 corresponds to E01959. According to a COV score of 41, the tests administered in E02138 boasted more precise particle distributions than any other tests. The COV line of E02138 peaked at $20 \%$ around $866 \mathrm{~nm}$; and it was less than $5 \%$ in the $6 \mathrm{~nm}$ to $750 \mathrm{~nm}$ diameter range and below $3 \%$ from $12 \mathrm{~nm}$ to $700 \mathrm{~nm}$. E01959, the test sequence with the greatest run-to-run variation from the rebuilt DDC S60, produced a COV score of 156. The shapes of the COV curves in Figure 52 are the same in that they are both within a $5 \%$ range between $6 \mathrm{~nm}$ and $487 \mathrm{~nm}$, and they each peak at about $866 \mathrm{~nm}$. This trend was evident in all of the test sequences completed with the rebuilt DDC S60. The DDC S60 and the rebuilt DDC S60 are of the same make and model, but they each encompass a distinct COV profile. This fact implies that there were dissimilarities in engine operation, which could include: control algorithms of the engine 
control unit (ECU), levels of engine wear, ages of fuel injectors, and manufacturing imperfections.

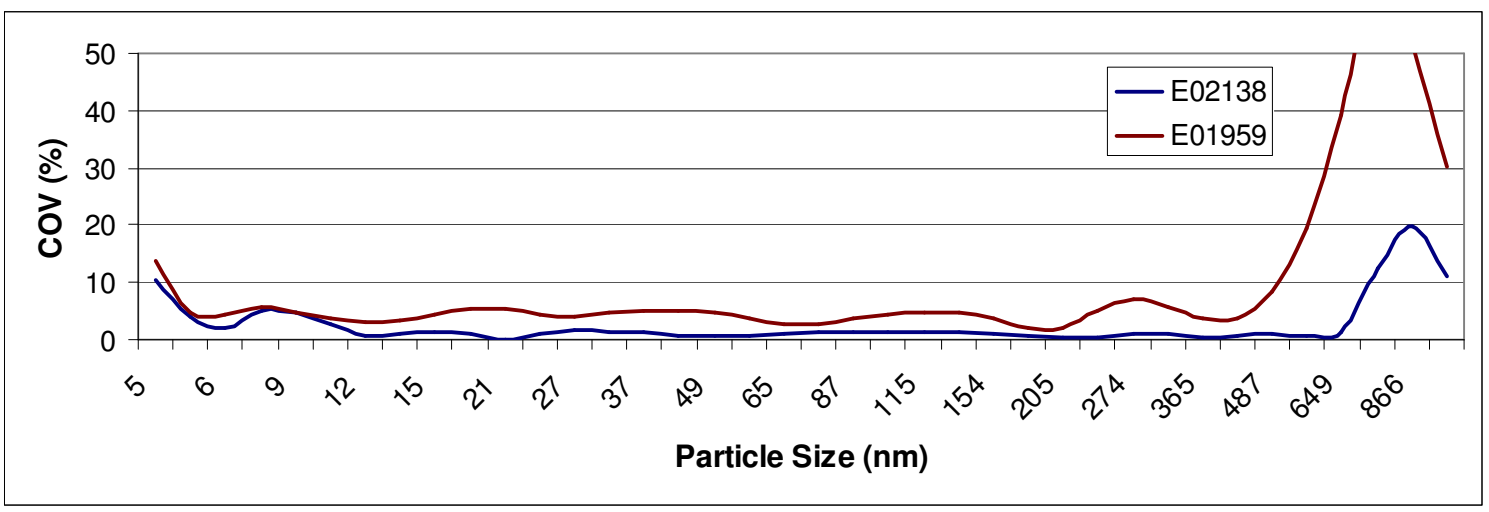

Figure 52 Run-to-Run Variations of Test Sequences E02138 and E01959

This research resulted in three basic run-to-run variation profiles. The "parabolic" profile of Figure 50 was specific to the ISM 370. The "double ramp" profile was generic for the DDC S60, and the mostly flat profile was common to the rebuilt DDC S60. Engine type apparently determines the general trend of run-to-run variation, but the magnitudes and COV scores of run-to-run variation are related to fuel properties. The average COV score of the tests completed with the rebuilt DDC S60 was 102. All COV scores from the rebuilt DDC S60 were at least 5 points lower that those of the ISM 370 and 15 points lower than those from the other DDC S60. The rebuilt DDC S60 commonly provided more repeatable results, which signifies that only a portion of the variations were caused by laboratory issues. Differences in engine and fuel properties were probably responsible for the remainder. Since 22 test sequences were completed on the rebuilt DDC S60 and none of them belonged to a COV score of higher than 156 points, it can be deduced that no more than 156 points of the COV scores from any of the test sequences during this study were due to laboratory inconsistencies. It is estimated that laboratory issues were generally responsible for $50 \mathrm{COV}$ score points or less. 
The average particle concentrations and standard deviations of test sequence E02138 are presented below in Figure 53. Again, E02138 was performed on the rebuilt DDC S60 with low cetane B10A, and it was the test sequence that demonstrated the least run-to-run variation. The standard deviation lines of Figure 53 are only distinguishable from the "average" line in the $5 \mathrm{~nm}$ to $6 \mathrm{~nm}$ and $700 \mathrm{~nm}$ to $1000 \mathrm{~nm}$ ranges. As shown in Figures 53 and 43, the concentration pattern of E02138 was similar to those of E02136 and E02140. The pattern was unique to the low cetane fuels, and it consisted of a nuclei mode that spanned from $5 \mathrm{~nm}$ to $70 \mathrm{~nm}$ and an accumulation mode that peaked between $70 \mathrm{~nm}$ and $300 \mathrm{~nm}$ and rose again in the $300 \mathrm{~nm}$ to $800 \mathrm{~nm}$ range. The test sequences administered with low cetane fuels provided an average run-to-run COV score of 90. Statistically, the use of low cetane-based fuels resulted in the most precise run-to-run particle concentration measurements.

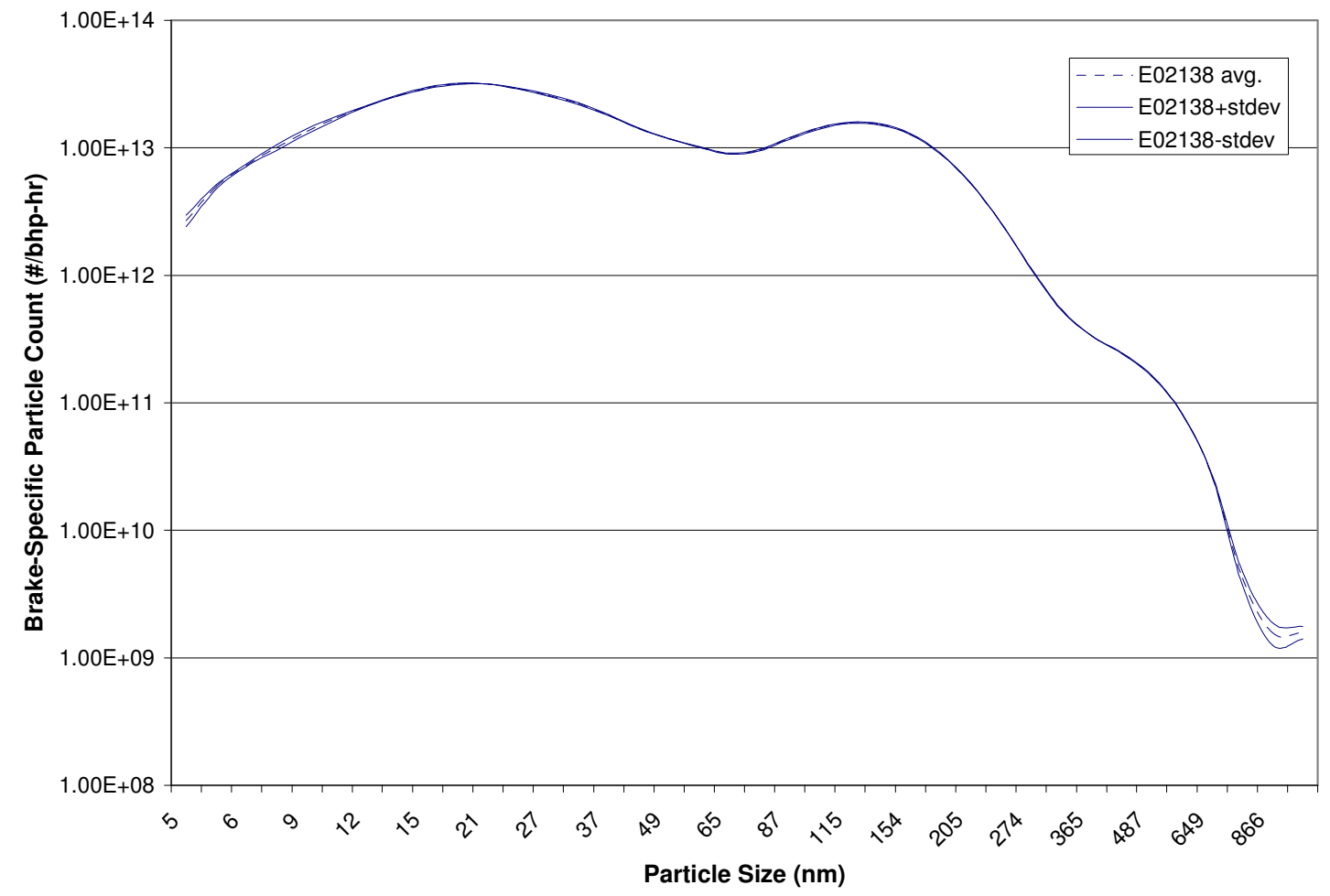

Figure 53 Average Particle Concentrations of Test Sequence E02138 
According to the $\mathrm{COV}$ scoring technique, the test sequence with the least repeatable results was E01921. The average particle concentrations and standard deviations of E01921 can be seen below in Figure 54. In the three tests of E01921, only the range of diameters between $21 \mathrm{~nm}$ and $205 \mathrm{~nm}$ had COVs of less than $10 \%$. With an average COV score of 185, the ISM 370 provided the least repeatable run-to-run results. Figure 54 illustrates that the E01921 particle concentration trend was of the typical "flat line" shape for the ISM 370. However, the standard deviations of E01921 were abnormally high, resulting in a COV score of 321.

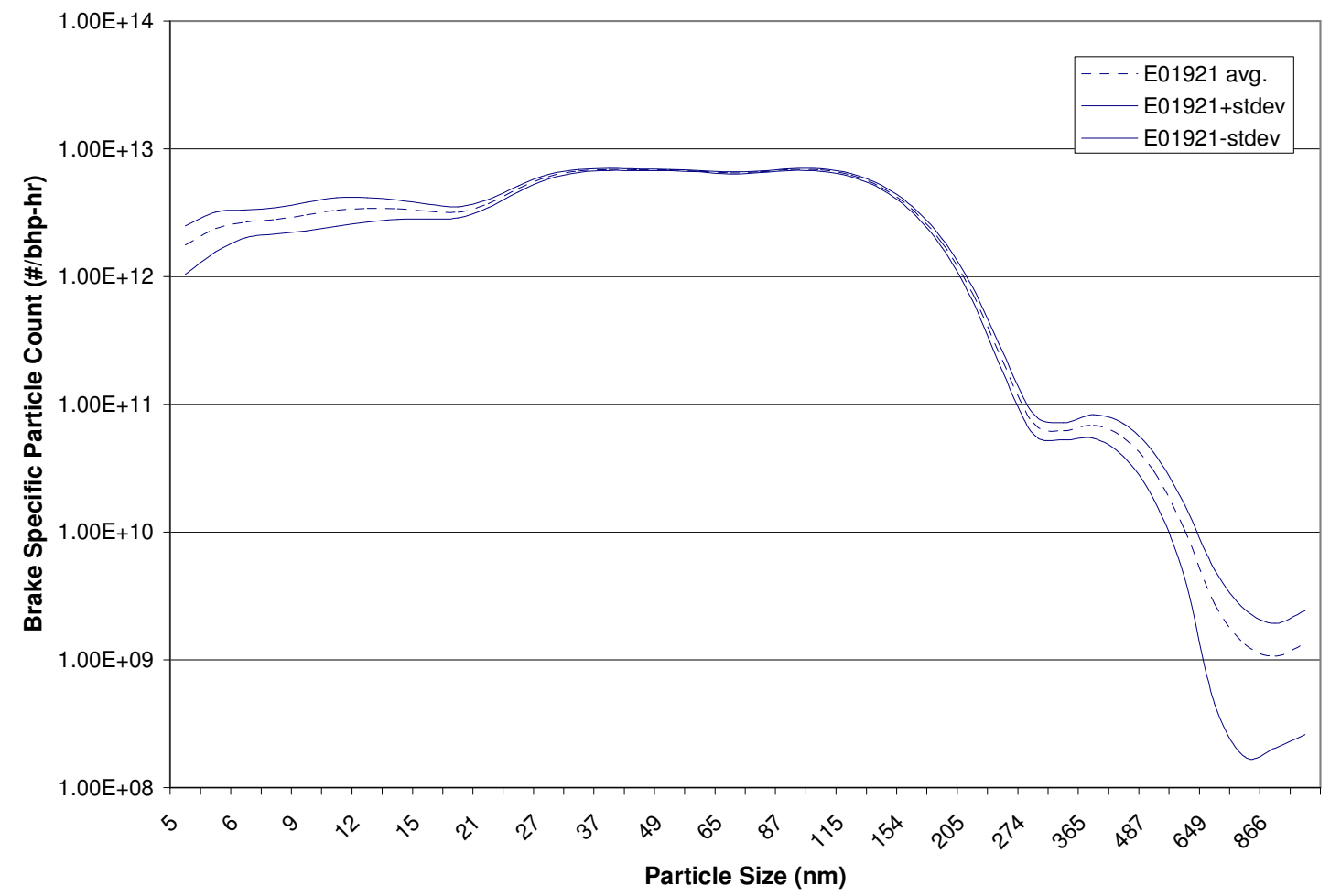

Figure 54 Average Particle Concentrations of Test Sequence E01921

For the particle concentration measurements taken with the DMS-500, test sequences E01928, E01921, E01977, E02099, E02138, and E01959 resulted in the highest and lowest run-to-run $\mathrm{COV}$ scores for the three engines used. In order to compare run-to-run repeatability between the DMS measurements and the rest of the 
laboratory data, $\mathrm{COV}$ values were calculated for the gaseous and PM mass emissions of the same six test sequences. These COV values, as well as the corresponding DMS scores, are listed below in Table 12.

Table 12 COVs of Laboratory Measurements and DMS COV Scores

\begin{tabular}{|c|c|c|c|c|c|c|c|c|c|c|c|}
\hline \multirow{2}{*}{$\begin{array}{c}\text { Test } \\
\text { Sequence }\end{array}$} & \multicolumn{10}{|c|}{ COV (\%) } & \multirow{2}{*}{$\begin{array}{c}\text { DMS coV } \\
\text { Score }\end{array}$} \\
\cline { 2 - 14 } & $\mathrm{HC}$ & $\mathrm{HC2}$ & LoCO & $\mathrm{HiCO}$ & $\mathrm{CO}_{2}$ & $\mathrm{NO}_{x}$ & $\mathrm{NO}_{\times} 2$ & $\mathrm{NO}_{2}$ & $\mathrm{TPM}$ & TEOM & \\
\hline E01928 & 0.44 & 1.03 & 0.90 & 0.80 & 0.17 & 0.17 & 0.20 & 0.39 & - & 0.63 & 107 \\
\hline E01921 & 0.08 & 0.63 & 2.08 & 1.34 & 0.20 & 0.57 & 0.02 & 0.00 & 2.53 & 2.08 & 321 \\
\hline E01977 & 0.95 & 0.97 & 0.06 & 0.52 & 0.09 & 0.13 & 0.01 & 0.07 & 0.62 & 0.49 & 117 \\
\hline E02099 & 0.87 & 2.02 & 0.24 & 0.31 & 0.20 & 0.32 & 0.36 & - & 1.85 & 1.61 & 246 \\
\hline E02138 & 0.21 & 0.04 & 0.47 & 0.82 & 0.05 & 0.12 & 0.03 & - & 0.57 & 0.41 & 41 \\
\hline E01959 & 2.36 & 3.59 & 0.08 & 0.54 & 0.15 & 0.31 & 0.23 & 0.16 & 0.47 & - & 156 \\
\hline
\end{tabular}

As in the particle concentration results described above, E01928 and E01921 corresponded to the most and least repeatable tests performed on the ISM 370, E01977 and E02099 were from the most and least repeatable tests on the DDC S60, and E02138 and E01959 included the most and least repeatable tests completed with the rebuilt DDC S60. Only run-to-run variations from the $\mathrm{CO}_{2}$ and $\mathrm{NO}_{\mathrm{x}}$ measurements showed any relation to the COV scores in Table 12. For example, between E01928 and E01921, $\mathrm{CO}_{2}$ showed a COV increase from $0.17 \%$ to $0.20 \%, \mathrm{NO}_{\mathrm{x}}$ showed a COV increase from 0.17 $\%$ to $0.57 \%$, and the DMS showed a COV score increase from 107 to 321 . In that $\mathrm{CO}_{2}$ and $\mathrm{NO}_{\mathrm{x}}$ COVs were higher where $\mathrm{COV}$ scores increased, $\mathrm{CO}_{2}$ and $\mathrm{NO}_{\mathrm{x}}$ results were similar to particle concentration results. Since $\mathrm{NO}_{\mathrm{x}}$ and $\mathrm{CO}_{2}$ measurements tend to vary as particle concentration data varies, it is evident that $\mathrm{NO}_{\mathrm{x}}, \mathrm{CO}_{2}$, and $\mathrm{PM}$ emissions are related. All other laboratory measurements seemed to display random patterns of variation in relationship to the COV scores. 


\subsubsection{Comparison of TEOM and DMS Data}

Equations 2 through 8, which were used to correct for water absorption on the oscillating TEOM filter, are included and discussed in the Literature Review section of this thesis. Effective particle densities, given by $\rho_{\text {eff }}$, for the DMS data were calculated with Equation 13, which is based on the approximation that particles of each diameter are spherical.

$$
\rho_{\text {eff }}=1.2+b_{1} * \sin \left(\left(\frac{d}{800}\right)+b_{2} * \pi\right) \quad \text { Equation } 13
$$

The linear effective density curve of approximately $1.1 \mathrm{~g} / \mathrm{cc}$ at $5 \mathrm{~nm}$ and $0.20 \mathrm{~g} / \mathrm{cc}$ at $1000 \mathrm{~nm}$, as described in Lappi's research in the Literature Review section, was not used in this study. Instead, the effective particle density equation above was created. Eq. 13 was based on the downward sweep of a sine wave, and it generates a density curve that also ranges from about $1.1 \mathrm{~g} / \mathrm{cc}$ to $0.20 \mathrm{~g} / \mathrm{cc}$. The symbol $d$ stands for the equivalent momentum diameter of the particle, and $b_{1}$ and $b_{2}$ are constants. The constants used in Equations 2 and 13 are provided below in Table 13.

Table 13 Water Absorption and Effective Density Coefficients

\begin{tabular}{|c|c|c|c|}
\hline$C_{1}$ & $C_{2}$ & $b_{1}$ & $b_{2}$ \\
\hline 0.072 & 0.012 & 1.04 & 1.03 \\
\hline
\end{tabular}

The values of $C_{1}$ and $C_{2}$ originated from Jarrett's work (which is summarized in the Literature Review) in the Evaluation and correction of moisture adsorption and desorption from a tapered element oscillating microbalance. Adjustable coefficients $b_{1}$ and $b_{2}$ were used to "fine tune" the effective densities until a best-fit curve was determined for the following comparisons. 
The effective density curve of Equation 13 is apparent below in Figure 55. These effective particle densities were used to convert particulate matter concentration data of the DMS into continuous PM mass data. Although particles that are greater than $50 \mathrm{~nm}$ are approximated to be more than $99 \%$ carbon, their densities continuously decrease as diameter increases [24]. This is because their equivalent diameters result in increasingly greater volume differences between the "actual particle" and the "equivalent particle." A concave shape was chosen for the effective density curve because of the assumption that particle density does not go to zero as particle size increases past $1000 \mathrm{~nm}$.

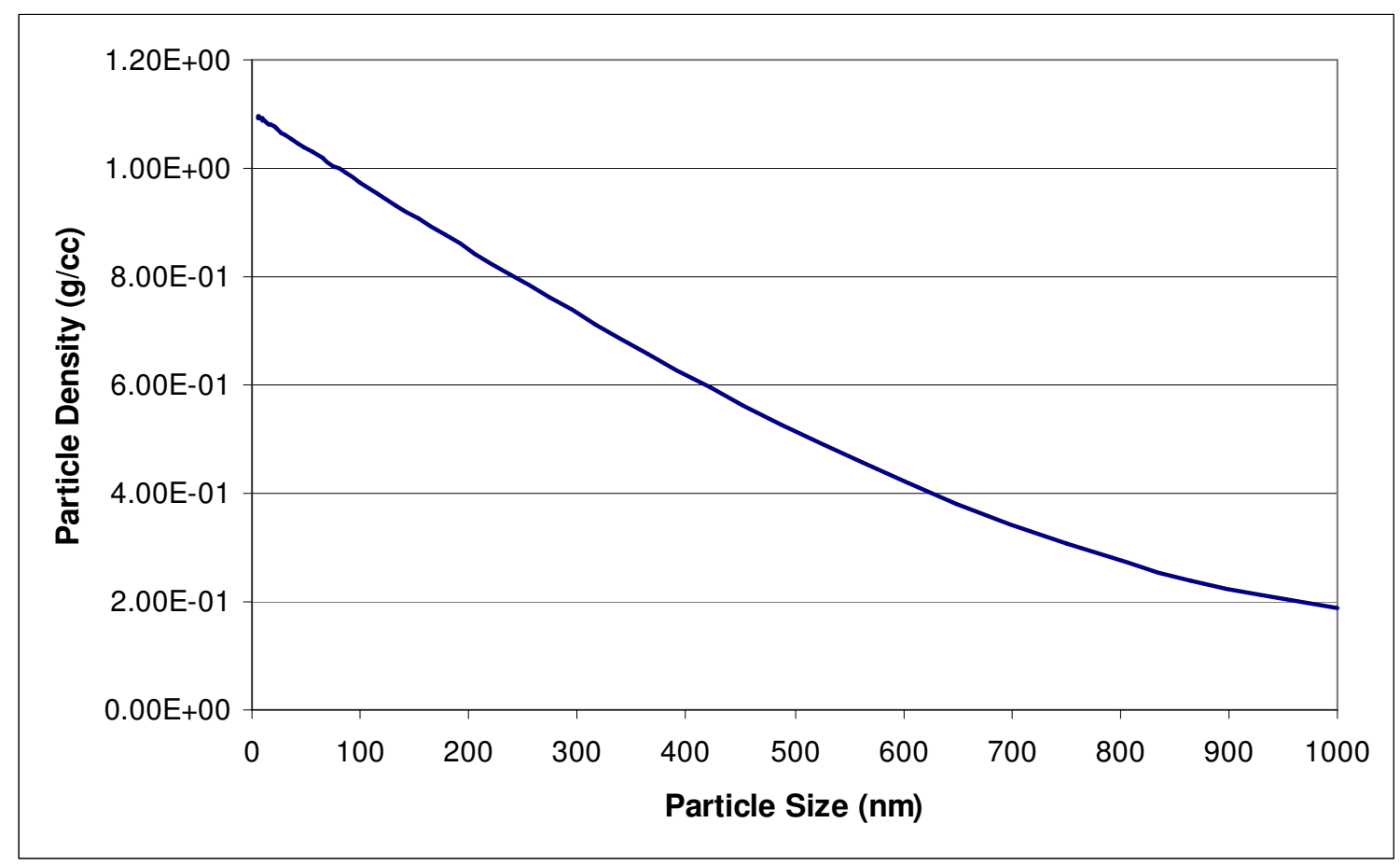

Figure 55 Effective Particle Densities

Mass values were calculated from effective particle densities using the spherical particle volumes shown in Figure 56. The particle volumes in Figure 56 increase cubically as particle diameters increase. The reason for approximating particles to be 
spherical in shape is discussed in the Experimental Setup and Procedures section on page 55.

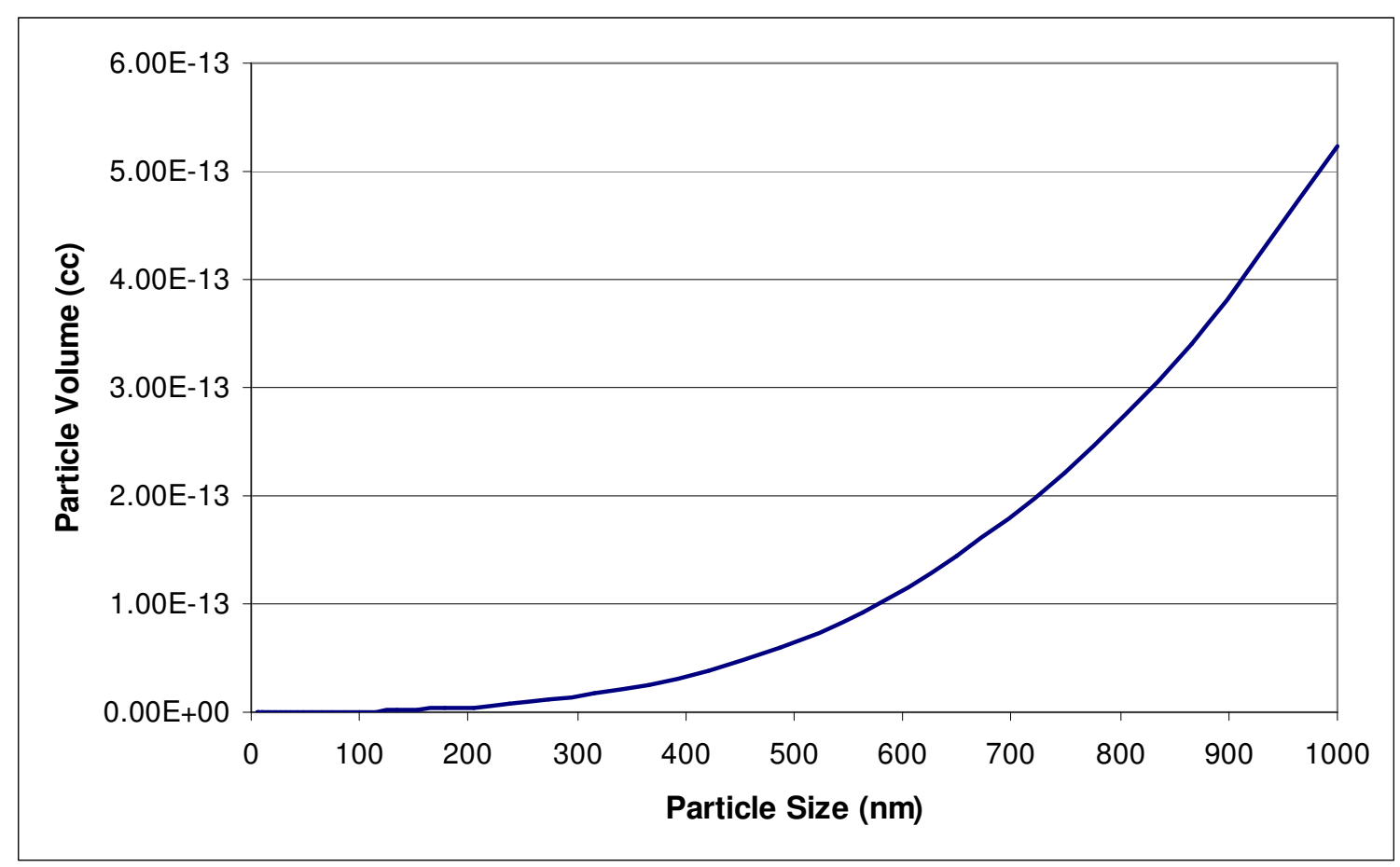

Figure 56 Particle Volumes Based on Spherical Shape

Particle mass, which is displayed in Figure 57, was calculated by multiplying effective density by volume. The approximated particle masses range from $8 * 10^{-20} \mathrm{~g}$ for the particles that are $5 \mathrm{~nm}$ in diameter to $1 * 10^{-13} \mathrm{~g}$ for the $1000 \mathrm{~nm}$ particles. This is a theoretical fit for the conversion of particle concentration to particle mass. An actual particle mass curve might be dissimilar in shape, especially in the nuclei mode range where volatile substances exist within the PM. 


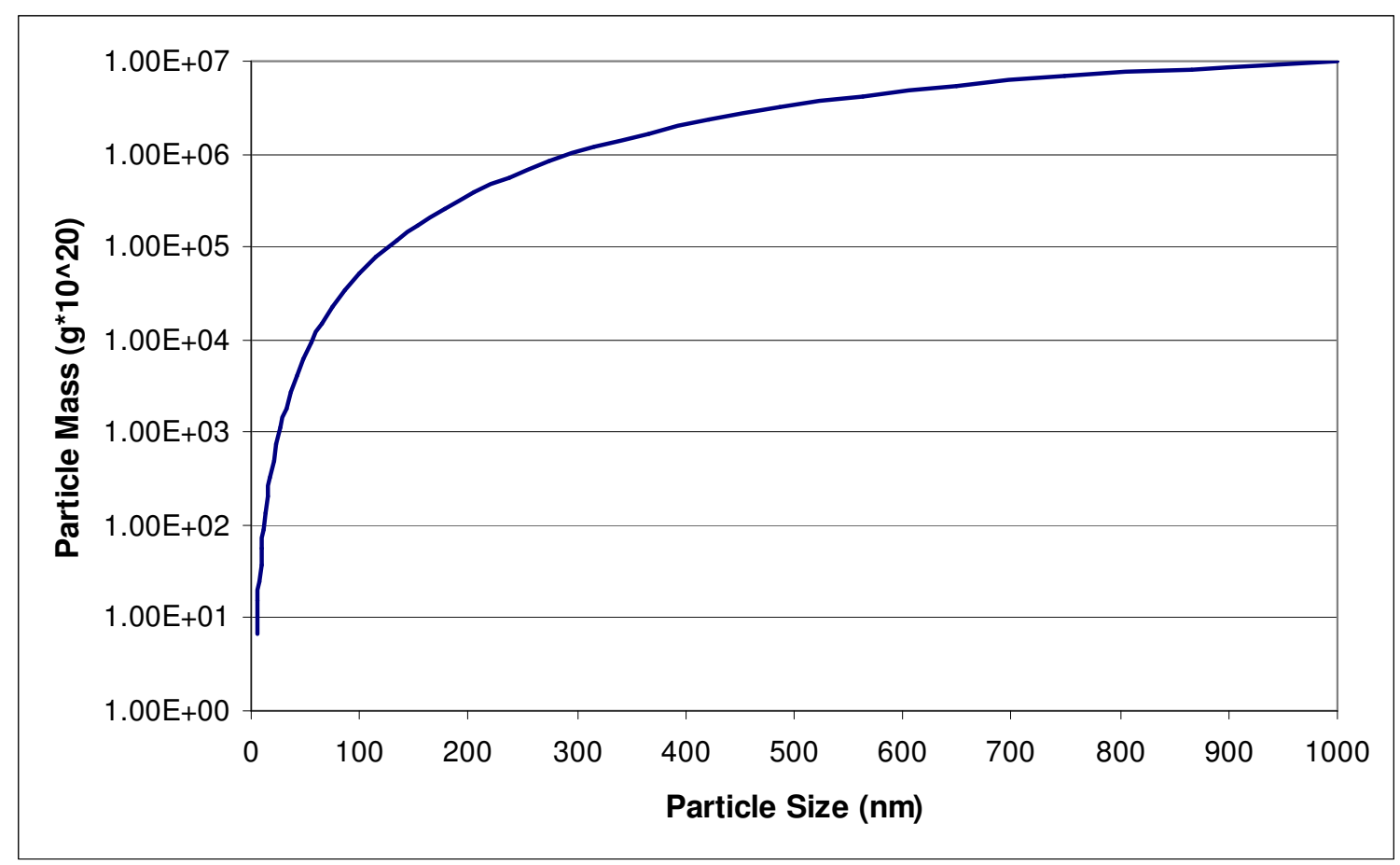

Figure 57 Approximate Particle Masses

Test E02123_02 was performed on the rebuilt DDC S60 with PONCA diesel fuel. The uncorrected continuous particle distribution of E02123_02, as measured with the DMS, is illustrated on page 107 in Figure 58. Figure 59, also on page 107, shows the continuous mass rate of PM through the TEOM. It was placed directly below Figure 58 so that a visual association between particle number and particle mass emissions could be made. Note that the spectral density is the same as the diluted volumetric particle concentration multiplied by $16\left((\mathrm{dN} / \mathrm{dlogdp}) / \mathrm{cc}=16^{*}\right.$ particles/cc $)$. The PM mass rate, as illustrated by Figure 58, during nanoparticle production is approximately zero. During periods of increased engine loading, such as between 800 seconds and 860 seconds, 100 nm particle production reaches up to $1 * 10^{7}(\mathrm{dN} / \mathrm{dlogdp}) / \mathrm{cc}$ and mass rate measured by the TEOM can be as high as $37 \mathrm{mg} / \mathrm{s}$. 
The effect of engine speed and load on particle number emissions is discussed in the Continuous FTP Data section on pages 120 through 122. A correlation between particle number emissions and PM mass emissions was made for E02123_02, and it is discussed throughout the rest of this section. The production of PM was never negative, but moisture evaporation from the oscillating TEOM filter caused readings of negative mass deposition. Therefore, an attempt to find the actual PM mass rate through the TEOM was made. The line titled TEOMorig in Figure 59 represents the original TEOM mass data of E02123_02. TEOMcor1 corresponds to the TEOM mass rate after corrections were carried out for the water absorption/desorption on the TEOM filter. The positive portions of the corrected mass-rate line should generally be lower than the positive parts of the uncorrected line, because water was being deposited on the filter faster than it could evaporate. Similarly, the negative parts of the corrected line should be higher than those of the uncorrected line because water was evaporating more quickly than it was being deposited. The corrected line should be consistently lower in magnitude than the uncorrected line, but theoretically, it must provide the same average mass rate. In this case, the corrected mass rate line was more accurate than the uncorrected line, but it was not ideal. This is apparent because there were still negative values on portions of the corrected line, but they were usually less negative than values on the same portions of the uncorrected line. In short, the water correction technique that was used was not entirely effective. 


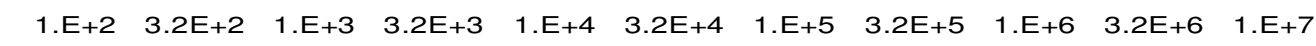

Spectral Density ((dN/dlogdp)/cc)

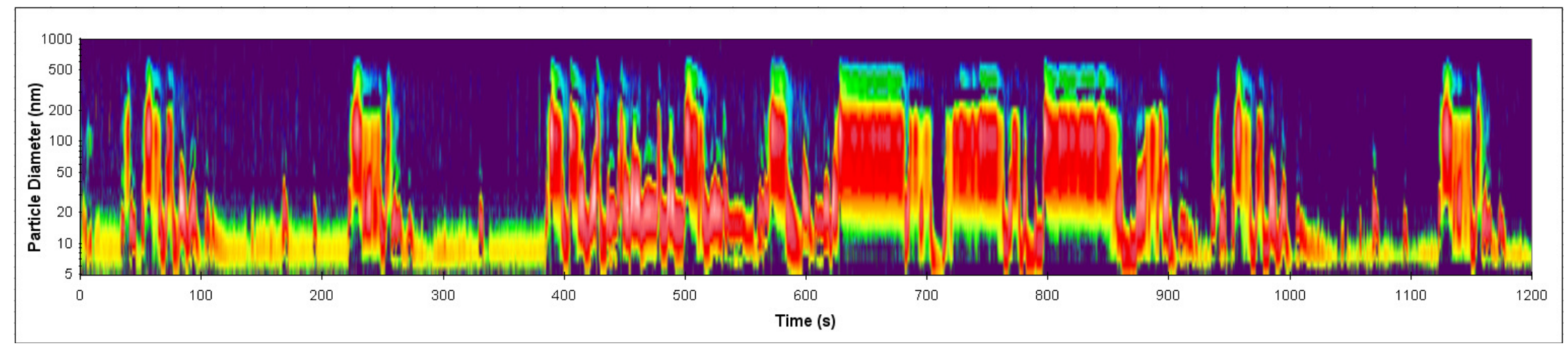

Figure 58 Continuous Particle Distribution of E02123_02 as Measured with the DMS-500

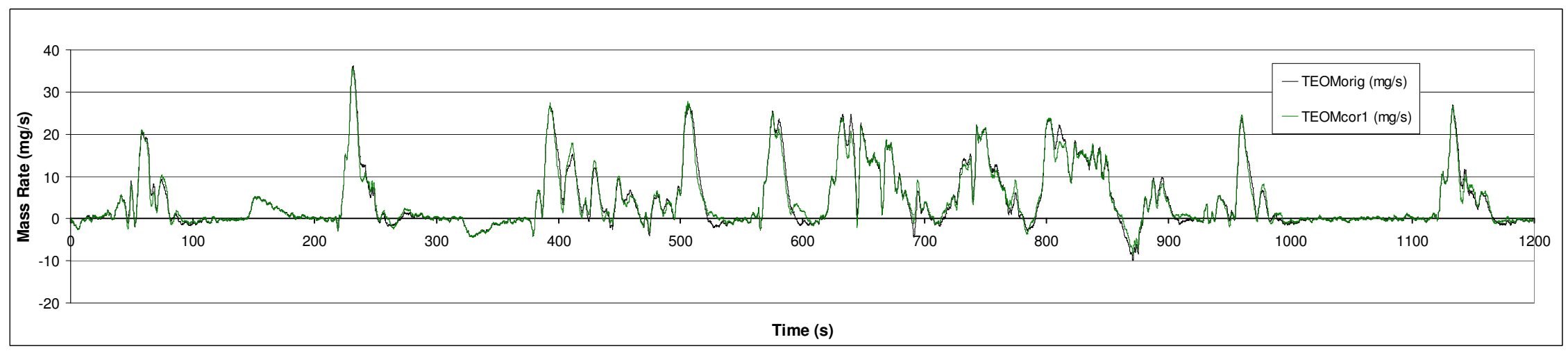

Figure 59 PM Mass-Rate of E02123_02 as Measured with the TEOM 
In Jarrett and Clark's Evaluation of Methods for Determining continuous Particulate Matter from Transient Testing of Heavy-Duty Diesel Engines, it was documented that a TEOM typically accounted for $82 \%$ of the mass measured by particulate filters. In the case of E02123_02, the total PM mass calculated from the TEOM data was $5.189 \mathrm{~g}$. The PM collected on a gravimetric filter indicated that the total amount of PM emitted from E02123_02 weighed 5.767 g. Over this test, the TEOM accounted for $90 \%$ of the PM mass. The corrected and uncorrected mass-rate data from the TEOM resulted in the "TEOMorig accum." and the "TEOM cor1 accum." lines of Figure 60. The entire curve of TEOMcor1 was divided by 0.90 ; and it was used to calculate the other accumulation curve, "TEOMcor2 accum.," of Figure 60. This was done so that the PM accumulation of the TEOM shown by TEOM cor2 accum. would match the total particulate matter (TPM) of the gravimetric filter. At any given point, TEOMorig accum. and TEOMcor1 accum. are less than $1 \%$ different, and TEOMcor2 accum. is approximately $10 \%$ greater than TEOMorig accum. and TEOMcor1 accum. The total amount of PM accumulated according to TEOMcor2 accum. was $5.775 \mathrm{~g}$, which was $0.14 \%$ greater than the TPM of the gravimetric filter. 


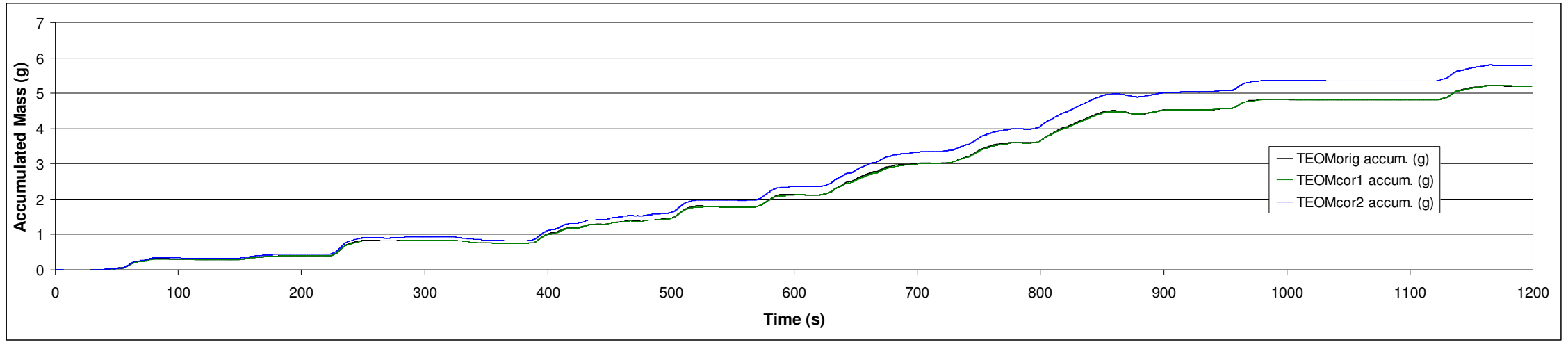

Figure 60 Corrected and Uncorrected PM Mass Accumulation of E02123_02 as Measured with the TEOM 
The curve, TEOMcor2, in Figure 61 corresponds to TEOMcor2 accum. in Figure 60; and it is essentially TEOMcor 1 from Figure 59 divided by 0.90 . This final version of PM mass-rates through the TEOM was compared to the calculated PM mass-rates through the DMS. The "DMS" mass-rates in Figure 61 were calculated using Equations 14 and 15.

$$
\begin{gathered}
\dot{m}=Q_{\text {total }} * C^{*} m \\
\dot{M}=\sum \dot{m}
\end{gathered}
$$

\section{Equation 14}

\section{Equation 15}

In Equation 14, $\dot{m}$ stands for the mass flow-rate, in grams per second, of particles of a single diameter. The symbol $Q_{\text {total }}$ represents the flow-rate within the primary dilution tunnel in standard cubic centimeters per second. The concentration of a given sized particle is characterized by $C$, where $C$ is the measured concentration (particles/cc) multiplied by 1.5 to correct for the secondary dilution ratio and the DMS data file factor. The lower case $m$ stands for the theoretical mass, in grams, of each particle of a specific diameter. Equation 15 is used to calculate the total mass-flow of PM at any given time, which is represented by $\dot{M}$ and given in grams per second. The term $\sum \dot{m}$ corresponds to the summation of instantaneous mass flow-rates (in grams per second) for the particles of each diameter.

The DMS mass-rate line roughly follows the TEOM line, but it appears to spike irregularly. These spikes were caused by impulses in PM emissions, which were due to turbo lag. For example, the $90 \mathrm{mg} / \mathrm{s}$ spike at 230 seconds occurred when the engine suddenly switched from $0 \mathrm{ft}-\mathrm{lb}$ at idle to approximately $1200 \mathrm{ft}-\mathrm{lb}$ at wide open throttle 
(WOT). Sudden boosts in PM are also visible in Figure 58 during the first few seconds of each span of time where an accumulation mode appears. Possibly because of a moving average within the data analysis or turbulence in the secondary dilution tunnel, the TEOM failed to detect spikes in PM due to turbo lag. Apart from the spikes, the DMS line in Figure 61 differs from the TEOM line in that it is generally less positive and it is never negative. It seems that use of the DMS and the preceding technique for converting PM concentration to PM mass is a way to measure PM mass-rate without the issue of moisture interference. 


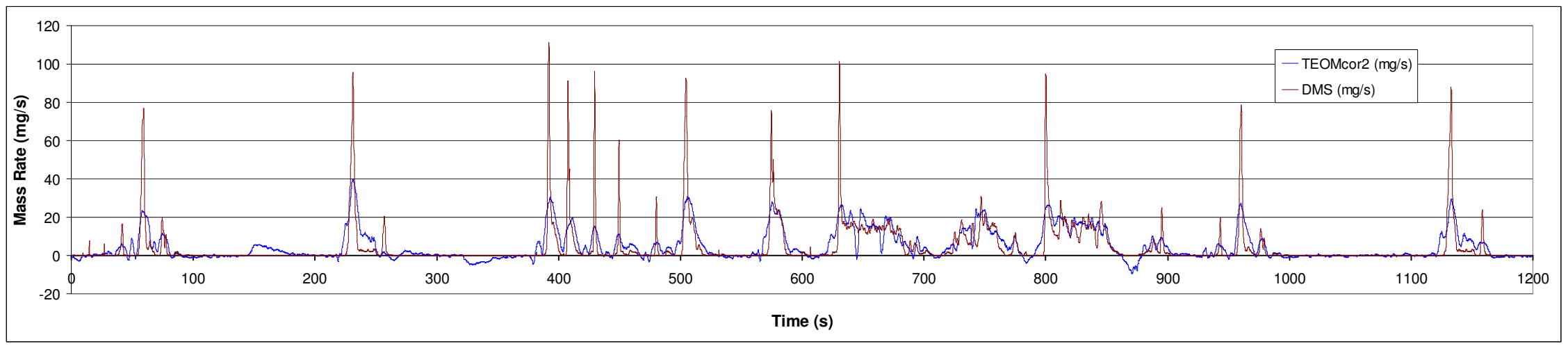

Figure 61 PM Mass-Rates of E02123_02 as Measured with the TEOM and the DMS 
A curve for the continuous accumulation of PM mass during E02123_02 was derived from the DMS mass-rate line of Figure 61. It is labeled "DMS accum.," and it is shown in Figure 62. The double corrected TEOM accumulation line of Figure 60 was plotted against the DMS accumulation line in Figure 62. According to the adjusted TEOM curve, the effective density technique used for converting original DMS data into PM mass accumulation data was accurate to $4 \%$ or less at all points during the test E02123_02. 


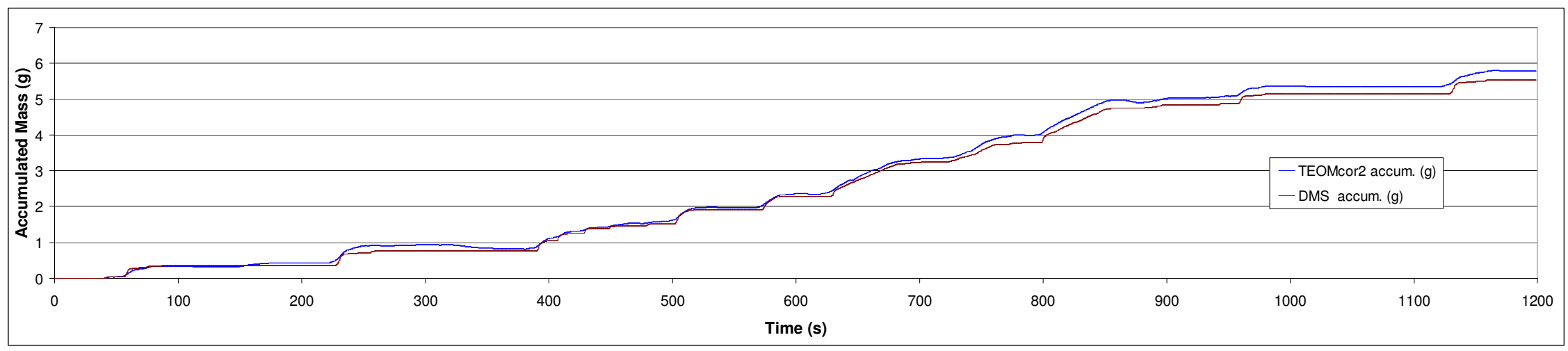

Figure 62 PM Mass Accumulation of E02123_02 as Measured with the TEOM and the DMS 
Over the hot-start FTP E02123_02, there was a $-3.85 \%$ difference between the calculated total PM mass of the DMS and the total particulate matter (TPM) of the gravimetric particulate filter. The converted DMS data of E02123_02 indicated a total PM amount of $5.545 \mathrm{~g}$. PM mass results between the TEOM, DMS, and gravimetric filters were found for eight other hot-start FTP tests using the same techniques as for E02123_02. A summary of the results is presented in Table 14. 
Table 14 Total PM Mass Results of TEOM, DMS (Converted from Volumetric Concentrations), and Gravimetric Filters

\begin{tabular}{|c|c|c|c|c|c|c|c|c|c|}
\hline Test Engine & Test No. & $\begin{array}{c}\text { Total Work } \\
\text { (bhp-hr) }\end{array}$ & $\begin{array}{l}\text { Average Tunnel } \\
\text { Flow (scfm) }\end{array}$ & $\begin{array}{l}\text { TEOM } \\
\text { (g) }\end{array}$ & $\begin{array}{l}\text { TEOMcor.1 } \\
\text { (g) }\end{array}$ & $\begin{array}{c}\text { TEOMcor.2 } \\
\text { (g) }\end{array}$ & TPM (g) & DMS (g) & $\begin{array}{l}\text { DMS vs TPM } \\
\text { Difference (\%) }\end{array}$ \\
\hline \multirow{3}{*}{ ISM 370} & E01920_02 & 26.43 & 2186 & 1.593 & 1.600 & 1.968 & 1.960 & 1.443 & -26.4 \\
\hline & E01927_03 & 26.44 & 2184 & 1.579 & 1.586 & 2.205 & 2.197 & 1.798 & -18.2 \\
\hline & E01929_04 & 26.41 & 2184 & 1.672 & 1.679 & 2.418 & 2.411 & 1.838 & -23.8 \\
\hline \multirow{3}{*}{ DDC S60 } & E01967_04 & 23.65 & 2210 & 5.000 & 5.008 & 6.191 & 6.182 & 6.284 & 1.66 \\
\hline & E01968_04 & 23.62 & 2204 & 5.073 & 5.081 & 6.320 & 6.312 & 6.563 & 3.98 \\
\hline & E01976_04 & 24.20 & 2190 & 4.504 & 4.513 & 5.423 & 5.414 & 5.531 & 2.16 \\
\hline \multirow{3}{*}{$\begin{array}{l}\text { Rebuilt DDC } \\
\quad \text { S60 }\end{array}$} & E02123_02 & 25.00 & 2187 & 5.189 & 5.197 & 5.775 & 5.767 & 5.545 & -3.85 \\
\hline & E02124_02 & 25.01 & 2184 & 4.960 & 4.968 & 5.449 & 5.441 & 5.675 & 4.30 \\
\hline & E02125_02 & 25.00 & 2180 & 5.112 & 5.120 & 5.540 & 5.532 & 5.877 & 6.24 \\
\hline
\end{tabular}


The eight tests summarized in Table 14 were chosen so that the DMS mass conversion technique could be tested on several different fuel types and engine technologies. Using the ISM 370, E01920_02 and E01929_04 were performed with NBB petroleum diesel fuel, and E01927_03 was performed with a $10 \%$ biodiesel/90\% petroleum diesel fuel blend. All of the fuels used for the other six tests were petroleum based, and only the fuels used in E01976_04 and E02124_02 were additized. The fuel names can be found in Table A-1 of the Appendix. TPM indicated by gravimetric filters was used as a standard, and the DMS mass conversions resulted in the following errors in total PM mass: 1.66 \% in E01967_04, 3.98 \% in E01968_04, 2.16 \% in E01976_04, $3.85 \%$ in E02123_02, $4.30 \%$ in E02124_02, and 6.24 \% in E02125_02. These errors were all considered to be low, but errors in the DMS mass conversion results were between $-18.2 \%$ and $-26.3 \%$ for the ISM 370. The information in Table 14 implies that the DMS mass conversion technique is quite accurate for FTPs of any fuel type on a 1992 Detroit Diesel DDC S60. With the 2004 Cummins ISM 370, however, the technique consistently provided total mass values with large negative errors, regardless of fuel type. It is possible that the use of EGR on the ISM 370 causes particle geometries to change throughout the spectrum. In such an event, there would be a need for new effective particle densities. The effective particle density curve shown in Figure 55, if altered for the ISM 370, would result in much closer correlation between total PM mass data of the DMS and TPM data from gravimetric filters. 


\subsubsection{Continuous FTP Data}

The ISM 370 and NBB Cand. fuel were used during the test sequence E01920. Continuous data from each of the three hot-start FTPs in E01920 is presented below; and relationships of the distributions and concentrations of particles to engine speed, torque, overall dilution ratio, and residence time are discussed. The run-to-run COVs of E01920 are shown in Figure 63. Several contributing factors to variation are discussed within this section.

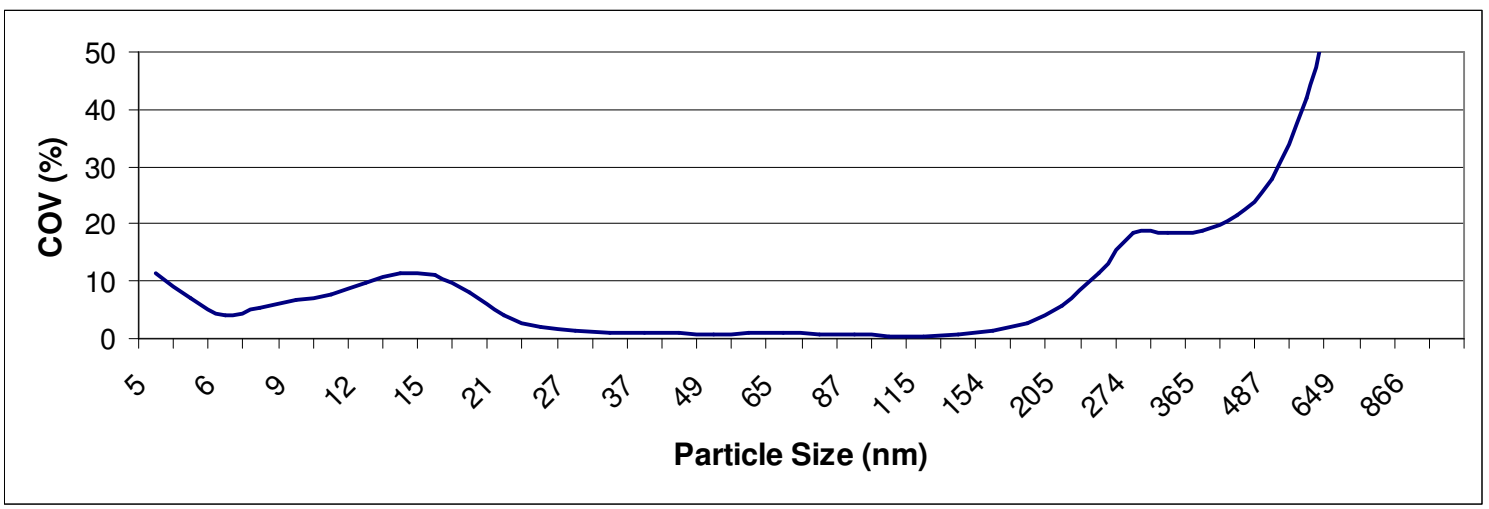

Figure 63 Run-to-Run Variation of the E01928 Test Sequence

There are many engine operating events that take place during an FTP that have unique particle distributions. Figure 64 illustrates the average engine speed and torque curves for test sequence E01920. In the discussion following Figure 64, six basic particle distribution occurrences are matched with their respective engine speeds and torques. The six circled speed and torque points in Figure 64 are: A (1500 rpm and $600 \mathrm{ft}-\mathrm{lb}$ @ 105 seconds), B (760 rpm and -60 ft-lb @ 215 seconds), C (1250 rpm and 1250 ft-lb @ 270 seconds), D (700 rpm and 0 ft-lb @ 350 seconds), E (1600 rpm and 1250 ft-lb @ 675 seconds), and F (1800 rpm and -130 ft-lb @ 910 seconds). 


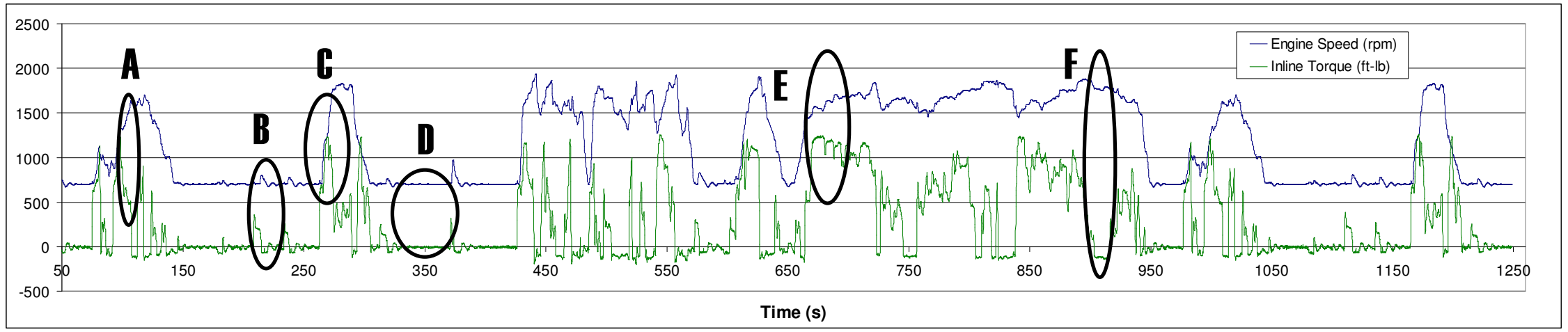

Figure 64 Averaged Engine Speed and Torque Curves of E01920 
The particle concentrations and distributions for E01920_02, E01920_03, and E01920_04 during these six speed and load points are circled on the following pages in Figures 65, 66, and 67 (respectively) and labeled correspondingly as A - F. The maximum speed and torque values during E01920 were $1850 \mathrm{rpm}$ and $1250 \mathrm{ft}-\mathrm{lb}$. The minimum speed of E01920 was $700 \mathrm{rpm}$, and the minimum torque was $-135 \mathrm{ft}-\mathrm{lb}$. A percent scale for speed is used in the following discussion, where $0 \%$ corresponds to minimum speed and $100 \%$ corresponds to maximum speed. A similar scale for load is also used, where $0 \%$ corresponds to zero load, $100 \%$ corresponds to maximum load, and - $10 \%$ corresponds to minimum load. The words "high" and "low," when used to describe engine speed or load, refer to $50 \%$ or more and $49 \%$ or less, respectively.

Point A illustrates that at $70 \%$ speed and $43 \%$ load, there was a distribution of particles that ranged from $15 \mathrm{~nm}$ to $200 \mathrm{~nm}$ and had a $1.2^{*} 10^{5}$ (dN/dlogdp)/cc peak at 50 nm. The results at point $A$ indicate that at a high engine speed and low load, there is typically a flat accumulation mode with a peak distribution of around $1.0^{*} 10^{5}$ $(\mathrm{dN} / \mathrm{d} \log \mathrm{dp}) / \mathrm{cc}$.

Shown by point B is that an operating condition of $5.2 \%$ speed and $-4.3 \%$ load led to a particle distribution that had a $1.2 * 10^{6}(\mathrm{dN} / \mathrm{dlogdp}) / \mathrm{cc}$ peak at $12 \mathrm{~nm}$ and ranged from $7 \mathrm{~nm}$ to $180 \mathrm{~nm}$. Point B signifies that under the condition of low engine speed and a small negative load, which occurs during light motoring of the engine, there is often a wide range of particles emitted; where a dominant nuclei mode is present and there is a large number of nanoparticles (over $1.0 * 10^{6}$ (dN/dlogdp)/cc) emitted at about $12 \mathrm{~nm}$.

At point C, $48 \%$ speed and $90 \%$ load resulted in a distribution of particles between $20 \mathrm{~nm}$ and $200 \mathrm{~nm}$ that peaked at $7.5^{*} 10^{6}(\mathrm{dN} / \mathrm{d} \operatorname{logdp}) / \mathrm{cc}$ around $120 \mathrm{~nm}$, and 
another range of particles between $300 \mathrm{~nm}$ and $600 \mathrm{~nm}$ that had a $3.3 * 10^{4}(\mathrm{dN} / \mathrm{dlogdp}) / \mathrm{cc}$ peak at $400 \mathrm{~nm}$. The information that point $\mathrm{C}$ provides is that engine operating conditions of low speed and high load generally result in an accumulation mode distribution with extremely high particle concentrations in the $100 \mathrm{~nm}$ to $130 \mathrm{~nm}$ size range and relatively high concentrations in the particles larger than $200 \mathrm{~nm}$. Such an event corresponds to the greatest mass-flow rate of PM through the exhaust pipe, and is typical when there is a sudden increase in load that leads to turbo lag and a low pitched "grumbling" noise of the engine.

Point D refers to $0 \%$ speed, $0 \%$ load, and a $5 \mathrm{~nm}$ to $18 \mathrm{~nm}$ particle distribution that had a $4.0 * 10^{4}(\mathrm{dN} / \mathrm{d} \log \mathrm{dp}) / \mathrm{cc}$ peak at $9 \mathrm{~nm}$. Point $\mathrm{D}$ is characterized by engine idle, with which there are normally low concentrations (less than $4.0^{*} 10^{4}$ (dN/dlogdp)/cc) of nanoparticles.

Shown by point E, operation at $78 \%$ speed and $90 \%$ load caused a distribution of particles between $10 \mathrm{~nm}$ and $400 \mathrm{~nm}$ that had a $3.0^{*} 10^{5}(\mathrm{dN} / \mathrm{d} \operatorname{logdp}) / \mathrm{cc}$ peak at $40 \mathrm{~nm}$. Point E depicts the event of a full-throttle hill climb or acceleration, where there is typically a wide accumulation mode that peaks at between $3.0^{*} 10^{5}(\mathrm{dN} / \mathrm{dlogdp}) / \mathrm{cc}$ and $4.0 * 10^{5}(\mathrm{dN} / \mathrm{d} \operatorname{logdp}) / \mathrm{cc}$.

There was a $96 \%$ speed and $-9.4 \%$ load condition at point $\mathrm{F}$, which yielded a particle distribution of $5 \mathrm{~nm}$ to $30 \mathrm{~nm}$ with a peak of $8.0^{*} 10^{6}(\mathrm{dN} / \mathrm{dlogdp}) / \mathrm{cc}$ at $8 \mathrm{~nm}$. The occurrences at and in the 15 seconds after point $\mathrm{F}$ are always present during heavy motoring of the engine when there is high engine speed and high negative torque. It was noted that after point $\mathrm{F}$, the engine speed and load remained constant for 15 seconds, but the particle distribution gradually changed from an $8.0 * 10^{6}(\mathrm{dN} / \mathrm{d} \log \mathrm{dp}) / \mathrm{cc}$ peak at $8 \mathrm{~nm}$ 
to a $7.0 * 10^{5}(\mathrm{dN} / \mathrm{d} \log \mathrm{dp}) / \mathrm{cc}$ peak at $5 \mathrm{~nm}$. The greatest concentrations of nanoparticles occur when there is a sudden shift from high power output to great energy absorption of the engine. Directly after such a shift in power, nanoparticle concentrations are high; but they decrease during constant motoring of the engine with exhaust temperature as the incylinder temperatures drop. At point $\mathrm{F}$, the exhaust temperature was recorded at $372{ }^{\circ} \mathrm{C}$. The exhaust temperature was recorded to be $240{ }^{\circ} \mathrm{C}$ at the end of the 15 second period of engine motoring, and the peak particle concentration was more than an order of magnitude lower than at point $\mathrm{F}$.

In Figures 65, 66, and 67, there are two areas of data where subtle differences were noticed. One area is labeled $\alpha$, and the differences within are illustrated by curved black lines. The other area has a large vertical rectangle around it, and it is labeled $\omega$. Specifically, these two areas are marked $\alpha 1$ and $\omega 1$ in E01920_02, $\alpha 2$ and $\omega 2$ in E01920_03, and $\alpha 3$ and $\omega 3$ in E01920_04. The differences within these areas are discussed below Figure 67. Data shown by the three figures below was aligned with engine speed. Since the three sets of data were not time-aligned, the timescales of each figure are different. The white lines labeled "AInput 1" are the raw voltage signals for engine speed. 


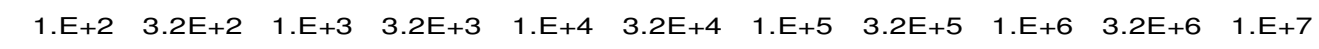
Spectral Density ((dN/dlogdp)/cc)

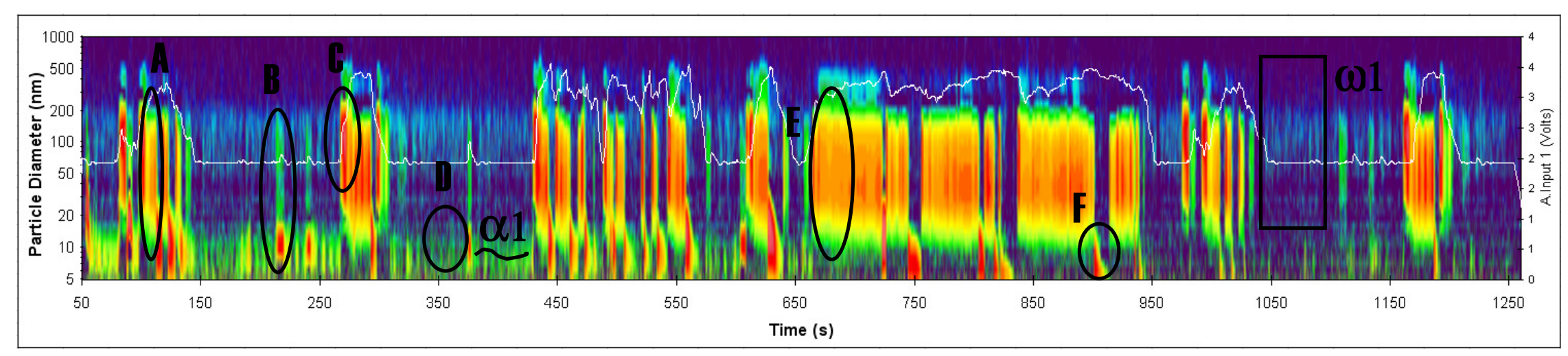

Figure 65 Continuous Particle Distribution of E01920_02 
One distinct difference in the particulate measurements of E01920_02, E01920_03, and E01920_04 can bee seen in Figure 66. A black block-arrow labeled $\pi$ points to a momentary distribution of particles ranging from $50 \mathrm{~nm}$ to $500 \mathrm{~nm}$ that have concentrations of between $1.2 * 10^{3}(\mathrm{dN} / \mathrm{d} \log \mathrm{dp}) / \mathrm{cc}$ to $5.0^{*} 10^{3}(\mathrm{dN} / \mathrm{dlogdp}) / \mathrm{cc}$. This brief emission of accumulation mode particles is illustrated by a vertical light blue stripe, and it is absent in the same section of E01920_02 and E01920_04. The sudden spike in particles could have been caused by one of many events; such as the detaching of soot packages from the walls of the exhaust pipe. 


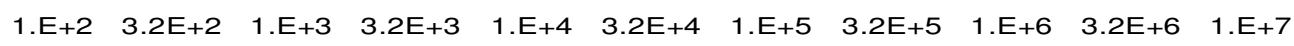
Spectral Density ((dN/dlogdp)/cc)

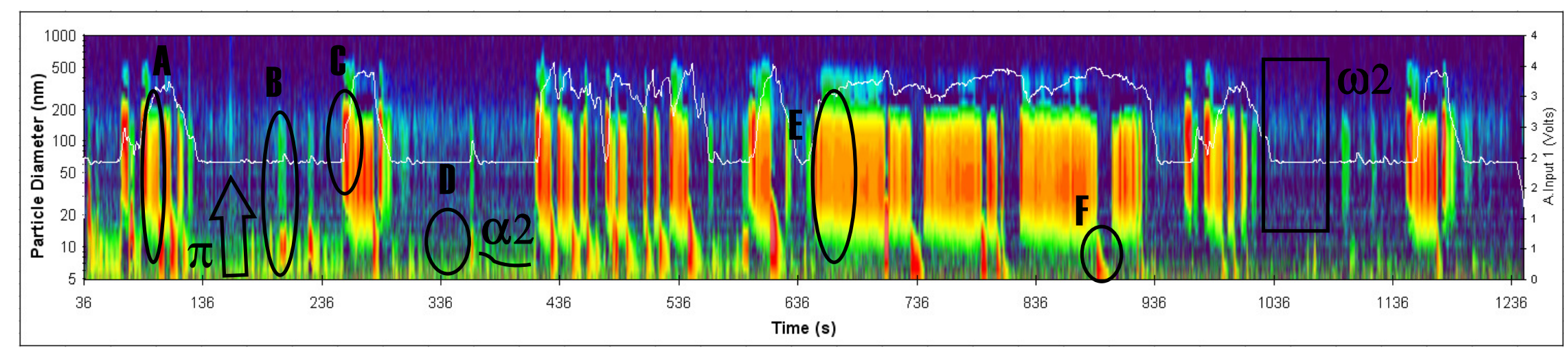

Figure 66 Continuous Particle Distribution of E01920_03 
Particles larger than $500 \mathrm{~nm}$ had concentrations that were usually less than, or slightly greater than, the lower detection limit of the DMS. This caused measurement errors that resulted in excessively high run-to-run variations for the particles above 500 nm. For E01920 and most other test sequences that were performed on the rebuilt DDC S60, there were run-to-run COV values of greater than $20 \%$ for the $500 \mathrm{~nm}$ to $1000 \mathrm{~nm}$ size range. Labeled $\sigma$, the horizontal rectangle in Figure 67 encloses a portion of data where accumulation mode particles were present but not great enough in concentration to be detected. The concentration at which "under ranging" began to take place was about $1 * 10^{3}(\mathrm{dN} / \mathrm{d} \operatorname{logdp}) / \mathrm{cc}$ 


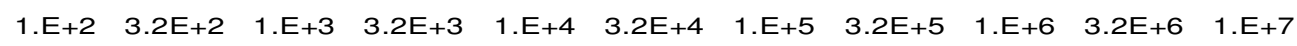
Spectral Density ((dN/dlogdp)/cc)

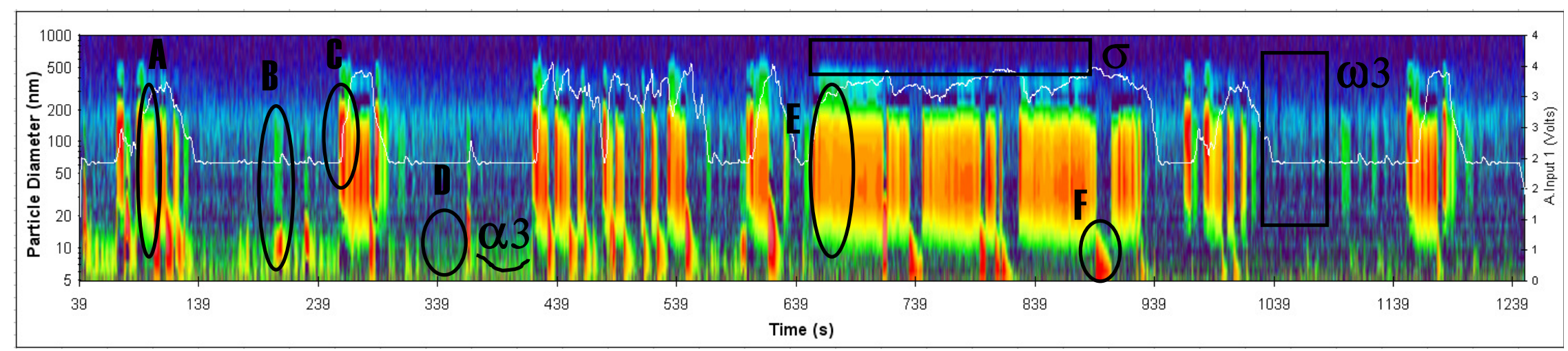

Figure 67 Continuous Particle Distribution of E01920_04 
Usually caused by control issues such as injection timing, engine "flutter" is an inconsistency in engine speed during idling conditions. Engine idle occurred during a 40 second time period about a third of the way through the FTPs. The curved black lines, $\alpha 1, \alpha 2$, and $\alpha 3$, of the three figures above represent the nuclei mode peaks during the same 40 seconds of each test. The $\alpha 1$ line of E01920_02 is shaped like an "s" curve. For E01920_03, the line labeled $\alpha 2$ is similar in shape to half of a parabola, and the $\alpha 3$ line of E01920_04 is shaped like a complete parabola. Each of these three peak indicating lines fell between the diameters of $7 \mathrm{~nm}$ and $10 \mathrm{~nm}$, but they were all unique in shape. The curves in the lines illustrate fluctuations in the modal distributions of nanoparticles during idle. It is likely that such fluctuations are caused by non-uniform fueling at idle, but there may be other contributing factors. As a result, run-to-run variation in nanoparticle concentration is affected by these fluctuations.

Within the boxes labeled $\omega 1, \omega 2$, and $\omega 3$, there is a light blue tint that indicates particle concentrations of between $1.0^{*} 10^{3}(\mathrm{dN} / \mathrm{d} \operatorname{logdp}) / \mathrm{cc}$ and $3.0^{*} 10^{3}(\mathrm{dN} / \mathrm{d} \log \mathrm{dp}) / \mathrm{cc}$. It is clear that the light blue color is least dominant in $\omega 1$ and most dominant in $\omega 3$. It was common for each consecutive test to appear to have greater concentrations within this area than the tests before. As in E01920_02, E01920_03, and E01920_04, the accumulation mode particles within the area labeled $\omega$ often appear to increase by almost an order of magnitude after only two or three tests. This is because of noise within the measurements due to soot accumulation on the electrometer rings of the DMS. Gradual soot buildup on the rings greatly affects accumulation mode particle concentration measurements during the test segment marked $\omega$. The run-to-run variations in accumulation mode particles, however, are only slightly affected. This is because over an 
FTP, the average accumulation mode concentrations (usually between $7.8^{*} 10^{4}$ $(\mathrm{dN} / \mathrm{d} \operatorname{logdp}) / \mathrm{cc}$ and $\left.1.3 * 10^{5}(\mathrm{dN} / \mathrm{d} \log \mathrm{dp}) / \mathrm{cc}\right)$ are changed by no more than $3.1 \%$ by an average increase of $2.5 * 10^{3}$ (dN/dlogdp)/cc (due to soot buildup).

Engine control during the first 300 seconds of an FTP is identical to control during the last 300 seconds. It is apparent in Figures 65, 66, and 67, however, that between the two identical test segments, particle concentrations throughout the nuclei mode are significantly dissimilar. In both segments, the particle size with the highest average concentration was $7.5 \mathrm{~nm}$. In the first 300 second segment, the average $7.5 \mathrm{~nm}$ particle concentration was $7.6^{*} 10^{4}(\mathrm{dN} / \mathrm{d} \operatorname{logdp}) / \mathrm{cc}$. During the last 300 second segment, the average concentration of $7.5 \mathrm{~nm}$ particles was $1.1 * 10^{4}(\mathrm{dN} / \mathrm{dlogdp}) / \mathrm{cc}$. The engine oil temperature during the first segment had an average value of $95.6{ }^{\circ} \mathrm{C}$. The average oil temperature during the other segment was $105{ }^{\circ} \mathrm{C}$. Since oil temperature was the only measured parameter between the two identical segments that was noticeably different, it can be deduced that oil temperature is largely responsible for the transformation of lube oil into nanoparticles. There were $86 \%$ fewer nanoparticles during the last 300 second segment, and since only oil could be linked to the difference, it is likely that at least $86 \%$ of all nanoparticle emissions from this engine and fuel combination can be attributed to engine oil.

All of the relationships between particle concentration measurements and engine speed, engine torque, exhaust temperature, engine flutter, under ranging, soot accumulation in the DMS, and engine oil temperature were the same for the other fuels that were tested on the rebuilt DDC S60. On both DDC S60 engines, only the 
magnitudes of these relationships changed from fuel to fuel. However, not all of the same correlations were observed when test sequences were executed with the ISM 370 .

Overall dilution ratio is related to DMS sampling issues such as under ranging and soot accumulation. During idle, mass-flow rate in the exhaust pipe was at a minimum, and the overall dilution ratio was normally about 445 . This was the highest dilution ratio during each FTP, and it often resulted in some under ranging in the nuclei mode particle range. The lowest dilution ratio during FTPs, which was usually around 63.6, occurred during full throttle when the mass-flow rate of raw exhaust was greatest. Since the mass-flow rate of the primary dilution air was nearly constant, changes in exhaust flow resulted in variations of the overall dilution ratio. For example, an increase in raw exhaust flow leads to a decrease in the overall dilution ratio. The overall dilution ratio, which had an average value of 175 , was calculated using the following equation.

$$
R_{o}=\left(\frac{Q_{\text {tunnel }}}{Q_{\text {raw }}}\right) * R_{s} \quad \text { Equation } 16
$$

From equation $16 ; R_{o}$ is the overall dilution ratio, $Q_{\text {tunnel }}$ is the standardized flow rate in the primary dilution tunnel $(\mathrm{scfm}), Q_{\text {raw }}$ raw is the standardized flow rate of raw exhaust (scfm), and $R_{s}$ is the secondary dilution ratio. Calculation of the secondary dilution ratio is discussed in the Secondary Dilution Ratio Validation section of this thesis. Average variations in the overall dilution ratios of test sequence E01920 are shown in Figure 68. Overall dilution ratio is directly related to particle mass rate at the sample intake of the DMS. If the overall dilution ratio were somehow forced to be constant at 445; PM mass rate into the DMS would be the same during idle, but it would be anywhere from $0 \%$ to $85 \%$ lower the rest of the time. 


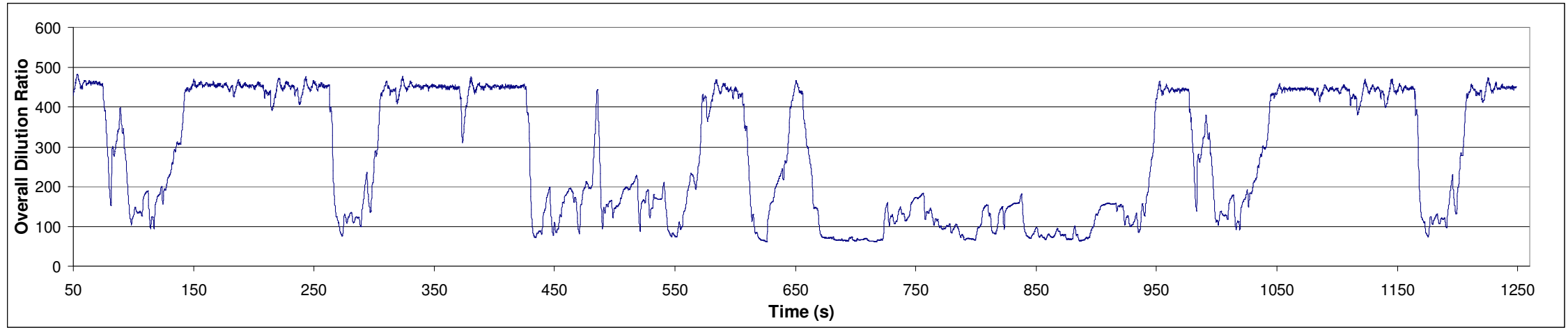

Figure 68 Averaged Overall Dilution Ratio Curve of E01920 
For the same reasons that dilution ratio varied, residence time varied. Residence time is a known factor in nanoparticle formation. The effects of residence time on particle distribution were not studied for this thesis, because with the chosen sampling system, it was not possible to control residence time. In fact, during FTPs, residence time for DMS measurements typically varied from 2.66 seconds to 4.38 seconds. Residence time was calculated by adding the amounts of time required for aerosol to go through the exhaust pipe, dilution tunnel, secondary dilution system, and DMS sampling line. The residence time from the exhaust pipe to the dilution tunnel ranged from 0.11 seconds during full throttle to 1.43 seconds during idle. In the dilution tunnel, volumetric flow rate varied slightly because of internal temperature variation, and residence time was consistently between 1.21 seconds and 1.62 seconds. The combined residence time of the heated sample line and secondary dilution system was constant at 0.26 seconds, and the residence of the DMS sampling line was 1.07 seconds. Each of these residence times were calculated using equation 17 , below.

$$
\Delta t=Q * \pi * r^{2} * l \quad \text { Equation } 17
$$

The symbol $\Delta t$ represents residence time, $Q$ denotes volumetric flow rate, and $r$ and $l$ stand for radius and length of the pipe, tunnel, or tube. The longest overall residence time occurred during idle, and the shortest occurred during full throttle. 


\subsection{SET RESULTS}

Supplemental emissions tests were used in this study to determine particle concentrations during various steady-state engine operating conditions. Each steady-state session during a SET can be identified by its unique operating condition. Mode 1 is the first segment, in which engine torque is zero. Modes 2 through 13 have equivalent timespans, and they transpire in order. The engine speed and torque curves of E01948_01 are shown as an example in Figure 69. It is apparent in Figure 69 that some of the modes share a common engine speed, but they all have unique torques. Although it appears that there is an instantaneous step change from one mode to another, there is actually a ramp transition that is not shown by Figure 69. 


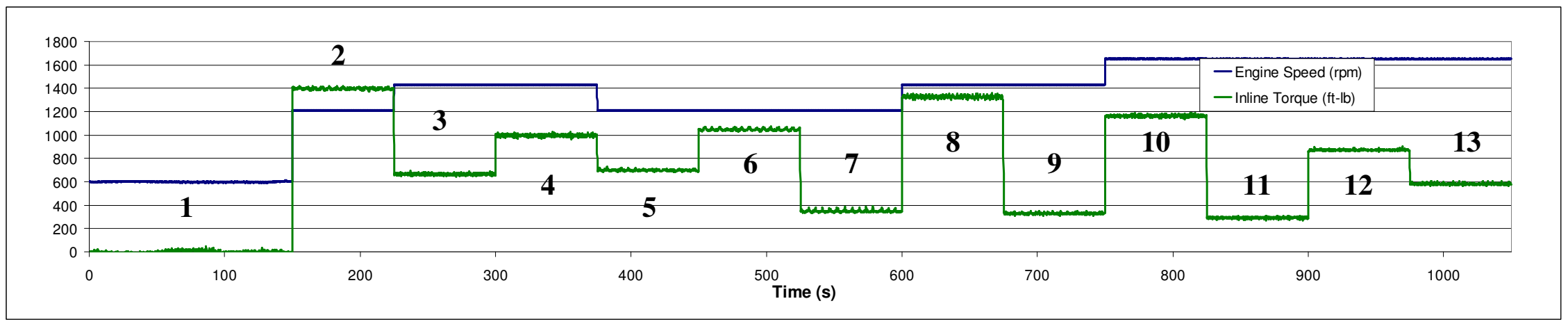

Figure 69 Engine Speeds and Torques of E01948_01 
The continuous PM data of E01948_01 is provided in Figure 70. It appears that the particle distributions and concentrations remained transient during every mode except 4, 12, and 13. For E01948_01 and all other SETs that were executed on the rebuilt DDC S60, the highest concentrations of accumulation mode particles occurred during mode 2, and the highest concentrations in nuclei mode particles occurred during mode 7 . The previously described relationships between PM concentrations and engine operating conditions were also present during SETs. Figure 70 shows continuous SET data, which includes the ramp segments that are missing from Figure 69. 


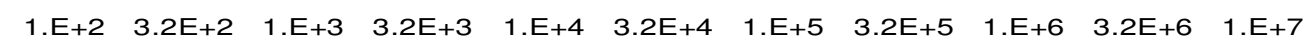
Spectral Density ((dN/dlogdp)/cc)

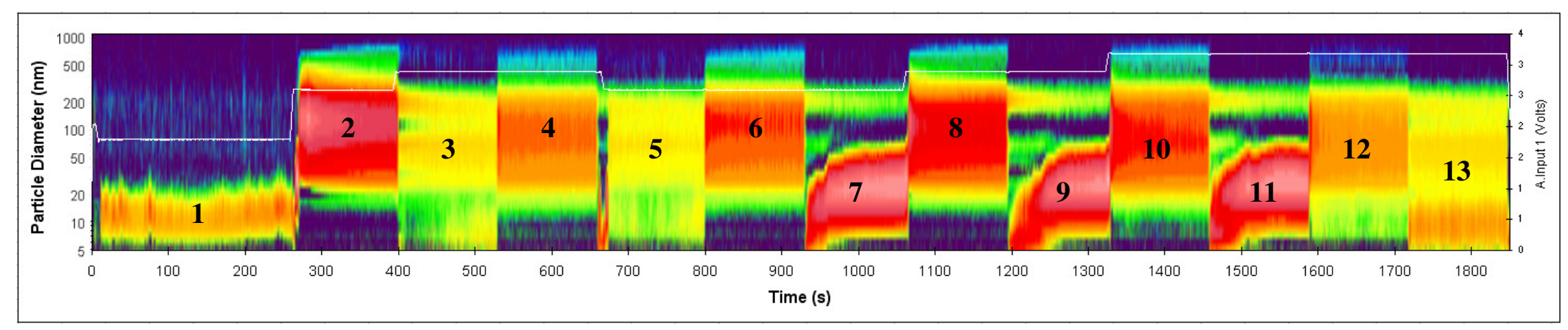

Figure 70 PM Concentrations During E01948_01 
The effects of fuel type, relative humidity, and dilution ratio on particle concentrations during steady-state conditions are discussed in the following sections. It was elected to study only six of the thirteen modes. Modes 2, 5, 6, 7, 10, and 11 were selected because they represent the boundaries of operating conditions during a SET. Mode 1 was ignored because of instability, which can be seen in Figure 70, of the nanoparticle concentrations. Figure 71, below, illustrates the engine speed and load setpoints of the six selected modes.

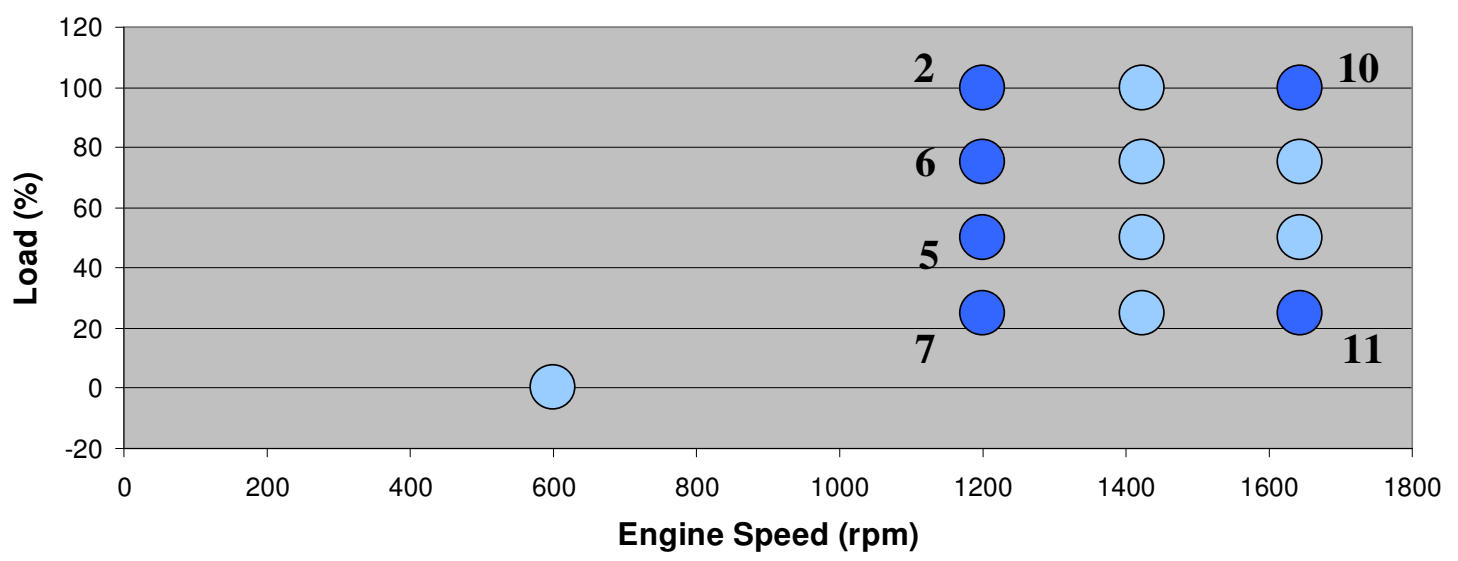

Figure 71 Speed/Load Domain of SETs

\subsubsection{Humidity Effects}

On the DDC S60, SETs were performed with the Guttman fuel on each relative humidity setting. The E02204 test sequence consisted of three repeat SET tests at 37\% relative humidity (within the primary dilution tunnel). Also consisting of three repeat SETs, the E02205 and E02206 test sequences were assessed at $\sim 88 \%$ and $\sim 60 \%$ relative humidity, respectively. The run-to-run COVs of each humidity-specific test sequence were separated by mode. Figure 72 displays the run-to-run COVs for mode 2 of the "high humidity" (E02205), "medium humidity" (E02206), and "low humidity" (E02204) test sequences. The medium humidity setting appears to have had significantly less 
variation (up to $60 \%$ less) between $649 \mathrm{~nm}$ and $866 \mathrm{~nm}$ than the high and low settings. Otherwise, the level of relative humidity didn't seem to affect the repeatability of the fuel and engine during mode 2. On behalf of the engine operating parameters of each mode, repeatability of the particle concentration measurements was considered acceptable wherever the COV was less than $5 \%$ for all three humidity settings. For mode 2 , the set points were $1199 \mathrm{rpm}$ and $1418 \mathrm{ft}-\mathrm{lb}$, and the run-to-run repeatability was acceptable between $27 \mathrm{~nm}$ and $487 \mathrm{~nm}$.

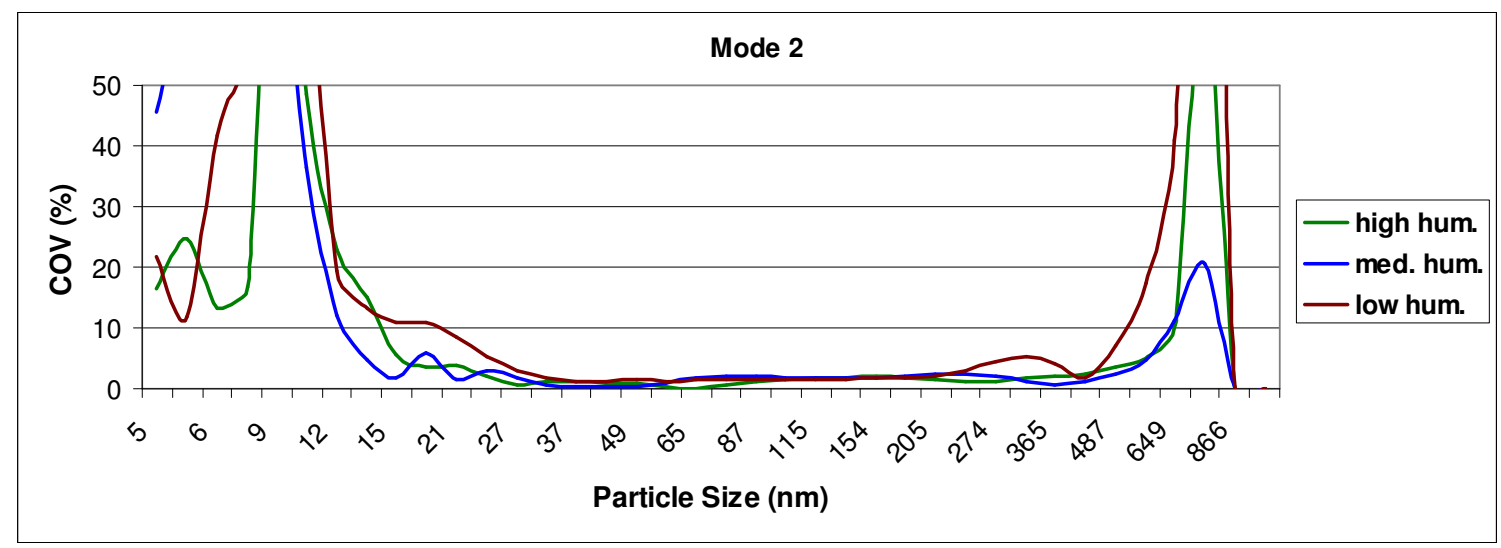

Figure 72 Run-to-Run Variation for Mode 2 of the E02204, E02205, and E02206 Test Sequences

The high, medium, and low humidity run-to-run COVs of mode 5 are shown in Figure 73. As in mode 2, the COV curves of mode 5 are similar in shape but have differences in magnitude (up to $50 \%$ ) outside of the "acceptable" range. The run-to-run repeatability for mode 5 was acceptable between $37 \mathrm{~nm}$ and $154 \mathrm{~nm}$. The engine was operated at approximately $1199 \mathrm{rpm}$ and $709 \mathrm{ft}-\mathrm{lb}$ during mode 5. 


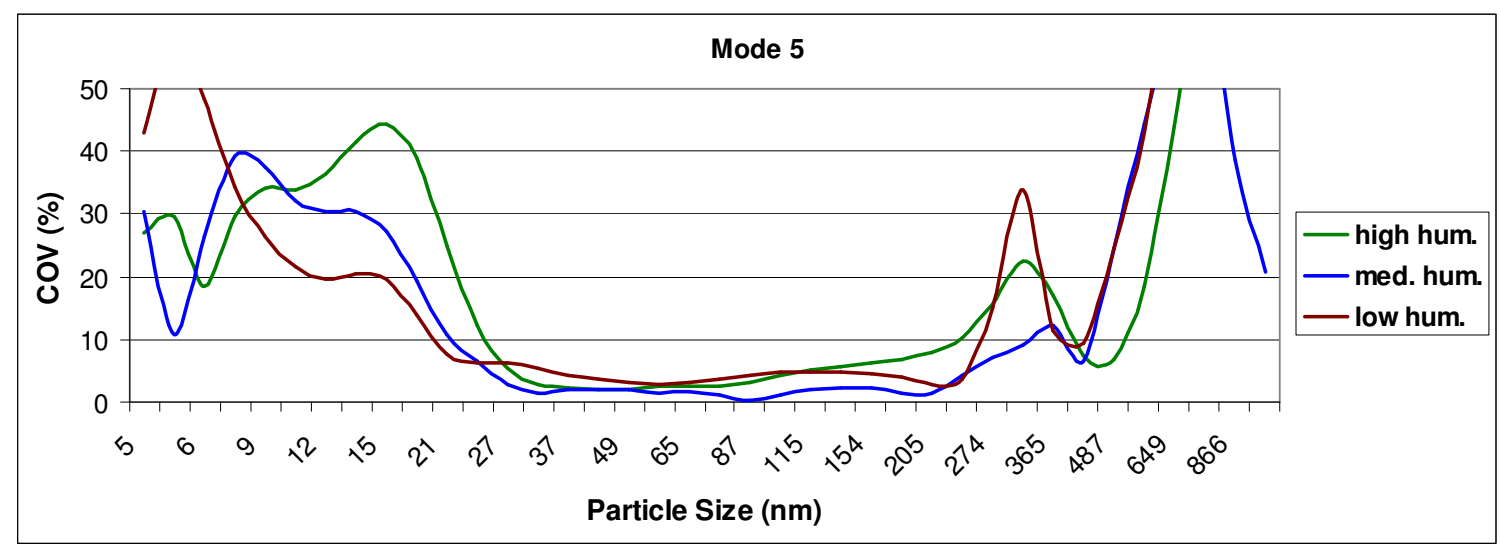

Figure 73 Run-to-Run Variation for Mode 5 of the E02204, E02205, and E02206 Test Sequences

Run-to-run variation for the humidity-specific testing of mode 6 can be found below in Figure 74. The engine operating conditions, $1199 \mathrm{rpm}$ and $1064 \mathrm{ft}-\mathrm{lb}$, were very close to the operating conditions of mode 2. As a result, the COV values for the three humidity settings of mode 6 were similar to those of mode 2; whereas the acceptable range was between $18 \mathrm{~nm}$ and $365 \mathrm{~nm}$, and the magnitudes appeared to be randomly variable below $15 \mathrm{~nm}$ and above $650 \mathrm{~nm}$.

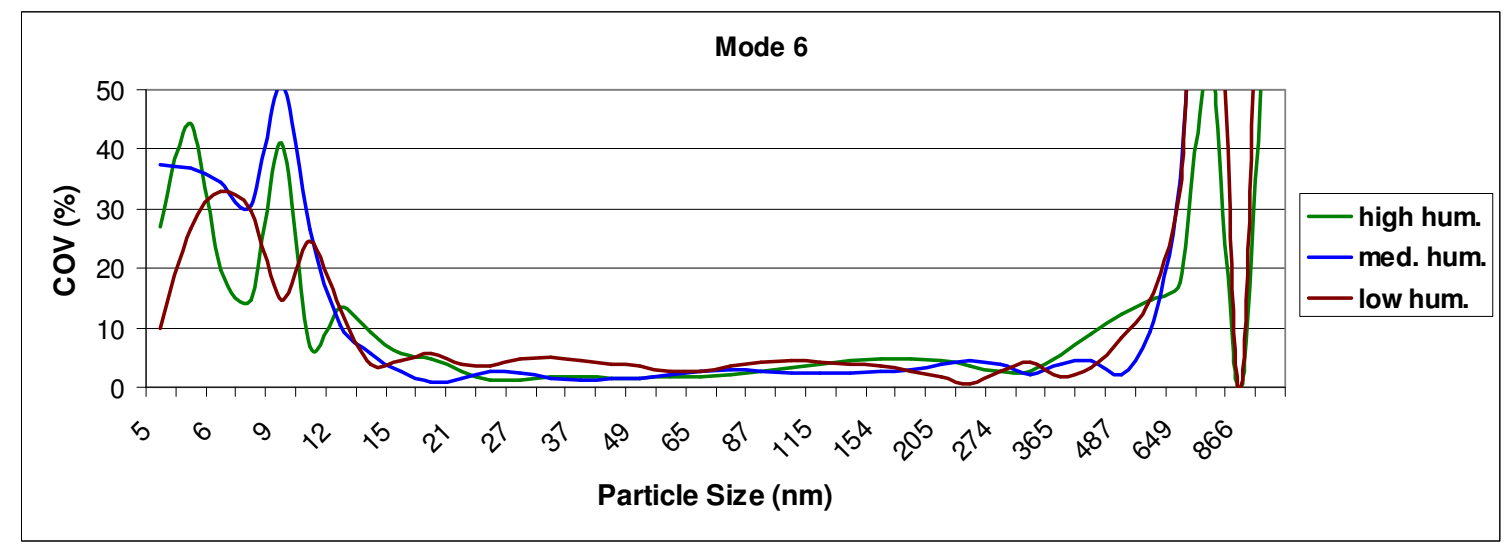

Figure 74 Run-to-Run Variation for Mode 6 of the E02204, E02205, and E02206 Test Sequences

Mode 7 was one of relatively low torque (355 ft-lb) for the given speed (1199 rpm). The run-to-run COV curves of Mode 7, shown in Figure 75, were very dissimilar 
among the three humidity settings. This could be partly due to the differences in humidity; but since the variation was normally greater than $20 \%$ and only three repeat tests were completed per humidity setting, it is impossible to make a valid claim. The acceptable COV values of mode 7 were in the range of $15 \mathrm{~nm}$ and $21 \mathrm{~nm}$.

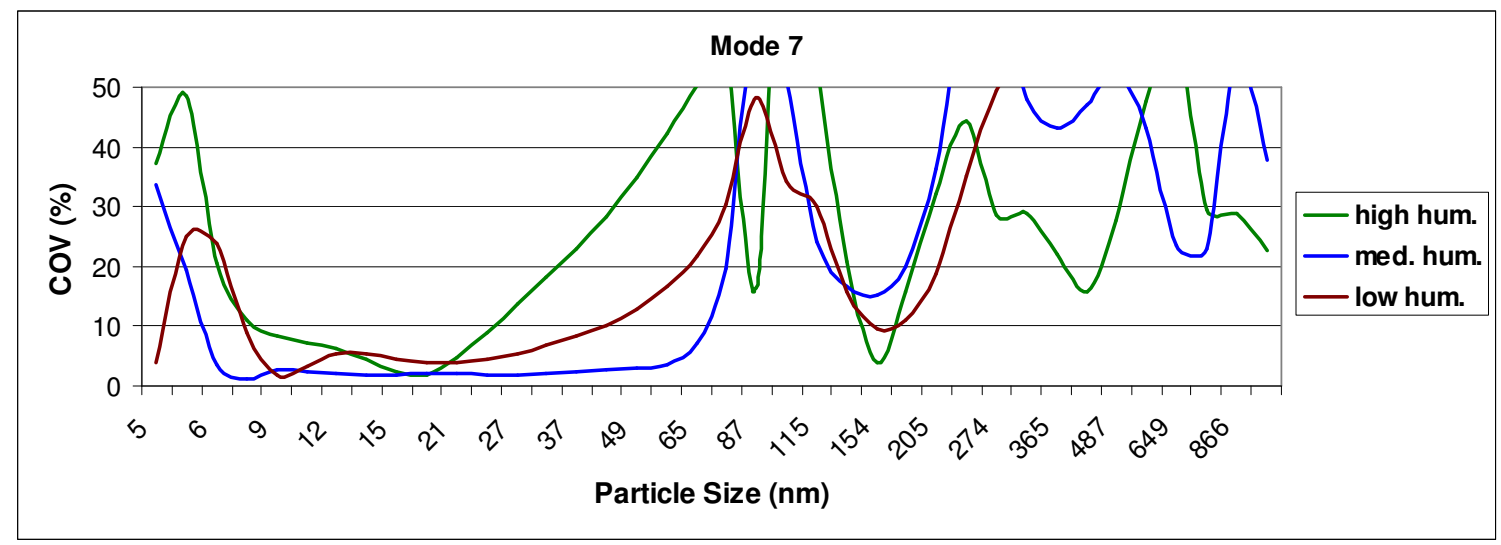

Figure 75 Run-to-Run Variation for Mode 7 of the E02204, E02205, and E02206 Test Sequences

Mode 10 indicated the highest power operation (369 hp) of any mode. It could be expected that the highest power mode would produce the least run-to-run variation, since more particle sizes would have concentrations above the detectible limit due to the overall dilution ratio being at its lowest. The COV curves of Mode 10 can be seen in Figure 76. Mode 10 did have the least run-to-run variation, and the engine speed and torque set points were $1644 \mathrm{rpm}$ and $1179 \mathrm{ft}-\mathrm{lb}$. The three humidity settings provided run-to-run COV curves of a consistent pattern. The COV curves peaked around $9 \mathrm{~nm}$, were acceptable between $24 \mathrm{~nm}$ and $487 \mathrm{~nm}$, and peaked again at about $866 \mathrm{~nm}$. 


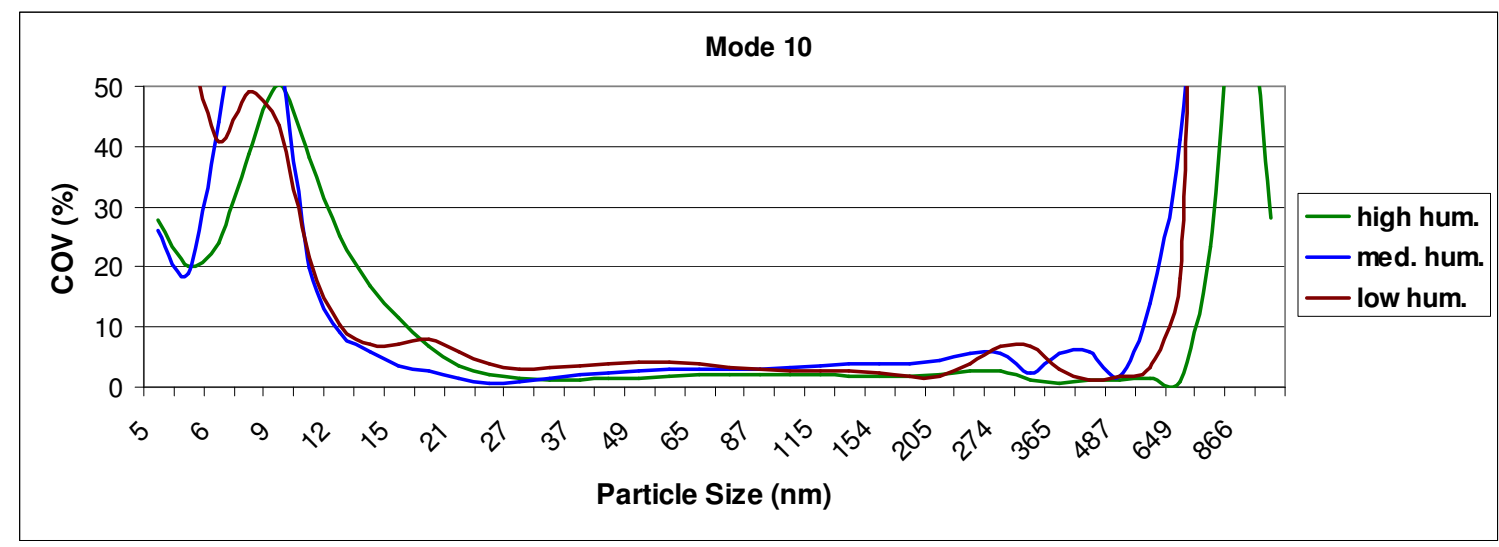

Figure 76 Run-to-Run Variation for Mode 10 of the E02204, E02205, and E02206 Test Sequences

The operating parameters of mode 11 were similar to those of mode 7 , and as seen in Figure 77 below, so were the run-to-run COV values. Mode 11 consisted of a high engine speed $(1644 \mathrm{rpm})$ and a low torque $(295 \mathrm{ft}-\mathrm{lb})$. The acceptable run-to-run variations were between $15 \mathrm{~nm}$ and $21 \mathrm{~nm}$, as in mode 7 . The variations appeared to be random for the particles larger than $87 \mathrm{~nm}$, and the magnitudes of variation were excessive (greater than $20 \%$ ) below $6 \mathrm{~nm}$ and above $37 \mathrm{~nm}$. The distinct dip in the COV curves (between $115 \mathrm{~nm}$ and $274 \mathrm{~nm}$ ) of both mode 7 and mode 11 was either caused by consistent noise on the electrometer rings due to soot buildup, artifacts of the previous modes, or a steady emission of accumulation mode particles.

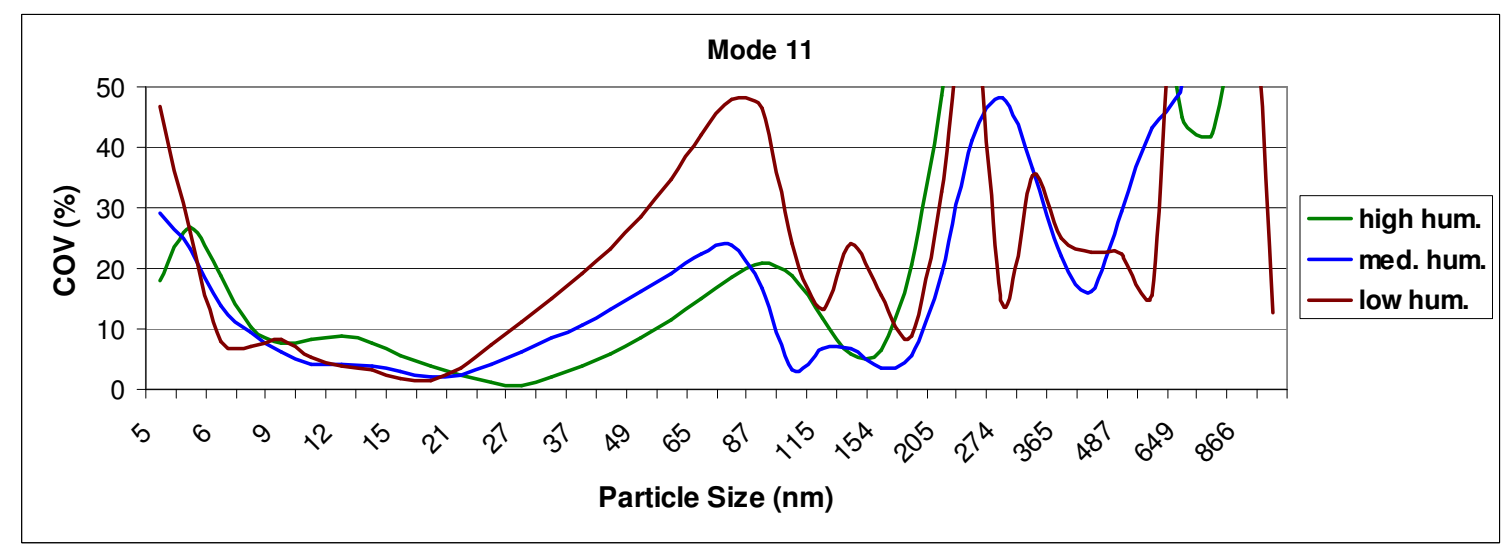

Figure 77 Run-to-Run Variation for Mode 11 of the E02204, E02205, and E02206 Test Sequences 
Regardless of the level of humidity, the COV values were basically consistent for each mode, meaning that run-to-run repeatability is governed more solidly by engine operating conditions than by relative humidity within the primary dilution tunnel. If humidity had any effect on run-to-run repeatability, it was only during periods of high speed and low load engine operation. Most of the steady state particle distributions were unimodal, meaning that only one particle size range had detectable aerosol concentrations. Much of the variation between repeat tests was due to low concentrations of particles that were smaller or larger than those of the unimodal distributions. Some run-to-run variation was probably caused by the inability of aerosol concentrations to stabilize during the two minute window of each mode. Most likely, the concentrations failed to stabilize because the temperatures of the lube oil and crank case were still rising or falling. For the three humidity settings, the average COV scores of modes 2, 5, 6, 7, 10, and 11 were $210,331,221,613,206$, and 587, respectively. Characterized by COV scores, the run-to-run variation of each mode can be loosely related to overall dilution ratio. The overall dilution ratios, on average, were $97,149,115,186,64$, and 131, respectively, for modes $2,5,6,7,10$, and 11 . With the exception of modes 5 and 6 , the ranking order of average modal COV scores matched the ranking order of overall dilution ratio.

There was no repeat testing for the fuel additive and dilution ratio-specific SETs. Since the same fuel and engine were used in the steady state analyses of relative humidity, fuel additives, and dilution ratio, the particle size ranges of each mode that had acceptable run-to-run COV values (from the humidity-specific results) were applied to the analysis of fuel type and dilution ratio. 
Significant differences were determined between the high, medium, and low humidity test results using the same technique (if sum of standard deviations is less than difference, difference is significant) as in the FTP Results section. The particle concentrations for high (88\%) and low (37\%) relative humidity were compared to those of medium (60\%) relative humidity. The significant differences between the average particle concentrations during the last 10 seconds of mode 2 are illustrated by Figure 78. The light and dark blue bars represent the concentration differences between low humidity and medium humidity. The yellow and dark orange bars represent differences between high humidity and medium humidity. The dark colored bars signify that the percent differences are significant. As depicted by Figure 78, the high humidity setting resulted in a $2 \%$ to $10 \%$ decrease in particles between $15 \mathrm{~nm}$ and $87 \mathrm{~nm}$ and a $6 \%$ to 11 $\%$ increase between $422 \mathrm{~nm}$ and $562 \mathrm{~nm}$. The low humidity setting provided a $9 \%$ to 18 $\%$ increase between $422 \mathrm{~nm}$ and $487 \mathrm{~nm}$. No gravimetric PM measurements were made, but it is estimated that during mode 2 , the humidity setting had little to no effect on the mass-based PM emissions. 


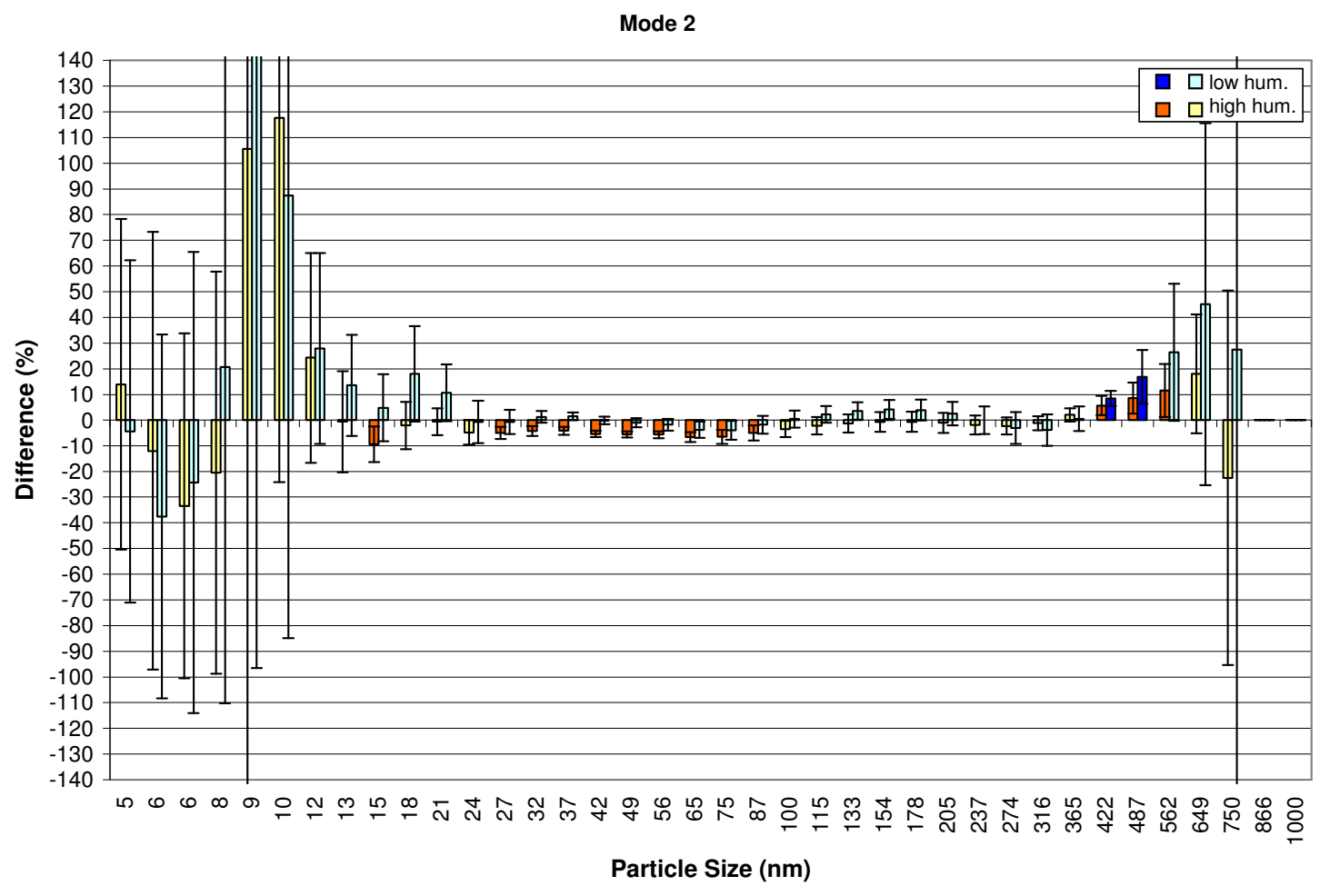

\section{Figure 78 Differences in Particle Concentrations of Mode 2 Due to Humidity Change}

For the humidity test results of mode 5, the percent differences between particle concentrations can be found in Figure 79. During the engine operating conditions of mode 5 , the high humidity and low humidity settings provided very similar results. They each resulted in a concentration decrease of less than $10 \%$ from $32 \mathrm{~nm}$ to $100 \mathrm{~nm}$, although only the high humidity differences were significant. In the $205 \mathrm{~nm}$ to $487 \mathrm{~nm}$ size range, they both caused reductions in particle concentrations of between $10 \%$ and 67 $\%$. 


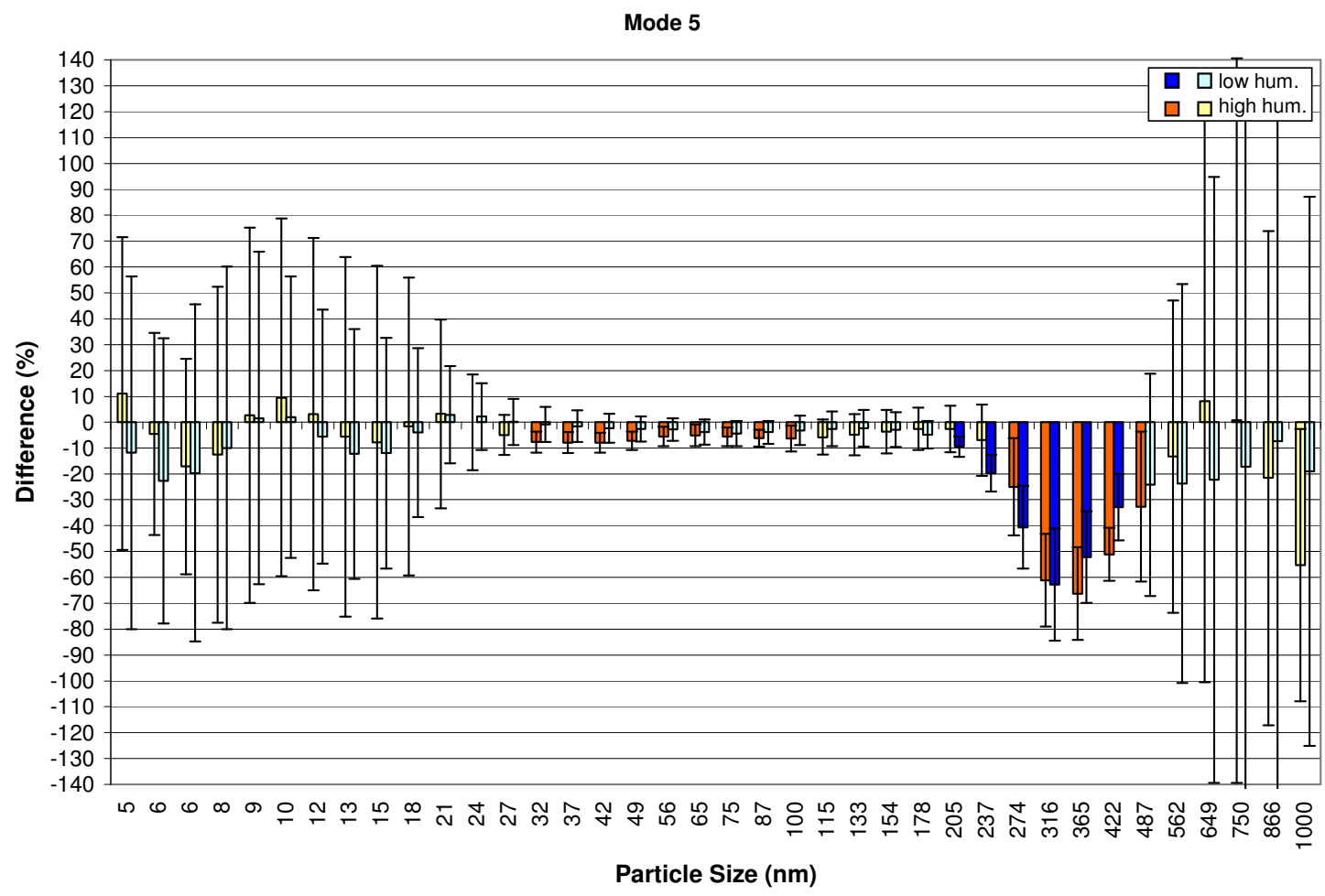

\section{Figure 79 Differences in Particle Concentrations of Mode 5 Due to Humidity Change}

During mode 6 , the only particle concentrations of the low humidity setting that showed significant difference from those of the medium humidity setting were in the 274 $\mathrm{nm}$ to $316 \mathrm{~nm}$ size range. As seen in Figure 80, the maximum significant difference for the low humidity particles of mode 6 was a reduction of about $9 \%$. There were significant concentration reductions, which were anywhere from $4 \%$ to $10 \%$, caused by the high humidity setting between $15 \mathrm{~nm}$ and $75 \mathrm{~nm}$. Of the six modes that were analyzed, mode 6 nearly demonstrated the least and lowest significant differences between humidity settings. 


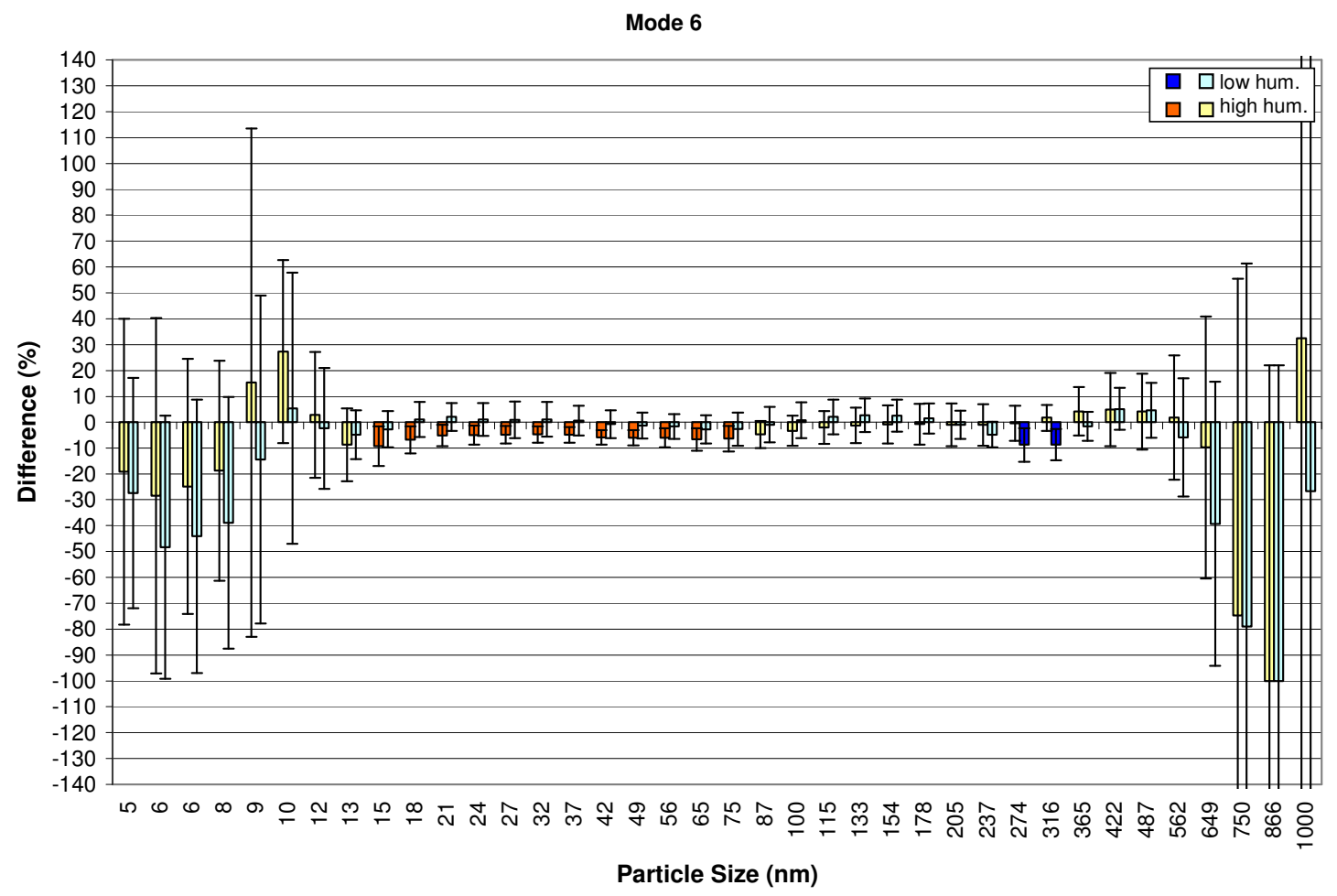

Figure 80 Differences in Particle Concentrations of
Mode 6 Due to Humidity Change

Regardless of the fact that the highest run-to-run variation belonged to mode 7 , significant differences were still present, as illustrated by Figure 81, between the low and medium humidity settings. In the $7 \mathrm{~nm}$ to $10 \mathrm{~nm}$ range, the low humidity tests resulted in particle concentration reductions of $8 \%$ to $24 \%$. Some of these reductions were close to being considered insignificant, because the error bars were within a few percent of being greater in magnitude than the differences. During mode 7, low relative humidity caused the greatest single significant difference in particle concentration (increase of $120 \%$ at 75 $\mathrm{nm}$ ) of the humidity test results. Between $24 \mathrm{~nm}$ and $75 \mathrm{~nm}$, the low humidity setting was responsible for rises in particle concentrations of $9 \%$ to $120 \%$. The average PM concentration values of the high humidity setting were not significantly different from those of medium humidity because of the amount of run-to-run variation. It is apparent 
in Figure 81, however, that the high and low humidity settings resulted in similar increases or decreases in concentration.

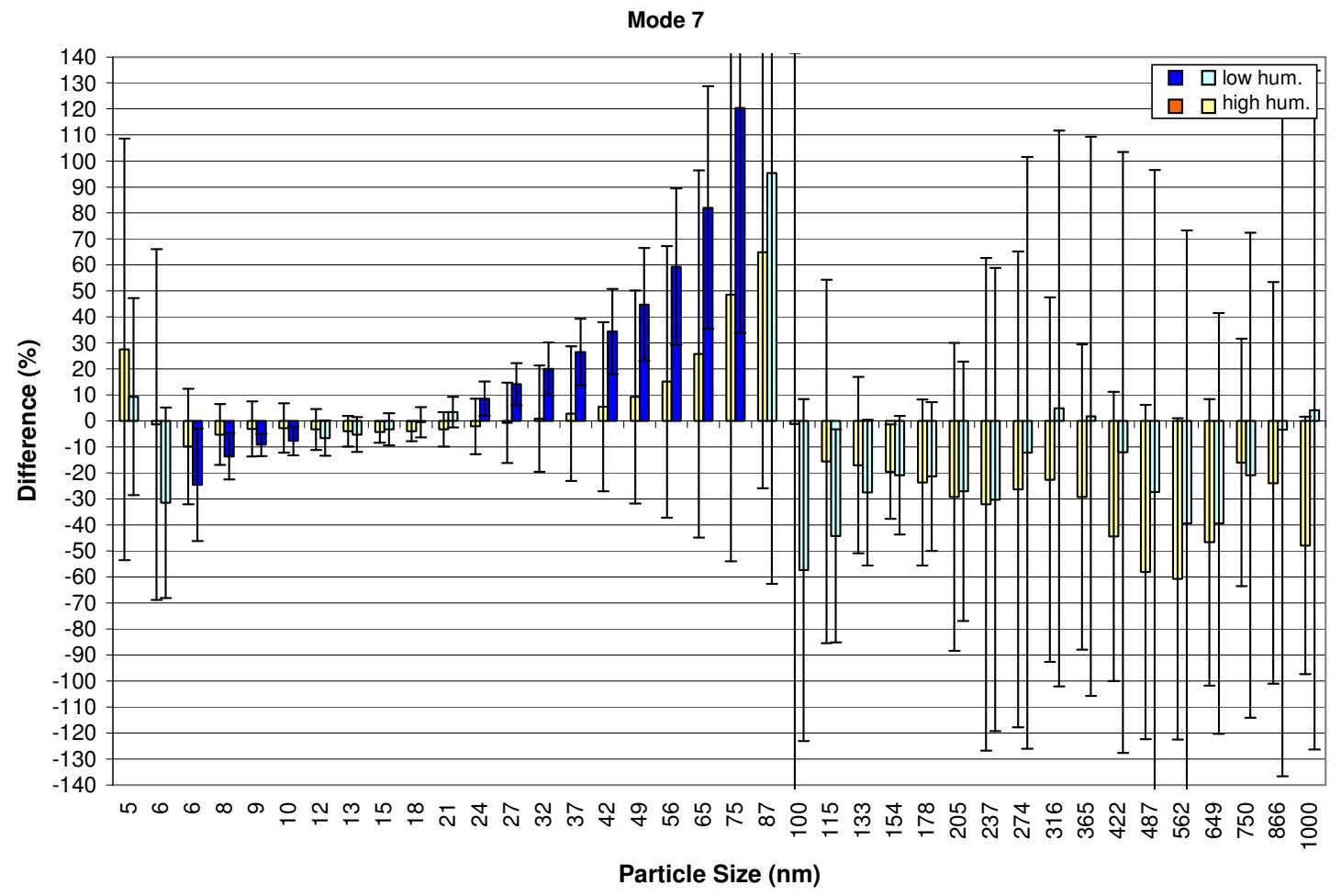

Figure 81 Differences in Particle Concentrations of Mode 7 Due to Humidity Change

Figure 82 illustrates the particle concentration differences due to changes in humidity. Of all analyzed modes, mode 10 provided significant differences for the fewest particle sizes. Furthermore, the greatest significant difference (7\%) of mode 10 is the lowest maximum significant difference for the humidity-specific particle concentrations of any mode. While the low humidity testing resulted in no significant differences in concentration for mode 10 , significant reductions of $4 \%$ to $7 \%$ in the 27 $\mathrm{nm}$ to $75 \mathrm{~nm}$ range were attributed to the high humidity setting. 


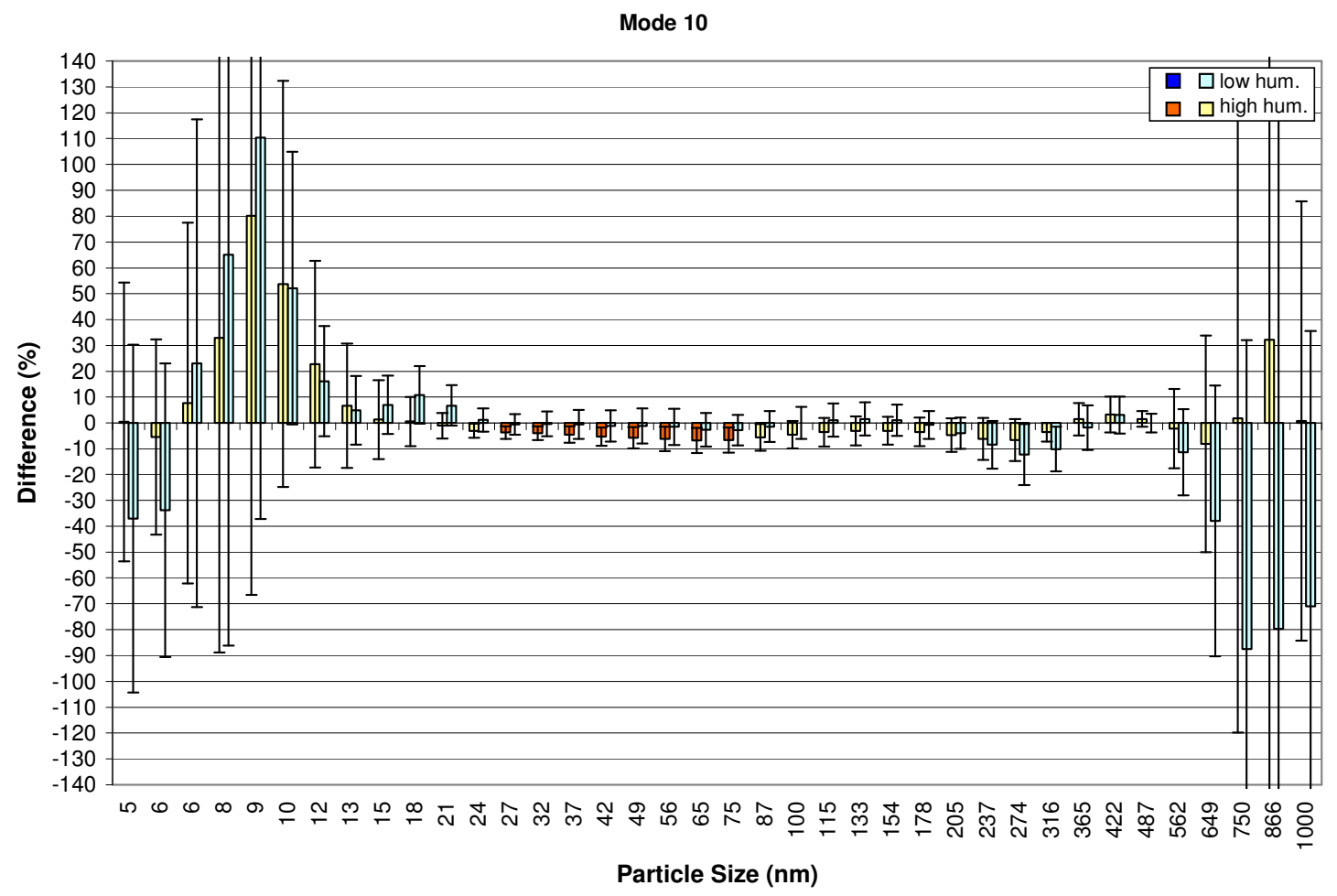

\section{Figure 82 Differences in Particle Concentrations of Mode 10 Due to Humidity Change}

Like mode 7, mode 11 was afflicted by high run-to-run variation (average COV score of 587), but there were still significant differences between the PM concentrations of the high, medium, and low humidity settings. Figure 83 illustrates that during mode 11 , the low humidity setting was responsible for a $22 \%$ to $70 \%$ decrease in some of the nuclei mode particles and a $23 \%$ to $83 \%$ decrease in accumulation mode particles between $154 \mathrm{~nm}$ and $274 \mathrm{~nm}$. Increases in particle concentration for the $24 \mathrm{~nm}$ to $100 \mathrm{~nm}$ size range were anywhere from $9 \%$ to $88 \%$, and they were due to high relative humidity. From $133 \mathrm{~nm}$ to $154 \mathrm{~nm}$, the high humidity setting caused reductions in concentration of $12 \%$ to $15 \%$. As explained previously, the engine speed and load set points of mode 11 were similar to those of mode 7. The significant differences that precipitated from mode 11, however, were nothing like those described for mode 7. This is because the run-to- 
run variations of modes 7 and 11 were not exactly the same. Ignoring error bars, the extreme humidity settings of modes 7 and 11 both resulted in increasing particle concentration reductions from $15 \mathrm{~nm}$ to $6 \mathrm{~nm}$ and increasing particle concentration gains from $18 \mathrm{~nm}$ to $75 \mathrm{~nm}$. In such a manner, the humidity test results of modes 7 and 11 were similar.

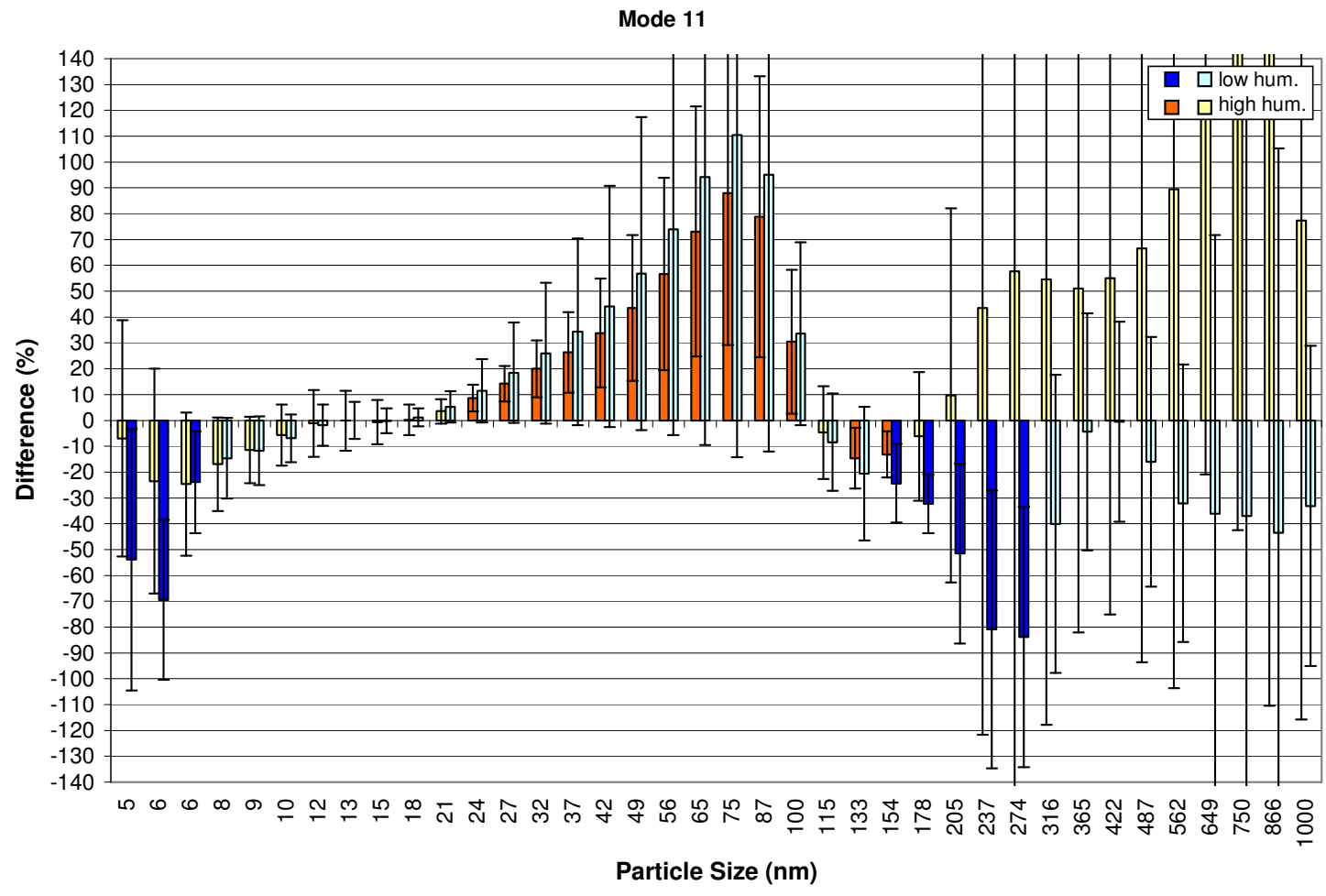

\section{Figure 83 Differences in Particle Concentrations of Mode 11 Due to Humidity Change}

In the humidity results of modes $2,5,6,7,10$, and 11 , the significant percent differences in particle concentration were based on only three repeat tests per humidity setting. For this reason and the fact that run-to-run variation was typically high (COV scores of 206 to 613), the accuracy of the differences is statistically unknown. It is impossible, with the collected data, to conclude which humidity-caused differences can be repeated and which ones were by chance. It appears that humidity change probably 
only altered the particle concentrations enough to have an effect on the brake-specific TPM during modes 5,7 , and 11 . This means that relative humidity within the dilution tunnel effects PM emissions of the Guttman/rebuilt DDC S60 only during low load $(<50$ $\%)$ operation. The average particle concentrations for each mode $(2,5,6,7,10$, and 11) and each humidity setting $(37 \%, 88 \%$, and $60 \%)$ can be found in the Humidity Effects section of the Appendix.

MATLAB ANOVA was explored as an alternate statistical method of determining which PM concentration differences were significant. As examples, all of the steady state humidity test results were analyzed with ANOVA. Figure 84 indicates which particle concentrations of mode 2 were significantly different, according to ANOVA, between humidity settings. In Figure 84, there are three circles with lines through them (one for each humidity setting) for each particle size. The circles give a rough indication of brake-specific particle count, and the line lengths are related to runto-run variation. The sets of circles with blue horizontal lines represent the particle sizes whose concentrations were significantly affected by humidity. According to ANOVA, the range of sizes for mode 2 where differences were determined to be significant was 65 $\mathrm{nm}$ to $205 \mathrm{~nm}$. The method described above that incorporates equation 11 indicated that for mode 2, the changes in humidity resulted in significant differences at $15 \mathrm{~nm}$, from 27 $\mathrm{nm}$ to $87 \mathrm{~nm}$, and between $422 \mathrm{~nm}$ and $562 \mathrm{~nm}$. The ANOVA results of mode 2, as opposed to the ANOVA results of modes 3 through 13, indicated the highest number of particle sizes that had significant differences. 


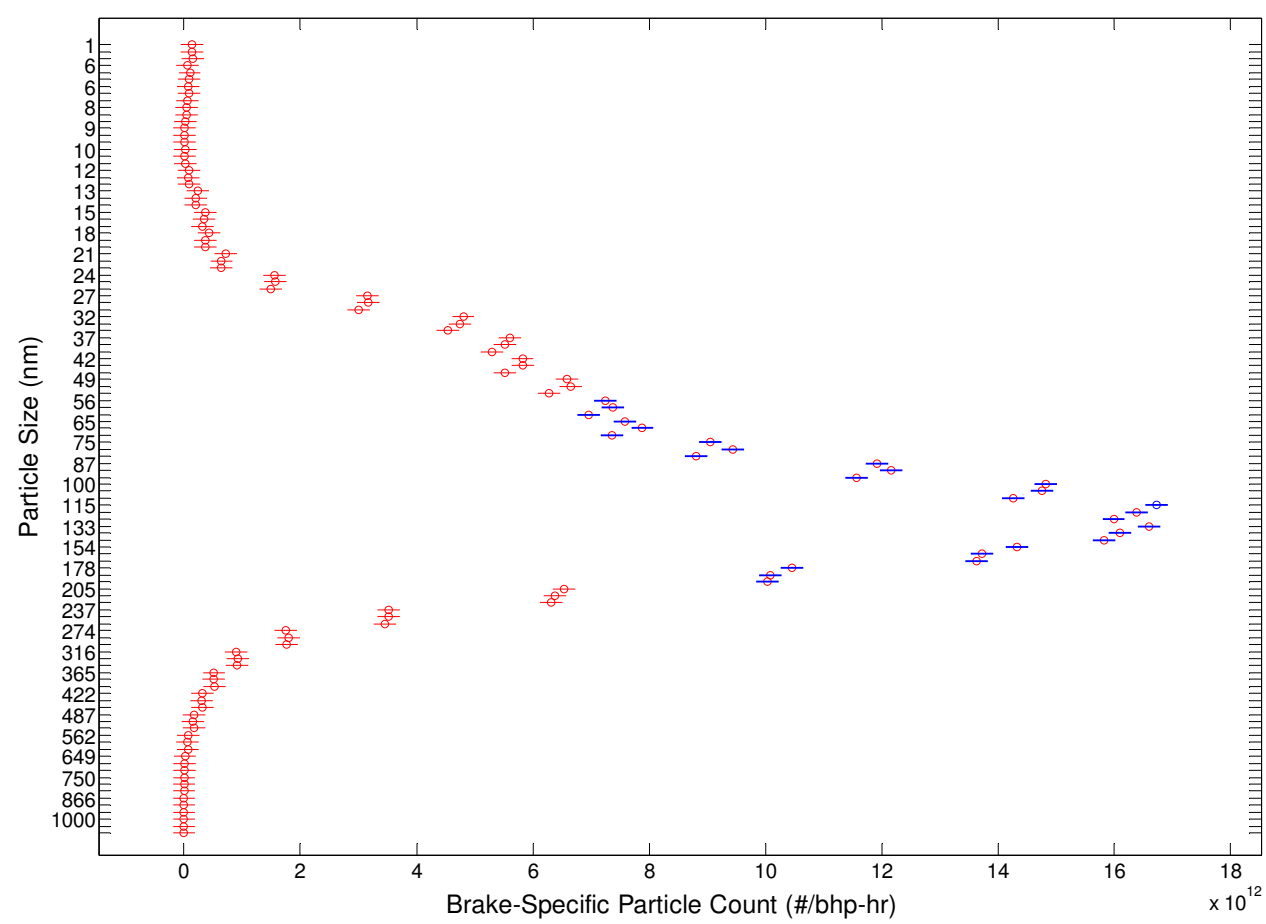

Figure 84 Particle Sizes that Have Significantly Different Average Values for Mode 2 Due to Humidity Change (According to ANOVA)

As can be seen in Figure 85, the ANOVA analysis of mode 13 specified that there were no particle sizes that had significantly different concentrations due to humidity variation. The ANOVA analysis in MATLAB assumes the variances are equal for all particle sizes. That is, ANOVA only solves for a single variance from all particle sizes, and it uses that variance to determine significance. 


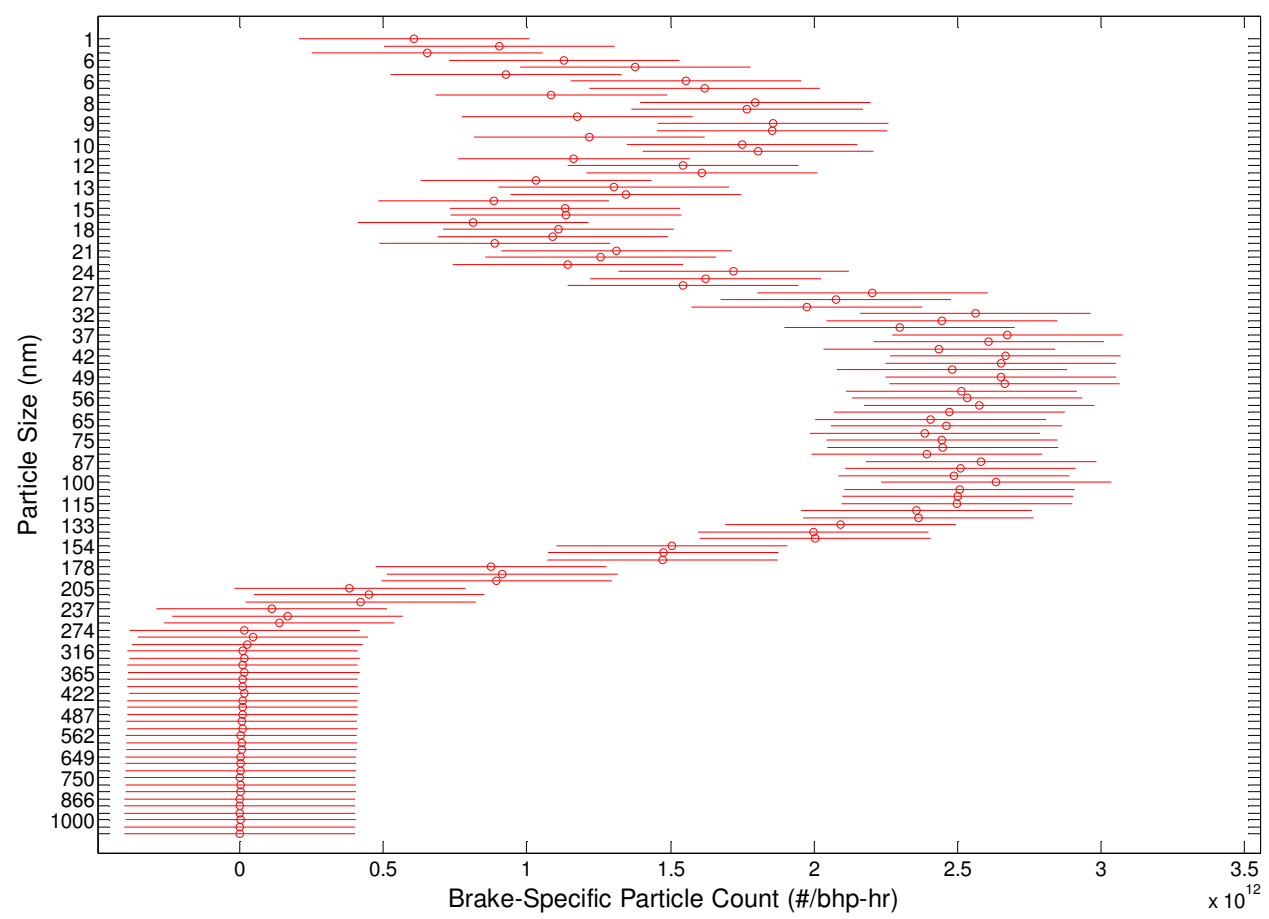

Figure 85 Particle Sizes that Have Significantly Different Average Values for Mode 13 Due to Humidity Change (According to ANOVA)

The use of MATLAB ANOVA illustrates which concentrations are significantly different and gives an indication of particle concentrations for each humidity setting, but fails to provide a means of determining percent differences. The ANOVA analyses of modes 3 through 12 are represented by Figures A-59 through A-67 in the Humidity Effects section of the Appendix. Immediately after Figure A-67, the MATLAB code for the ANOVA analyses is also provided in the Appendix. In every case where significance was determined by both methods (equation 11 and ANOVA), ANOVA yielded a fewer number of particle sizes with significant differences. This is because a calculation for 95 $\%$ certainty is incorporated into the ANOVA statistical model. Perhaps in an application where ten or more repeat tests were administered, the $95 \%$ certainty of ANOVA would be practical, but for only three repeat tests of each humidity setting, the results of 
ANOVA are questionable. In short, the statistics of ANOVA do not normally apply to data sets of this size. Therefore, the conclusions of this research were based on the technique developed and described above on page 81 .

\subsubsection{Fuel A vs. Fuel B}

The effects of fuel additives were studied over steady state conditions, where low cetane, low cetane B10A, and low cetane B20A were used with the rebuilt DDC S60. Only one SET per fuel was performed, and all three tests were completed on the same day. Test number E02138 corresponds to the low cetane SET, E02139 corresponds to the low cetane B10A SET, and E02141 identifies the low cetane B20A SET. In the following illustrations and discussion, low cetane was used as a standard with which to compare low cetane B10A and low cetane B20A. Since only one test per fuel was allowed, the exactness of percent difference comparisons was not applicable. Therefore, a general way of comparing results was developed. The apparent fuel effect factor (AFEF) was used to describe the differences in particle concentration that appeared to be caused by the use of fuel additives. The AFEF is given below by equation 18 .

$$
A F E F=\frac{C_{a}}{C_{b}} \quad \text { Equation } 18
$$

The concentration of a given particle size is denoted by $C$, where $C_{a}$ symbolizes the concentrations of the additized fuel and $C_{b}$ stands for the concentrations of the base fuel. For example, an AFEF of 1.1 indicates that the fuel additive appeared to be responsible for a $10 \%$ increase in particle concentration. An AFEF of 0.9 suggests a 10 $\%$ decrease in concentration. 
It was not possible to determine which particle sizes had acceptable run-to-run repeatability for each mode, since only one test per fuel was performed. Therefore, and because the rebuilt DDC was used for all SETs, applying the acceptable repeatability (5 $\%$ COV or less) ranges of the humidity test results helped to determine which particle sizes to compare for each mode. In each of the following six figures, the dark blue and orange columns indicate the AFEF for particle sizes that were projected to have had measurement precisions of $+-5 \%$. The dark and light blue columns correspond to the differences between low cetane and low cetane B10A, and the orange and yellow columns signify the differences between low cetane and low cetane B20A.

The AFEF values for the test results of mode 2 are present in Figure 86. It appears that the use of low cetane B10A over low cetane resulted in particle concentration increases of $1 \%$ to $14 \%$ between $13 \mathrm{~nm}$ and $27 \mathrm{~nm}$ and particle concentration reductions of $1 \%$ to $11 \%$ between $37 \mathrm{~nm}$ and $649 \mathrm{~nm}$. The effect of low cetane B20A was the same for every particle size, but the AFEF values were greater in magnitude. From $13 \mathrm{~nm}$ to $27 \mathrm{~nm}$ and $32 \mathrm{~nm}$ to $649 \mathrm{~nm}$, low cetane B20A caused PM concentration increases of between $8 \%$ and $30 \%$ and reductions of between $6 \%$ and 28 $\%$, respectively. 


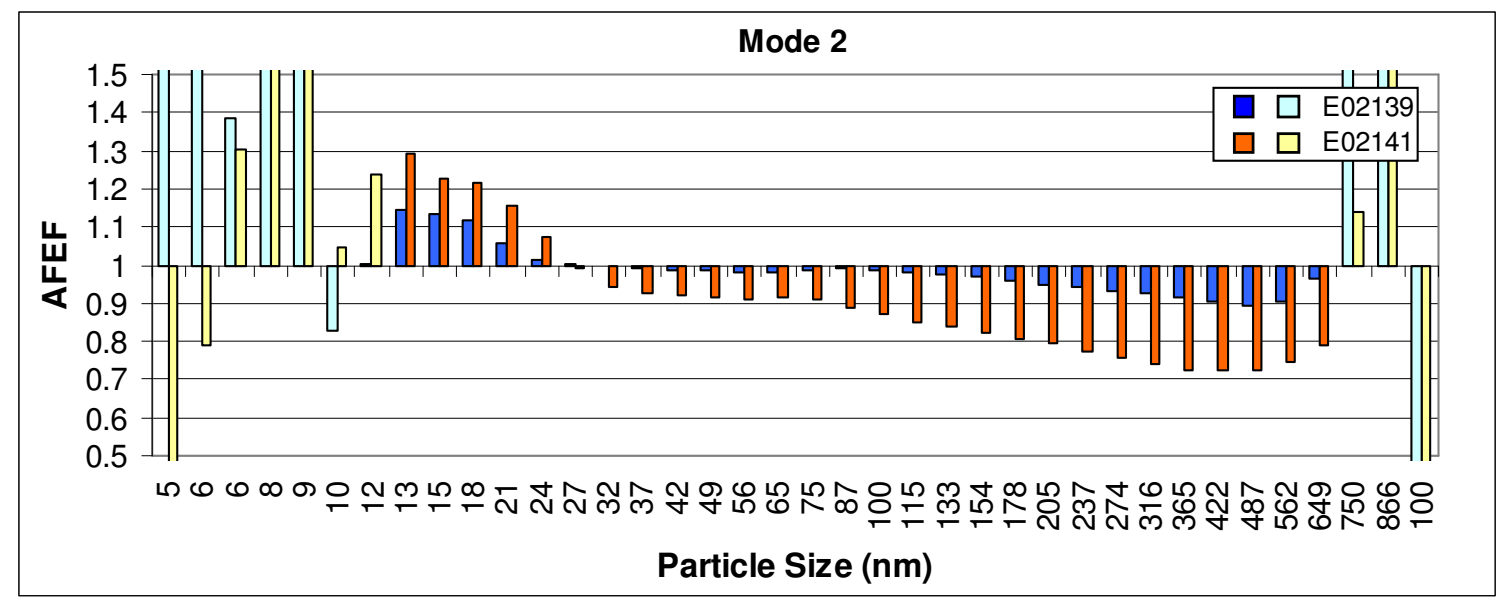

Figure 86 Apparent Fuel Effects of Biodiesel Addition to Low Cetane Diesel for Mode 2

For mode 5, the use of low cetane B20A once again provided concentration variations that were similar to but more prominent than those of low cetane B10A. As illustrated by Figure 87 , the use of a $10 \%$ biodiesel blend resulted in particle concentration reductions of $2 \%$ to $12 \%$ between $27 \mathrm{~nm}$ and $316 \mathrm{~nm}$. In the same particle size range, the $20 \%$ biodiesel blend caused reductions of $14 \%$ to $33 \%$. The engine operating parameters of mode 5 provided unique results in that there were no increases in the particle concentrations of any size due to the incorporation of biodiesel. 


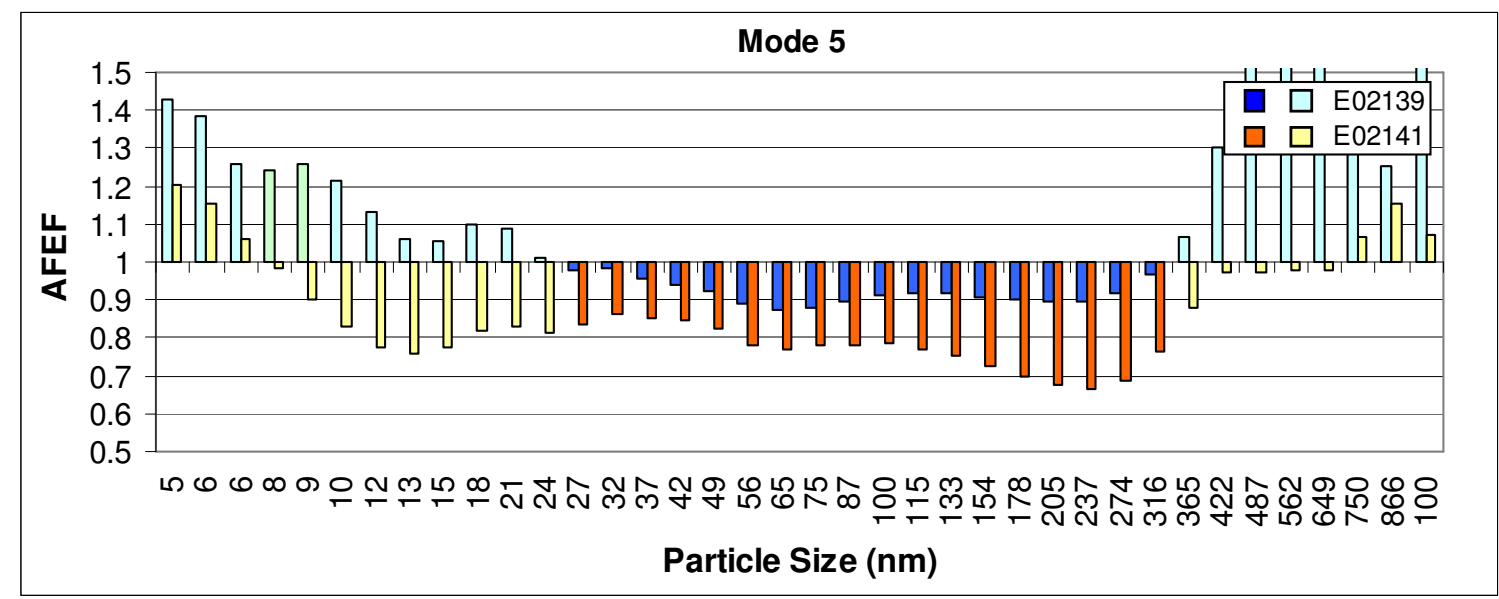

Figure 87 Apparent Fuel Effects of Biodiesel Addition to Low Cetane Diesel for Mode 5

The apparent fuel effect results of mode 6 are illustrated below by Figure 88 . The use of low cetane B10A over the base fuel resulted in concentration increases of $2 \%$ to $13 \%$ in the $12 \mathrm{~nm}$ to $24 \mathrm{~nm}$ range and $1 \%$ to $20 \%$ reductions in the $27 \mathrm{~nm}$ to $649 \mathrm{~nm}$ size range. Similarly, particle concentration increases of $2 \%$ to $17 \%$ between $12 \mathrm{~nm}$ and $21 \mathrm{~nm}$ and concentration reductions of $4 \%$ to $49 \%$ between $27 \mathrm{~nm}$ and $649 \mathrm{~nm}$ were attributed to the use of low cetane B20A over low cetane.

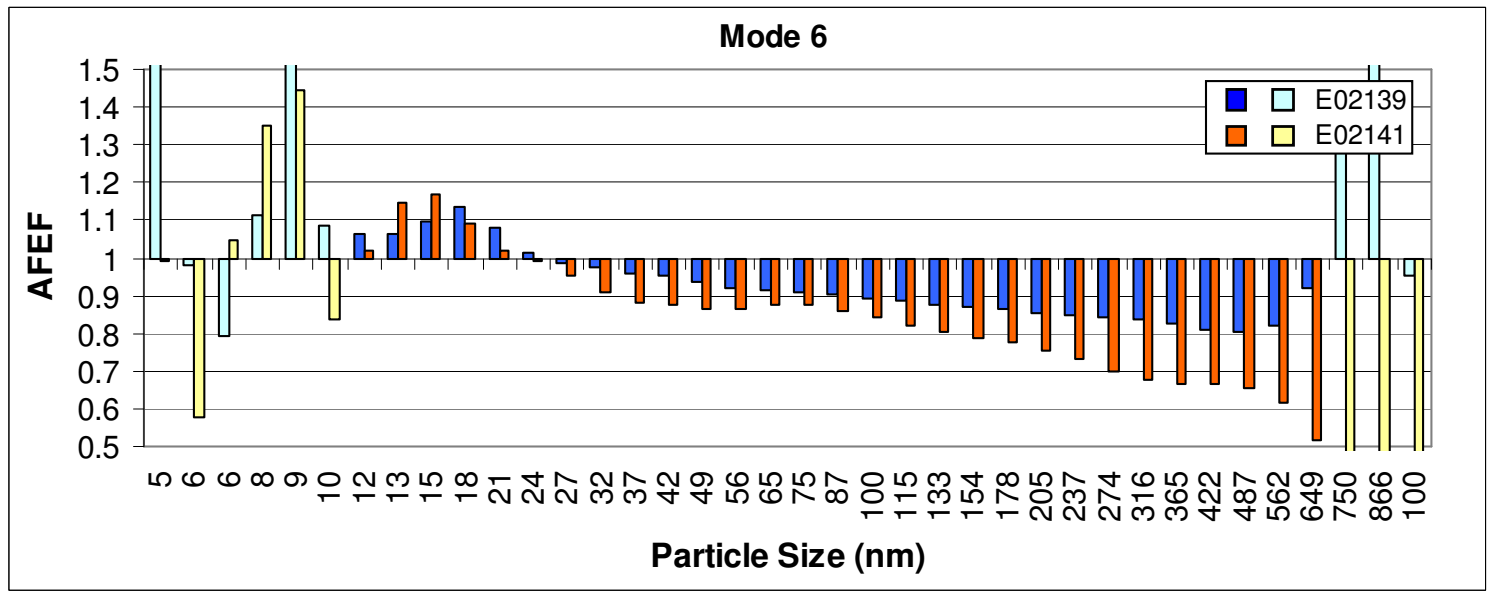

Figure 88 Apparent Fuel Effects of Biodiesel Addition to Low Cetane Diesel for Mode 6 
For mode 7, the AFEF values are given below by Figure 89 . The indicated effects of fuel properties during mode 7 resembled the effects of altering relative humidity within the dilution tunnel. Both additives caused an increasing reduction of concentrations between $15 \mathrm{~nm}$ and $7 \mathrm{~nm}$; and both were responsible for an increasing gain in particle concentrations between $24 \mathrm{~nm}$ and $56 \mathrm{~nm}$. For both additized fuels, the reductions in nuclei mode particles of mode 7 were between $1 \%$ and $14 \%$ and the gains in accumulation mode particles were between $3 \%$ and $36 \%$.

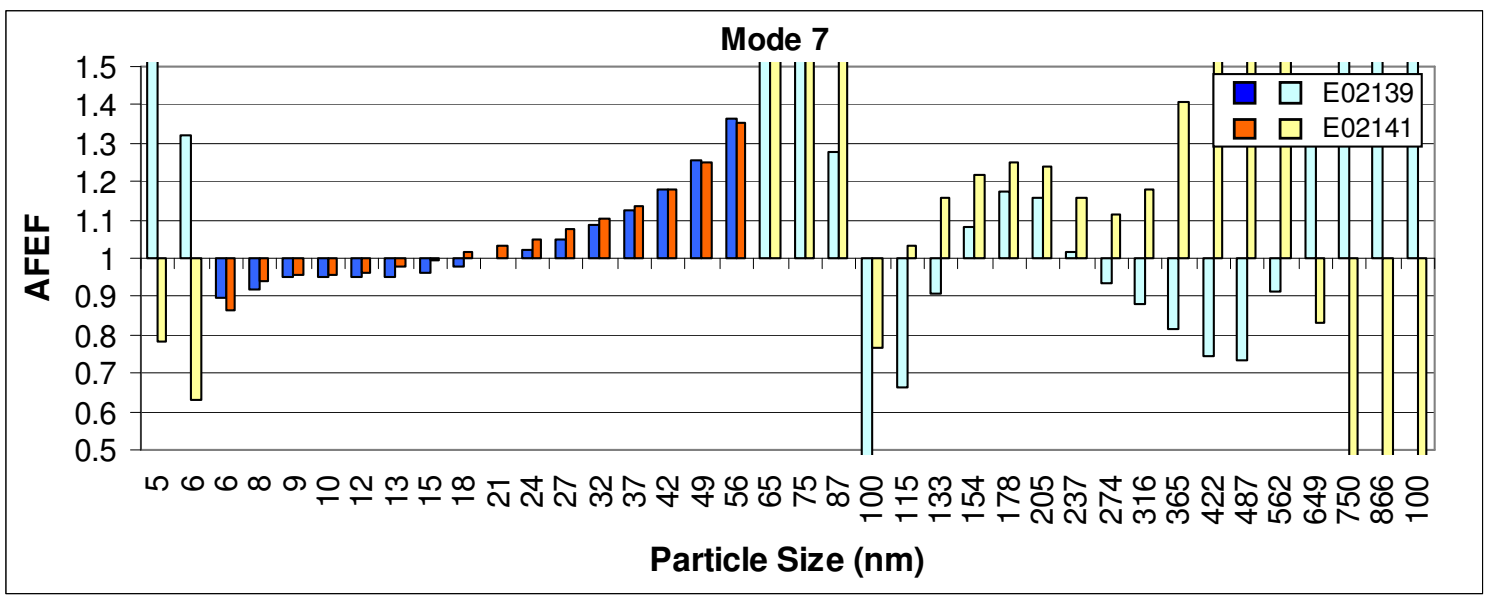

Figure 89 Apparent Fuel Effects of Biodiesel Addition to Low Cetane Diesel for Mode 7

The differences between fuels are shown for mode 10 by the AFEF values of Figure 90 . In the $15 \mathrm{~nm}$ to $21 \mathrm{~nm}$ particle size range, it was evident that the $\mathrm{B} 10 \mathrm{~A}$ and B20A blends caused concentration increases of $3 \%$ to $6 \%$, and $1 \%$ to $4 \%$, respectively. Between $37 \mathrm{~nm}$ and $487 \mathrm{~nm}$, the effect of low cetane B20A appeared to once again be the same as that of low cetane $\mathrm{B} 10 \mathrm{~A}$, only greater. In this range, it was rationalized that the use of the $10 \%$ biodiesel blend caused particle concentration reductions of $1 \%$ to $16 \%$, and the use of the $20 \%$ blend resulted in reductions of $2 \%$ to $24 \%$. 


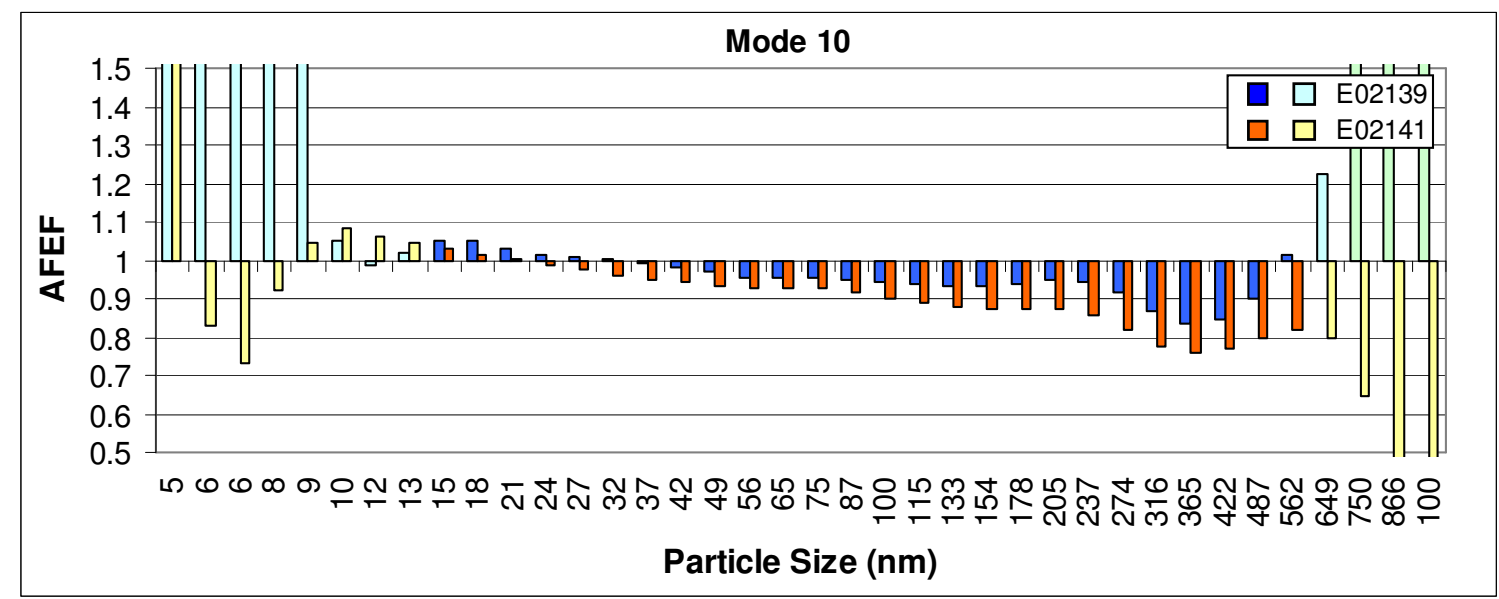

Figure 90 Apparent Fuel Effects of Biodiesel Addition to Low Cetane Diesel for Mode 10

Mode 11 was the only case in which the AFEF trends of each biodiesel additive were not the same. As illustrated by Figure 91, the AFEF trend of low cetane B10A was a continuous decrease from $9 \mathrm{~nm}$ to $37 \mathrm{~nm}$. The AFEF trend of low cetane B20A was a continous increase from $9 \mathrm{~nm}$ to $37 \mathrm{~nm}$. Even though the two additives had dissimilar AFEF trends during mode 11 , they both demonstrated particle concentration reductions of $2 \%$ to $18 \%$ between $9 \mathrm{~nm}$ and $21 \mathrm{~nm}$. In the $24 \mathrm{~nm}$ to $37 \mathrm{~nm}$ range, the use of low cetane B10A and low cetane B20A over the base fuel appears to have resulted in $22 \%$ to $37 \%$ reductions and $3 \%$ to $24 \%$ increases in PM concentration, respectively, during mode 11 . 


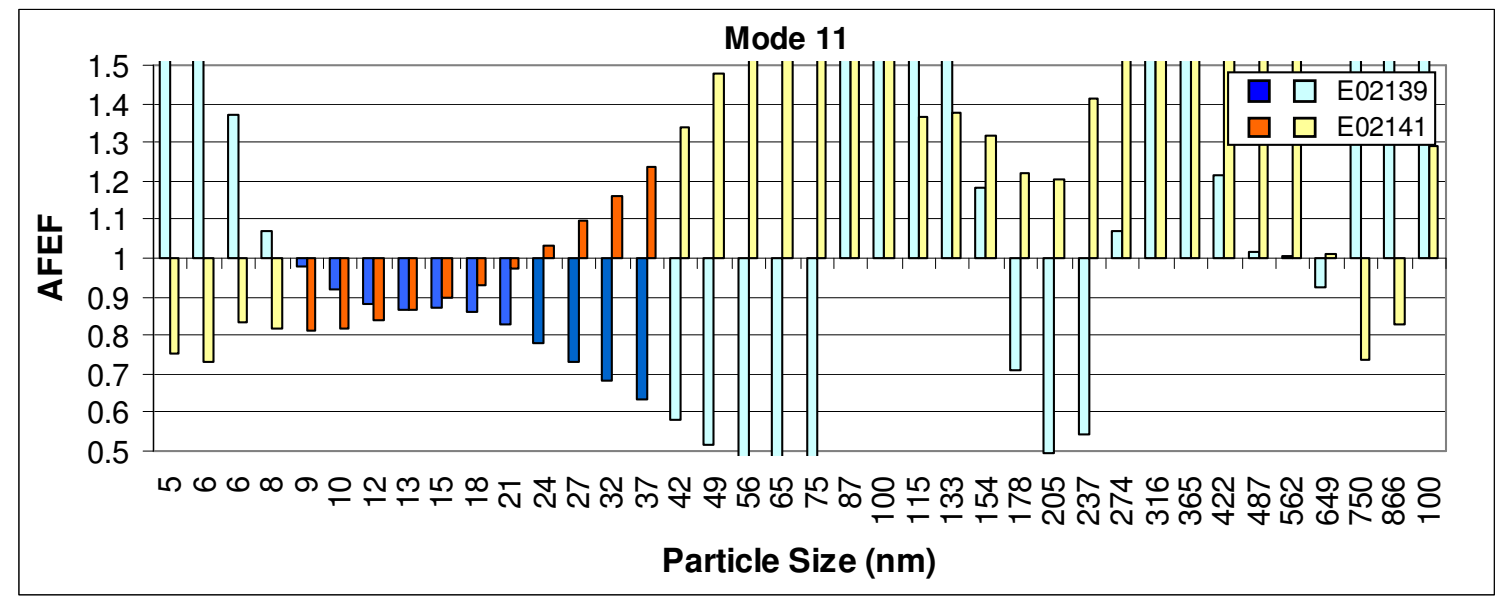

Figure 91 Apparent Fuel Effects of Biodiesel Addition to Low Cetane Diesel for Mode 11

The two AFEF trends of mode 11 may have been similar if repeat testing had been performed. The AFEF curves of modes 2 and 10 were of the same general shape; which is probably because the engine operating conditions of modes 2 and 10 were along the same boundary, as depicted by Figure 71. For the same reason, modes 7 and 11 had a common AFEF trend for the low cetane B20 fuel. As a general rule, the effects that fuel additives or any other variables have on particle concentrations are specific to certain engine operating conditions. For instance, a fuel additive cannot decrease $500 \mathrm{~nm}$ particle concentrations for engine operation that already emits practically no $500 \mathrm{~nm}$ particles.

The steady state AFEF results were of unknown certainty, because repeat tests were not executed. As a means of validation, however, the AFEF results were related to the low cetane, low cetane B10A, and low cetane B20A results of the Base Fuels vs. Additized Fuels section. The FTPs and SETs of these three fuels were performed on the same day with the same engine. For the average engine operating conditions of the repeat FTPs, the use of low cetane B10A provided significant reductions of $3 \%$ to $14 \%$ 
in the nuclei mode particles and $4 \%$ to $16 \%$ in the accumulation mode particles. The use of low cetane B20A in the FTPs resulted in significant reductions of $6 \%$ to $23 \%$ in the nuclei mode particles and $5 \%$ to $36 \%$ in the accumulation mode particles. The average nuclei mode particle distribution of the FTP tests was closest to that of mode 7, and the average accumulation mode particle distribution was closest to that of mode 5 . Therefore, the results of modes 7 and 5 were simultaneously compared to the FTP results. During the steady state operation of mode 7, the use of low cetane B10A was attributed to nuclei mode particle reductions of between $2 \%$ and $10 \%$ (3\% to $14 \%$ for transient tests), and the use of low cetane B20A caused nuclei mode reductions of $2 \%$ to $14 \%$ (6 $\%$ to $23 \%$ for transient tests). For the steady state conditions of mode 5, low cetane B10A was responsible for decreases in accumulation mode particles of between $6 \%$ and $13 \%$ ( $4 \%$ to $16 \%$ for transient tests), and the use of low cetane B20A resulted in accumulation mode particle reductions of $14 \%$ to $33 \%$ (5\% to $36 \%$ for transient tests).

\subsubsection{Dilution Ratio Effects}

Effects of dilution ratio on particle concentrations were studied by using the Guttman fuel, the rebuilt DDC S60, and the SET. There were three SETs performed, one for each primary tunnel flow setting. Test E02208_01 was associated with the "high" primary dilution caused by a main tunnel flow rate of $\sim 3400 \mathrm{scfm}$. With a tunnel flow rate of $2400 \mathrm{scfm}$, E02206_02 was characterized by what was considered to be the standard primary dilution; and E02208_02 was run with the "low" primary dilution that was caused by a tunnel flow rate of $\sim 1400 \mathrm{scfm}$. More detail on the method used to vary the primary dilution ratio can be found on page 69 of the Experimental Setup and Procedures section. A term similar to AFEF, the apparent dilution effect factor (ADEF), 
was essentially the same as the AFEF, and it was used in the following figures and discussion. It was implemented to allow estimations of differences in particle concentrations by comparing the high and low dilution results to the standard dilution results. Since no repeat tests were performed and the same test day, test procedure, fuel, and engine were used as in the steady state humidity tests, the size ranges of acceptable run-to-run variations (from the humidity results) were applied to these results. Only concentration differences of particles within the acceptable ranges were considered for significance. Figures 92 through 97 represent the dilution dependant results of modes 2 , $5,6,7,10$, and 11 . The ADEF values of the high dilution setting are represented by light and dark blue columns, where the dark blue columns indicate differences that were potentially significant. The red and light orange columns represent the ADEF values of the low dilution setting, where red columns suggest significance. Note that the y-axis scales of Figures 95 and 97 are different from those of Figures 92, 93, 94, and 96. Overall dilution ratios of the individual modes are provided by Table 15 below and related to the particle concentration and distribution results of the next six figures. As explained previously, the volumetric particle concentrations were converted to brakespecific concentrations so that the effects of dilution ratio could be studied by visual inspection of the figures below.

Table 15 Average Overall Dilution Ratios of Modes 2, 5, 6, 7, 10, and 11

\begin{tabular}{|c|c|c|c|c|c|c|}
\hline Test Number & Mode 2 & Mode 5 & Mode 6 & Mode 7 & Mode 10 & Mode 11 \\
\hline E02206_02 & 95.6 & 149 & 115 & 186 & 64.0 & 131 \\
\hline E02208_01 & 139 & 216 & 167 & 265 & 94.8 & 190 \\
\hline E02208_02 & 53.0 & 82.7 & 62.8 & 104 & 34.5 & 72.4 \\
\hline
\end{tabular}


The dilution ratio values of Table 15 are discussed throughout this section. Equation 16 from the Continuous FTP Data section was used to calculate the overall dilution ratios.

During the test with the standard dilution setting, the overall dilution ratio of mode 2 was 95.6. The high and low dilution settings resulted in total dilution ratios of 139 and 53.0, respectively, during mode 2. The ADEF values of mode 2 are provided below in Figure 92. The high dilution setting resulted in increases of $5 \%$ to $39 \%$ in nuclei mode particle concentrations, differences of less than $3 \%$ between $50 \mathrm{~nm}$ and 100 $\mathrm{nm}$, and increases of $2 \%$ to $22 \%$ between $100 \mathrm{~nm}$ and $650 \mathrm{~nm}$. The low dilution setting demonstrated nuclei mode concentration reductions of $28 \%$ to $66 \%$ and accumulation mode reductions of $28 \%$ to $7 \%$. The red columns in Figure 92 that are between the 487 $\mathrm{nm}$ and $866 \mathrm{~nm}$ particle sizes denote concentration increases of $18 \%$ to $45 \%$, which may have been due to noise.

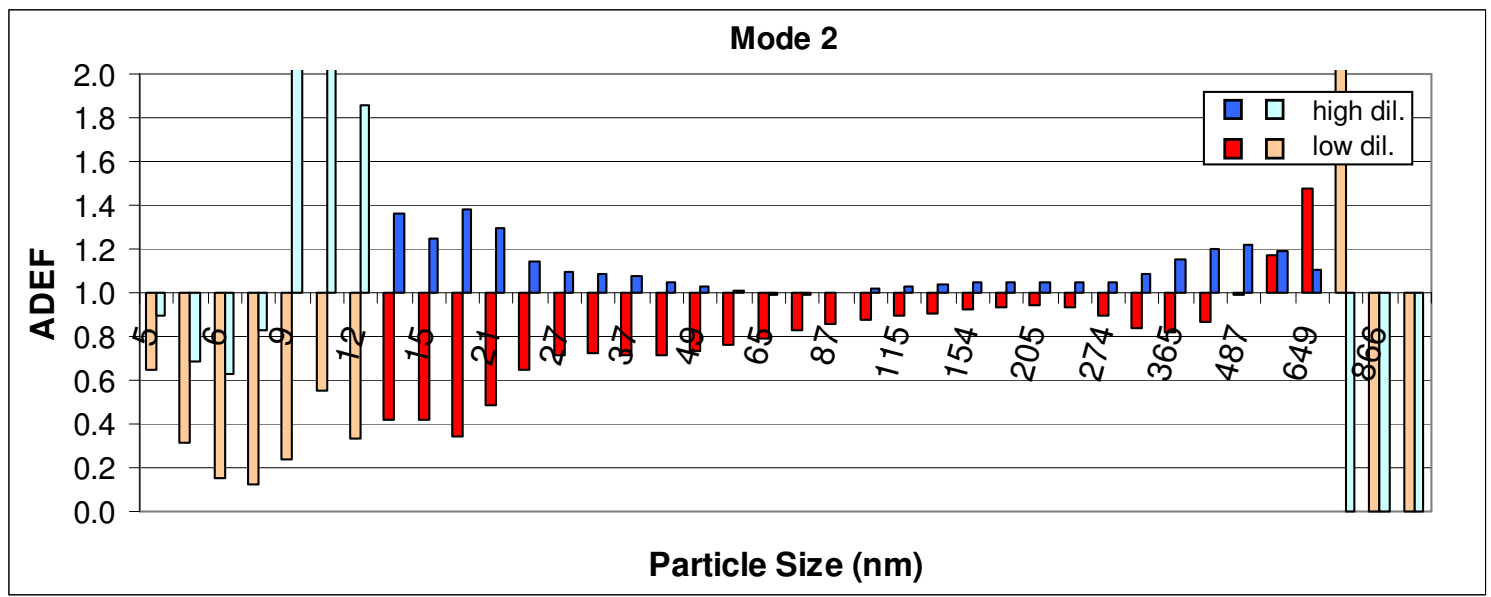

Figure 92 Apparent Dilution Ratio Effects on Particle Concentrations of Mode 2

The low, normal (standard), and high dilution settings resulted in overall dilution ratios of $82.7,149$, and 216 , respectively, in mode 5 . The particle distributions of mode 5 shared a common range of $5 \mathrm{~nm}$ to $250 \mathrm{~nm}$. The resulting ADEF values are provided 
below by Figure 93. In the humidity results of mode 5, the average run-to-run COV values were less than $35 \%$ for the particles smaller than $27 \mathrm{~nm}$. This means that the differences between concentrations of the low and normal dilution settings, which were reductions as great as $70 \%$, can be considered significant between $6 \mathrm{~nm}$ and $20 \mathrm{~nm}$. The acceptable range may not have been applicable to the dilution results of mode 5, because the particle concentrations for sizes greater than $250 \mathrm{~nm}$ were below the detectable limit of the DMS. Therefore, it is suggested that the ADEF values of the high dilution setting were significant only between $27 \mathrm{~nm}$ and $250 \mathrm{~nm}$, and the ADEF values of the low dilution setting were only significant for the ranges of $6 \mathrm{~nm}$ to $20 \mathrm{~nm}$ and $27 \mathrm{~nm}$ to 250 $\mathrm{nm}$. For the $27 \mathrm{~nm}$ to $250 \mathrm{~nm}$ particles of mode 5, conclusions were made that the high dilution setting caused concentration reductions of up to $10 \%$, and the low dilution setting exhibited reductions of up to $15 \%$.

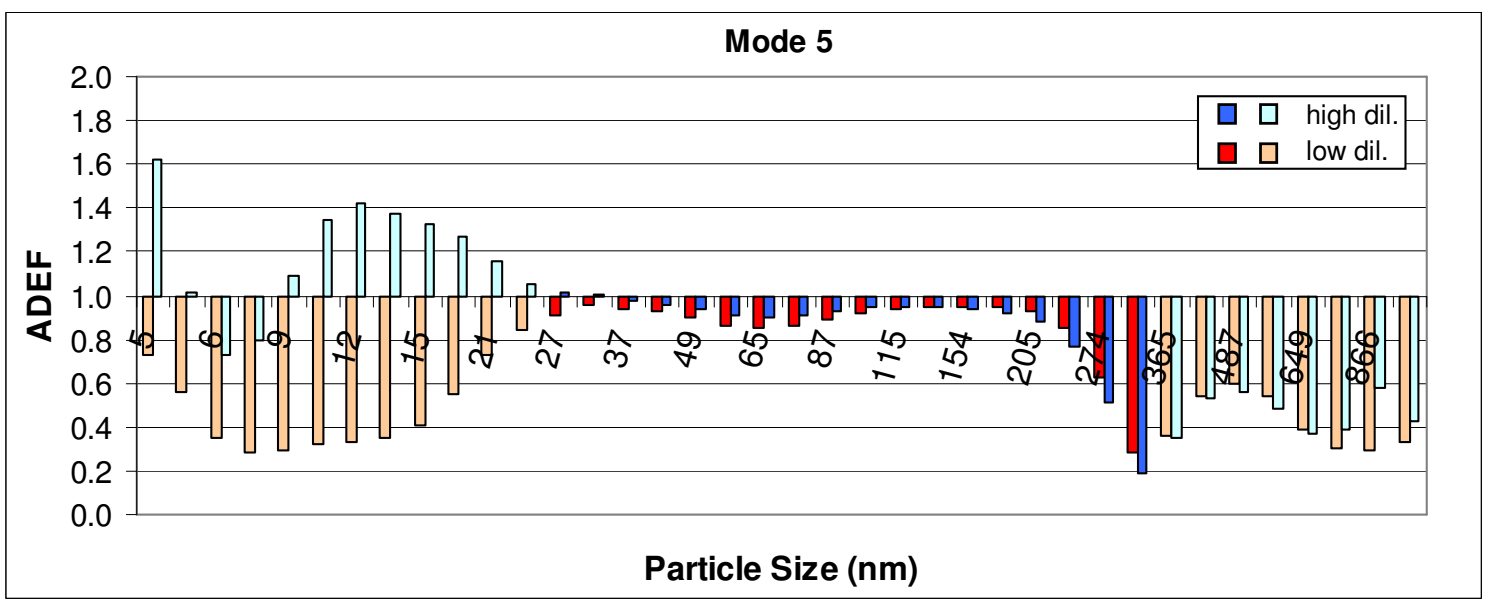

Figure 93 Apparent Dilution Ratio Effects on Particle Concentrations of Mode 5

The dilution results of mode 6 can be found in Figure 94. Mode 6 had low, normal, and high overall dilution ratios of $62.8,115$, and 167 , respectively. The test with the high dilution setting displayed nuclei mode particle concentrations that were $2 \%$ to $47 \%$ greater than those of the normal dilution test. Low dilution was associated with 
nuclei mode reductions of $18 \%$ to $59 \%$. The particles of $40 \mathrm{~nm}$ to $200 \mathrm{~nm}$ were not significantly affected (differences were less than $5 \%$ ) by use of the high dilution setting, and they were reduced by $5 \%$ to $17 \%$ when the low dilution setting was employed. Particles of mode 6 that were between $200 \mathrm{~nm}$ and $500 \mathrm{~nm}$ appear to be affected by dilution ratio, but the results were randomly distorted by measurement errors of unknown causes.

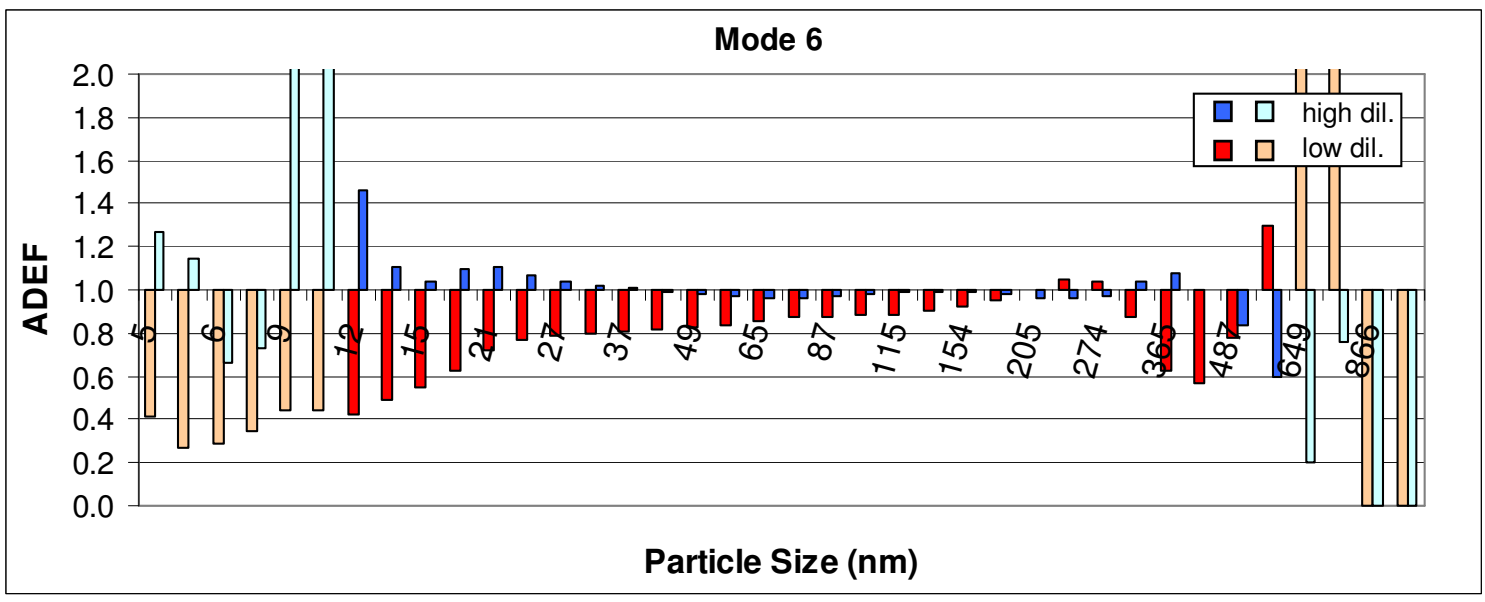

Figure 94 Apparent Dilution Ratio Effects on Particle Concentrations of Mode 6

The maximum total dilution ratios of the low $\left(R_{o}=104\right)$, normal $\left(R_{o}=186\right)$, and high $\left(R_{o}=265\right)$ dilution tests occurred during mode 7 . Figure 95 shows the ADEF values for mode 7 of the steady state dilution test. The comparisons indicated that raising the dilution ratio caused concentration increases of $5 \%$ to more than $100 \%$ (close inspection of data indicated a peak increase of $175 \%$ ) in the $8 \mathrm{~nm}$ to $37 \mathrm{~nm}$ size range, and lowering the dilution ration led to particle concentration reductions of $20 \%$ to $84 \%$ in the $8 \mathrm{~nm}$ to $65 \mathrm{~nm}$ size range. The high dilution setting also caused reductions of between $31 \%$ and $100 \%$ in some particle concentrations of the accumulation mode. 


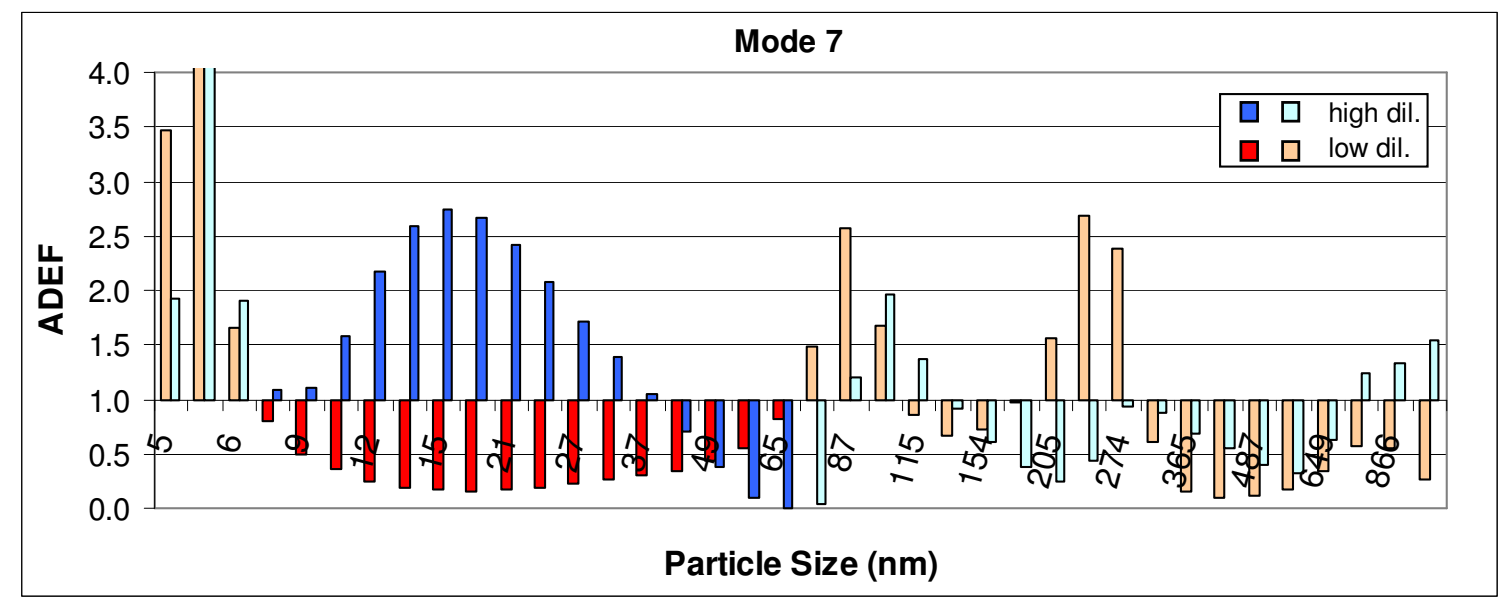

Figure 95 Apparent Dilution Ratio Effects on Particle Concentrations of Mode 7

Mode 10 particle concentration comparisons for the three dilution settings can be found in Figure 96. The low dilution setting yielded an average overall dilution ratio of 34.5, and the standard and high settings provided ratios of 64.0 and 94.8, respectively. The particle concentrations of the high dilution ratio appeared to be $6 \%$ to $16 \%$ higher in the $15 \mathrm{~nm}$ to $27 \mathrm{~nm}$ particles, not significantly affected in the $30 \mathrm{~nm}$ to $150 \mathrm{~nm}$ particles, and $5 \%$ to $27 \%$ lower in the $154 \mathrm{~nm}$ to $487 \mathrm{~nm}$ particles when compared to the concentrations of the standard dilution ratio. Use of the low dilution ratio setting during mode 10 resulted in particle concentration reductions of $57 \%$ to $15 \%$ between $15 \mathrm{~nm}$ and $50 \mathrm{~nm}, 7 \%$ to $20 \%$ between $100 \mathrm{~nm}$ and $205 \mathrm{~nm}$, and $15 \%$ to $34 \%$ between $315 \mathrm{~nm}$ and $420 \mathrm{~nm}$. The low dilution ratio may have also caused concentration gains in the particles between $65 \mathrm{~nm}$ and $87 \mathrm{~nm}$. 


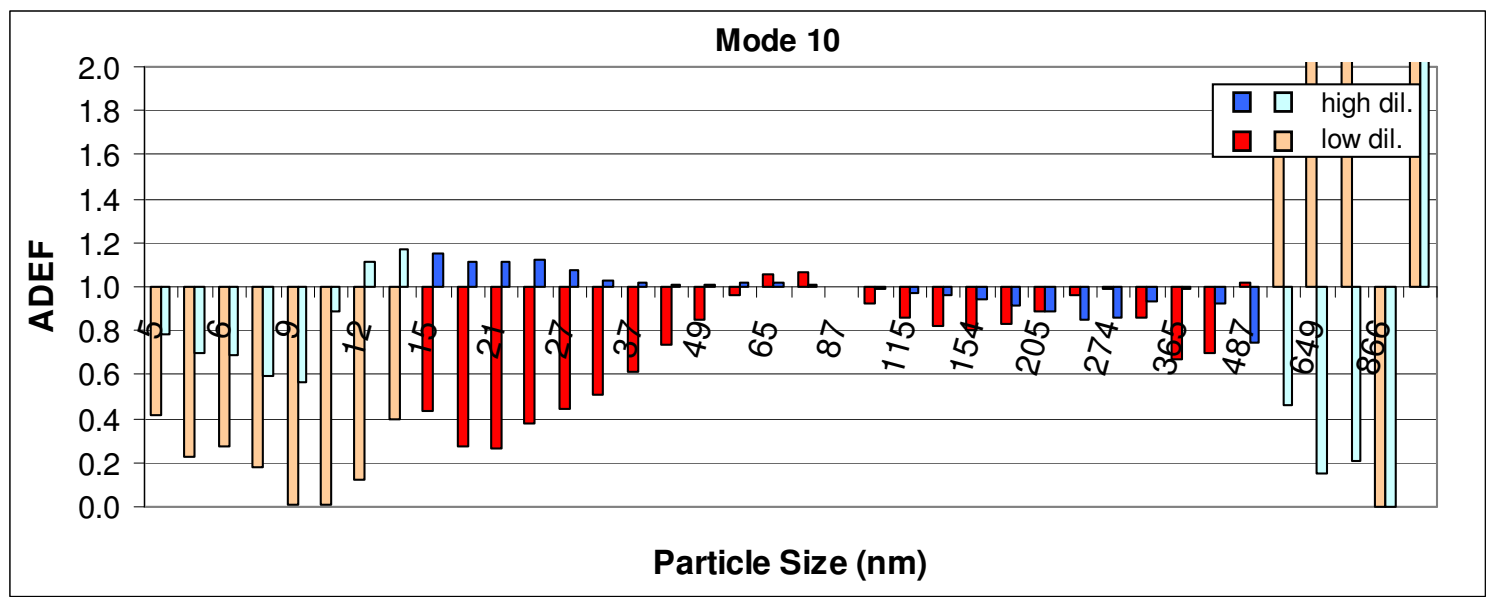

Figure 96 Apparent Dilution Ratio Effects on Particle Concentrations of Mode 10

The high, standard, and low overall dilution ratios of mode 11 were $72.4,131$, and 190, respectively; and the apparent particle concentration differences can be found in Figure 97. Between $9 \mathrm{~nm}$ and $65 \mathrm{~nm}$, none of the average run-to-run COV values of the humidity tests were greater than $30 \%$. Therefore, the acceptable range was once again ignored, and the concentration differences of the $9 \mathrm{~nm}$ to $65 \mathrm{~nm}$ particles (of the dilutionspecific tests) that were greater than $30 \%$ were considered to be significant. The ADEF values of Figure 97 indicate that increasing the primary tunnel flow from $~ 2400 \mathrm{scfm}$ to 3400 scfm caused particle concentrations of mode 11 to increase by anywhere from 10 $\%$ to $328 \%$ in the $9 \mathrm{~nm}$ to $65 \mathrm{~nm}$ size range. Mode 11 was the only example where the low dilution test emitted more particles (35\% to $150 \%$ ) per unit work than the standard dilution test between $9 \mathrm{~nm}$ and $15 \mathrm{~nm}$. The particles between $18 \mathrm{~nm}$ and $65 \mathrm{~nm}$ were subject to concentration reductions of $25 \%$ to $90 \%$ when the tunnel flow rate was lowered from $\sim 2400 \mathrm{scfm}$ to $\sim 1400 \mathrm{scfm}$. 


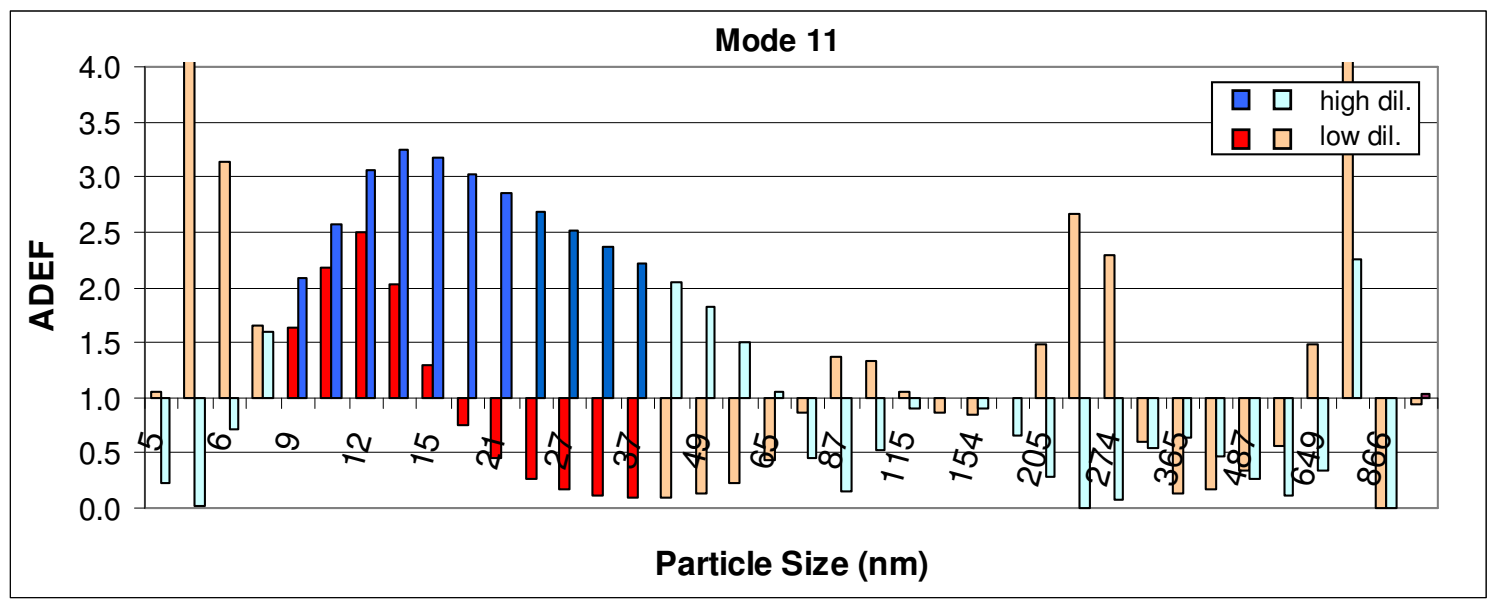

Figure 97 Apparent Dilution Ratio Effects on Particle Concentrations of Mode 11

Changes in the dilution setting caused variations in residence time of up to 1.88 seconds. As discussed on page 15 , research has shown that residence time is a prominent factor in nanoparticle formation. The higher dilution settings provided lower tunnel temperatures, which are required for the condensation of gaseous compounds into volatile particles. This is one of the mechanisms with which the higher dilution ratios caused increases in nuclei mode particle concentrations. It was found in the literature that only nuclei mode particles are affected by dilution ratio [28]. This research provided evidence that, for the selected fuel and engine, dilution ratio always affects the nuclei mode; and under specific operating conditions, it affects the accumulation mode. The nuclei mode was reduced in all circumstances of the steady state dilution-specific testing in which the dilution ratio was lowered. The increase of $9 \mathrm{~nm}$ to $15 \mathrm{~nm}$ particle concentrations (shown by red columns in Figure 97) during mode 11 appeared to be an exception; but upon inspection of Figure A-76 in the Appendix, it was determined that the entire nuclei mode was also reduced for mode 11 when the dilution ratio was decreased. The peak of the nuclei mode during mode 11 occurred at $25 \mathrm{~nm}$ with the normal dilution setting; and it occurred at $15 \mathrm{~nm}$ with the low dilution setting. This means that the nuclei mode was 
reduced, but it was also shifted. The increase in concentrations between $9 \mathrm{~nm}$ and $15 \mathrm{~nm}$ were due merely to variation in the span of the nuclei mode distribution. When the dilution ratio was decreased, the brake-specific concentrations of accumulation mode particles smaller than $205 \mathrm{~nm}$ were either reduced slightly (5\% to $20 \%$ ) or were not significantly affected $(<5 \%)$. Above $205 \mathrm{~nm}$, the concentrations seemed to be randomly affected by dilution ratio, which could have been due to measurement error. Once more, no repeat tests were executed, so the results were suggestive instead of conclusive. Applicability of the acceptable ranges was voided in a few cases, because changes in overall dilution ratio caused shifts in the modal distributions of particles. Continuous concentration contour plots (volumetric basis) of the high, standard, and low dilution tests are provided on pages 224, 225, and 226, respectively, of the Appendix. Brake-specific concentration plots for the high and low dilution settings of modes 2, 5, 6, 7, 10, and 11 are provided on pages 221 through 223 of the Appendix. The shifts in modal distributions, such as the one in the nuclei distribution of mode 11, can be visually detected from the contour plots (Figures A-74, A-75, and A-76). It was also apparent from the contour plots that the particle distributions of modes $2,5,6,7,10$, and 11 remained transient. A longer period of operation for each mode might have resulted in greater consistency of the spectral particle concentrations. The results above and the conclusions of this paragraph are specific to the selected fuel, engine, and modes of the SET. 


\subsubsection{Secondary Dilution Ratio Validation}

The secondary dilution ratio $\left(R_{s}\right)$, which was needed in equation 16 to calculate the overall dilution ratios, was originally set to 25 . Validation of the actual secondary dilution ratio was done by measuring the oxides of nitrogen before and after the secondary dilution system. The secondary dilution ratios illustrated below by Figure 98 were calculated by dividing the gaseous concentration values $\left(\mathrm{NO}_{\mathrm{x}} \mathrm{ppm}\right)$ of the analyzer that sampled from the primary tunnel by the values (Remote $\mathrm{NO}_{\mathrm{x}} \mathrm{ppm}$ ) of the analyzer that took measurements from the secondary dilution mixture. The dilution ratios of Figure 98 are those of the E02206_02 SET. Excluding the ones that are shown for mode 1 (between 0 seconds and 150 seconds), the calculated secondary dilution ratios of E02206_02 were between 23.5 and 24.6.

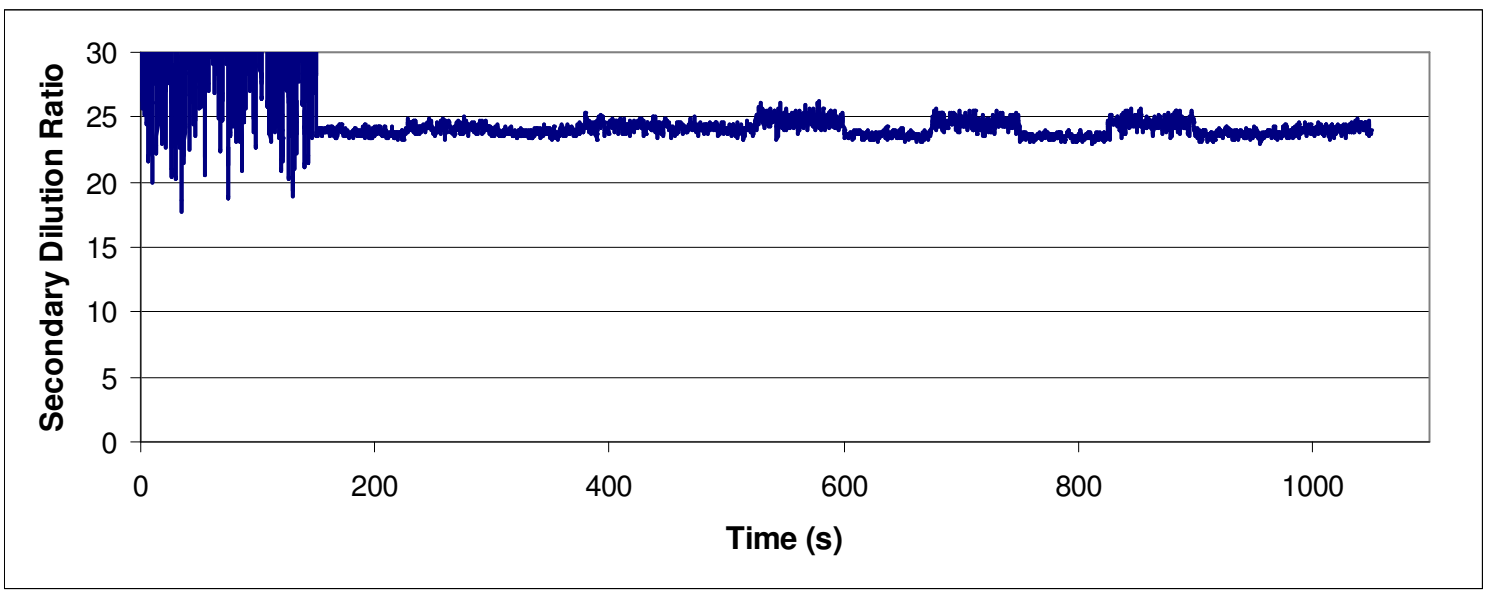

Figure 98 Calculated Secondary Dilution Ratios of Steady State Test E02206_02

Figure 99 indicates the $\mathrm{NO}_{\mathrm{x}}$ concentrations of the primary and remote $\mathrm{NO}_{\mathrm{x}}$ analyzers during E02206_02. The concentrations measured by the remote $\mathrm{NO}_{\mathrm{x}}$ analyzer were below the detection limit during mode 1 . This is why the secondary dilution ratios of mode 1, which are shown in Figure 98, appear to be scattered. Some of the other variations in the calculated secondary dilution ratios were attributed to measurement 
errors as well; the remote $\mathrm{NO}_{\mathrm{x}}$ concentrations were occasionally less than $6 \mathrm{ppm}$ and the remote $\mathrm{NO}_{\mathrm{x}}$ analyzer was mistakenly calibrated to $50 \mathrm{ppm}$.

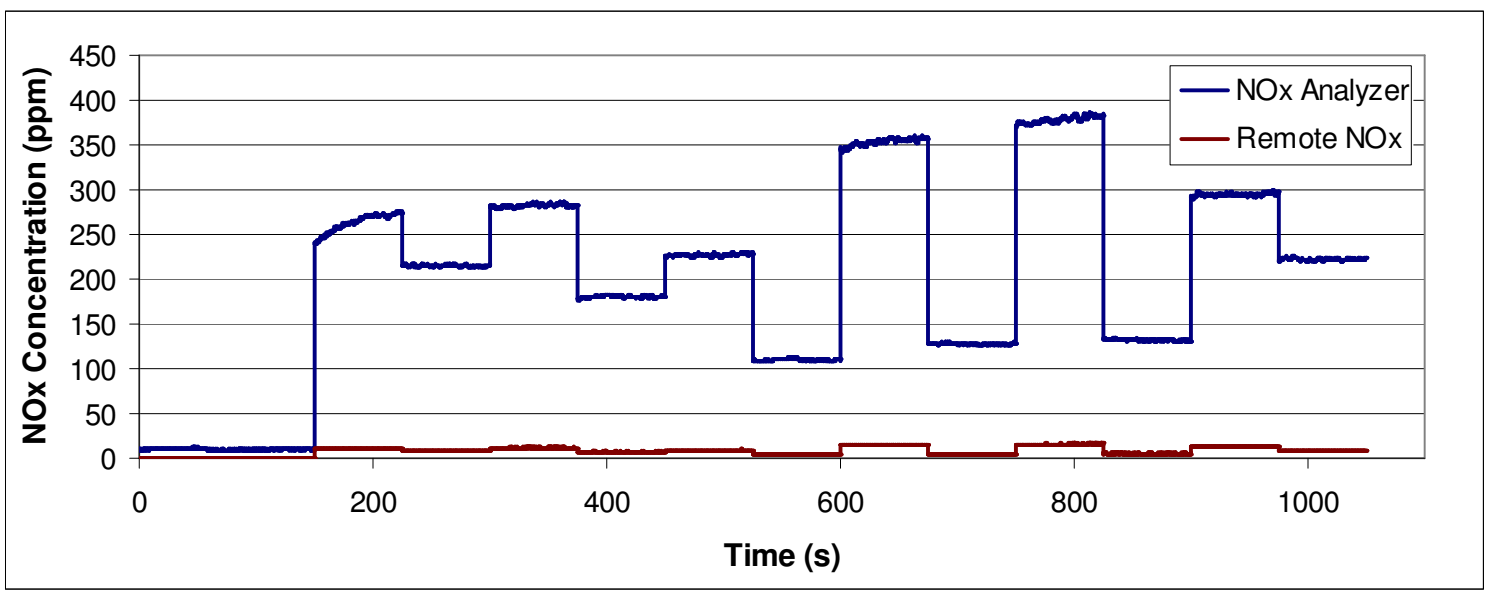

Figure $99 \mathrm{NO}_{\mathrm{x}}$ and Remote $\mathrm{NO}_{\mathrm{x}}$ Concentrations of Steady State Test E02206_02

With $\mathrm{NO}_{\mathrm{x}}$ measurements of mode 1 ignored, the average secondary dilution ratios of nine SETs were calculated. These average secondary dilution ratios can be found in Figure 100. The light blue columns represent the dilution ratio values that were calculated by use of $\mathrm{NO}_{\mathrm{x}}$ measurements, and the light purple columns show those that were calculated with the $\mathrm{NO}_{\mathrm{x}} 2$ measurements. The standard deviation bars, or error bars, represent the standard deviations between the secondary dilution ratio values of each test. The greatest average secondary dilution ratio that was calculated (24.5) was for E02204_01, and the lowest (23.6) was for E02205_01. The average secondary dilution ratio for the nine tests was calculated to be 24.0. 


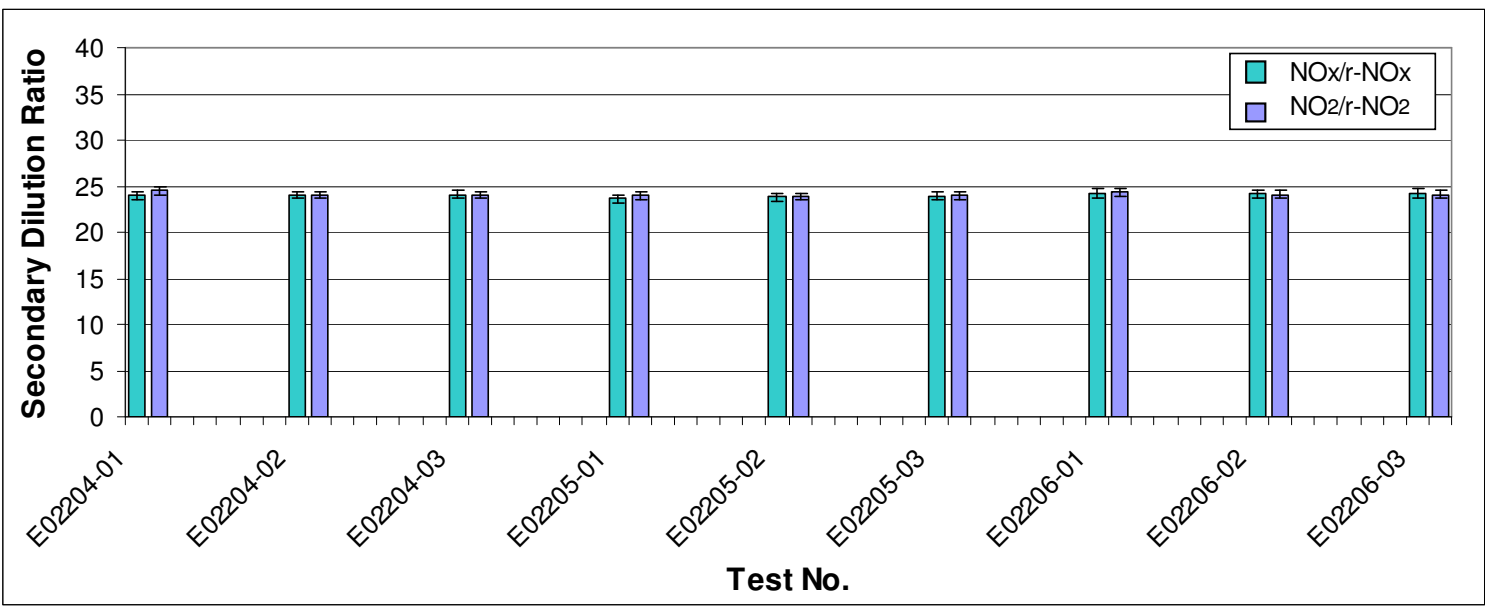

Figure 100 Average Secondary Dilution Ratios of Humidity-Specific SETs

Consistency of the secondary dilution ratio was essential for measurement precision, and the secondary dilution system provided satisfactory performance (the calculated precision of the secondary dilution ratio was $24.0+-2.5 \%$ ). The average secondary dilution ratio value of 24.0 was used in all of the overall dilution ratio calculations. 


\section{CONCLUSIONS}

The research was completed to provide a better understanding of the variability in DPM size measurements from HDDEs and to determine how measurements are affected by certain parameters. The independent variables were: fuel type, sample line temperature, relative humidity within the dilution tunnel, and overall dilution ratio. To put the testing circumstances in perspective $(63.6<\mathrm{DR}<445)$, the dilution conditions of this study were similar to what a motorcycle rider would experience if he/she maintained several feet of distance behind a moving tractor-trailer. For PM size and concentration measurements to ever be mandatory for HDDE testing, standardization of the sampling technique must be declared. Many scientific establishments, such as HEI, are currently investigating human health issues associated with diesel particulates, and spectral particle concentration measurements may eventually help to determine the long-term risks of certain working environments.

\subsection{EXTREMES OF RUN-TO-RUN VARIATION (objective 1)}

The run-to-run variations, or COV values, were used to determine which differences were significant. The run-to-run variations of the E02138 test sequence were as low as $0.08 \%$, indicating that it would have been possible to detect significant particle concentration differences of $\sim 0.16 \%$ if a test sequence of similar variance were compared. The lowest run-to-run variations of the least repeatable test sequence, E01921, were $1.08 \%$. This means that for all of the FTP testing, minimum significant differences of less than $2.2 \%$ could have been determined for certain particle sizes. The COV trend lines, which were dependant on the nature of particle losses for each engine model and fuel type, differed in magnitude for each particle size. The smallest significant 
difference that was found during this research was a $1.51 \%$ increase in $65 \mathrm{~nm}$ particles due to the addition of the "trt. 1" additive (test sequence E01947) to the low cetane fuel (test sequence E01943). The largest significant difference that was found was $125 \%$ between the $32 \mathrm{~nm}$ particles of test fuels FEM trt. 3 (E02195 test sequence) and FEM trt. 6 (E02198 test sequence). When either of the 1992 Detroit Diesel DDC S60s were used, it was generally possible to detect differences of $4 \%$ or less between $50 \mathrm{~nm}$ and $150 \mathrm{~nm}$ and $10 \%$ or less between $20 \mathrm{~nm}$ and $200 \mathrm{~nm}$. In most cases with the 2004 Cummins ISM 370 , it was possible to detect differences of $6 \%$ or less between $50 \mathrm{~nm}$ and $150 \mathrm{~nm}$ and $16 \%$ or less between $20 \mathrm{~nm}$ and $200 \mathrm{~nm}$. Even if standardization of PM size and concentration measurements is implemented, the minimum detectable variations will be inconsistent between fuel and engine combinations.

\subsection{HUMIDITY EFFECTS (objective 2)}

The humidity results were specific to the rebuilt DDC S60 engine and Guttman fuel combination, and it was noted that the run-to-run variations were not affected by humidity. Significant differences in particle concentrations due to changes in relative humidity within the dilution tunnel were only noticed for the low load $(<50 \%)$ engine operation of modes 5, 7, and 11. During mode 5, the low $(\sim 37 \%)$ and high $(\sim 88 \%)$ humidity settings both showed reductions of between $10 \%$ and $67 \%$ in the $205 \mathrm{~nm}$ to $487 \mathrm{~nm}$ size range, when compared to the standard ( 60\%) humidity setting. Compared to the standard humidity setting of mode 7 , there were concentration reductions of $8 \%$ to $24 \%$ in the $7 \mathrm{~nm}$ to $10 \mathrm{~nm}$ particles when the low humidity setting was applied. For mode 11 , the high humidity setting resulted in $9 \%$ to $88 \%$ increases between $24 \mathrm{~nm}$ and $100 \mathrm{~nm}$, and the low humidity setting caused reductions of $22 \%$ to $83 \%$ in the $5 \mathrm{~nm}$ to 7 
$\mathrm{nm}$ and $154 \mathrm{~nm}$ to $275 \mathrm{~nm}$ particles. Since the effects of decreasing the relative humidity did not mirror those of increasing the relative humidity, it is suggested that the mechanisms by which the different humidity settings altered the particle concentrations were not the same. Perhaps the injection of steam (to raise the humidity levels) caused changes in average tunnel temperature (which were typically between $0.2{ }^{\circ} \mathrm{C}$ and $3.4{ }^{\circ} \mathrm{C}$ ) that were more influential on particle formation than the humidity variations.

\subsection{DILUTION RATIO EFFECTS (objective 3)}

The dilution results were also specific to the rebuilt DDC S60 engine and Guttman fuel combination. The higher overall dilution ratios provided greater cooling, which led to greater amounts of nanoparticles due to increased nucleation. Consequently, the higher dilution settings caused increases in nanoparticle concentrations in every case (SET modes 2, 5, 6, 7, 10, and 11). The accumulation mode particle concentrations increased in some cases (SET modes 2, 5, 6, and 10) as the overall dilution ratio was elevated, but not by as much as the nuclei mode particles.

\subsection{DAY-TO-DAY VARIATION (objective 4)}

With each fuel and engine combination that was used for day-to-day variation testing, the run-to-run COV trends were always different from one day to the next. It was assumed that barometric pressure changes contributed to the day-to-day variations, since barometric pressure was the only parameter that was not controlled. It was suspected that a change in barometric pressure from an average value of 14.155 psi during the E01927 test sequence (day 1) to an average value of 14.330 psi during the E01928 test sequence (day 2) caused day-to-day concentration variations of between $3 \%$ and $10 \%$ in the 32 
$\mathrm{nm}$ to $316 \mathrm{~nm}$ particles. It was also likely that hourly changes in barometric pressure were responsible for portions of the run-to-run variations.

\subsection{BASE FUELS VS. ADDITIZED FUELS (objective 5)}

Each additive that was studied caused significant differences in particle concentrations of higher than $10 \%$. In all cases, an increase in the amount of the additive led to an increase in the resulting particle concentration differences. The greatest difference due to the use of an additive was a $130 \%$ increase in $32 \mathrm{~nm}$ particles. The use of cetane improving additives generally resulted in concentration reductions for all of the measured particle sizes that were affected. The blending of biodiesels with petroleumbased fuels has been known to reduce PM mass-based emissions; and in this research (for the rebuilt 1992 DDC S60), it always reduced the particle concentrations of most sizes and did not affect those of the other sizes.

\subsection{COMPARISON OF TEOM AND DMS DATA (objective 6)}

By use of the technique described in the Comparison of TEOM and DMS Data section that was developed for the conversion of DMS spectral density data into continuous PM mass data, it is possible to use the DMS as a measurement tool for TPM and the mass flow of PM. The use of the DMS instead of the TEOM to measure PM mass flow eliminates the need for correction of water absorption onto the TEOM filter. Possibly due to averaging within the TEOM, the DMS detected impulses in PM mass flow that the TEOM did not. The effective density technique also provides estimations for the contributions of each bin (particle size range) to TPM. When the rebuilt DDC S60 was used, the DMS TPM consistently agreed with the gravimetric filter TPM to within $6.25 \%$. The DMS TPM of the non-rebuilt DDC S60 was always less than $4 \%$ 
different than the gravimetric filter TPM. With the ISM 370, however, the DMS TPM was consistently $18.2 \%$ to $26.4 \%$ lower than the gravimetric filter TPM. This means that the effective density constants that were used are not applicable to newer engine technologies, such as EGR on the ISM 370. If the effective density constants were determined for a variety of engine types, the accuracy of the DMS PM mass measurement technique might approach or surpass the accuracy of the TEOM.

\subsection{SAMPLE LINE TEMPERATURE EFFECTS (objective 7)}

The run-to-run variation was higher for test sequence E01967, which had an unheated $\left(32{ }^{\circ} \mathrm{C}\right.$ average temperature) sample line, than for test sequence E01968, which had a heated $\left(47+-1.0^{\circ} \mathrm{C}\right.$ constant temperature $)$ sample line. This was probably because deviations in the sample line temperature resulted in variations of mass flow through the ejector diluter, which caused variations in the secondary dilution ratio. The only particle concentration that seemed to be significantly affected by sample line temperature was that of the $5 \mathrm{~nm}$ particles. The $5 \mathrm{~nm}$ particles were increased in concentration by $15 \%$ when the sample line heater was turned off. In the existing literature, it is commonly reported that the temperature within the dilution environment is related to the formation of nanoparticles. The conclusion of this sample line temperature study was probably not the same because particles spent no more than 0.088 seconds in the sample line (between the primary tunnel and the secondary dilution system), and the final sample temperature at the intake of the DMS was $\sim 25^{\circ} \mathrm{C}$ regardless of the sample line temperature.

\subsection{CONTINUOUS FTP DATA (objective 8)}

It was observed that each engine operating event during an FTP was characterized by a unique emission of particulate matter. At high engine speed and low load, there was 
typically a wide and flat accumulation mode with no distinct peak concentration. Under the condition of low engine speed and a small negative load, which occurs during light motoring of the engine, there was a bi-modal distribution; where the nuclei mode had a peak concentration at around $12 \mathrm{~nm}$ that was generally more than an order of magnitude higher than the greatest concentration of the accumulation mode. An engine operating condition of low speed and high load resulted in an accumulation mode distribution with very high particle concentrations in the $100 \mathrm{~nm}$ to $130 \mathrm{~nm}$ size range. Such an event was typical when there was a sudden increase in load that led to turbo lag and a low pitched "grumbling" noise of the engine, and it usually corresponded to the greatest instantaneous mass flow rate of PM during an FTP. The equivalence ratio is typically high during turbo lag, and it is known that high equivalence ratios lead to high levels of PM. Engine idle was characterized by very low concentrations of nanoparticles and the lowest mass flow rate of PM. Engine "flutter," or small repetitive fluctuations in engine speed during idle, was associated with random shifts in the range of the nanoparticle distribution. It was also noticed that the emission of nanoparticles during idle lessened as the lube oil temperature increased. High engine speed and high load operation, such as during a fullthrottle hill climb or acceleration, resulted in the widest distribution of accumulation mode particles with a fairly high peak concentration at about $40 \mathrm{~nm}$. The greatest concentrations of nanoparticles occurred when there was a sudden shift from high power output to immense energy absorption of the engine. Directly after the shift in power, nanoparticle concentrations were very high, but they decreased rapidly during the high power engine motoring as the exhaust temperature declined. 


\section{RECOMMENDATIONS}

This research was used to answer basic questions about how certain laboratory variables can affect particle spectrum measurements of diesel particulate matter. There are many extensions of this study that can be explored in order to benefit the scientific community. The following recommendations indicate topics of interest and provide information on possible ways to improve upon the aerosol sampling technique that was used in this study.

- If the relationship between a controllable laboratory parameter and size-specific PM concentrations is to be studied, use an engine that is known to provide optimal repeatability of the gaseous and TPM emissions.

- Increase the length of time of each steady state mode so that the engine oil temperature and particle concentrations will have enough time to stabilize.

- Execute more than three repeat tests per setting so that a more accurate statistical model can be utilized.

- Determine and tabulate the effective density constants for the available engine configurations.

- Investigate the effects of engine oil temperature and age on PM concentrations.

- To increase measurement precision, use a sampling location that will provide the lowest possible residence time.

- For newer engine technologies, such as EGR and DPFs, determine the dilution ratios that provide an optimal balance for the soot accumulation and underranging of the DMS-500.

- Study the particle concentrations of a constant overall dilution ratio by drawing a constant mass-flow of raw exhaust into the dilution system. 


\section{REFERENCES}

1. Code of Federal Regulations, CFR Title 40 Part 86-89, Office of Federal Register, National Archives and Records Administration, Washington, DC, 2006.

2. "Diesel Particulate Matter,” DieselNet, Ecopoint Inc., Brampton, ON, Canada, 2006. http://www.dieselnet.com/tech/dpm/html

3. "Diesel Exhaust Particle Size," DieselNet, Ecopoint Inc., Brampton, ON, Canada, 2006. http://www.dieselnet.com/tech/dpm_size.html

4. Vuk, C.T., Jones, M.A., and Johnson, J.H., "The Measurement and Analysis of the Physical Character of Diesel Particulate Emissions," Warrendale, PA, SAE Technical Paper No. 760131, 1976.

5. Khalek, I.A., "Characterization of Particle Size Distribution of a Heavy-Duty Diesel Engine During FTP Transient Cycle Using ELPI," Warrendale, PA, SAE Technical Paper No. 2000-01-2001, 2000.

6. "Automobile Emissions: An Overview," Environmental Protection Agency, Washington, DC, 2007. http://www.epa.gov/otaq/consumer/05-autos.pdf

7. "Research on Diesel Exhaust and Other Particulates," Health Effects Institute, Boston, MA, 2003. www.healtheffects.org

8. "Health Effects of Diesel Particulates," DieselNet, Ecopoint Inc., Brampton, ON, Canada, 2007. http://www.dieselnet.com/tech/health_pm.html

9. "Diesel Particulate Matter," Environmental Protection Agency, Washington, DC, 2007. http://www.epa.gov/region1/eco/airtox/diesel.html

10. Liu, Z.G., Thurow, E.M., Lincoln, J.C., Chen, D., Perera, N., and Pingen, G., "Transient Analysis of Engine Nano-Particles Using a Fast-Scanning Differential Mobility Particle Analyzer," Warrendale, PA, SAE Technical Paper No. 2004-01-0971, 2004.

11. Siegmann, K. and Siegmann, H.C., "Fast and Reliable "in situ" Evaluation of Particles and their Surfaces with Special Reference to Diesel Exhaust," Warrendale, PA, SAE Technical Paper No. 2000-01-1995, 2000.

12. Hall, D.E., Stradling, R.J., Zemroch, P.J., Rickeard, D.J., Mann, N., Heinze, P., Martini, G., Hagemann, R., Rantanen, L., and Szendefi, J., "Measurement of the Number and Size Distribution of Particle Emissions from Heavy Duty Engines," Warrendale, PA, SAE Technical Paper No. 2000-01-2000, 2000. 
13. Lee, K.O. and Zhu, J., "Evolution in Size and Morphology of Diesel Particulates along the Exhaust System," Warrendale, PA, SAE Technical Paper No. 2004-01-1981, 2004.

14. "PM Measurement: In-Situ Methods," DieselNet, Ecopoint Inc., Brampton, ON, Canada, 2007. http://www.dieselnet.com/tech/measure_pm_ins.html

15. Burtscher, H., "Characterization of Ultrafine Particle Emissions from Combustion Systems," Warrendale, PA, SAE Technical Paper No. 2000-01-1997, 2000.

16. Kawano, D., Kawai, T., Naito, H., Goto, Y., Odaka, M., and Bachalo, W., "Comparative Measurement of Nano-Particulates in Diesel Engine Exhaust Gas by Laser-Induced Incandescence (LII) and Scanning Mobility Particle Sizer (SMPS)," Warrendale, PA, SAE Technical Paper No. 2004-01-1982, 2004.

17. Goto, Y. and Kawai, T., "Real-Time Measurement of Particle Size Distribution from Diesel Engines Equipped with Continuous Regenerative DPF Under a Transient Driving Condition,” Warrendale, PA, SAE Technical Paper No. 2004-01-1984, 2004.

18. Saito, K., Shinozaki, O., Seto, T., Kim, C., Okuyama, K., Kwon, S., and Lee, K.W., "The Origins of Nanoparticle Modes in the Number Distribution of Diesel Particulate Matter," Warrendale, PA, SAE Technical Paper No. 2002-01-1008, 2002.

19. Tanaka, S. and Shimizu, T., "A Study of Composition and Size Distribution of Particulate Matter from DI Diesel Engine," Warrendale, PA, SAE Technical Paper No. 1999-01-3487, 1999.

20. Pagan, J., "Study of Particle Size Distributions Emitted by a Diesel Engine," Warrendale, PA, SAE Technical Paper No. 1999-01-1141, 1999.

21. Khalek, I.A., Spears, M., and Charmley, W., "Particle Size Distribution from a Heavy-Duty Diesel Engine: Steady-State and Transient Emission Measurement Using Two Dilution Systems and Two Fuels," Warrendale, PA, SAE Technical Paper No. 200301-0285, 2003.

22. Jarrett, R.P. and Clark, N.N., "Evaluation of Methods for Determining Continuous Particulate Matter from Transient Testing of Heavy-Duty Diesel Engines," Warrendale, PA, SAE Technical Paper No. 2001-01-3575, 2001.

23. Reavell, K., Hands, T., and Collings, N., "A Fast Response Particulate Spectrometer for Combustion Aerosols," Warrendale, PA, SAE Technical Paper No. 2002-01-2714, 2002.

24. Virtanen, A., Ristimaki, J., Marjamaki, M., Vaaraslahti, K., Keskinen, J., and Lappi, M., "Effective Density of Diesel Exhaust Particles as a Function of Size," Warrendale, PA, SAE Technical Paper No. 2002-01-0056, 2002. 
25. Kinsey, J.S., Mitchell, W.A., Squier, W.C., Linna, K., King, F.G., Logan, R., Dong, Y., Thompson, G.J., and Clark, N.N., "Evaluation of Methods for the Determination of Diesel-Generated Fine Particulate Matter: Physical Characterization Results," Elsevier, Elsevier Inc., Burlington, MA, 2005. http://www.elsevier.com/locate/jaerosci

26. Collings, N. and Graskow, B.R., "Particles from Internal Combustion Engines What We Need to Know," JSTOR, New York, NY, 2000. http://www.jstor.org

27. Jarrett, R.P., Clark, N.N., Gilbert, M., and Ramamurthy, R., "Evaluation and Correction of Moisture Adsorption and Desorption from a Tapered Element Oscillating Microbalance," Elsevier, Elsevier Inc., Burlington, MA, 2000.

http://www.elsevier.com/locate/powtec

28. Lin, Z.G., Vasys, V.N., Swor, T.A., and Kittelson, D.B., "Significance of Fuel Sulfur Content and Dilution Conditions on Particle Emissions from a Heavily-Used Diesel Engine during Transient Operation," Warrendale, PA, SAE Technical Paper No. 200701-0319, 2007.

29. Tincher, R.R., "Evaluation of Heavy Duty Diesel Engines Regulated Emissions Based on Variation of Fuel Properties by Use of Additives," Masters Thesis, Department of Mechanical and Aerospace Engineering, West Virginia University, Morgantown, WV, 2007.

30. "DMS-500 Fast Particulate Spectrometer User Manual," Cambustion Ltd., Cambridge, UK, 2003. http://www.cambustion.co.uk 


\section{APPENDIX}

\section{A.1 TEST SUMMARY}

Table A-1 Summary of Testing

\begin{tabular}{|c|c|c|c|c|c|c|c|c|}
\hline Engine & Test No. & Test Cycle & Fuel & $\begin{array}{l}\text { Tunnel } \\
\text { Flow } \\
\text { (scfm) }\end{array}$ & $\begin{array}{c}\text { Approx. } \\
\text { Relative } \\
\text { Humidity (\%) }\end{array}$ & $\begin{array}{c}\text { Barometric } \\
\text { Pressure } \\
\text { (psi) }\end{array}$ & $\begin{array}{l}\text { Secondary } \\
\text { Dilution } \\
\text { Ratio }\end{array}$ & Test Date \\
\hline \multirow[t]{16}{*}{ Cummins, ISM 370, 2004} & E01920_02 & hot-start FTP & NBB Cand. & 2400 & - & - & 24 & $6 / 6 / 2007$ \\
\hline & E01920_03 & hot-start FTP & NBB Cand. & 2400 & - & - & 24 & $6 / 6 / 2007$ \\
\hline & E01920_04 & hot-start FTP & NBB Cand. & 2400 & - & - & 24 & $6 / 6 / 2007$ \\
\hline & E01921_03 & hot-start FTP & $\mathrm{B} 10$ & 2400 & - & - & 24 & $6 / 7 / 2007$ \\
\hline & E01921_04 & hot-start FTP & B10 & 2400 & - & - & 24 & $6 / 7 / 2007$ \\
\hline & E01926_03 & hot-start FTP & NBB Ref. & 2400 & - & - & 24 & $6 / 11 / 2007$ \\
\hline & E01926_04 & hot-start FTP & NBB Ref. & 2400 & - & - & 24 & $6 / 11 / 2007$ \\
\hline & E01927_02 & hot-start FTP & NBB-B05 & 2400 & - & - & 24 & $6 / 11 / 2007$ \\
\hline & E01927_03 & hot-start FTP & NBB-B05 & 2400 & - & - & 24 & $6 / 11 / 2007$ \\
\hline & E01927_04 & hot-start FTP & NBB-B05 & 2400 & - & - & 24 & $6 / 11 / 2007$ \\
\hline & E01928_02 & hot-start FTP & NBB-B05 & 2400 & - & - & 24 & $6 / 12 / 2007$ \\
\hline & E01928_03 & hot-start FTP & NBB-B05 & 2400 & - & - & 24 & $6 / 12 / 2007$ \\
\hline & E01928_04 & hot-start FTP & NBB-B05 & 2400 & - & - & 24 & $6 / 12 / 2007$ \\
\hline & E01929_02 & hot-start FTP & NBB Ref. & 2400 & - & - & 24 & $6 / 12 / 2007$ \\
\hline & E01929_03 & hot-start FTP & NBB Ref. & 2400 & - & - & 24 & $6 / 12 / 2007$ \\
\hline & E01929 04 & hot-start FTP & NBB Ref. & 2400 & - & - & 24 & $6 / 12 / 2007$ \\
\hline \multirow[t]{22}{*}{$\begin{array}{l}\text { Detriot Diesel, Rebuilt } \\
\text { DDC Series 60, } 1992\end{array}$} & E01941_02 & hot-start FTP & Grad. Ref. & 2400 & - & - & 24 & $6 / 19 / 2007$ \\
\hline & E01941_03 & hot-start FTP & Grad. Ref. & 2400 & - & - & 24 & $6 / 19 / 2007$ \\
\hline & E01941_04 & hot-start FTP & Grad. Ref. & 2400 & - & - & 24 & $6 / 19 / 2007$ \\
\hline & E01943_02 & hot-start FTP & Low Cetane & 2400 & - & - & 24 & $6 / 19 / 2007$ \\
\hline & E01943_03 & hot-start FTP & Low Cetane & 2400 & - & - & 24 & $6 / 19 / 2007$ \\
\hline & E01943_04 & hot-start FTP & Low Cetane & 2400 & - & - & 24 & $6 / 19 / 2007$ \\
\hline & E01944_01 & SET & Low Cetane & 2400 & - & - & 24 & $6 / 19 / 2007$ \\
\hline & E01947_03 & hot-start FTP & Low Cetane trt. 2 & 2400 & - & - & 24 & $6 / 20 / 2007$ \\
\hline & E01947_04 & hot-start FTP & Low Cetane trt. 2 & 2400 & - & - & 24 & $6 / 20 / 2007$ \\
\hline & E01948_01 & SET & Low Cetane trt. 2 & 2400 & - & - & 24 & $6 / 20 / 2007$ \\
\hline & E01949_02 & hot-start FTP & Low Cetane trt. 3 & 2400 & - & - & 24 & $6 / 20 / 2007$ \\
\hline & E01949_03 & hot-start FTP & Low Cetane trt. 3 & 2400 & - & - & 24 & $6 / 20 / 2007$ \\
\hline & E01949_04 & hot-start FTP & Low Cetane trt. 3 & 2400 & - & - & 24 & $6 / 20 / 2007$ \\
\hline & E01950_01 & SET & Low Cetane trt. 3 & 2400 & - & - & 24 & $6 / 20 / 2007$ \\
\hline & E01951_02 & hot-start FTP & Low Cetane trt. 4 & 2400 & - & - & 24 & $6 / 20 / 2007$ \\
\hline & E01951_03 & hot-start FTP & Low Cetane trt. 4 & 2400 & - & - & 24 & $6 / 20 / 2007$ \\
\hline & E01951_04 & hot-start FTP & Low Cetane trt. 4 & 2400 & - & - & 24 & $6 / 20 / 2007$ \\
\hline & E01952_01 & SET & Low Cetane trt. 4 & 2400 & - & - & 24 & $6 / 20 / 2007$ \\
\hline & E01953_03 & hot-start FTP & Low Cetane trt. 5 & 2400 & - & 14.253 & 24 & $6 / 21 / 2007$ \\
\hline & E01953_04 & hot-start FTP & Low Cetane trt. 5 & 2400 & - & 14.249 & 24 & $6 / 21 / 2007$ \\
\hline & E01954_01 & SET & Low Cetane trt. 5 & 2400 & - & 14.242 & 24 & $6 / 21 / 2007$ \\
\hline & E01955_02 & hot-start FTP & Low Cetane trt. 6 & 2400 & - & 14.232 & 24 & $6 / 21 / 2007$ \\
\hline
\end{tabular}




\begin{tabular}{|c|c|c|c|c|c|c|c|c|}
\hline & E01955_03 & hot-start FTP & Low Cetane trt. 6 & 2400 & - & 14.230 & 24 & $6 / 21 / 2007$ \\
\hline & E01955_04 & hot-start FTP & Low Cetane trt. 6 & 2400 & - & 14.220 & 24 & $6 / 21 / 2007$ \\
\hline & E01956_01 & SET & Low Cetane trt. 6 & 2400 & - & 14.212 & 24 & $6 / 21 / 2007$ \\
\hline & E01957_02 & hot-start FTP & Low Cetane trt. 7 & 2400 & - & 14.201 & 24 & $6 / 21 / 2007$ \\
\hline & E01957_03 & hot-start FTP & Low Cetane trt. 7 & 2400 & - & 14.194 & 24 & $6 / 21 / 2007$ \\
\hline & E01957_04 & hot-start FTP & Low Cetane trt. 7 & 2400 & - & 14.189 & 24 & $6 / 21 / 2007$ \\
\hline & E01958_01 & SET & Low Cetane trt. 7 & 2400 & - & 14.180 & 24 & $6 / 21 / 2007$ \\
\hline & E01959_02 & hot-start FTP & Low Cetane trt. 8 & 2400 & - & 14.218 & 24 & $6 / 22 / 2007$ \\
\hline & E01959_03 & hot-start FTP & Low Cetane trt. 8 & 2400 & - & 14.218 & 24 & $6 / 22 / 2007$ \\
\hline & E01959_04 & hot-start FTP & Low Cetane trt. 8 & 2400 & - & 14.220 & 24 & $6 / 22 / 2007$ \\
\hline & E01960_01 & SET & Low Cetane trt. 8 & 2400 & - & 14.221 & 24 & $6 / 22 / 2007$ \\
\hline & E01961_02 & hot-start FTP & Low Cetane trt. 9 & 2400 & - & 14.222 & 24 & $6 / 22 / 2007$ \\
\hline & E01961_03 & hot-start FTP & Low Cetane trt. 9 & 2400 & - & 14.222 & 24 & $6 / 22 / 2007$ \\
\hline & E01961_04 & hot-start FTP & Low Cetane trt. 9 & 2400 & - & 14.223 & 24 & $6 / 22 / 2007$ \\
\hline & E01962_01 & SET & Low Cetane trt. 9 & 2400 & - & 14.219 & 24 & $6 / 22 / 2007$ \\
\hline & E01963_02 & hot-start FTP & Low Cetane trt. 10 & 2400 & - & 14.207 & 24 & $6 / 22 / 2007$ \\
\hline & E01963_03 & hot-start FTP & Low Cetane trt. 10 & 2400 & - & 14.200 & 24 & $6 / 22 / 2007$ \\
\hline & E01963_04 & hot-start FTP & Low Cetane trt. 10 & 2400 & - & 14.191 & 24 & $6 / 22 / 2007$ \\
\hline & E01964_01 & SET & Low Cetane trt. 10 & 2400 & - & 14.184 & 24 & $6 / 22 / 2007$ \\
\hline \multirow[t]{29}{*}{$\begin{array}{c}\text { Detriot Diesel, DDC } \\
\text { Series 60, } 1992\end{array}$} & E01967_03 & hot-start FTP & 2007 CP Cert. & 2400 & - & 14.330 & 24 & $6 / 26 / 2007$ \\
\hline & E01967_04 & hot-start FTP & 2007 CP Cert. & 2400 & - & 14.343 & 24 & $6 / 26 / 2007$ \\
\hline & E01968_04 & hot-start FTP & 2007 CP Cert. & 2400 & - & 14.321 & 24 & $6 / 26 / 2007$ \\
\hline & E01968_05 & hot-start FTP & 2007 CP Cert. & 2400 & - & 14.315 & 24 & $6 / 26 / 2007$ \\
\hline & E01968_06 & hot-start FTP & 2007 CP Cert. & 2400 & - & 14.311 & 24 & $6 / 26 / 2007$ \\
\hline & E01972_02 & hot-start FTP & RF 71 & 2400 & - & 14.276 & 24 & $6 / 27 / 2007$ \\
\hline & E01972_03 & hot-start FTP & RF 71 & 2400 & - & 14.272 & 24 & $6 / 27 / 2007$ \\
\hline & E01972_04 & hot-start FTP & RF 71 & 2400 & - & 14.270 & 24 & $6 / 27 / 2007$ \\
\hline & E01976_03 & hot-start FTP & CF 16 trt. 4 & 2400 & - & 14.242 & 24 & $6 / 28 / 2007$ \\
\hline & E01976_04 & hot-start FTP & CF 16 trt. 4 & 2400 & - & 14.226 & 24 & $6 / 28 / 2007$ \\
\hline & E01976_05 & hot-start FTP & CF 16 trt. 4 & 2400 & - & 14.222 & 24 & $6 / 28 / 2007$ \\
\hline & E01977_03 & hot-start FTP & RF 71 & 2400 & - & 14.147 & 24 & $6 / 28 / 2007$ \\
\hline & E01977_04 & hot-start FTP & RF 71 & 2400 & - & 14.182 & 24 & $6 / 28 / 2007$ \\
\hline & E01977_05 & hot-start FTP & RF 71 & 2400 & - & 14.180 & 24 & $6 / 28 / 2007$ \\
\hline & E02097_01 & warm-start FTP & TCEQ Tex LED Ref. & 2400 & - & 14.153 & 24 & $8 / 24 / 2007$ \\
\hline & E02097_02 & hot-start FTP & TCEQ Tex LED Ref. & 2400 & - & 14.147 & 24 & $8 / 24 / 2007$ \\
\hline & E02097_03 & hot-start FTP & TCEQ Tex LED Ref. & 2400 & - & 14.144 & 24 & $8 / 24 / 2007$ \\
\hline & E02099_02 & hot-start FTP & TCEQ Tex LED Ref. & 2400 & - & 14.152 & 24 & $8 / 25 / 2007$ \\
\hline & E02099_03 & hot-start FTP & TCEQ Tex LED Ref. & 2400 & - & 14.152 & 24 & $8 / 25 / 2007$ \\
\hline & E02099_04 & hot-start FTP & TCEQ Tex LED Ref. & 2400 & - & 14.154 & 24 & $8 / 25 / 2007$ \\
\hline & E02099_05 & hot-start FTP & TCEQ Tex LED Ref. & 2400 & - & 14.155 & 24 & $8 / 25 / 2007$ \\
\hline & E02101_02 & hot-start FTP & Candidate & 2400 & - & 14.198 & 24 & $8 / 26 / 2007$ \\
\hline & E02101_03 & hot-start FTP & Candidate & 2400 & - & 14.207 & 24 & $8 / 26 / 2007$ \\
\hline & E02101_04 & hot-start FTP & Candidate & 2400 & - & 14.208 & 24 & $8 / 26 / 2007$ \\
\hline & E02101_05 & hot-start FTP & Candidate & 2400 & - & 14.208 & 24 & $8 / 26 / 2007$ \\
\hline & E02103_04 & hot-start FTP & Candidate & 2400 & - & 14.254 & 24 & $8 / 27 / 2007$ \\
\hline & E02103_05 & hot-start FTP & Candidate & 2400 & - & 14.251 & 24 & $8 / 27 / 2007$ \\
\hline & E02103_06 & hot-start FTP & Candidate & 2400 & - & 14.249 & 24 & $8 / 27 / 2007$ \\
\hline & E02103_07 & hot-start FTP & Candidate & 2400 & - & 14.247 & 24 & $8 / 27 / 2007$ \\
\hline
\end{tabular}




\begin{tabular}{|c|c|c|c|c|c|c|c|c|}
\hline & E02107_01 & warm-start FTP & TCEQ Tex LED Ref. & 2400 & - & 14.244 & 24 & $8 / 28 / 2007$ \\
\hline & E02107_02 & hot-start FTP & TCEQ Tex LED Ref. & 2400 & - & 14.242 & 24 & $8 / 28 / 2007$ \\
\hline & E02107_03 & hot-start FTP & TCEQ Tex LED Ref. & 2400 & - & 14.243 & 24 & $8 / 28 / 2007$ \\
\hline & E02107_04 & hot-start FTP & TCEQ Tex LED Ref. & 2400 & - & 14.242 & 24 & $8 / 28 / 2007$ \\
\hline \multirow[t]{43}{*}{$\begin{array}{l}\text { Detriot Diesel, Rebuilt } \\
\text { DDC Series 60, } 1992\end{array}$} & E02123_02 & hot-start FTP & PONCA & 2400 & - & 14.217 & 24 & 9/10/2007 \\
\hline & E02123_03 & hot-start FTP & PONCA & 2400 & - & 14.222 & 24 & 9/10/2007 \\
\hline & E02124_01 & hot-start FTP & PONCA trt. 1 & 2400 & - & 14.221 & 24 & 9/10/2007 \\
\hline & E02124_02 & hot-start FTP & PONCA trt. 1 & 2400 & - & 14.215 & 24 & 9/10/2007 \\
\hline & E02125_02 & hot-start FTP & Trainer & 2400 & - & 14.203 & 24 & 9/10/2007 \\
\hline & E02125_03 & hot-start FTP & Trainer & 2400 & - & 14.198 & 24 & 9/10/2007 \\
\hline & E02126_01 & hot-start FTP & 50/50 Trainer/Cand. & 2400 & - & 14.193 & 24 & $9 / 10 / 2007$ \\
\hline & E02126_02 & hot-start FTP & 50/50 Trainer/Cand. & 2400 & - & 14.181 & 24 & $9 / 10 / 2007$ \\
\hline & E02127_01 & hot-start FTP & 50/50 Trainer/Cand. trt. 1 & 2400 & - & 14.175 & 24 & 9/10/2007 \\
\hline & E02127_02 & hot-start FTP & 50/50 Trainer/Cand. trt. 1 & 2400 & - & 14.168 & 24 & 9/10/2007 \\
\hline & E02136_02 & hot-start FTP & Low Cetane & 2400 & - & 14.208 & 24 & 9/12/2007 \\
\hline & E02136_03 & hot-start FTP & Low Cetane & 2400 & - & 14.215 & 24 & $9 / 12 / 2007$ \\
\hline & E02136_04 & hot-start FTP & Low Cetane & 2400 & - & 14.221 & 24 & $9 / 12 / 2007$ \\
\hline & E02137_01 & SET & Low Cetane & 2400 & - & 14.225 & 24 & 9/12/2007 \\
\hline & E02138_02 & hot-start FTP & Low Cetane B10A & 2400 & - & 14.234 & 24 & $9 / 12 / 2007$ \\
\hline & E02138_03 & hot-start FTP & Low Cetane B10A & 2400 & - & 14.233 & 24 & 9/12/2007 \\
\hline & E02138_04 & hot-start FTP & Low Cetane B10A & 2400 & - & 14.231 & 24 & $9 / 12 / 2007$ \\
\hline & E02139_01 & SET & Low Cetane B10A & 2400 & - & 14.227 & 24 & 9/12/2007 \\
\hline & E02140_02 & hot-start FTP & Low Cetane B20A & 2400 & - & 14.225 & 24 & 9/12/2007 \\
\hline & E02140_03 & hot-start FTP & Low Cetane B20A & 2400 & - & 14.225 & 24 & $9 / 12 / 2007$ \\
\hline & E02140_04 & hot-start FTP & Low Cetane B20A & 2400 & - & 14.224 & 24 & $9 / 12 / 2007$ \\
\hline & E02141_01 & SET & Low Cetane B20A & 2400 & - & 14.225 & 24 & $9 / 12 / 2007$ \\
\hline & E02195_03 & hot-start FTP & FEM trt. 3 & 2400 & - & 14.203 & 24 & $10 / 17 / 2007$ \\
\hline & E02195_04 & hot-start FTP & FEM trt. 3 & 2400 & - & 14.195 & 24 & $10 / 17 / 2007$ \\
\hline & E02195_05 & hot-start FTP & FEM trt. 3 & 2400 & - & 14.188 & 24 & $10 / 17 / 2007$ \\
\hline & E02198_05 & hot-start FTP & FEM trt. 6 & 2400 & - & 14.025 & 24 & $10 / 19 / 2007$ \\
\hline & E02198_06 & hot-start FTP & FEM trt. 6 & 2400 & - & 14.020 & 24 & $10 / 19 / 2007$ \\
\hline & E02198_07 & hot-start FTP & FEM trt. 6 & 2400 & - & 14.016 & 24 & $10 / 19 / 2007$ \\
\hline & E02199_03 & hot-start FTP & FEM trt. 7 & 2400 & - & 14.001 & 24 & $10 / 19 / 2007$ \\
\hline & E02199_04 & hot-start FTP & FEM trt. 7 & 2400 & - & 14.095 & 24 & 10/19/2007 \\
\hline & E02199_05 & hot-start FTP & FEM trt. 7 & 2400 & - & 14.989 & 24 & 10/19/2007 \\
\hline & E02204_01 & SET & Guttman & 2400 & 37 & 14.132 & 24 & $10 / 24 / 2007$ \\
\hline & E02204_02 & SET & Guttman & 2400 & 37 & 14.142 & 24 & $10 / 24 / 2007$ \\
\hline & E02204_03 & SET & Guttman & 2400 & 37 & 14.148 & 24 & $10 / 24 / 2007$ \\
\hline & E02205_01 & SET & Guttman & 2400 & 88 & 14.156 & 24 & $10 / 24 / 2007$ \\
\hline & E02205_02 & SET & Guttman & 2400 & 88 & 14.163 & 24 & $10 / 24 / 2007$ \\
\hline & E02205_03 & SET & Guttman & 2400 & 88 & 14.174 & 24 & $10 / 24 / 2007$ \\
\hline & E02205_04 & SET & Guttman & 2400 & 88 & 14.195 & 24 & $10 / 24 / 2007$ \\
\hline & E02206_01 & SET & Guttman & 2400 & 60 & 14.193 & 24 & $10 / 24 / 2007$ \\
\hline & E02206_02 & SET & Guttman & 2400 & 60 & 14.189 & 24 & $10 / 24 / 2007$ \\
\hline & E02206_03 & SET & Guttman & 2400 & 60 & 14.187 & 24 & $10 / 24 / 2007$ \\
\hline & E02208_01 & SET & Guttman & 3400 & 50 & 14.264 & 24 & $10 / 24 / 2007$ \\
\hline & E02208_02 & SET & Guttman & 1400 & 50 & 14.255 & 24 & $10 / 24 / 2007$ \\
\hline
\end{tabular}




\section{A.2 DAY-TO-DAY VARIATION}

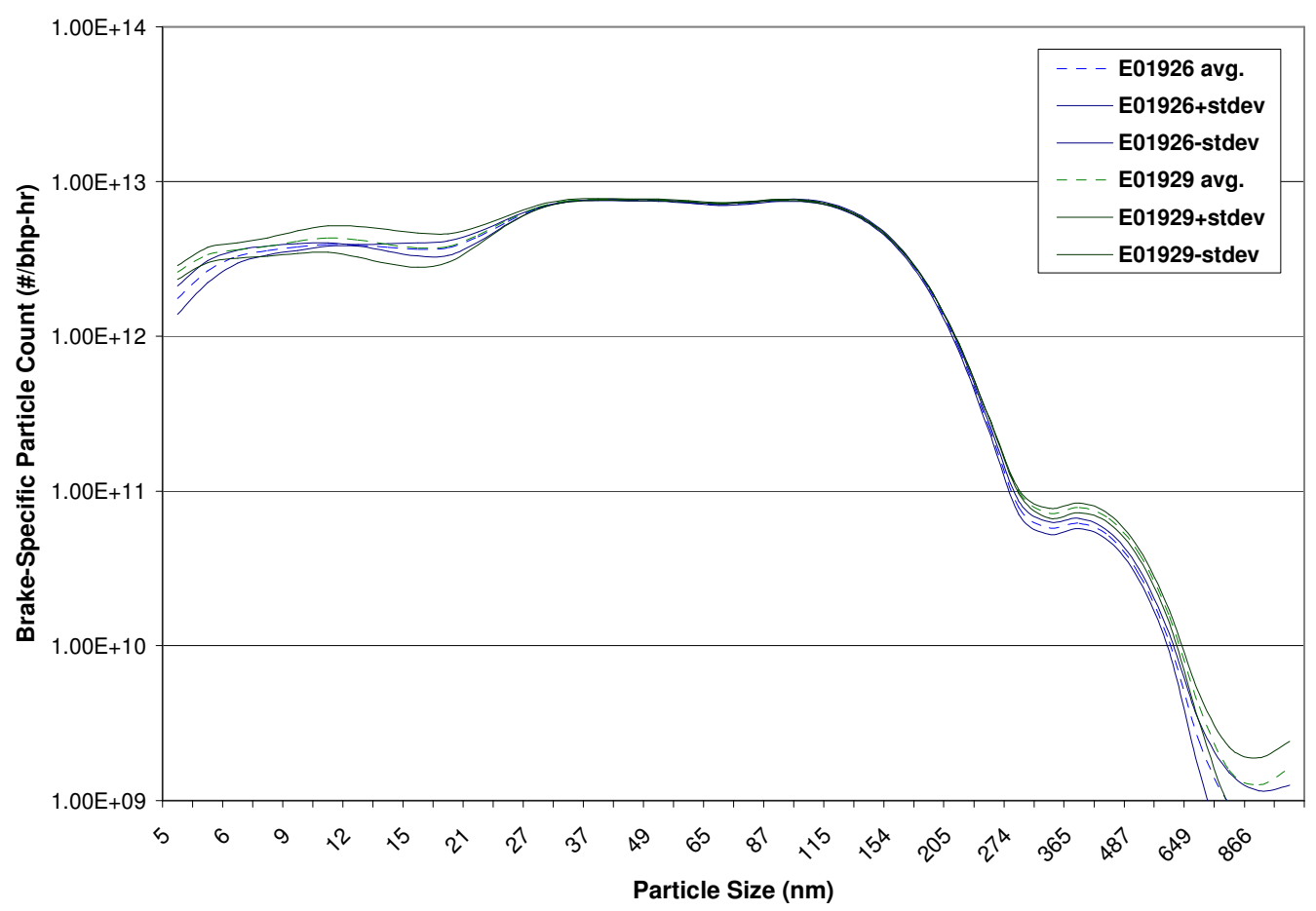

Figure A-1 Particle Concentration Comparison of E01926 and E01929 Test Sequences

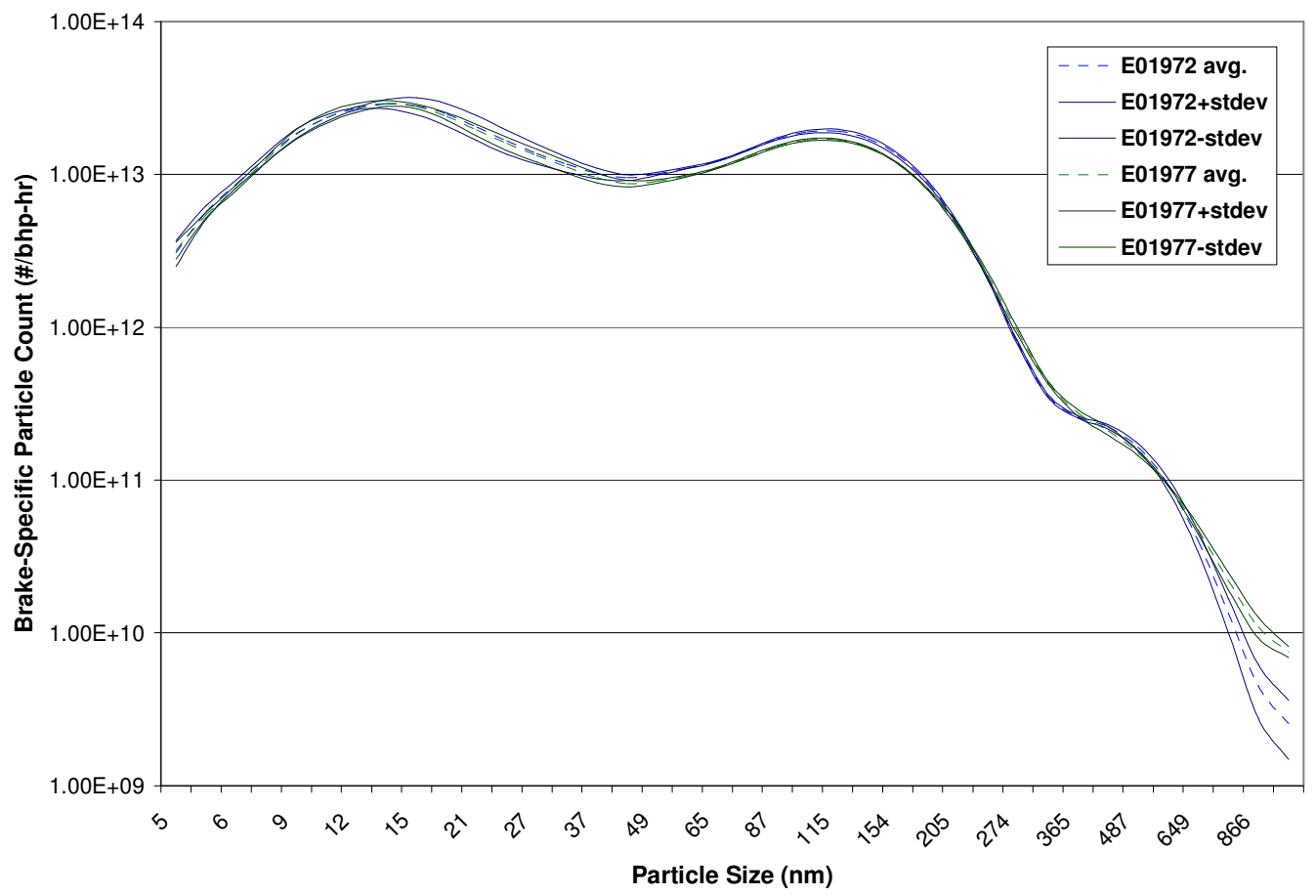

Figure A-2 Particle Concentration Comparison of E01972 and E01977 Test Sequences 


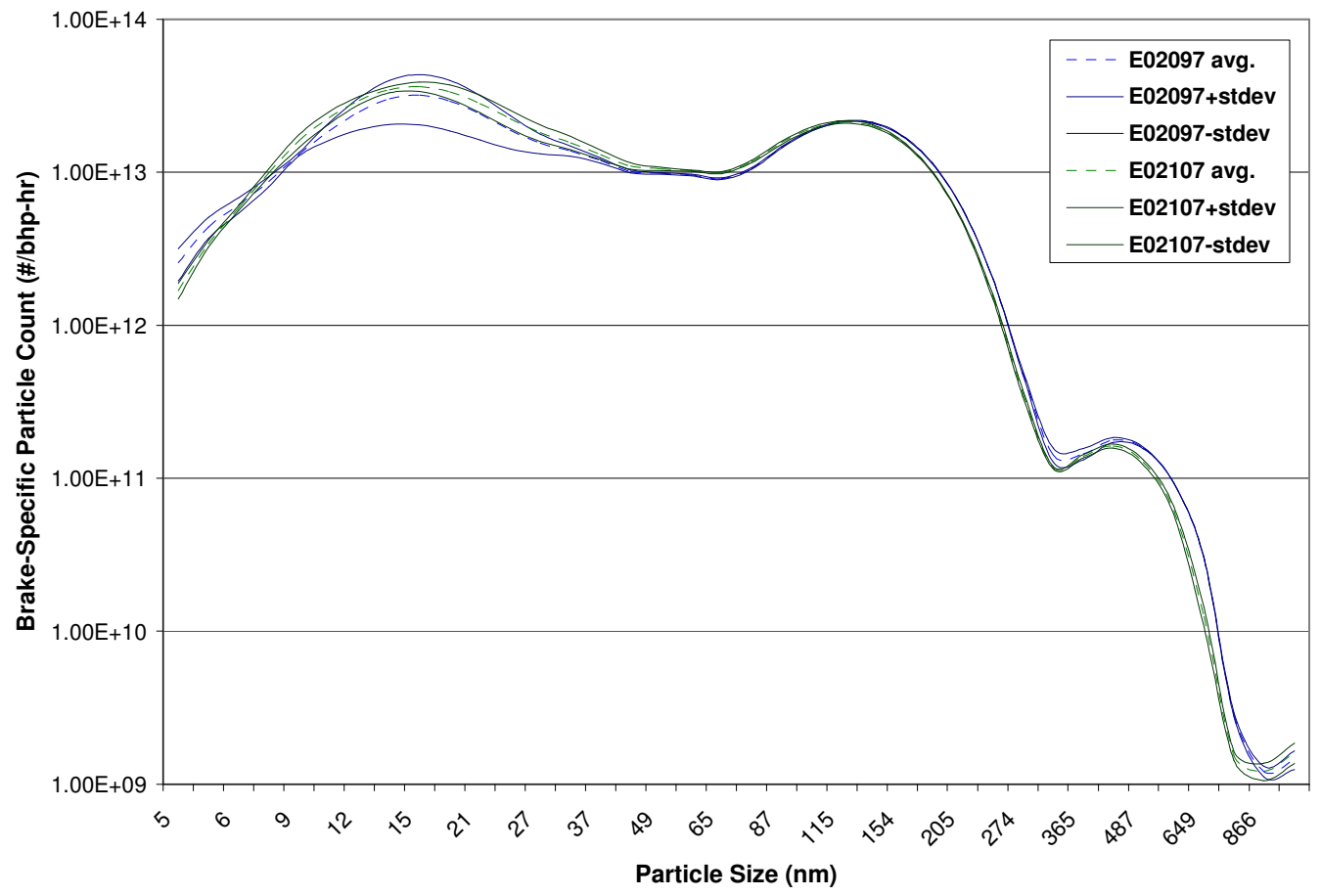

Figure A-3 Particle Concentration Comparison of E02097 and E02107 Test Sequences

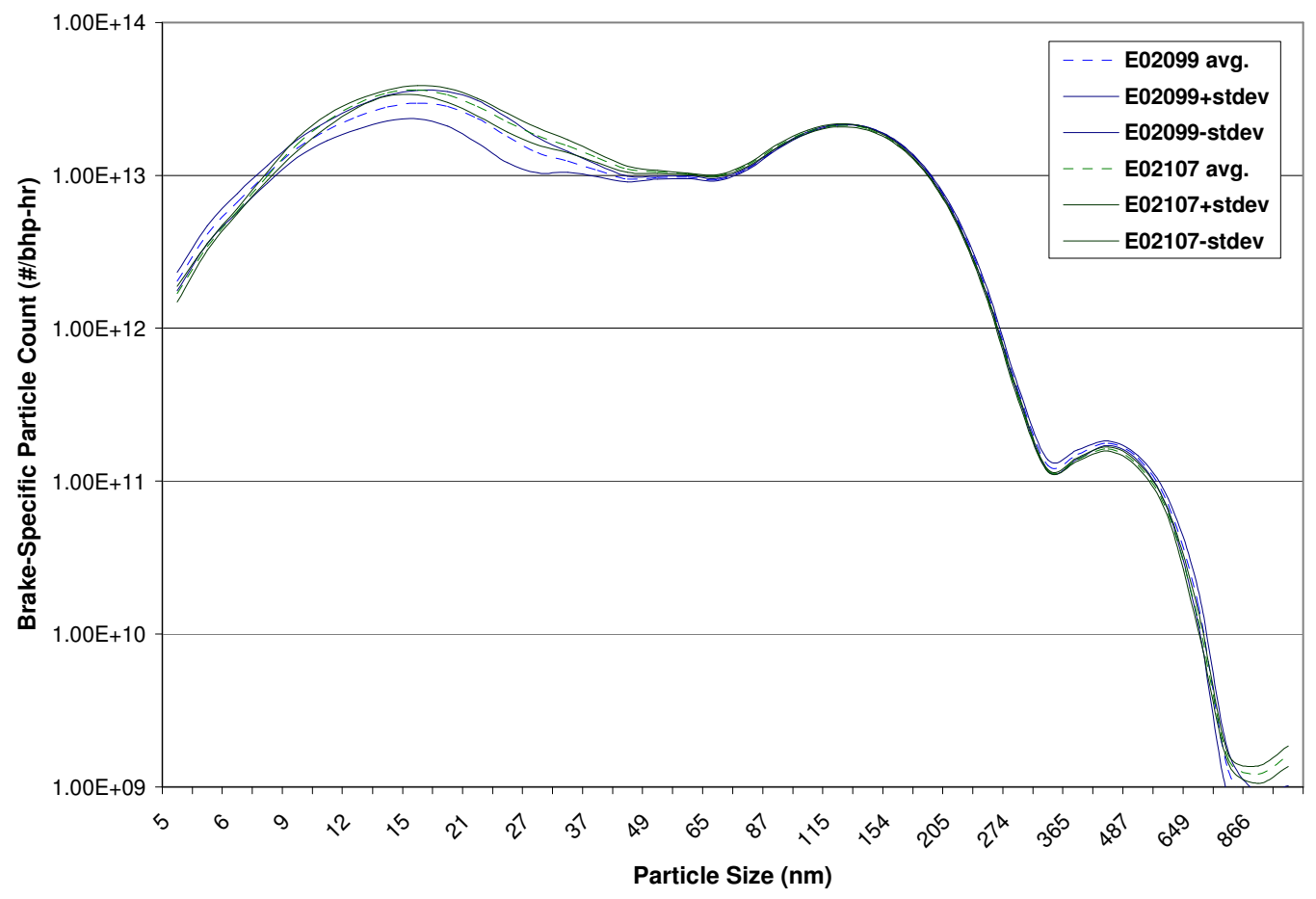

Figure A-4 Particle Concentration Comparison of E02099 and E02107 Test Sequences 


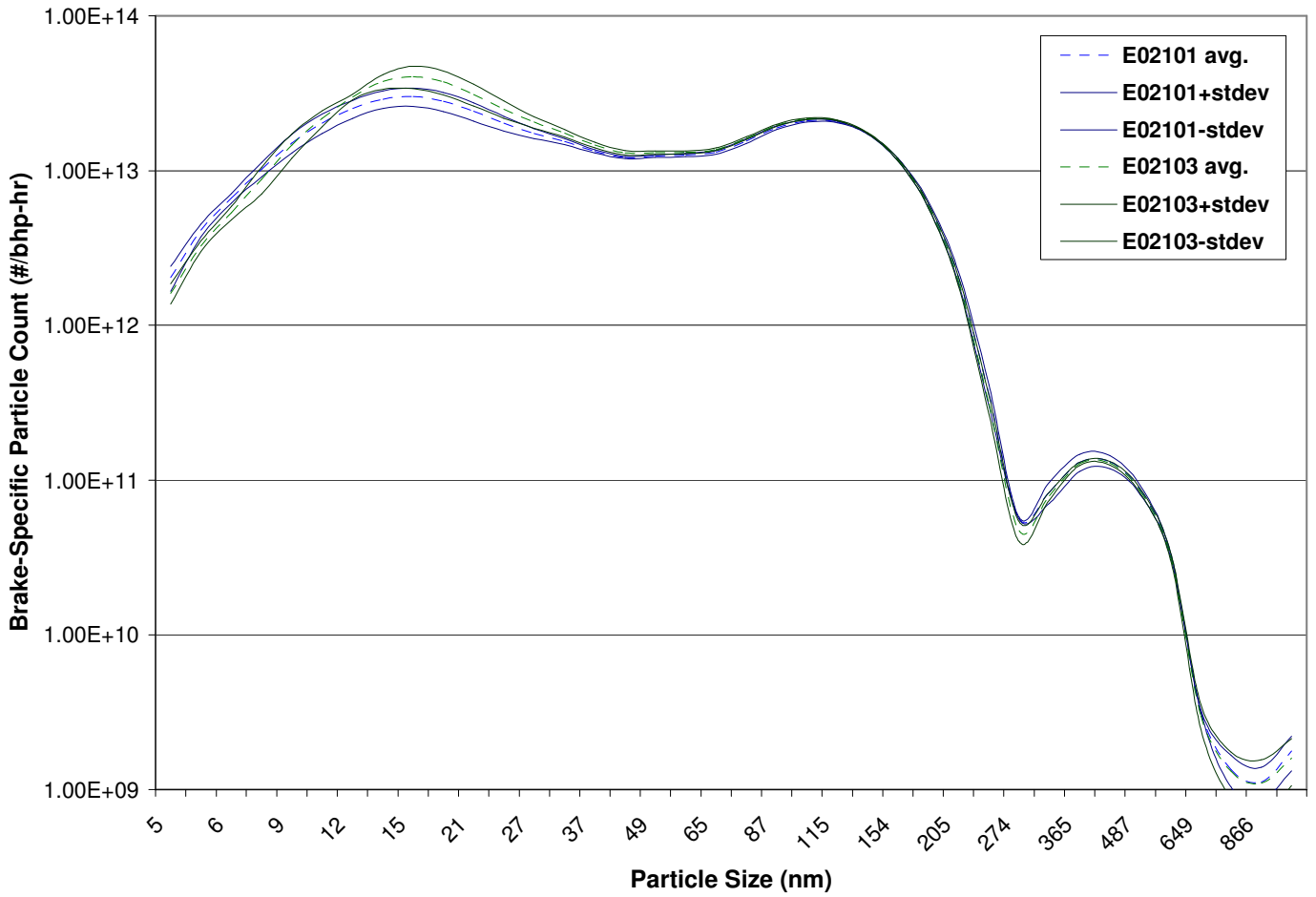

Figure A-5 Particle Concentration Comparison of E02101 and E02103 Test Sequences 


\section{A.3 BASE FUELS VS. ADDITIZED FUELS}

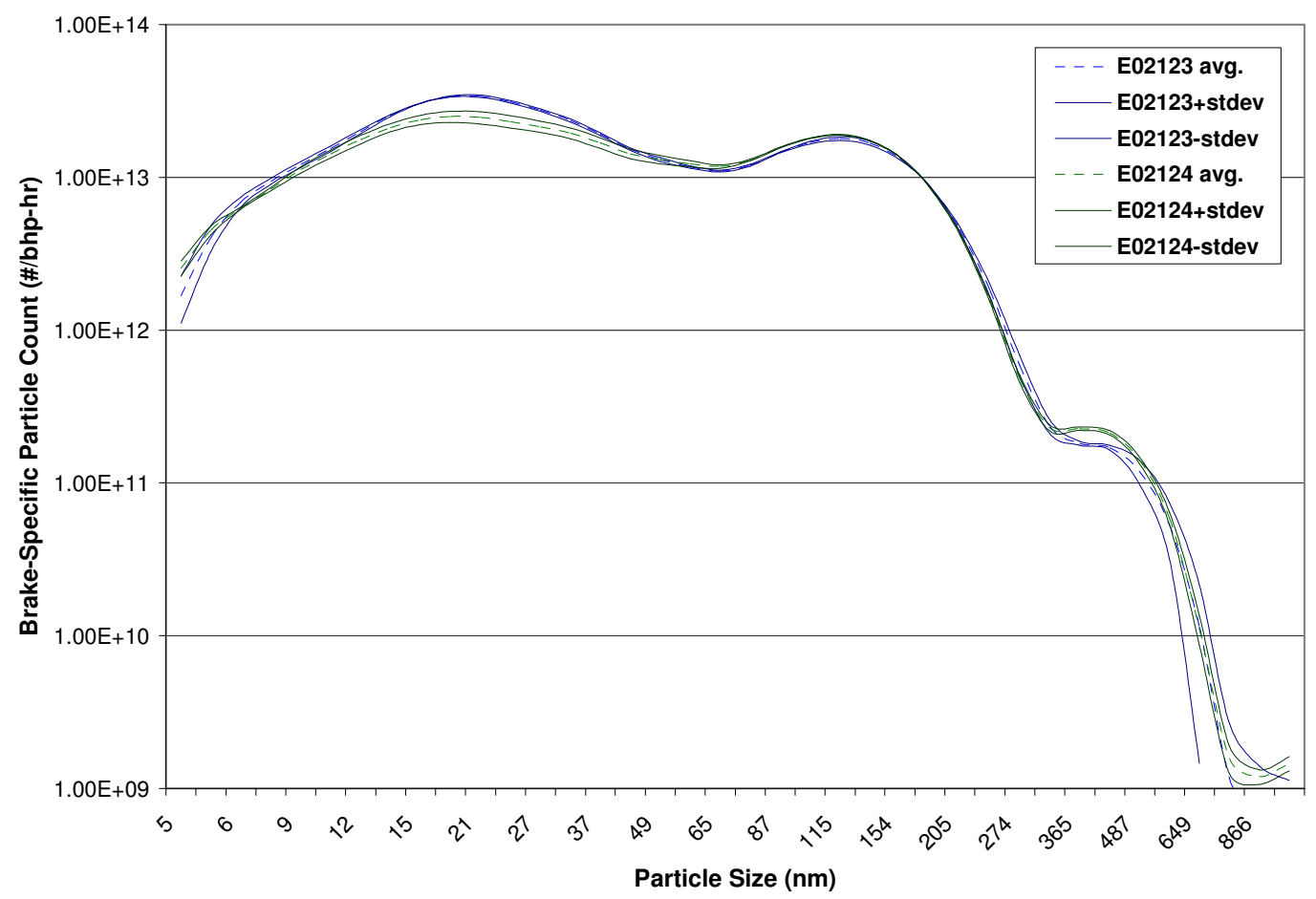

Figure A-6 Particle Concentration Comparison of E02123 and E02124 Test Sequences

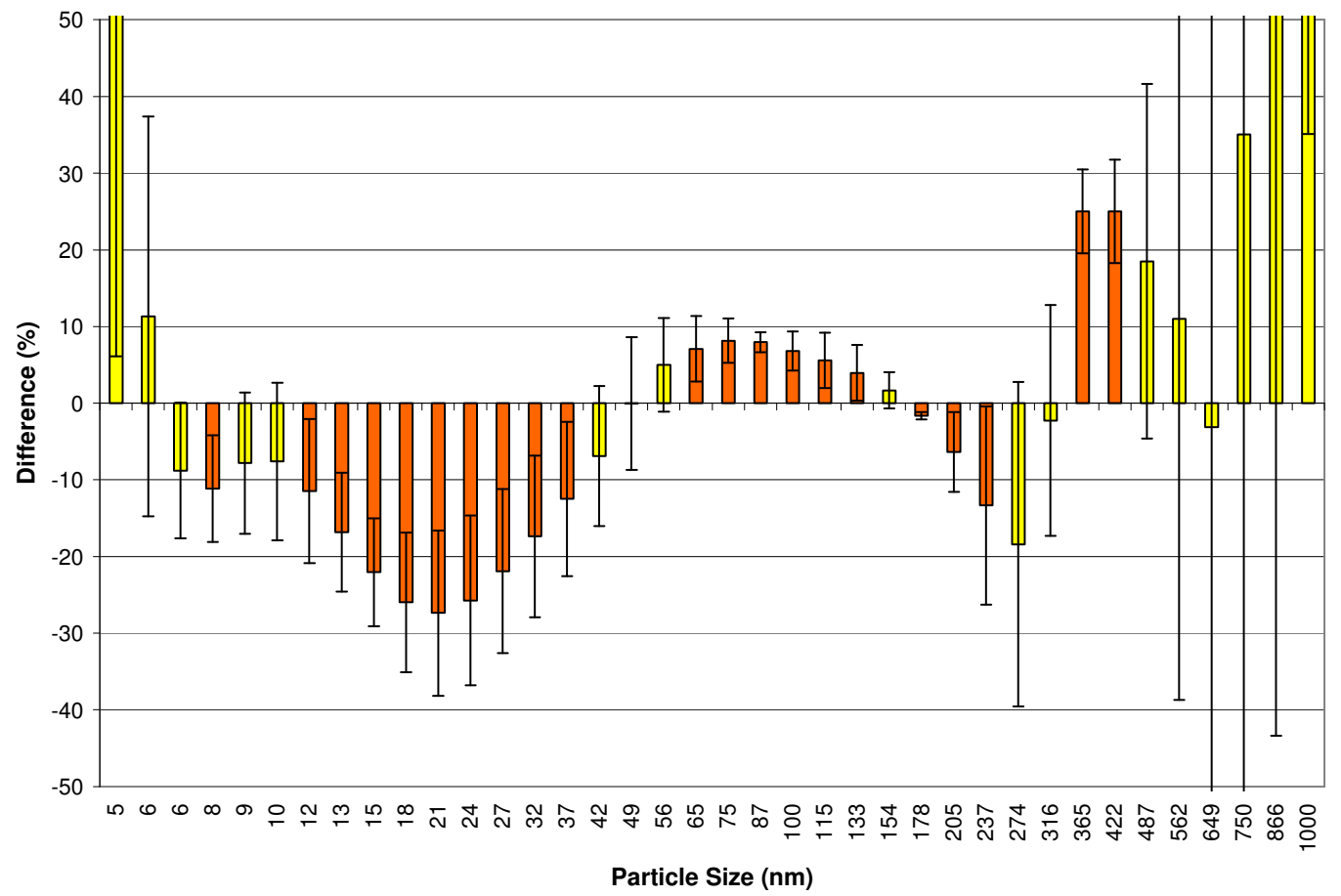

Figure A-7 Particle Concentration Differences between E02123 and E02124 Test Sequences 


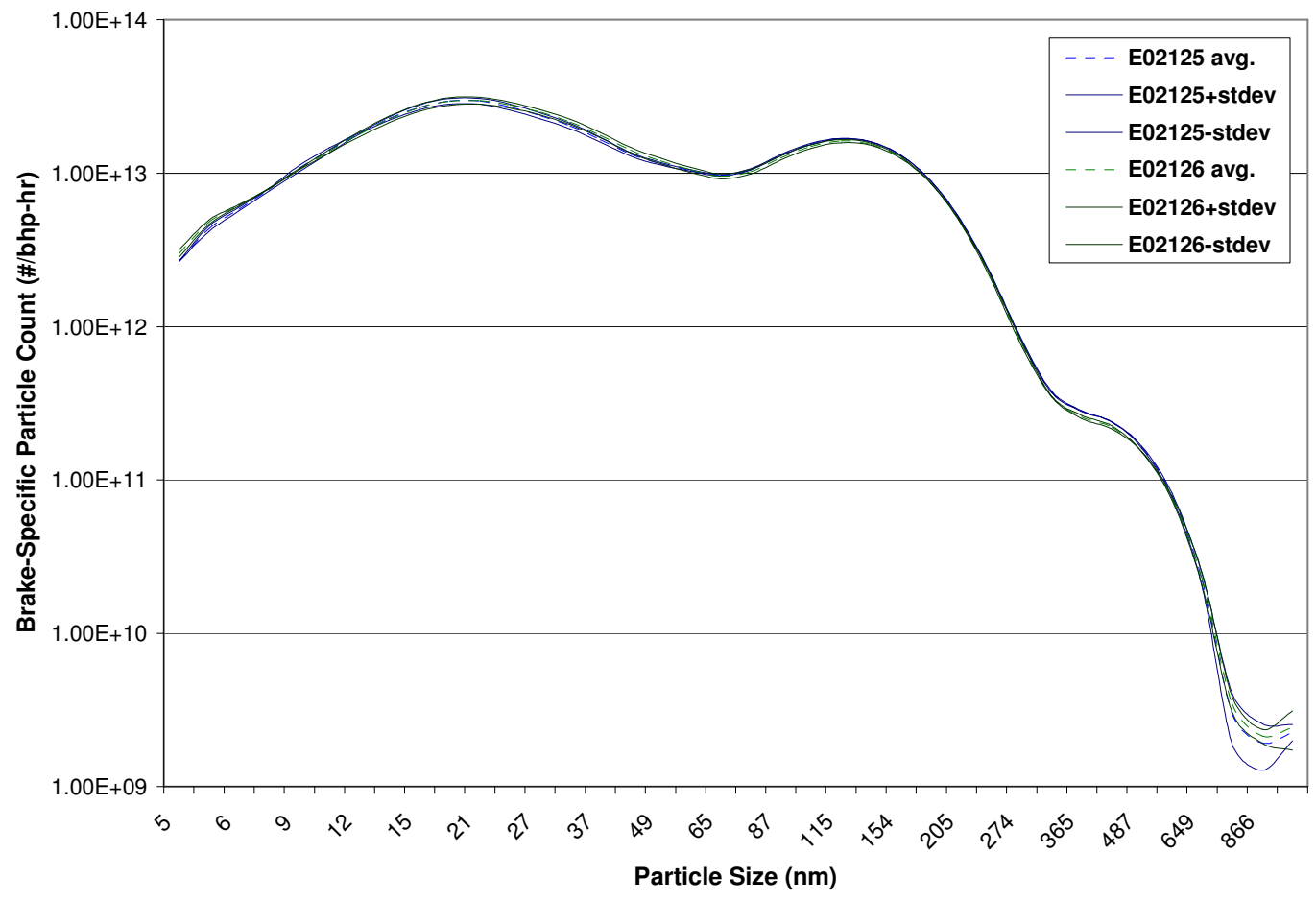

Figure A-8 Particle Concentration Comparison of E02125 and E02126 Test Sequences

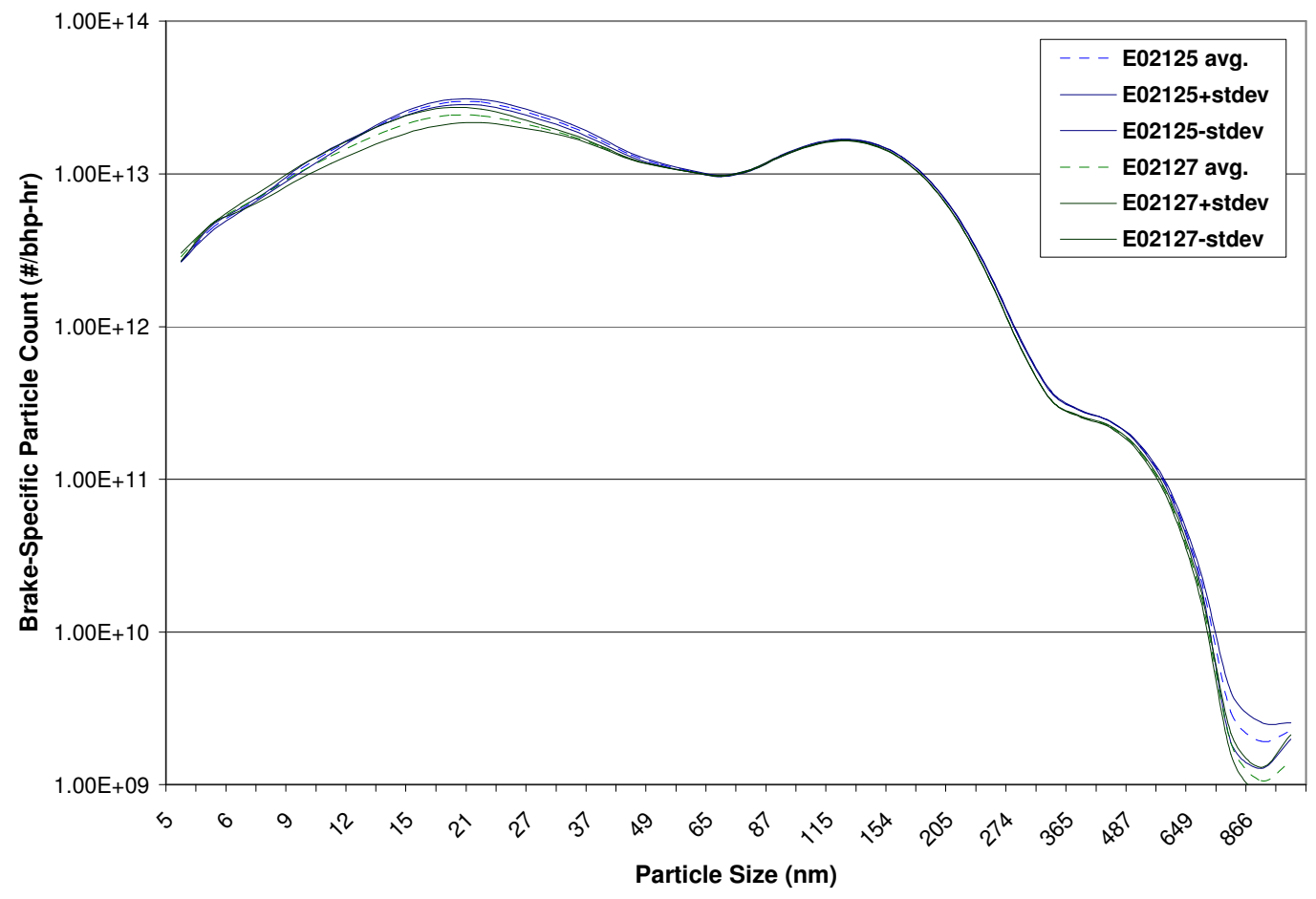

Figure A-9 Particle Concentration Comparison of E02125 and E02127 Test Sequences 


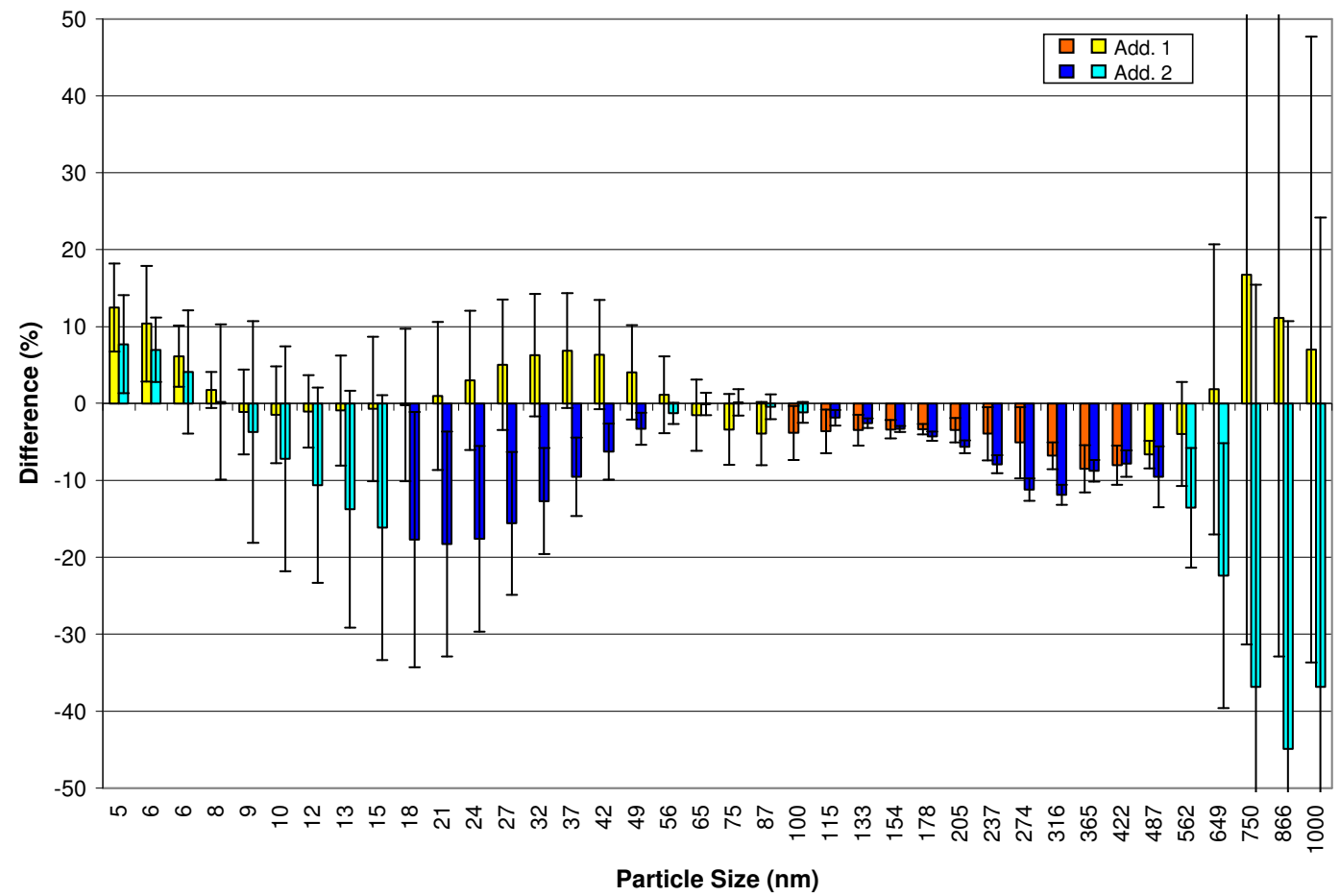

Figure A-10 Particle Concentration Differences between E02125, E02126, and E02127 Test Sequences

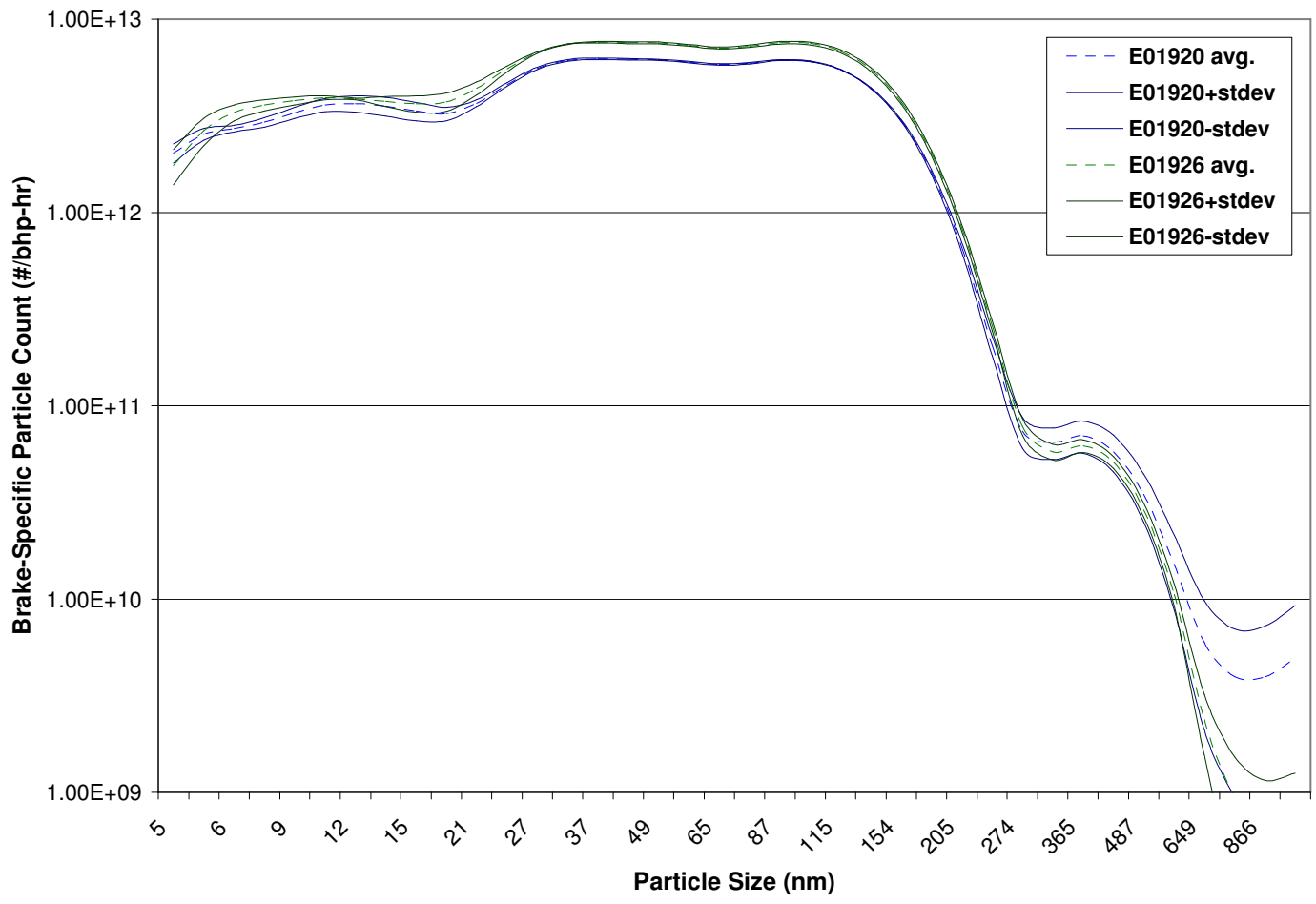

Figure A-11 Particle Concentration Comparison of E01920 and E01926 Test Sequences 


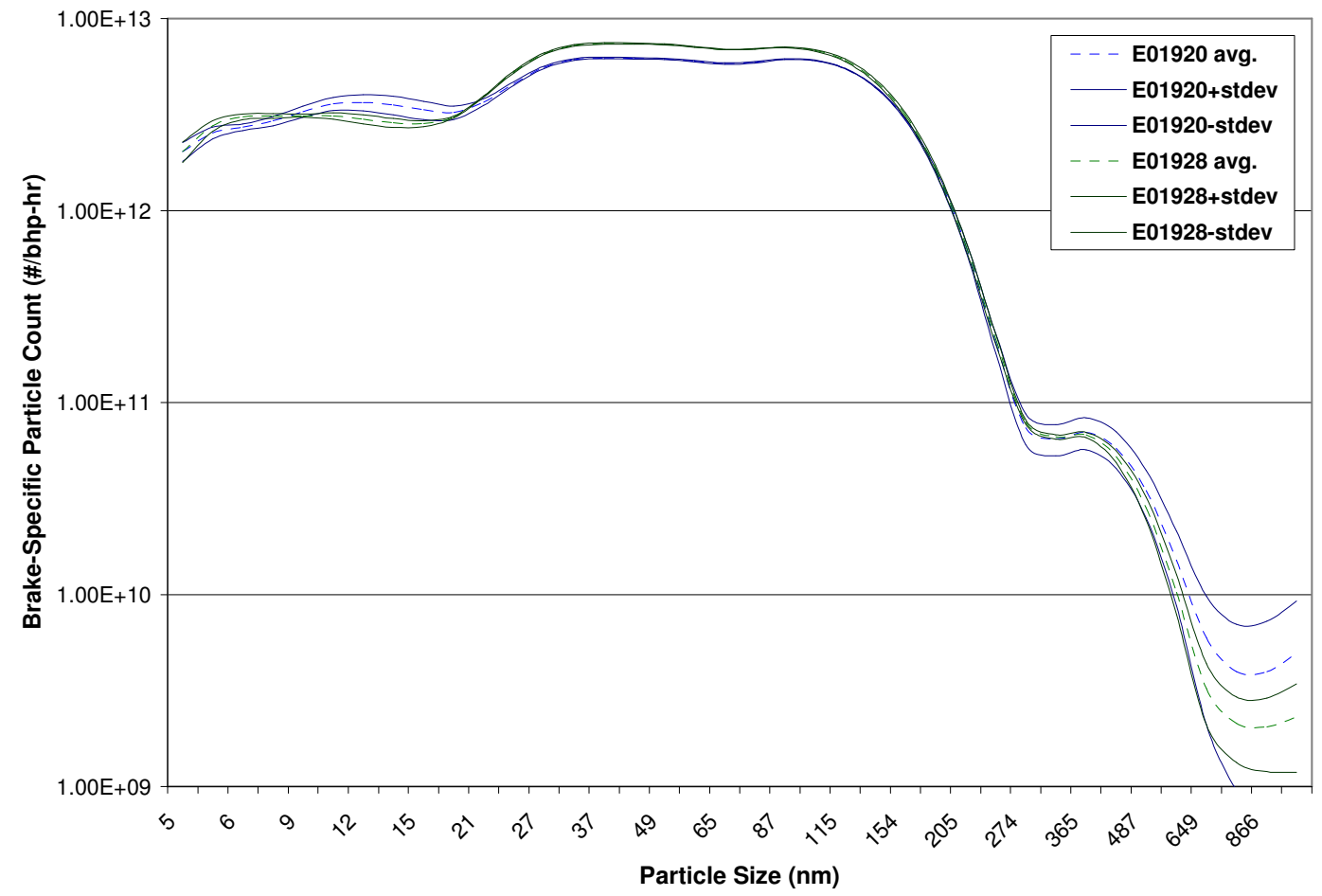

Figure A-12 Particle Concentration Comparison of E01920 and E01928 Test Sequences

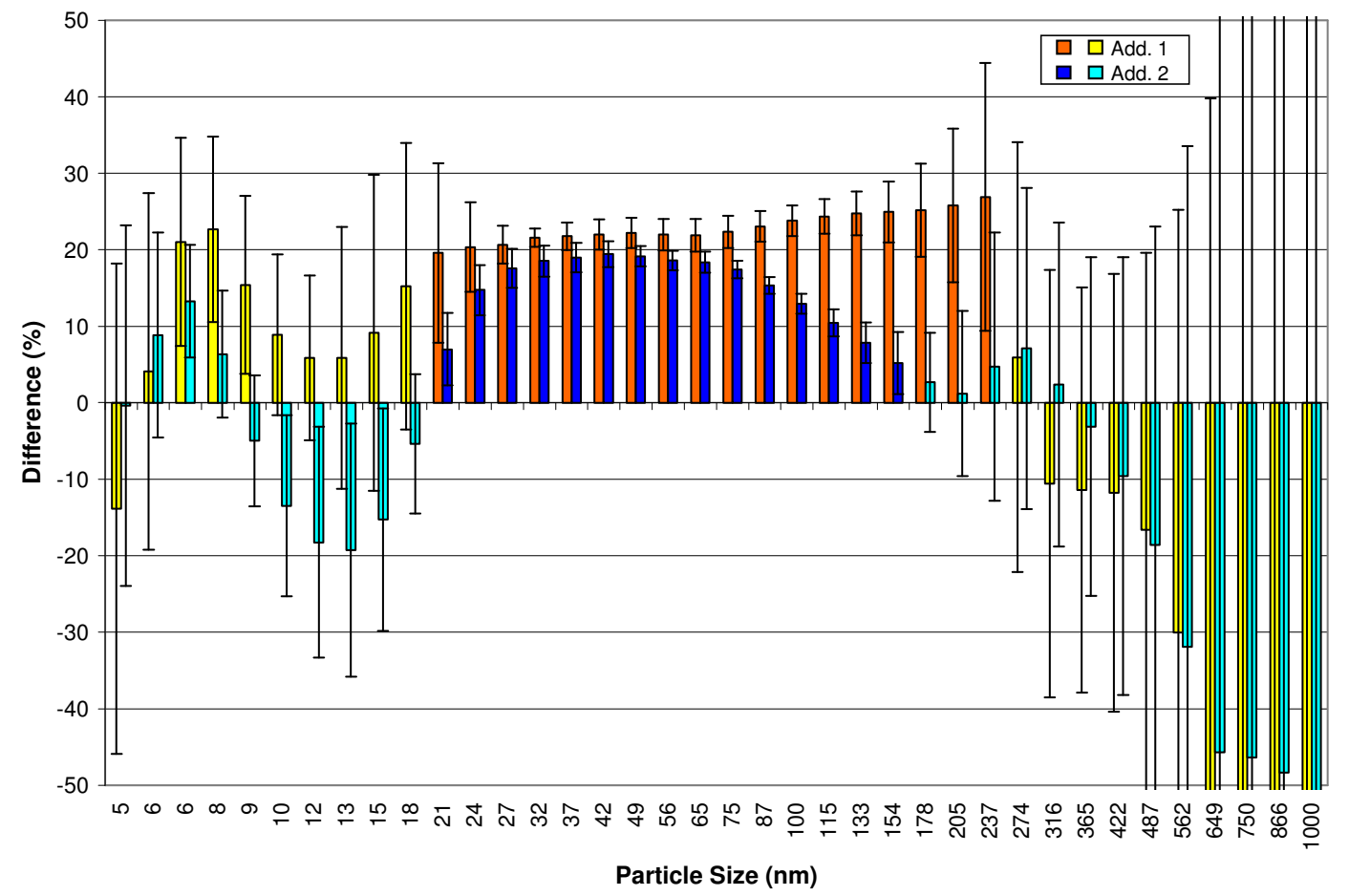

Figure A-13 Particle Concentration Differences between E01920, E01926, and E01928 Test Sequences 


\section{A.4 EXTREMES OF RUN-TO-RUN VARIATION}

\section{A.4.1 Best Repeatability for ISM 370}

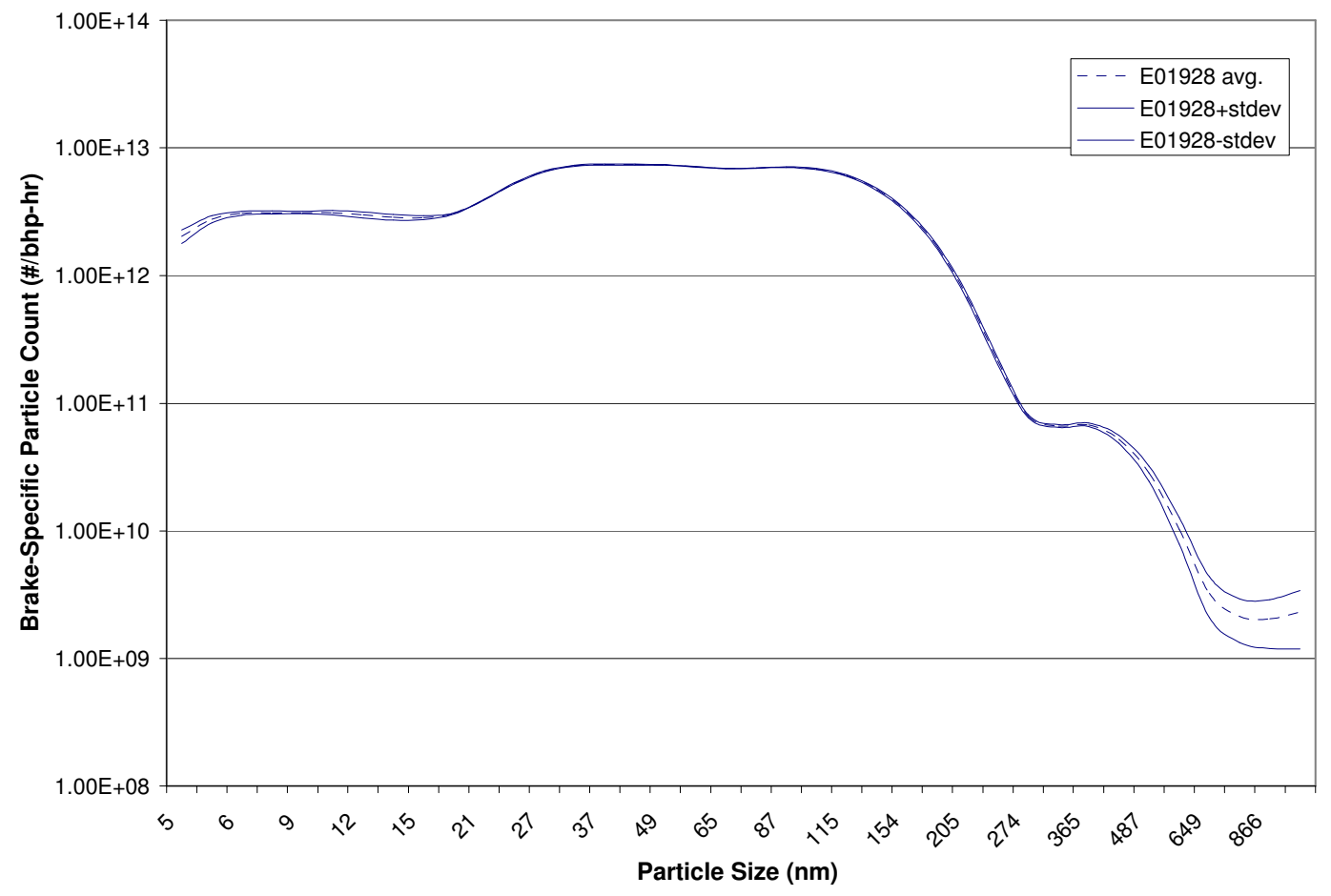

Figure A-14 Average Particle Concentrations of Test Sequence E01928 


\section{A.4.2 Worst Repeatability for ISM 370}

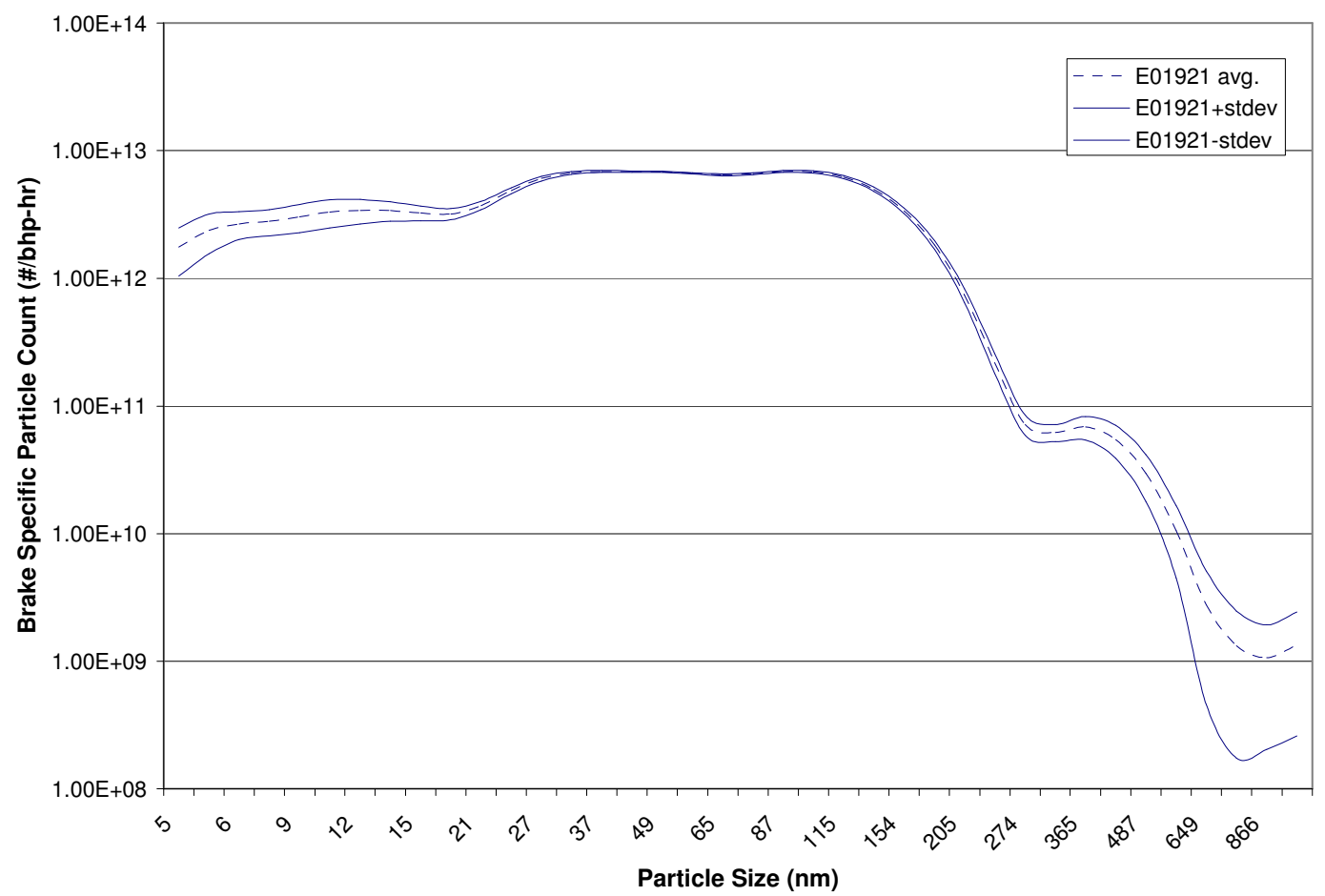

Figure A-15 Average Particle Concentrations of Test Sequence E01921 


\section{A.4.3 Best Repeatability for DDC S60}

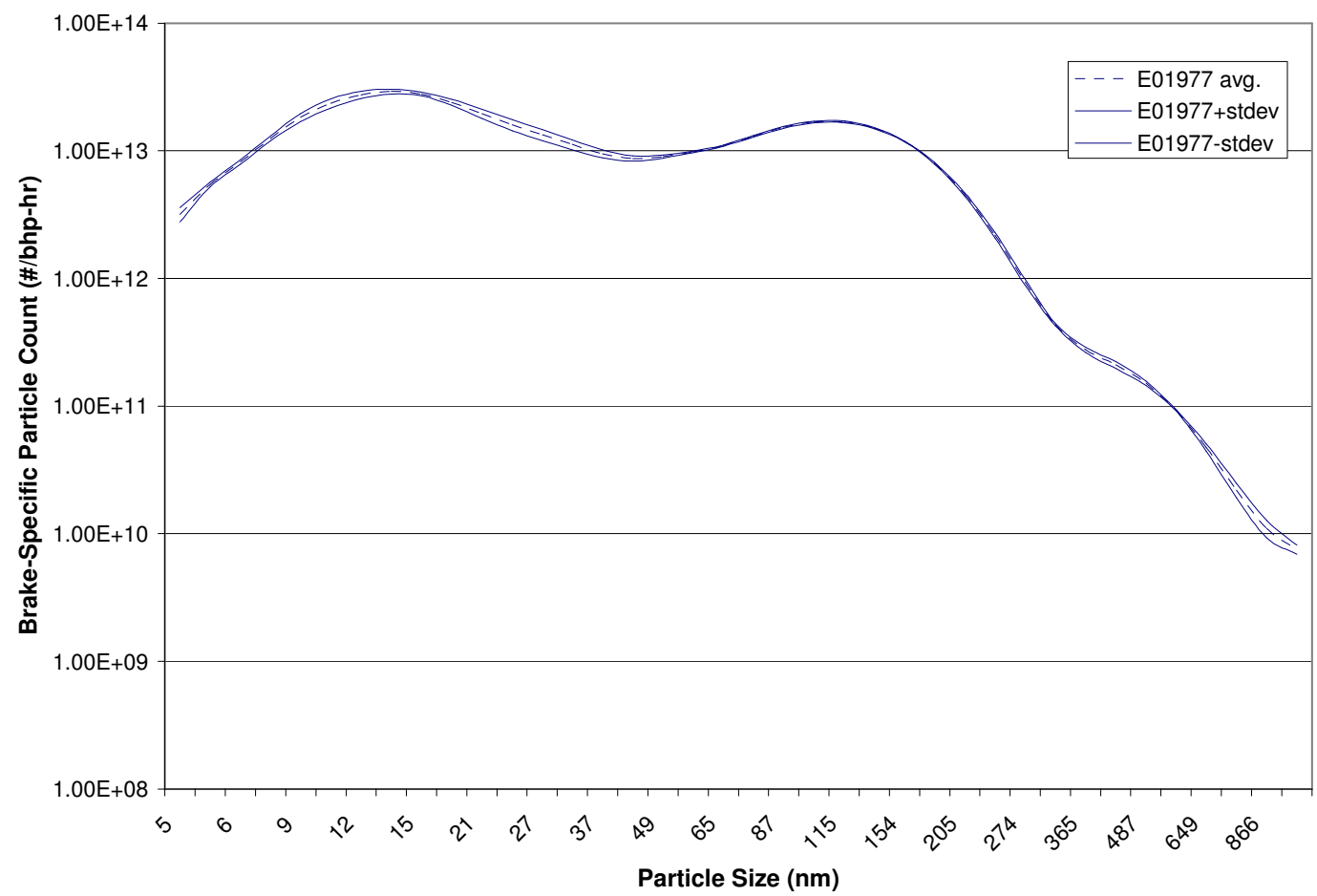

Figure A-16 Average Particle Concentrations of Test Sequence E01977 


\section{A.4.4 Worst Repeatability for DDC S60}

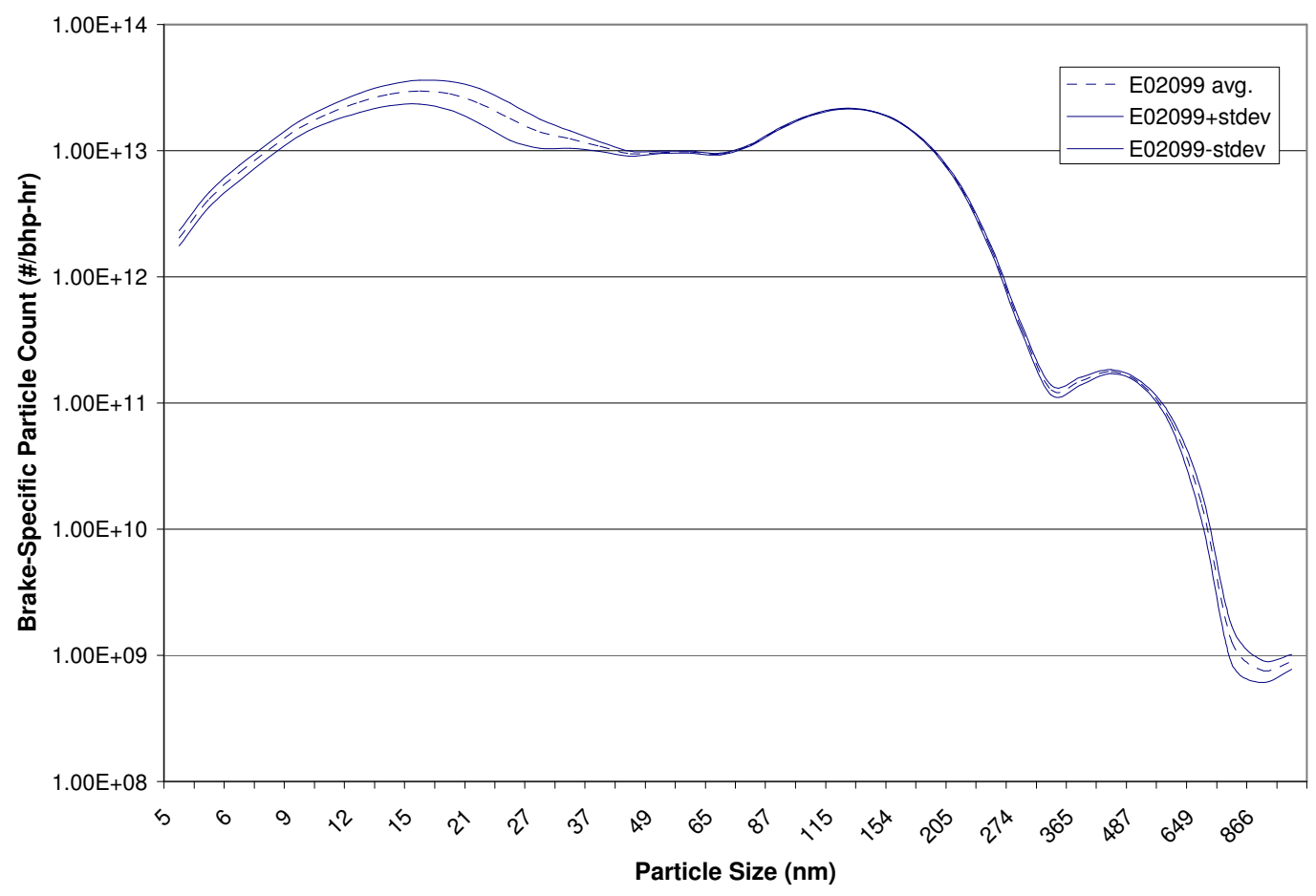

Figure A-17 Average Particle Concentrations of Test Sequence E02099 


\section{A.4.5 Best Repeatability for Rebuilt DDC S60}

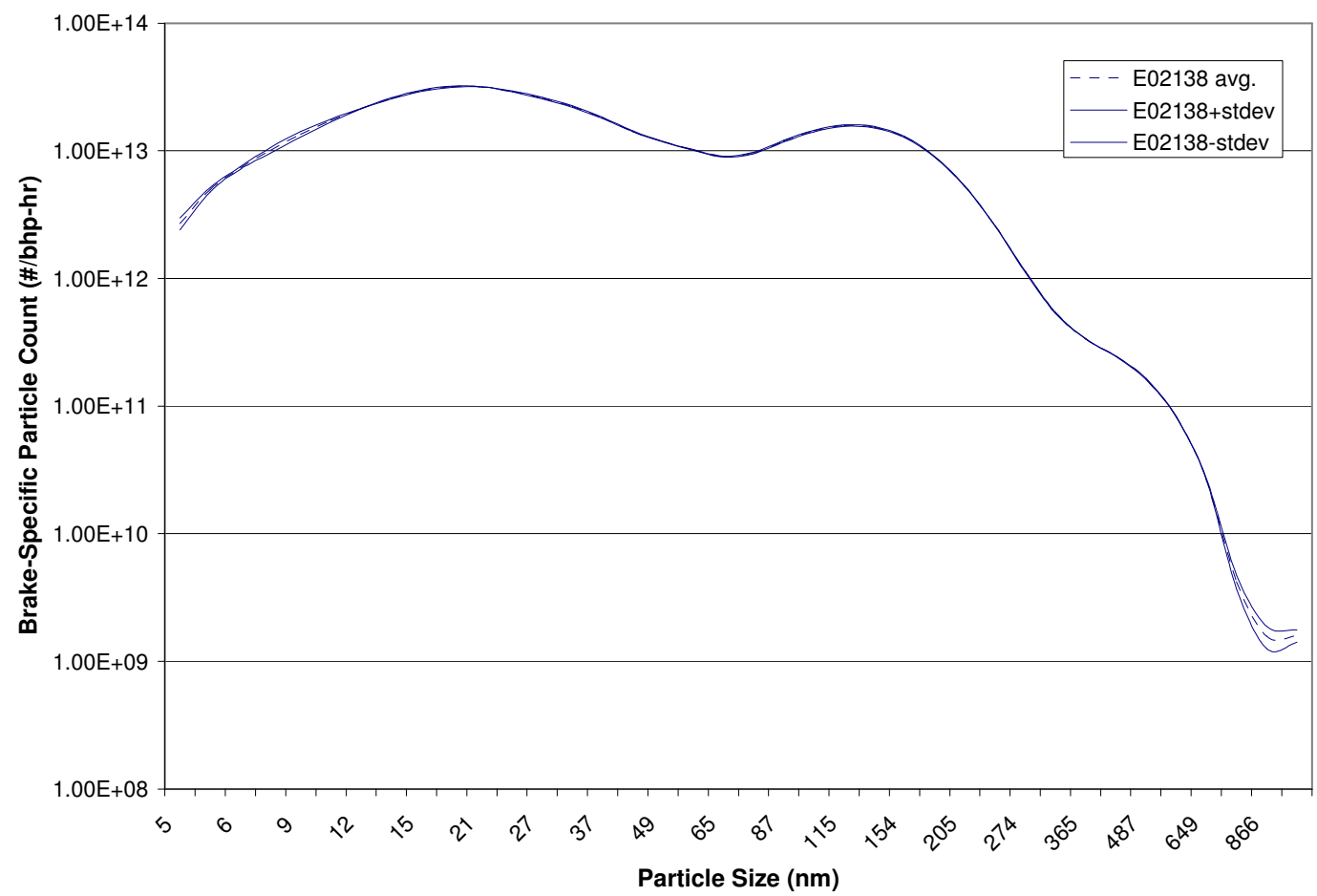

Figure A-18 Average Particle Concentrations of Test Sequence E02138 


\section{A.4.6 Worst Repeatability for Rebuilt DDC S60}

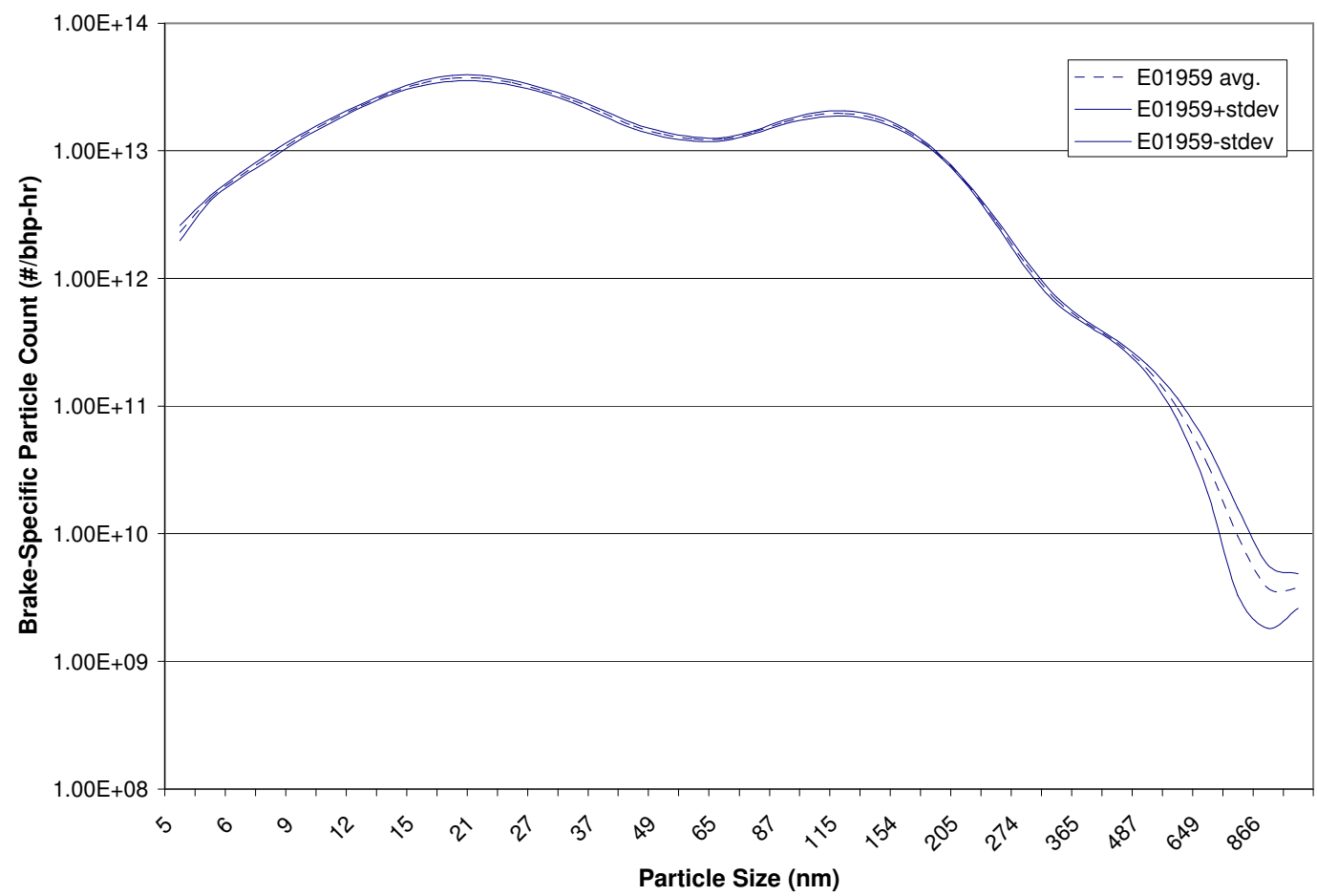

Figure A-19 Average Particle Concentrations of Test Sequence E01959 


\section{A.5 COMPARISON OF TEOM AND DMS DATA}

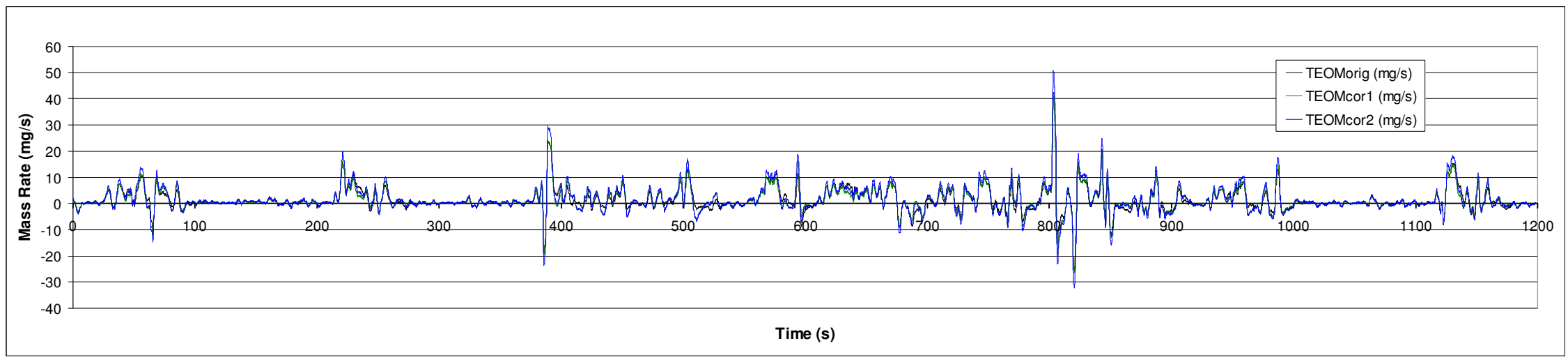

Figure A-20 PM Mass-Rate of E01920_02 as Measured with the TEOM

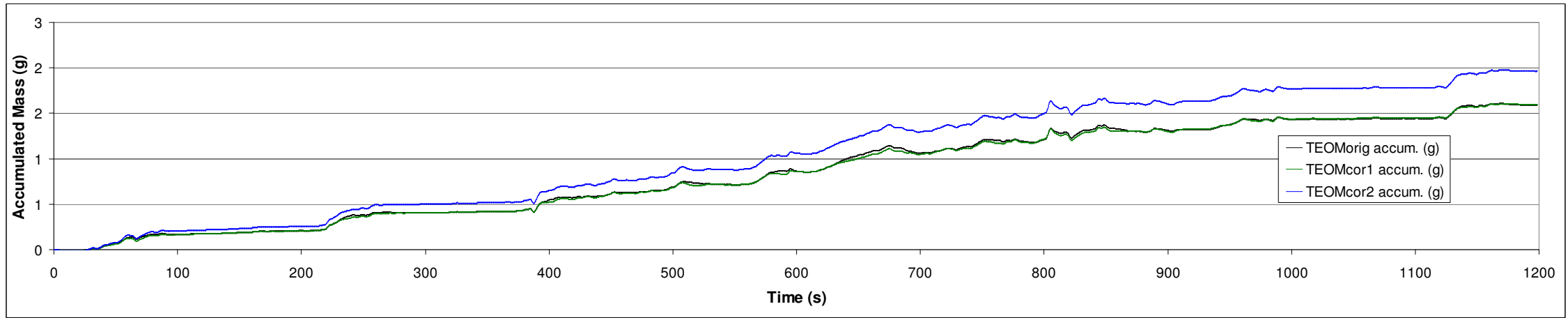

Figure A-21 Corrected and Uncorrected PM Mass Accumulation of E01920_02 as Measured with the TEOM 


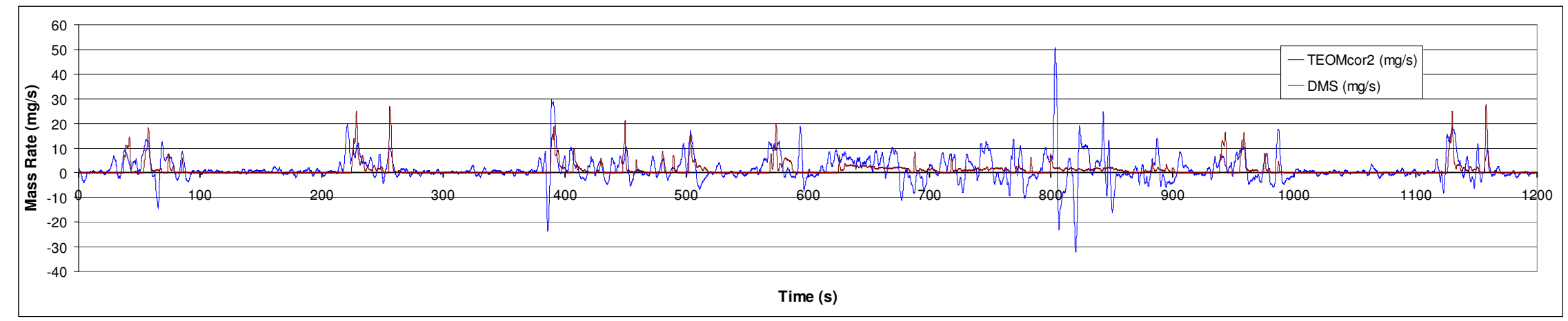

Figure A-22 PM Mass-Rates of E01920_02 as Measured with the TEOM and the DMS

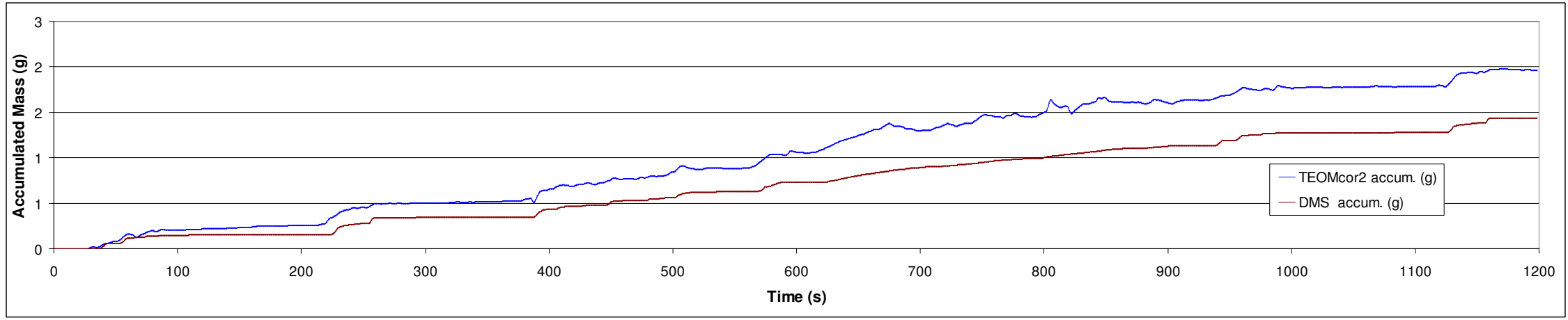

Figure A-23 PM Mass Accumulation of E01920_02 as Measured with the TEOM and the DMS 


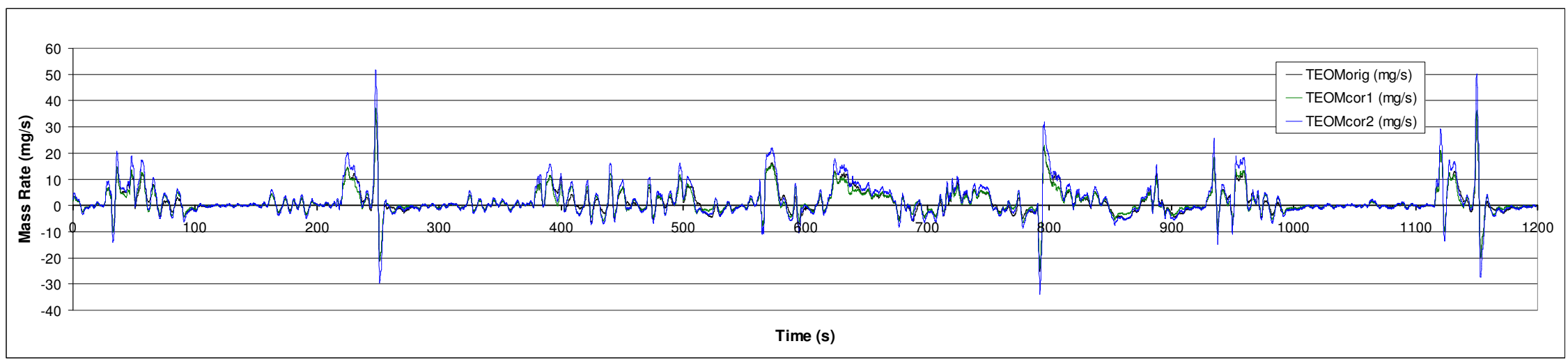

Figure A-24 PM Mass-Rate of E01927_03 as Measured with the TEOM

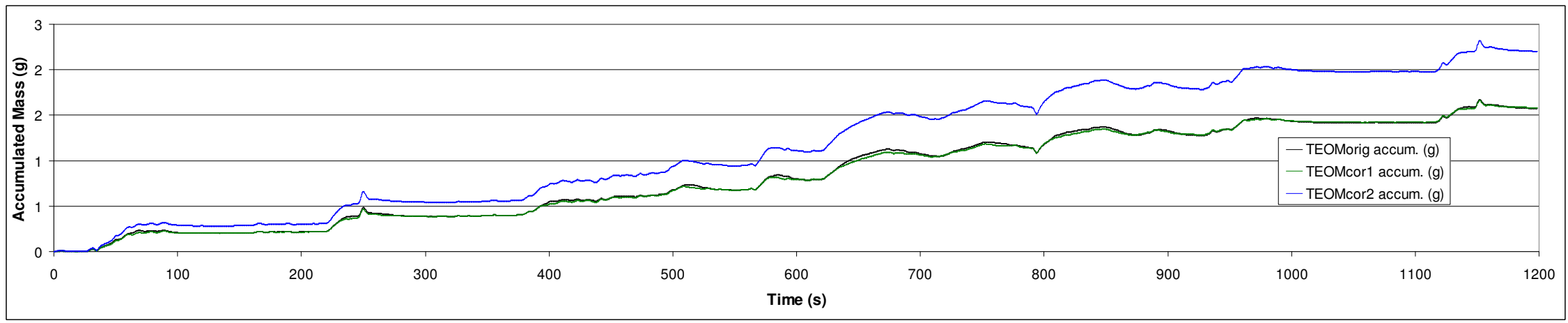

Figure A-25 Corrected and Uncorrected PM Mass Accumulation of E01927_03 as Measured with the TEOM 


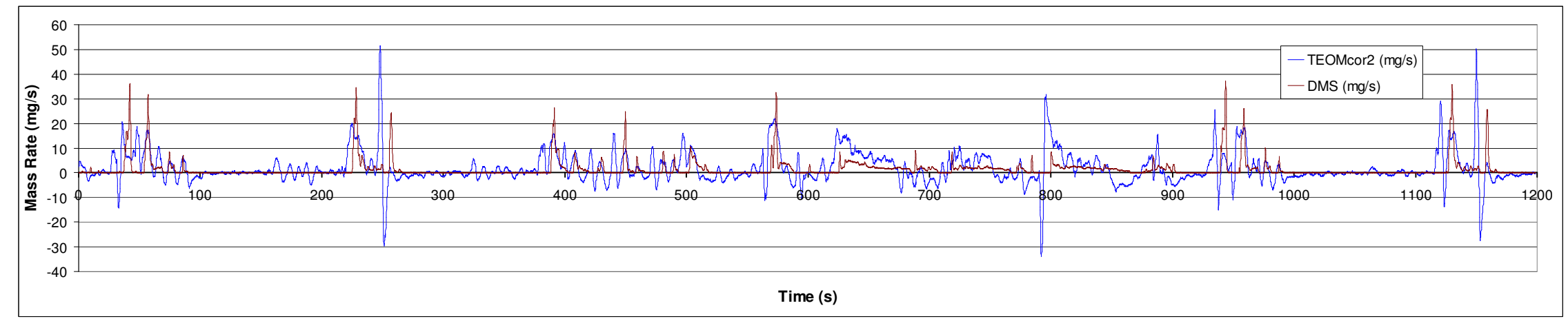

Figure A-26 PM Mass-Rates of E01927_03 as Measured with the TEOM and the DMS

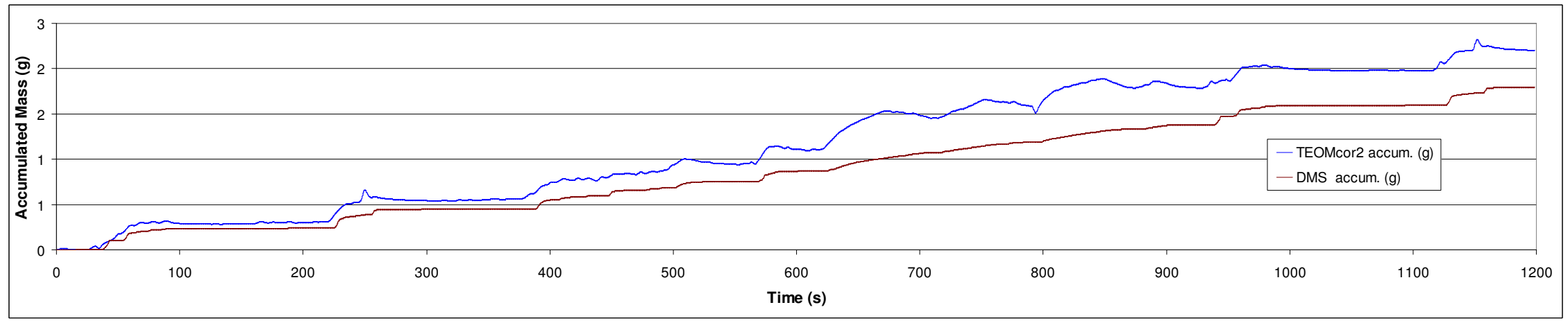

Figure A-27 PM Mass Accumulation of E01927_03 as Measured with the TEOM and the DMS 


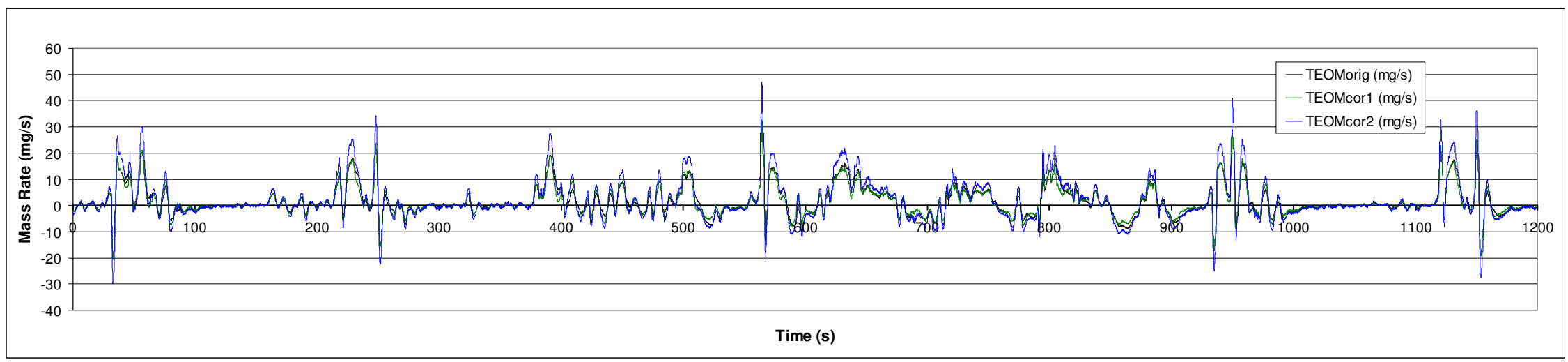

Figure A-28 PM Mass-Rate of E01929_04 as Measured with the TEOM

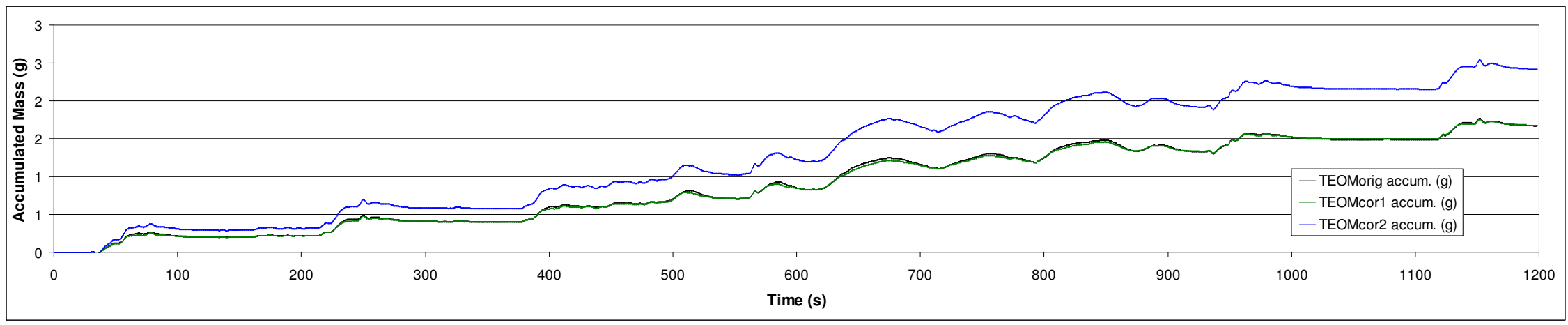

Figure A-29 Corrected and Uncorrected PM Mass Accumulation of E01929_04 as Measured with the TEOM 


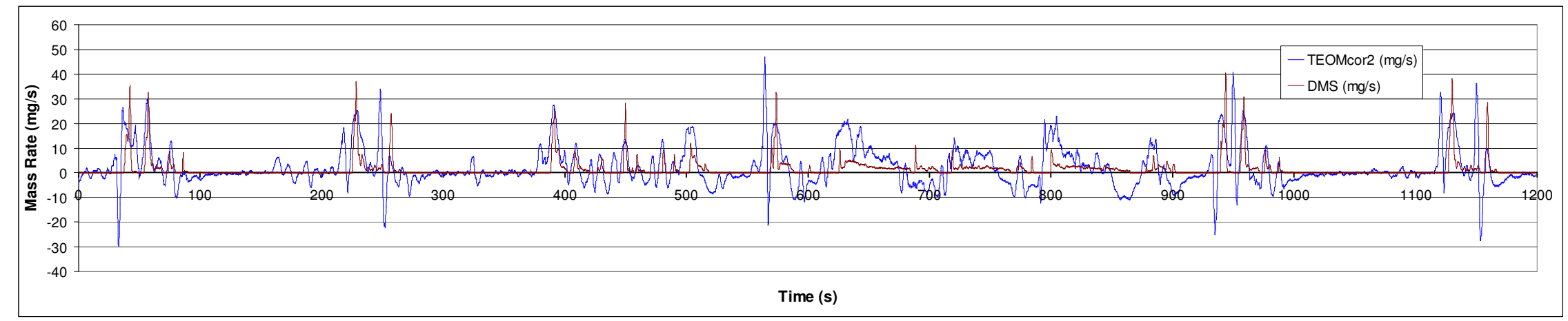

Figure A-30 PM Mass-Rates of E01929_04 as Measured with the TEOM and the DMS

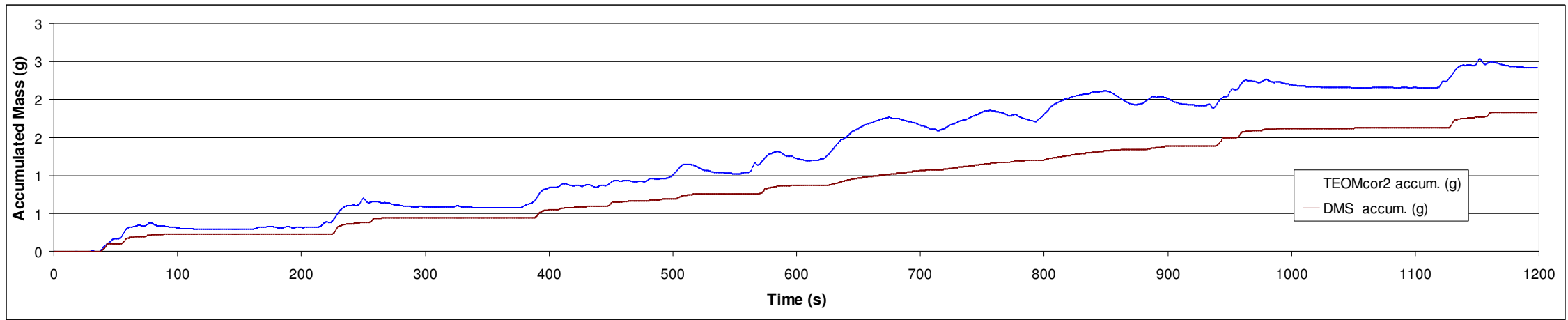

Figure A-31 PM Mass Accumulation of E01929_04 as Measured with the TEOM and the DMS 


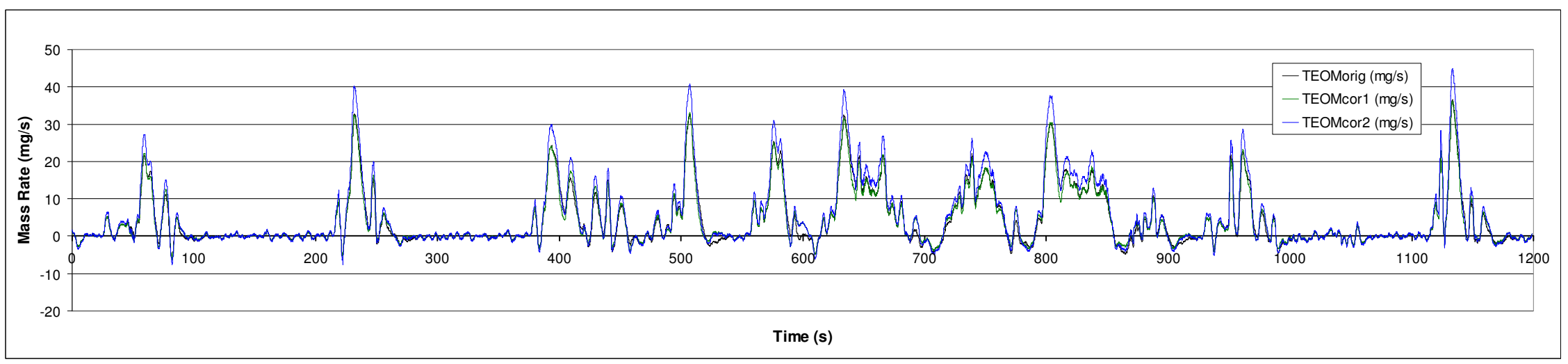

Figure A-32 PM Mass-Rate of E01967_04 as Measured with the TEOM

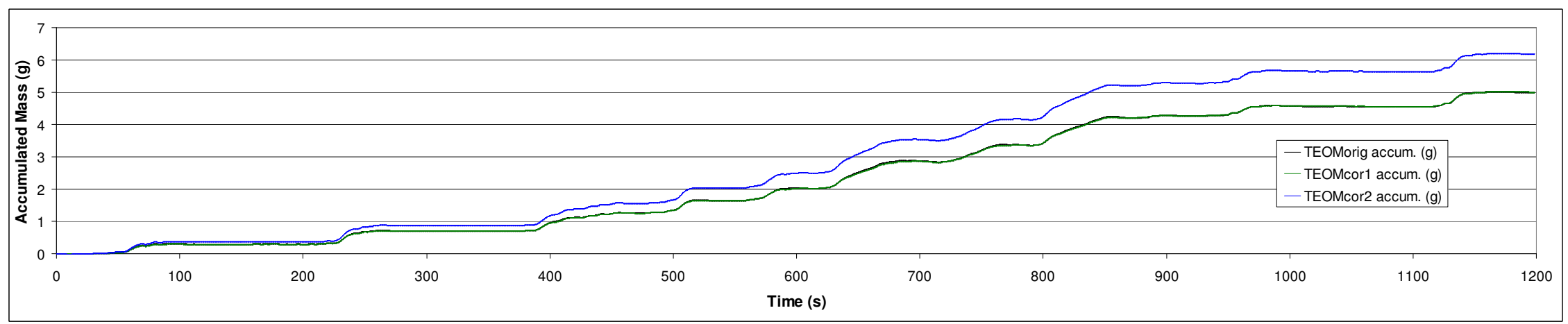

Figure A-33 Corrected and Uncorrected PM Mass Accumulation of E01967_04 as Measured with the TEOM 


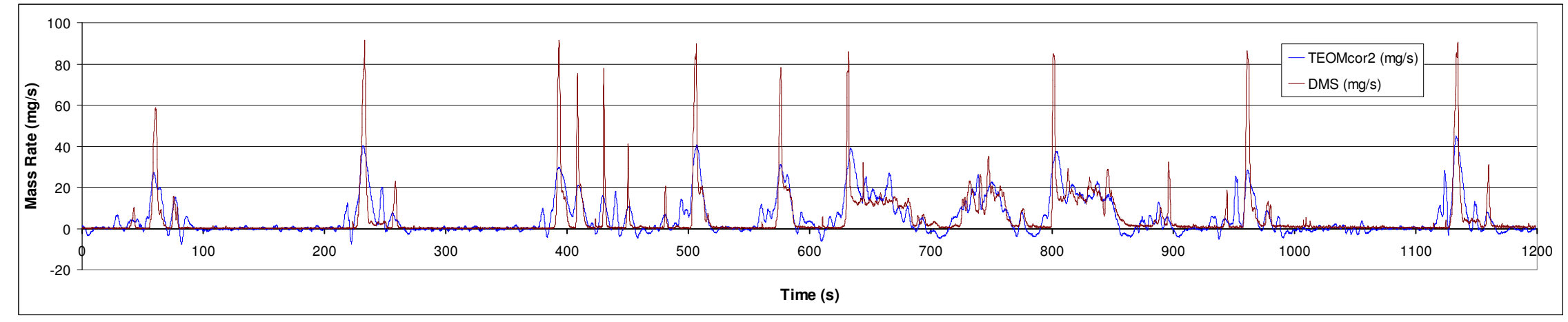

Figure A-34 PM Mass-Rates of E01967_04 as Measured with the TEOM and the DMS

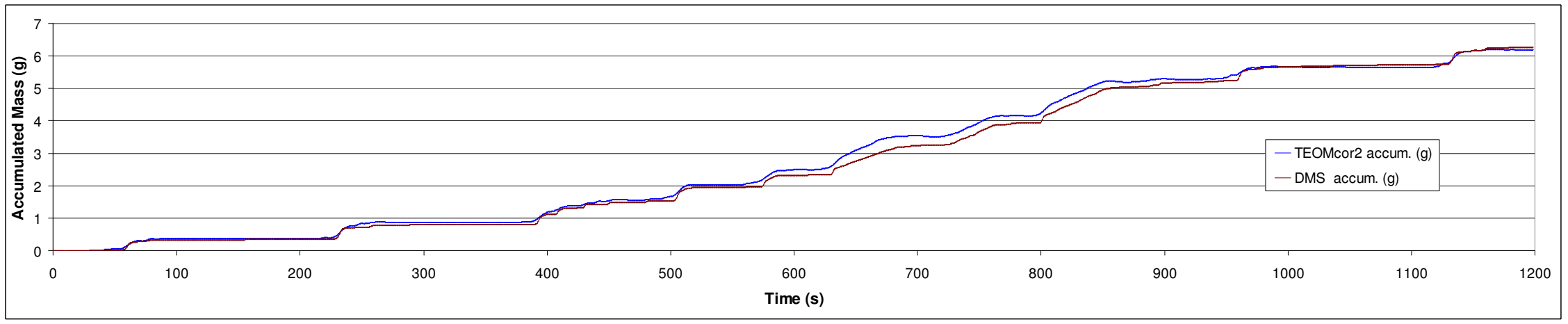

Figure A-35 PM Mass Accumulation of E01967_04 as Measured with the TEOM and the DMS 


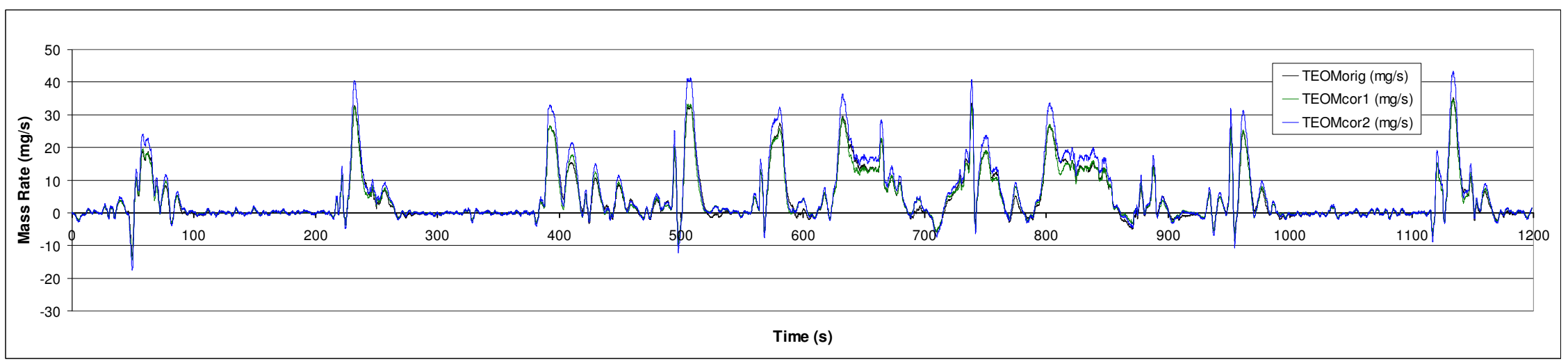

Figure A-36 PM Mass-Rate of E01968_04 as Measured with the TEOM

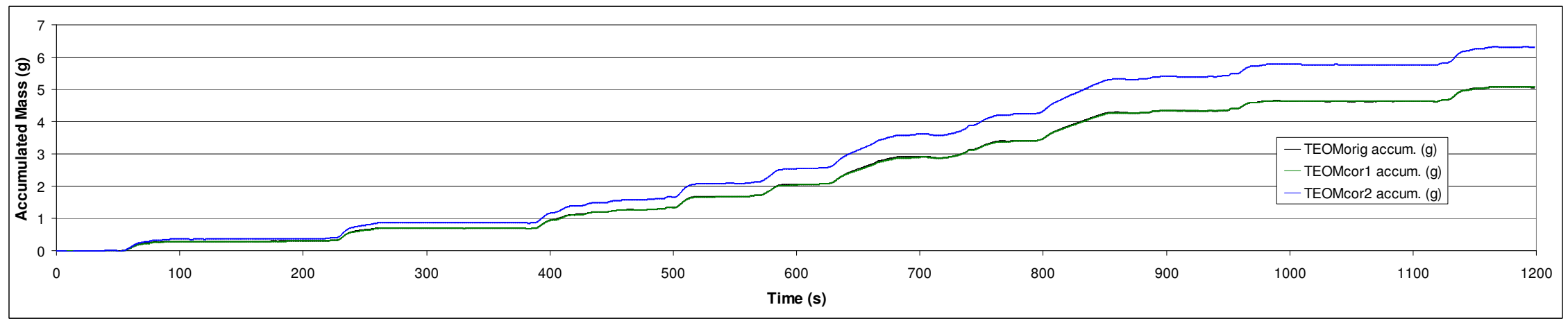

Figure A-37 Corrected and Uncorrected PM Mass Accumulation of E01968_04 as Measured with the TEOM 


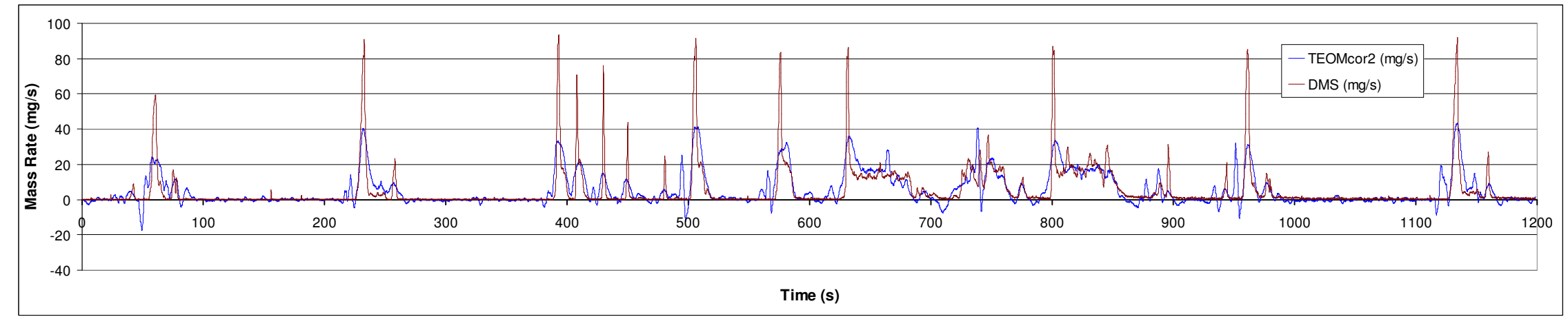

Figure A-38 PM Mass-Rates of E01968_04 as Measured with the TEOM and the DMS

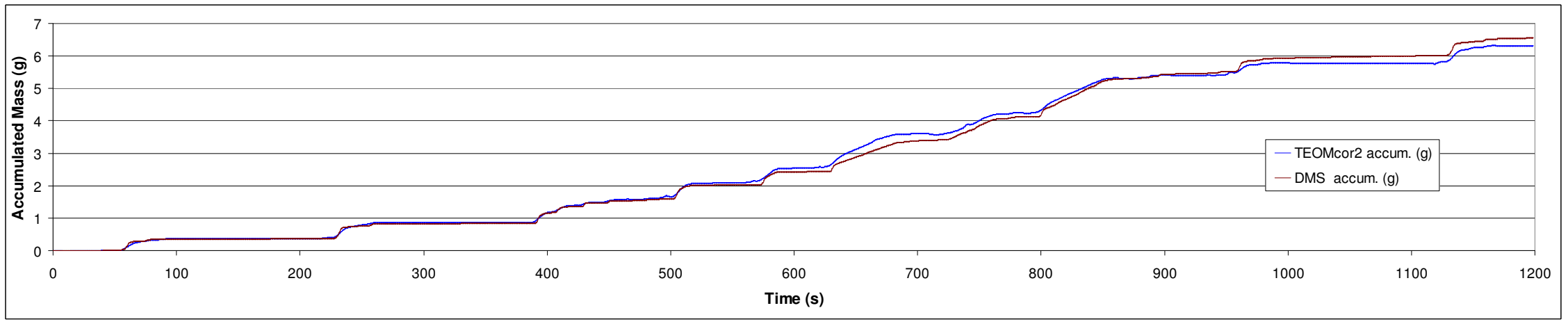

Figure A-39 PM Mass Accumulation of E01968_04 as Measured with the TEOM and the DMS 


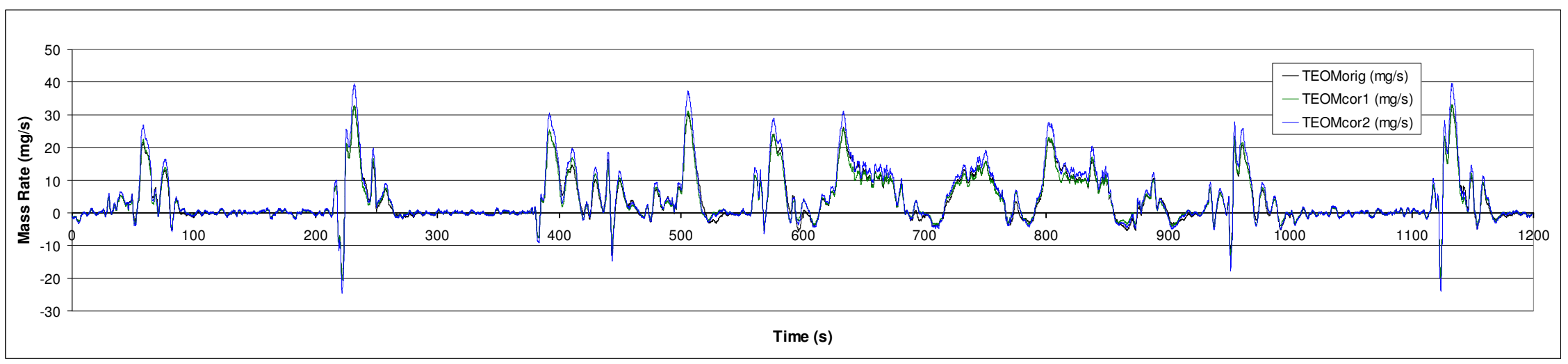

Figure A-40 PM Mass-Rate of E01976_04 as Measured with the TEOM

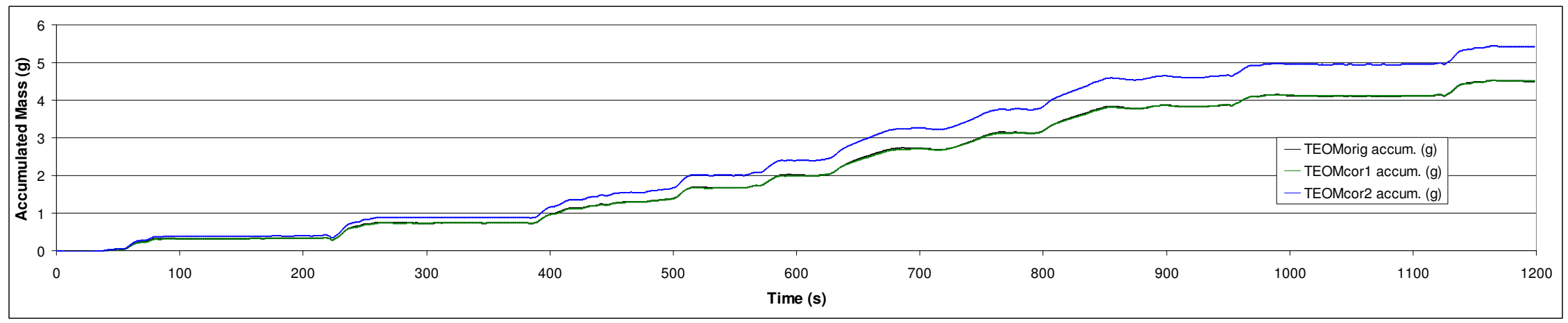

Figure A-41 Corrected and Uncorrected PM Mass Accumulation of E01976_04 as Measured with the TEOM 


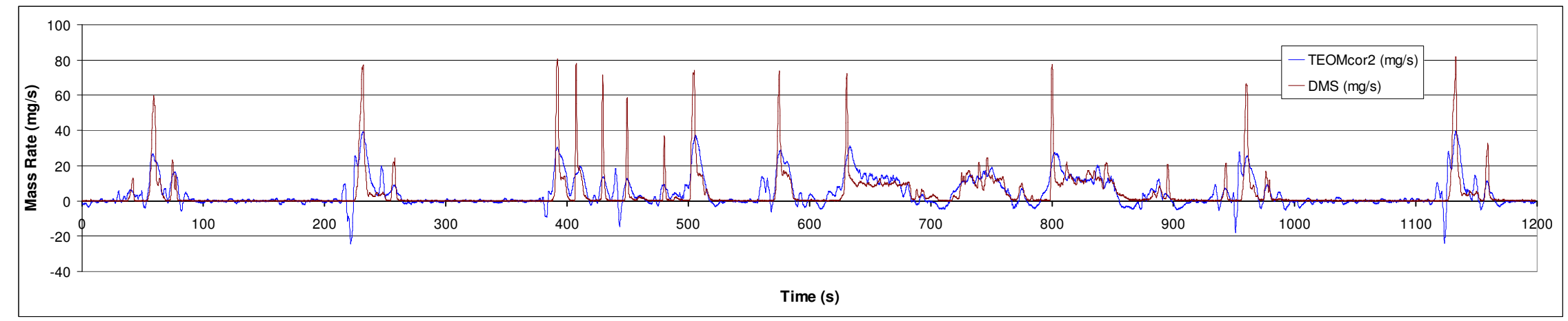

Figure A-42 PM Mass-Rates of E01976_04 as Measured with the TEOM and the DMS

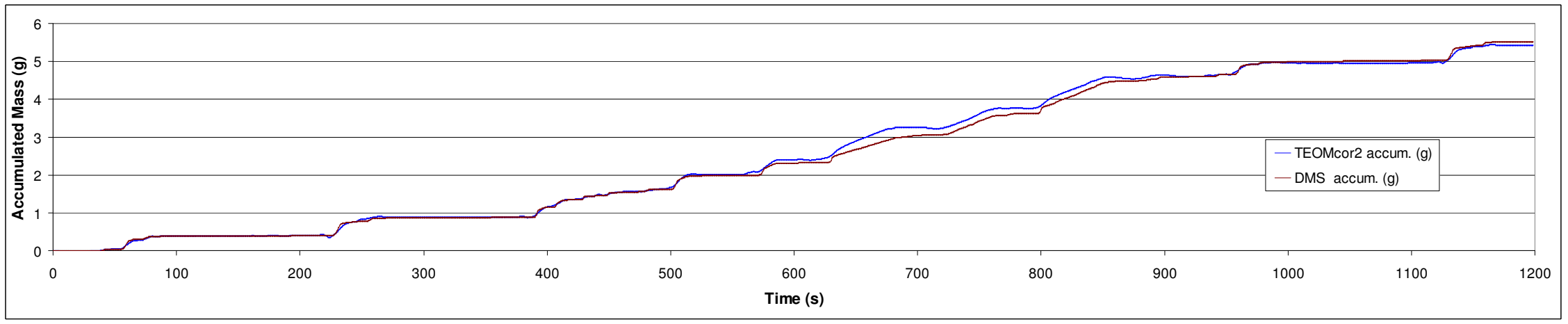

Figure A-43 PM Mass Accumulation of E01976_04 as Measured with the TEOM and the DMS 


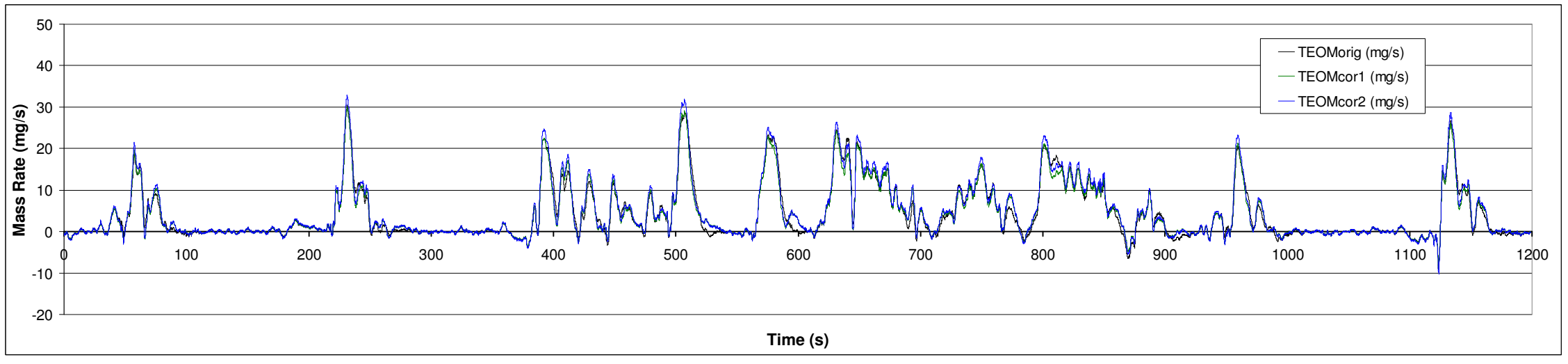

Figure A-44 PM Mass-Rate of E02124_02 as Measured with the TEOM

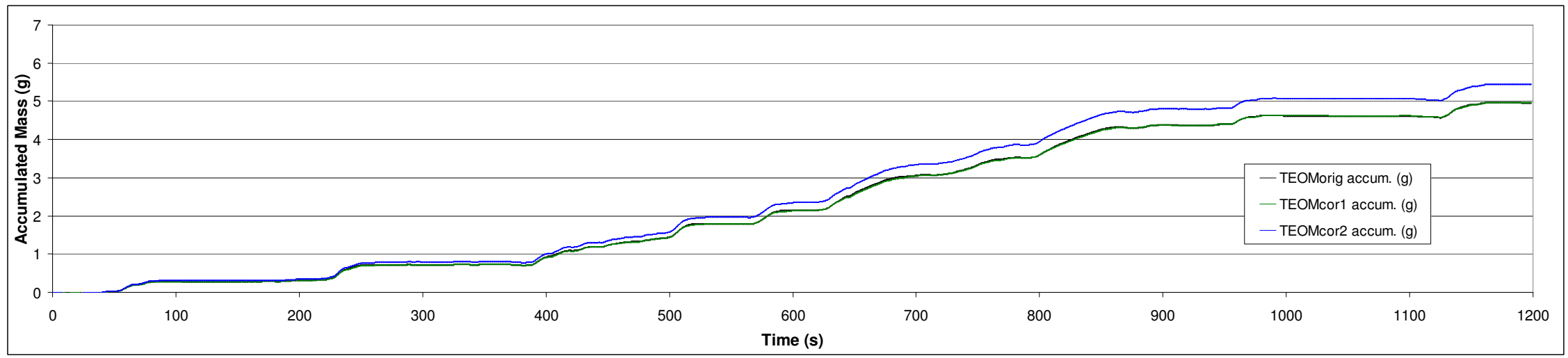

Figure A-45 Corrected and Uncorrected PM Mass Accumulation of E02124_02 as Measured with the TEOM 


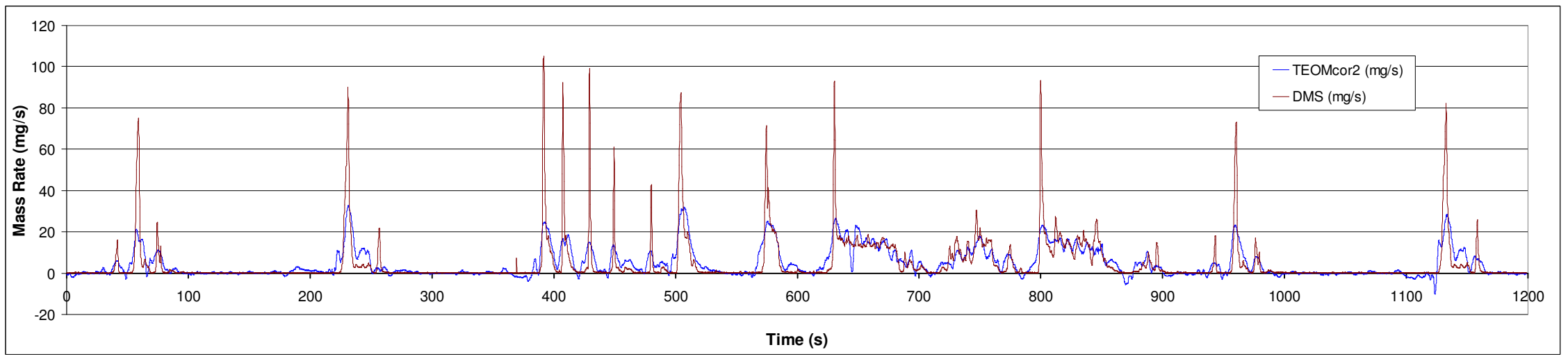

Figure A-46 PM Mass-Rates of E02124_02 as Measured with the TEOM and the DMS

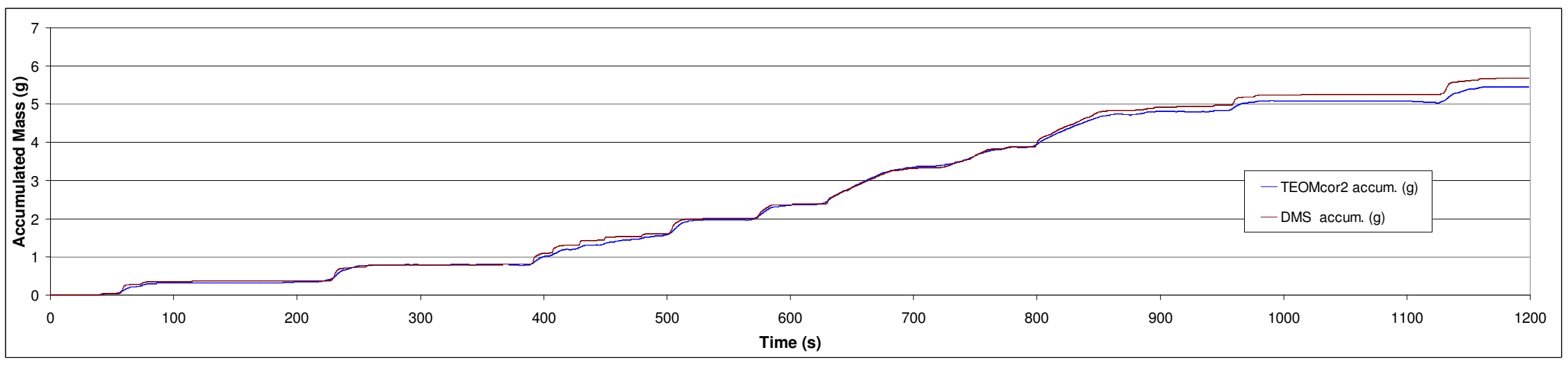

Figure A-47 PM Mass Accumulation of E02124_02 as Measured with the TEOM and the DMS 


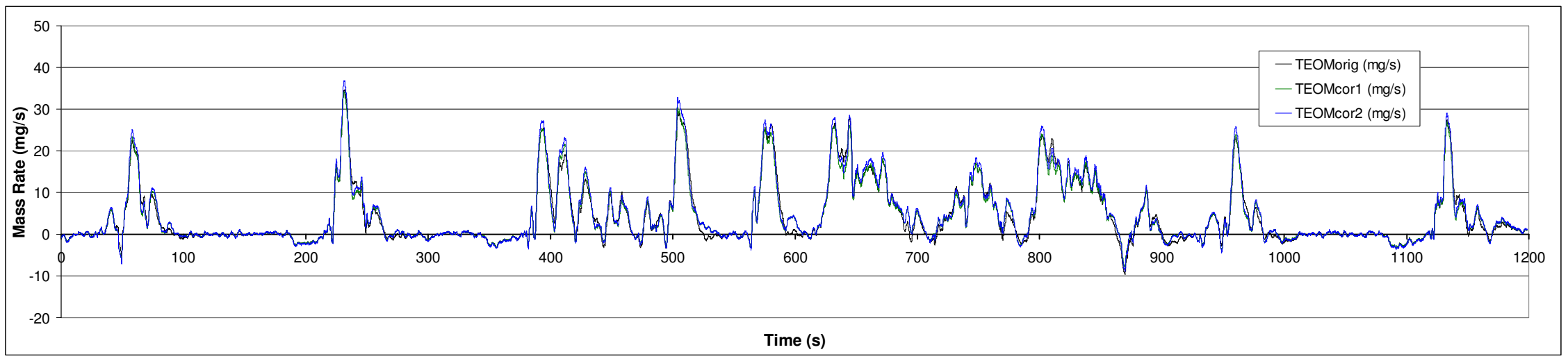

Figure A-48 PM Mass-Rate of E02125_02 as Measured with the TEOM

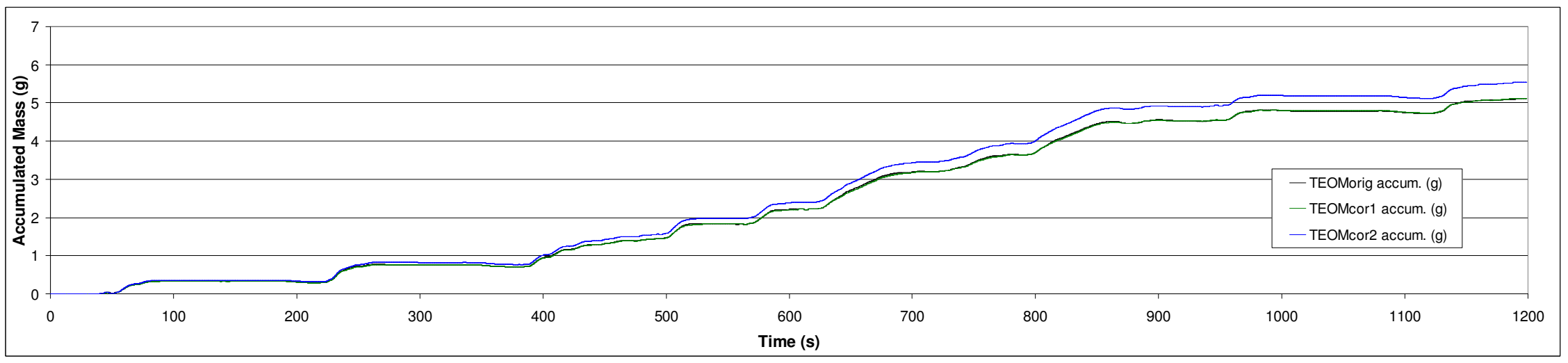

Figure A-49 Corrected and Uncorrected PM Mass Accumulation of E02125_02 as Measured with the TEOM 


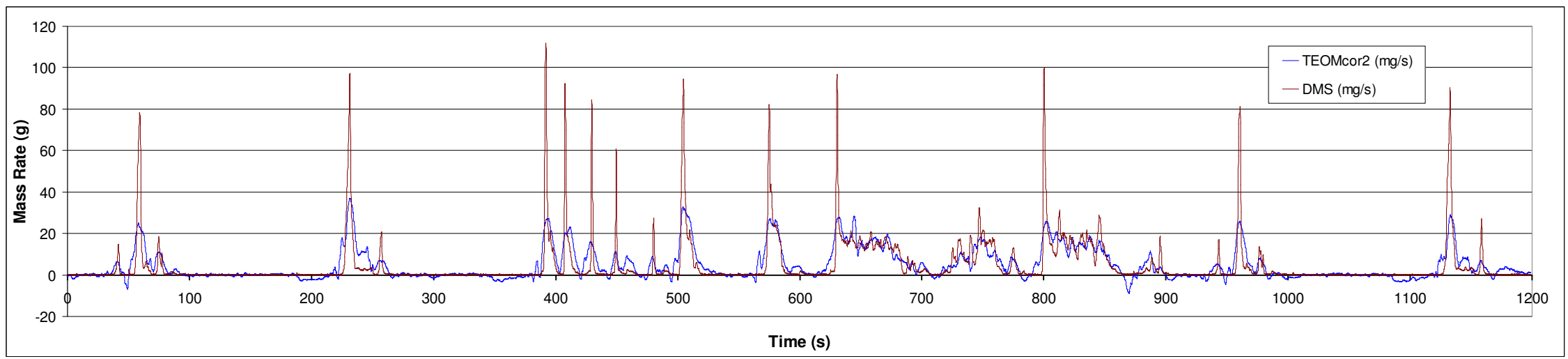

Figure A-50 PM Mass-Rates of E02125_02 as Measured with the TEOM and the DMS

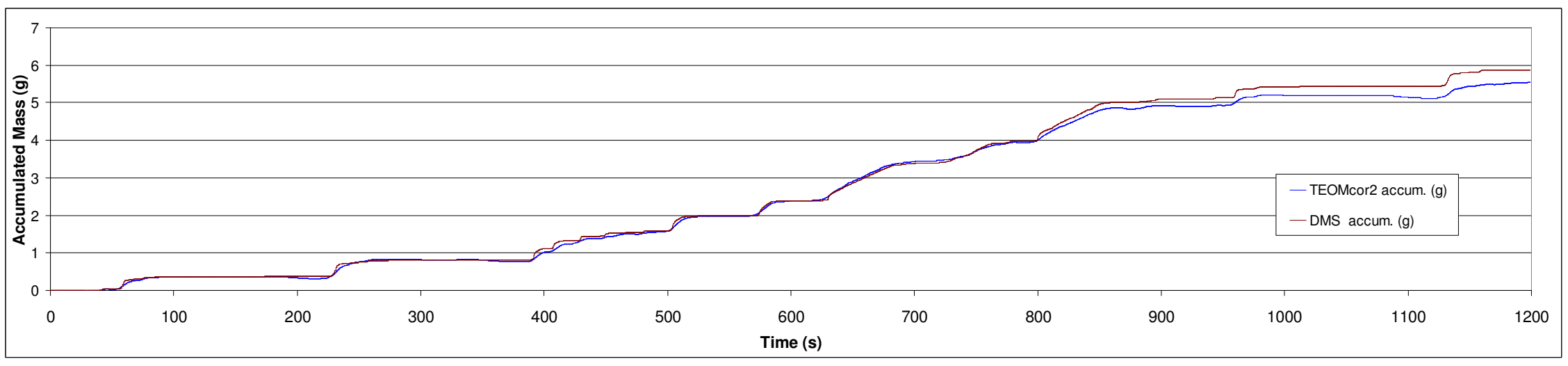

Figure A-51 PM Mass Accumulation of E02125_02 as Measured with the TEOM and the DMS 


\section{A.6 HUMIDITY EFFECTS}

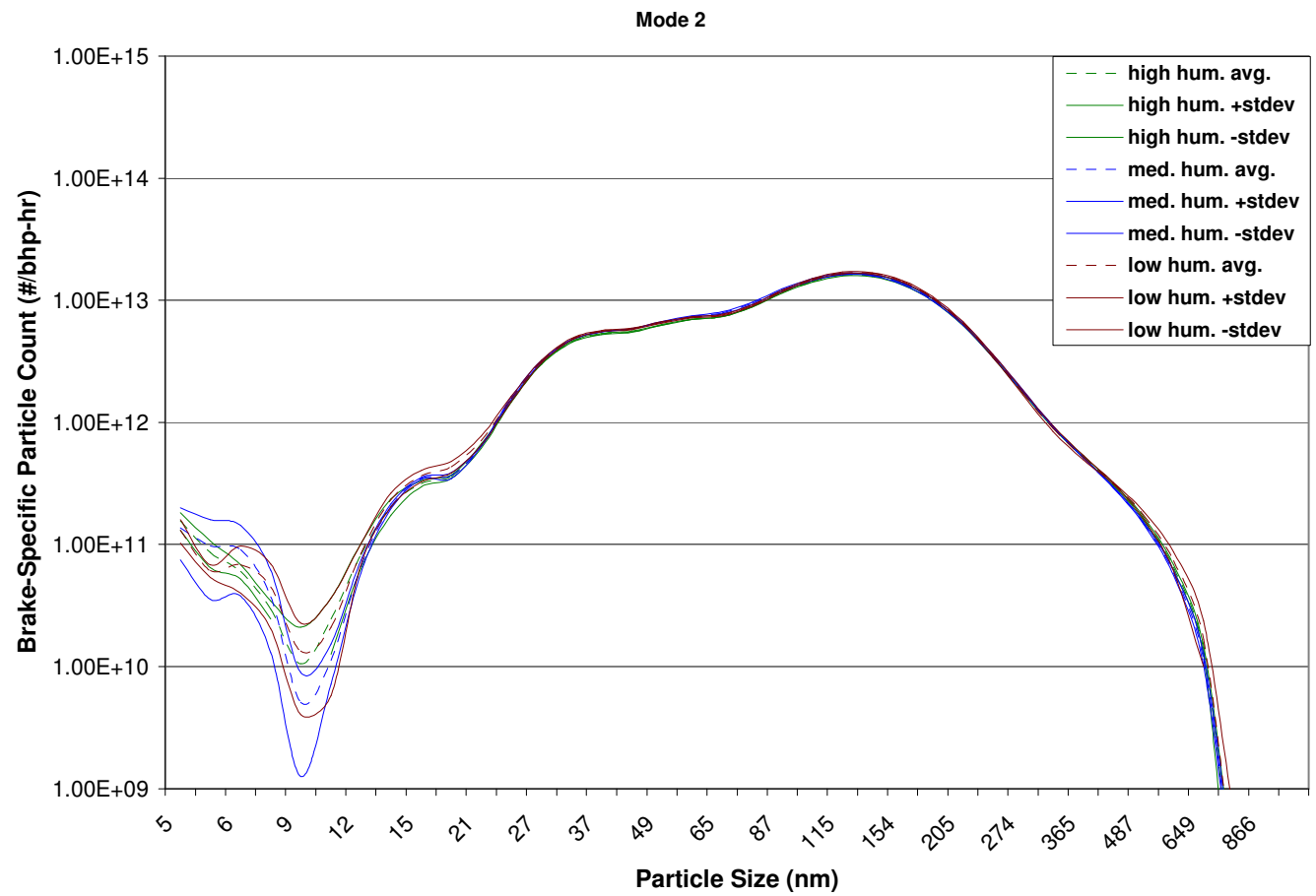

Figure A-52 Particle Concentration Comparison of

High, Medium, and Low Humidity Settings of Mode 2

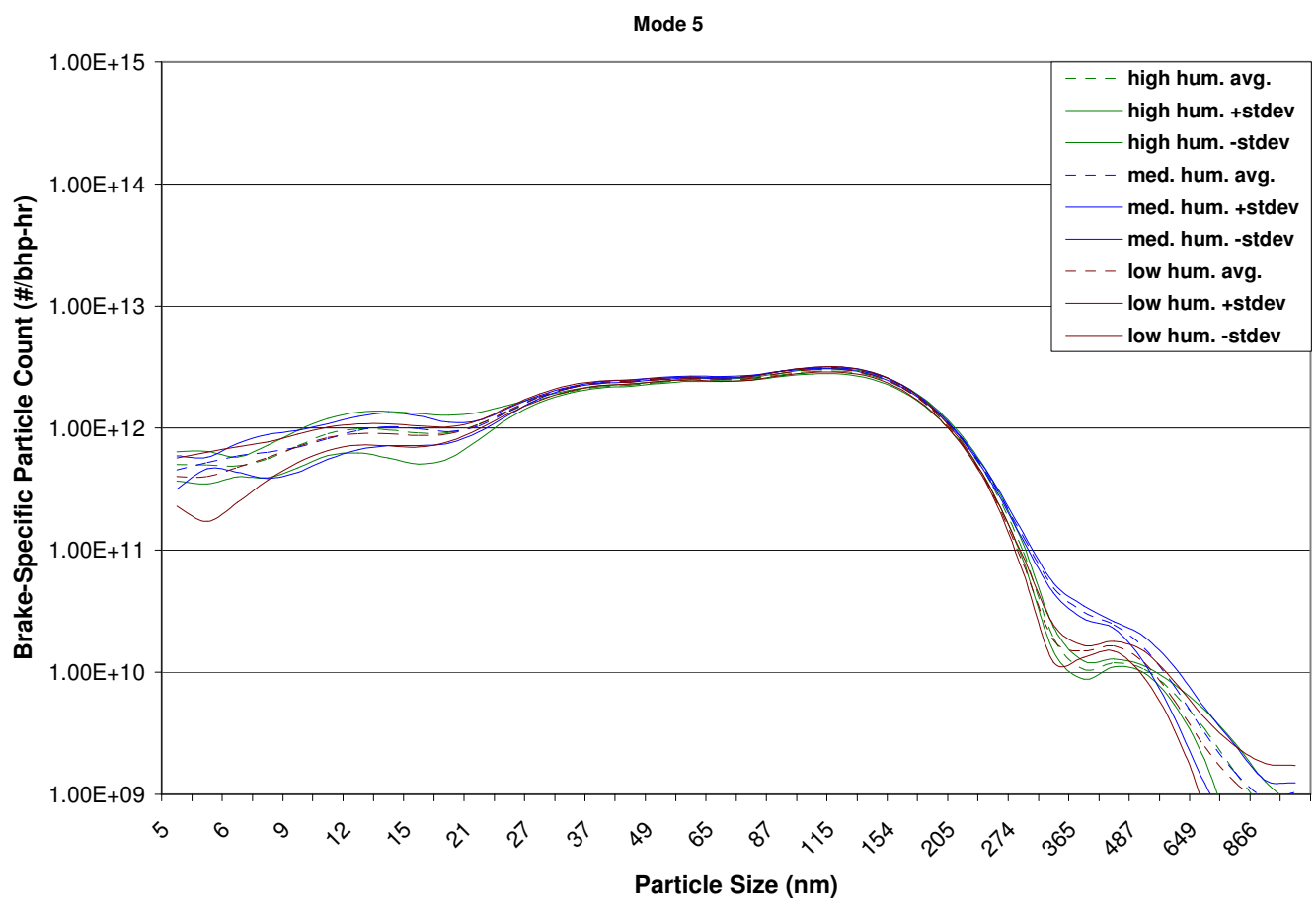

Figure A-53 Particle Concentration Comparison of

High, Medium, and Low Humidity Settings of Mode 5 


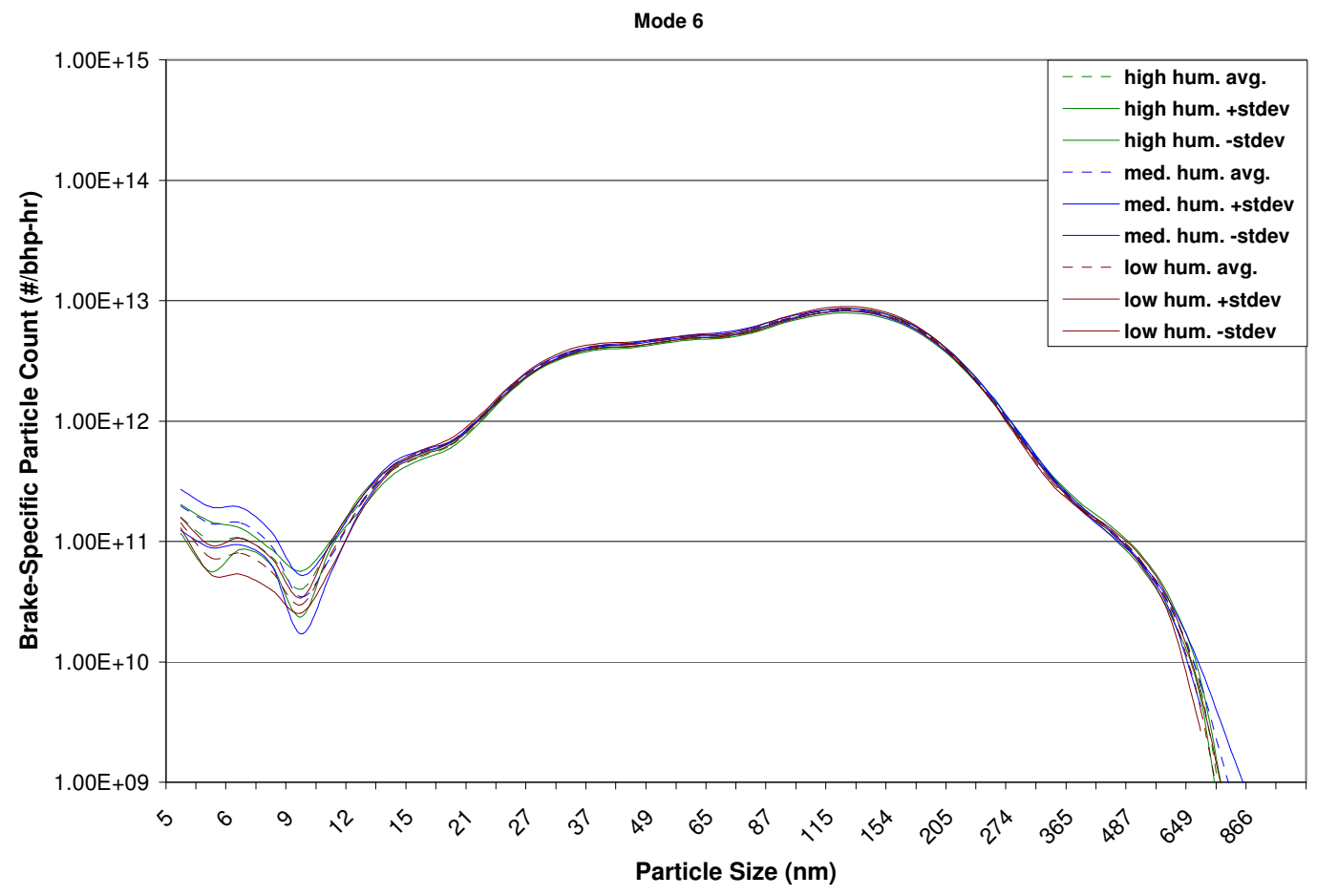

Figure A-54 Particle Concentration Comparison of High, Medium, and Low Humidity Settings of Mode 6

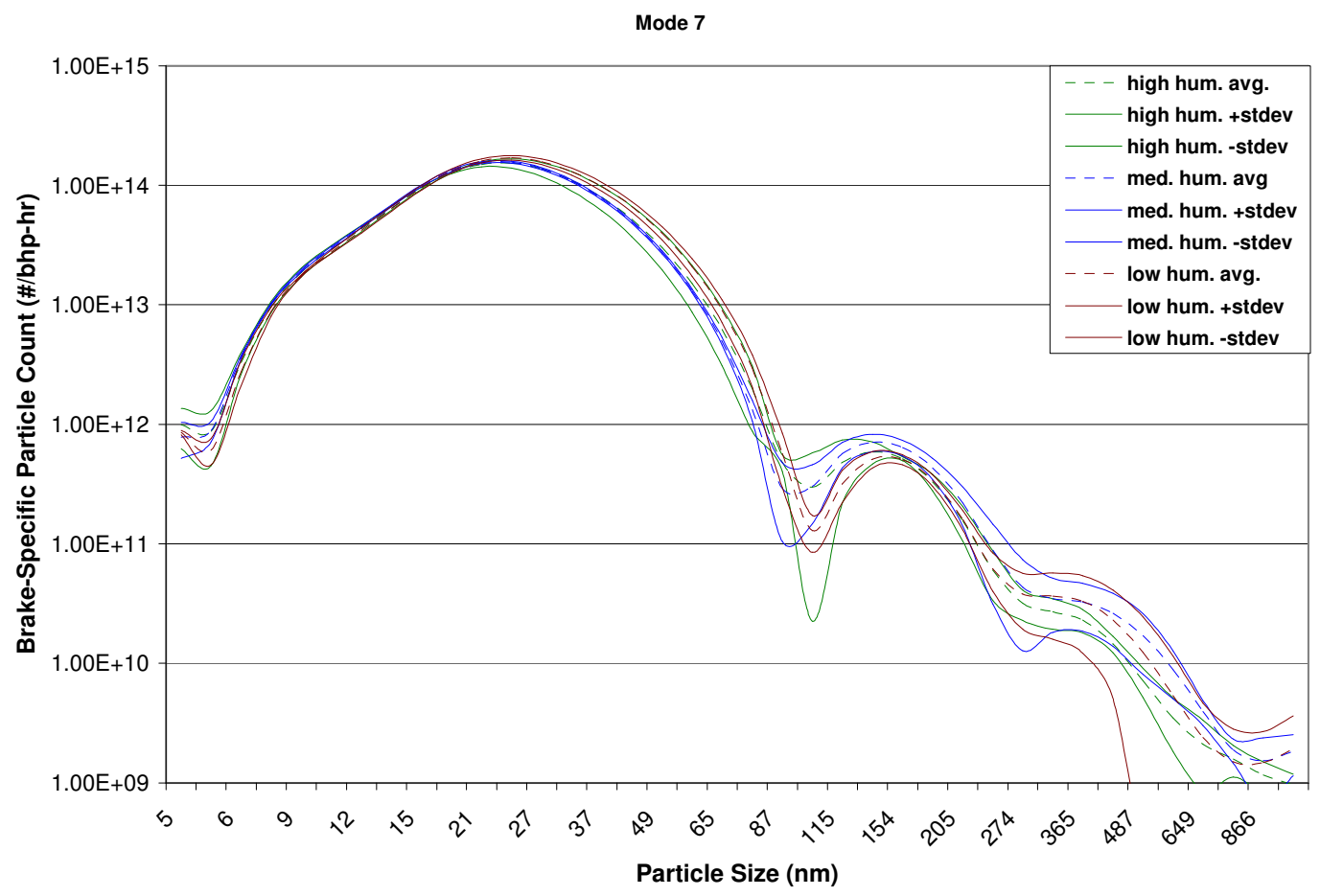

Figure A-55 Particle Concentration Comparison of High, Medium, and Low Humidity Settings of Mode 7 


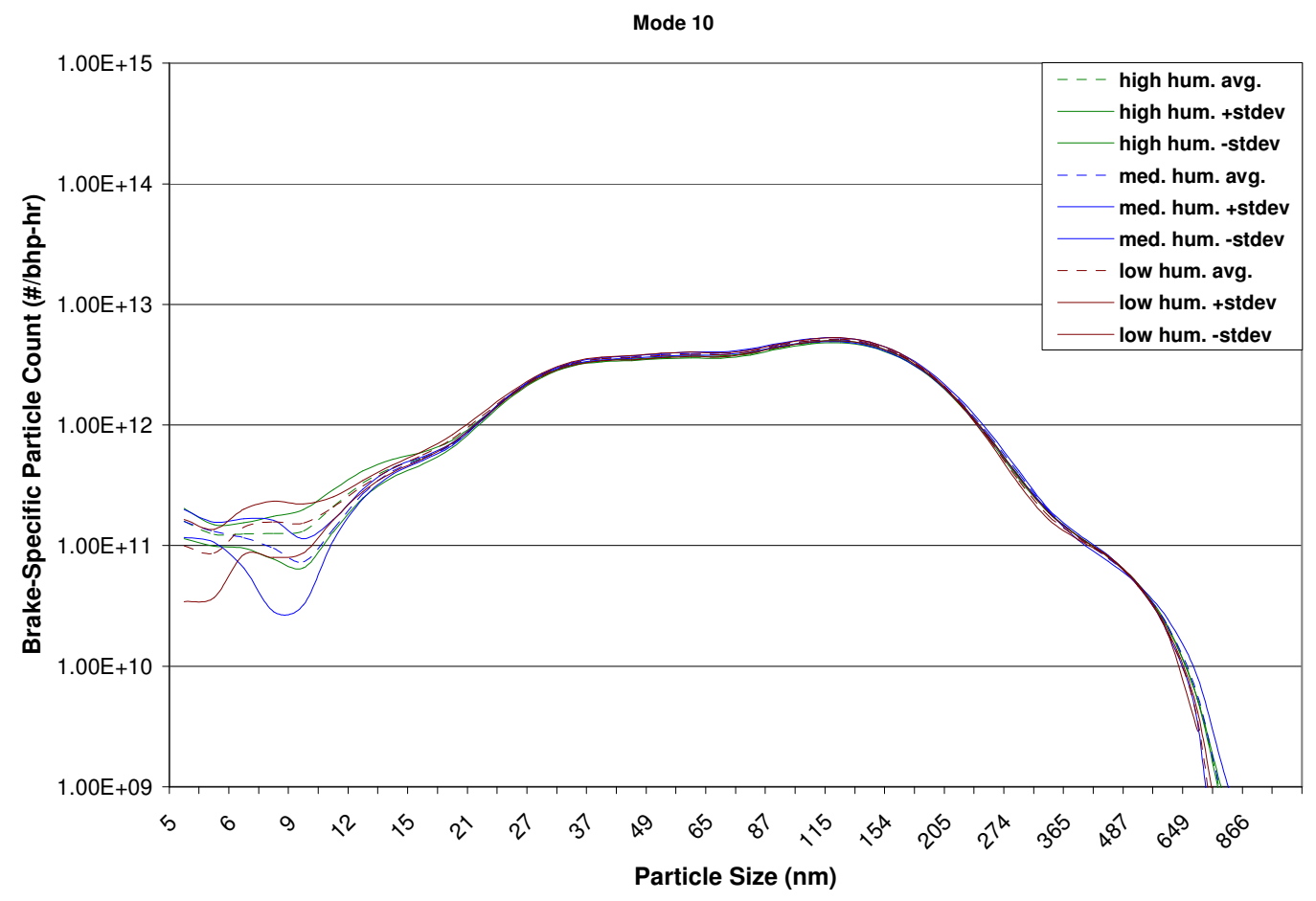

Figure A-56 Particle Concentration Comparison of High, Medium, and Low Humidity Settings of Mode 10

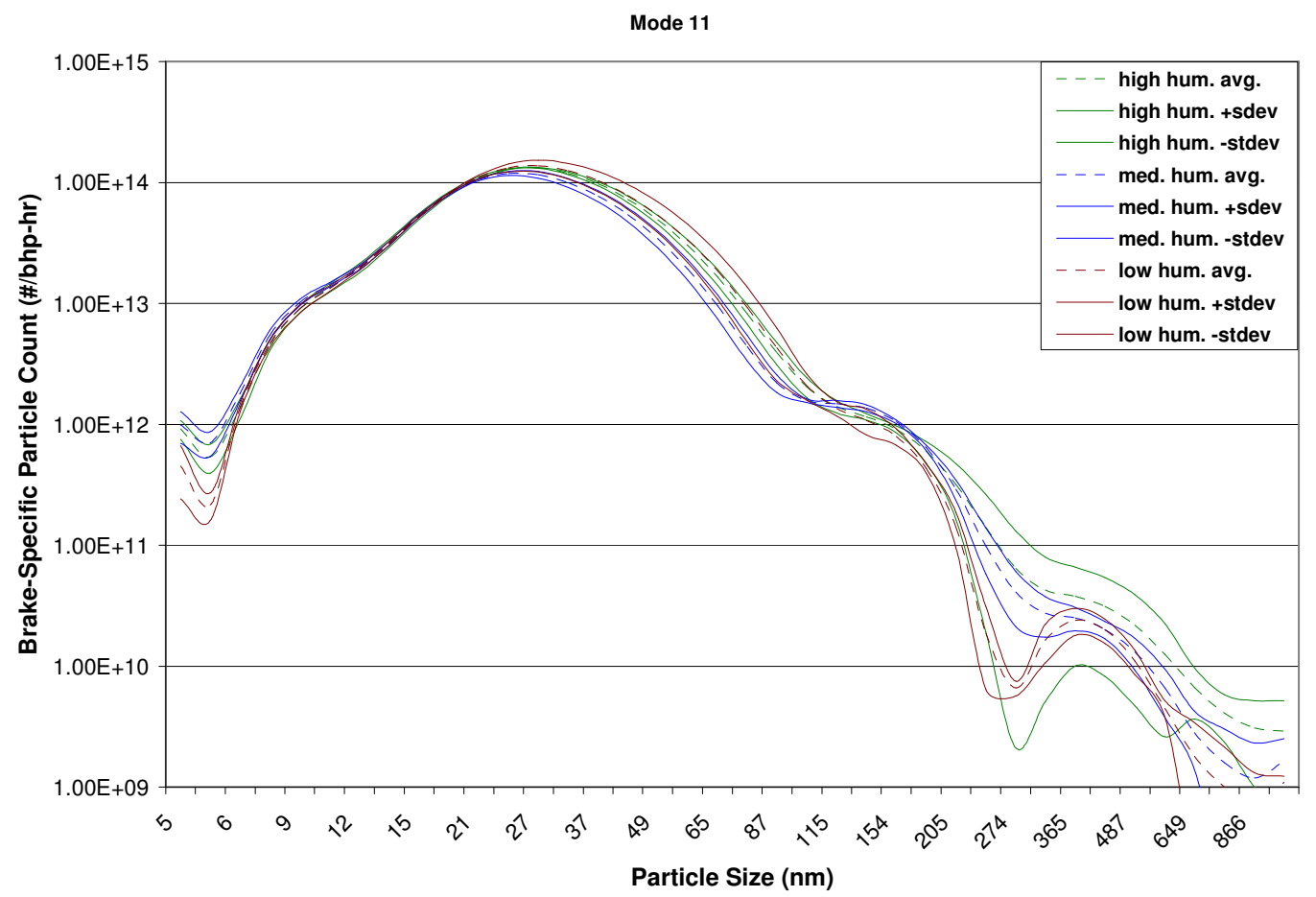

Figure A-57 Particle Concentration Comparison of High, Medium, and Low Humidity Settings of Mode 11 


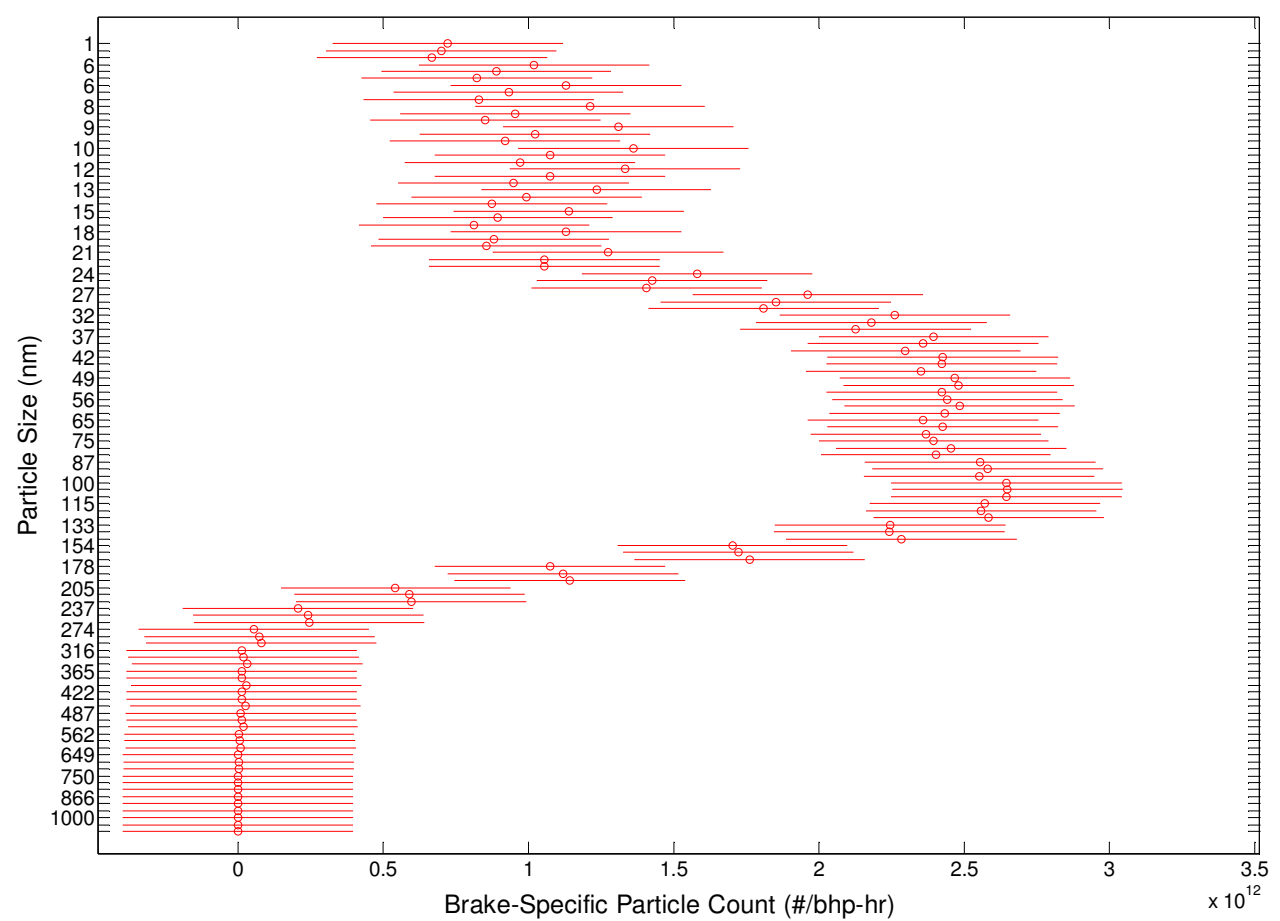

Figure A-58 Particle Sizes that Have Significantly Different Average Values for Mode 3 Due to Humidity Change (According to ANOVA)

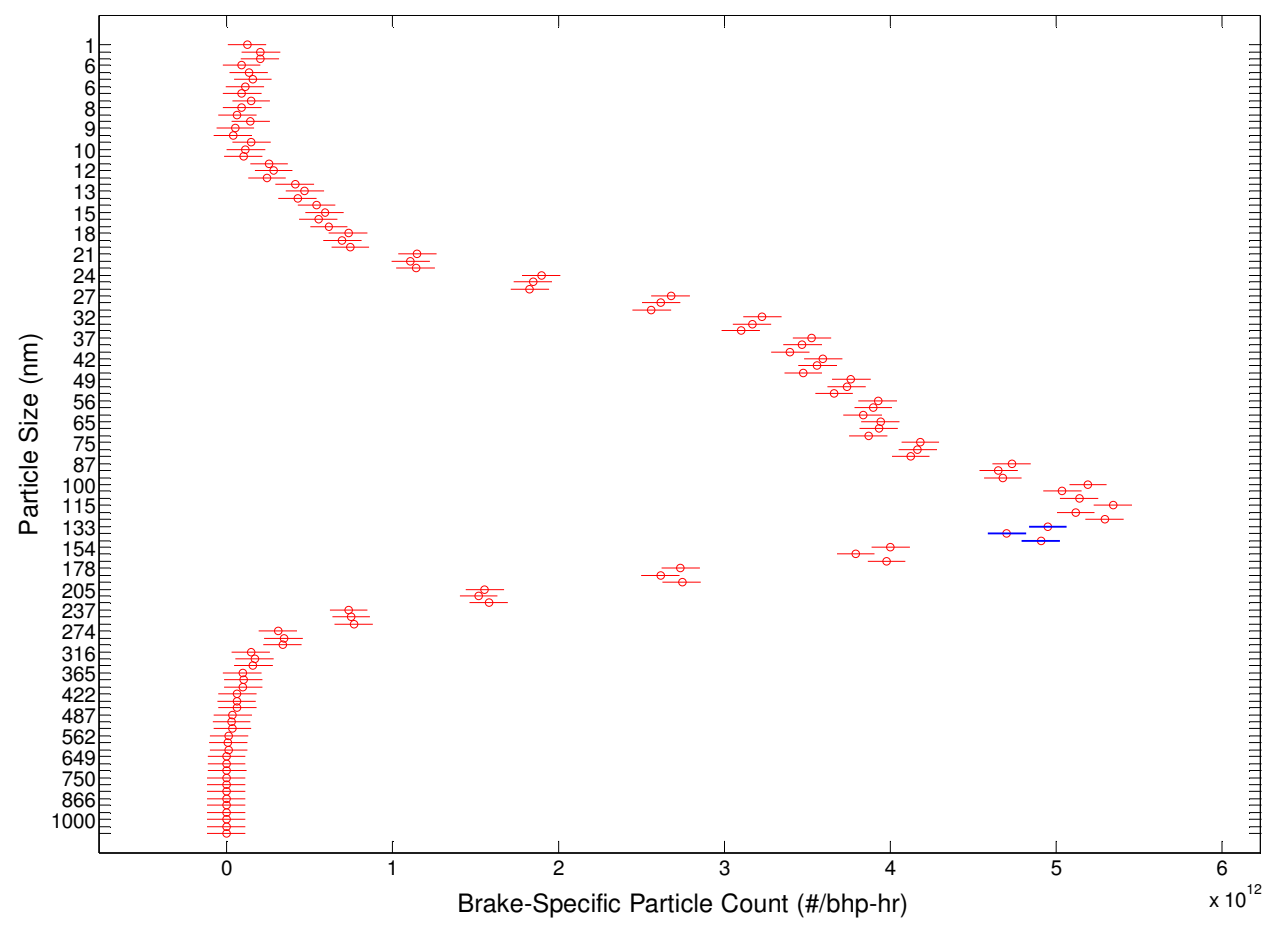

Figure A-59 Particle Sizes that Have Significantly Different Average Values for Mode 4 Due to Humidity Change (According to ANOVA) 


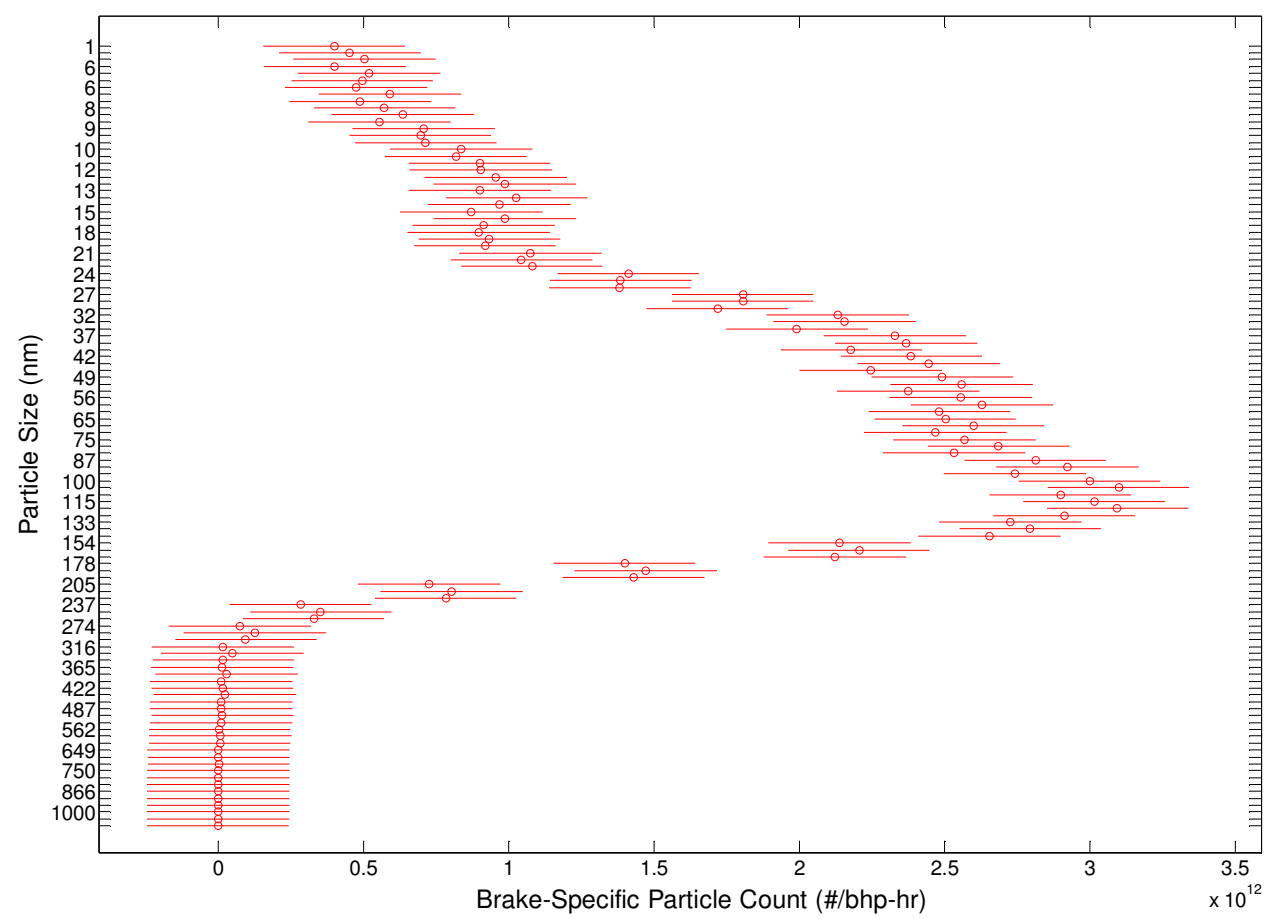

Figure A-60 Particle Sizes that Have Significantly Different Average Values for Mode 5 Due to Humidity Change (According to ANOVA)

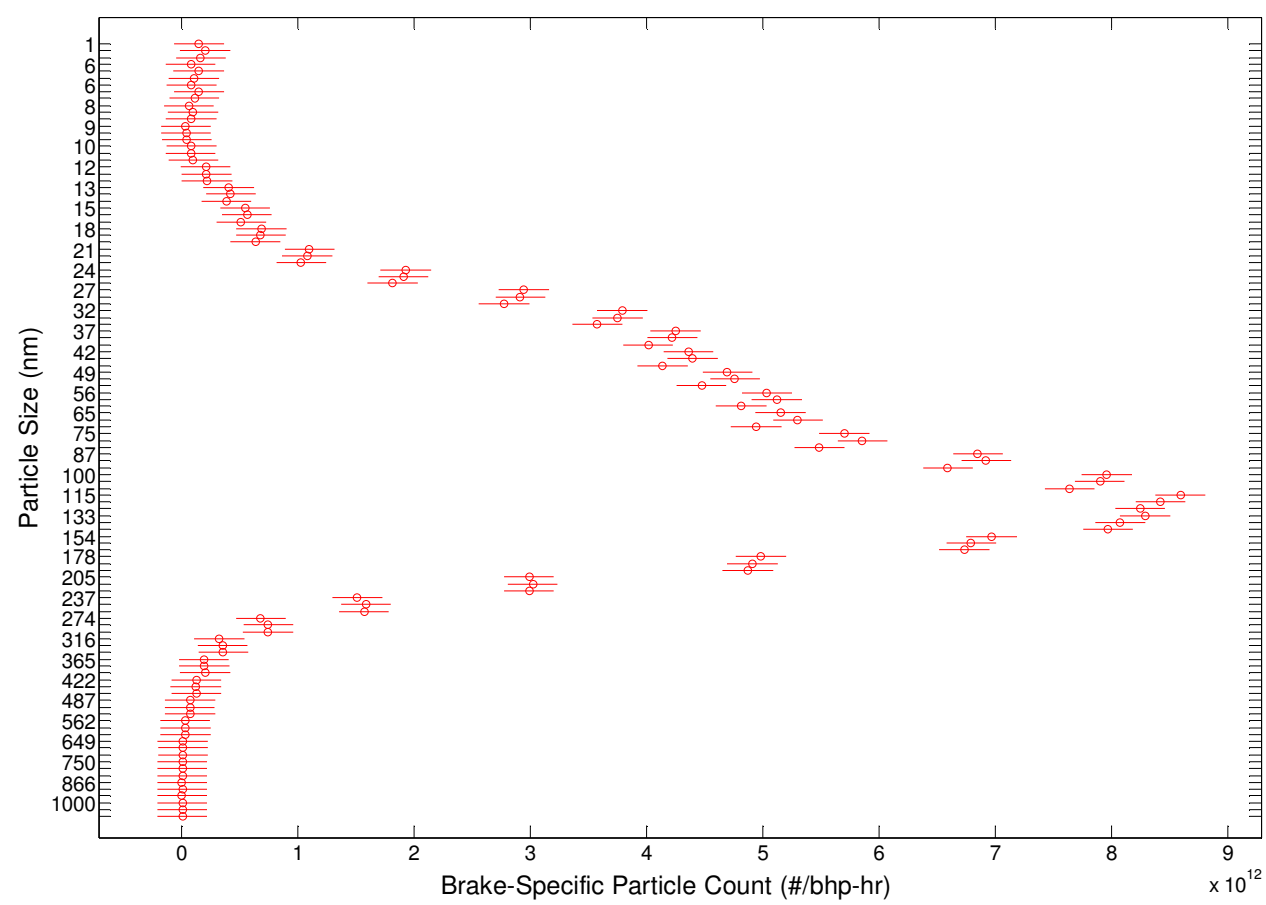

Figure A-61 Particle Sizes that Have Significantly Different Average Values for Mode 6 Due to Humidity Change (According to ANOVA) 


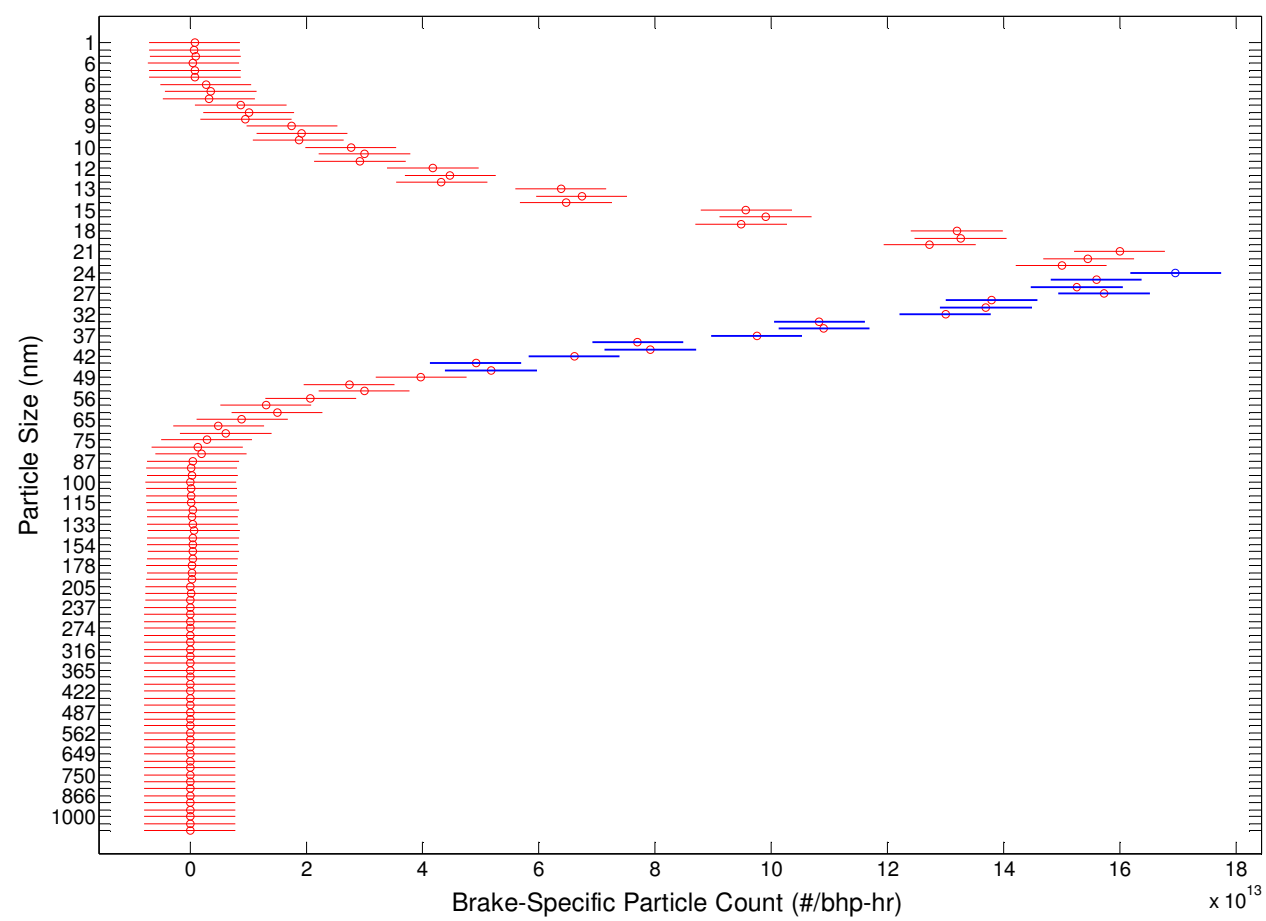

Figure A-62 Particle Sizes that Have Significantly Different Average Values for Mode 7 Due to Humidity Change (According to ANOVA)

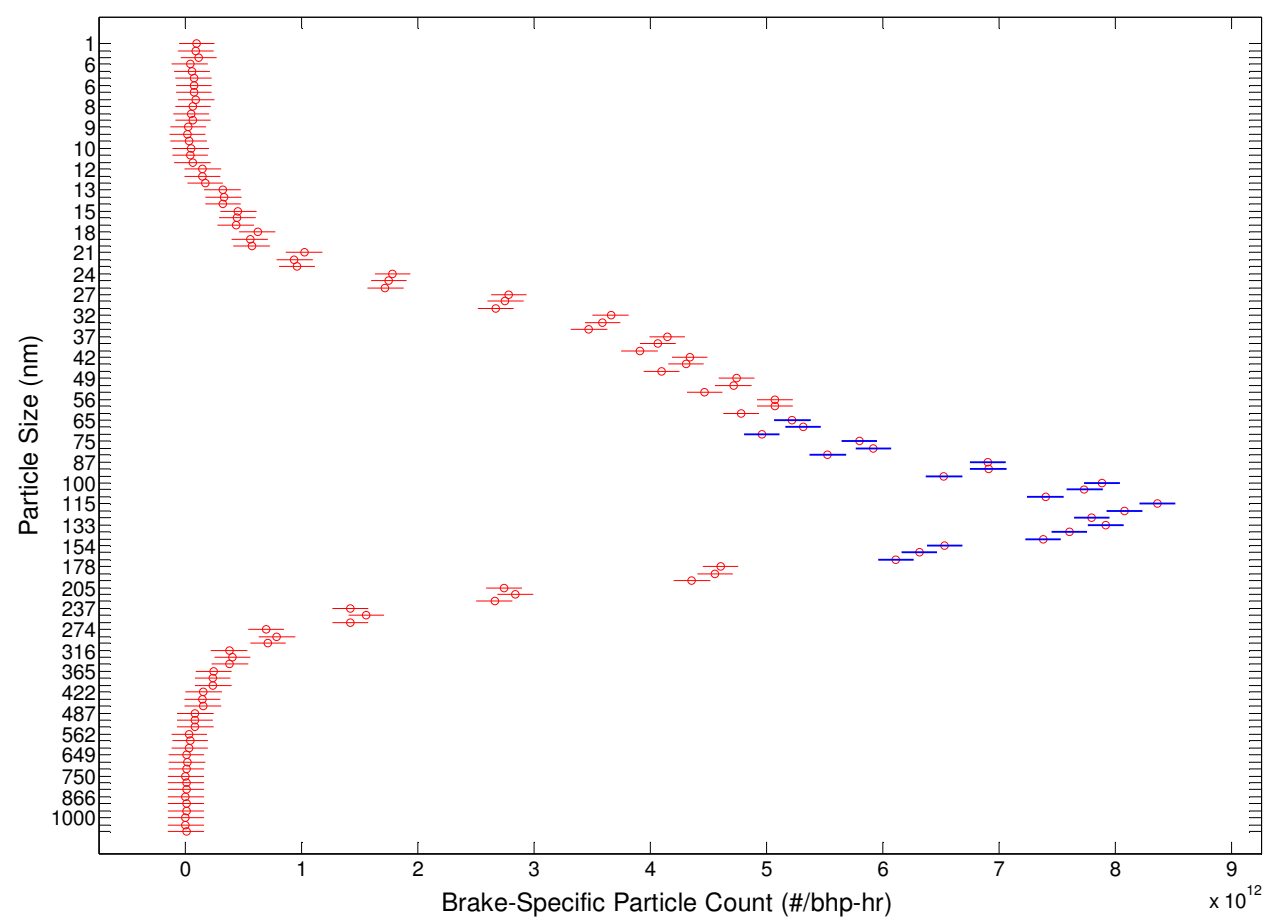

Figure A-63 Particle Sizes that Have Significantly Different Average Values for Mode 8 Due to Humidity Change (According to ANOVA) 


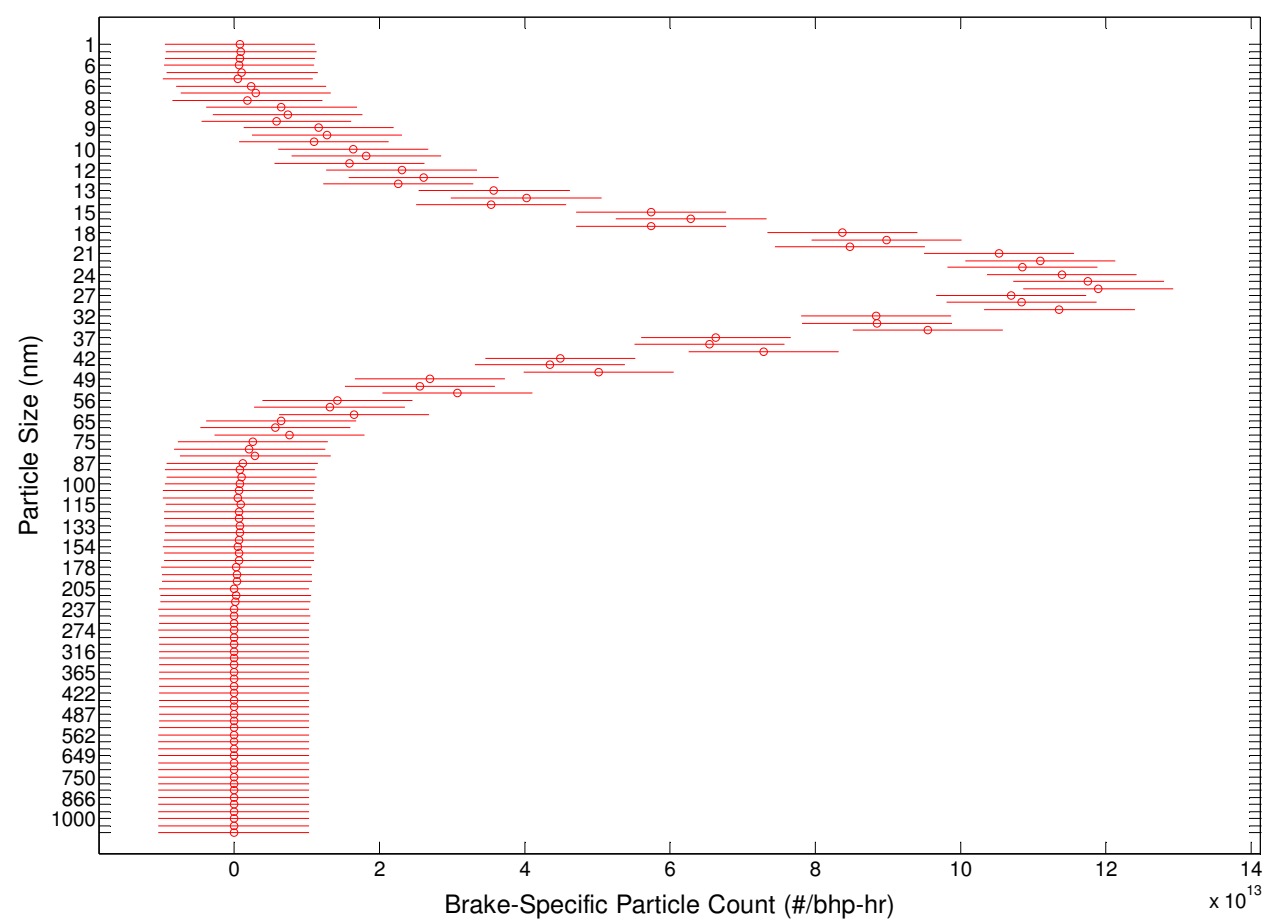

Figure A-64 Particle Sizes that Have Significantly Different Average Values for Mode 9 Due to Humidity Change (According to ANOVA)

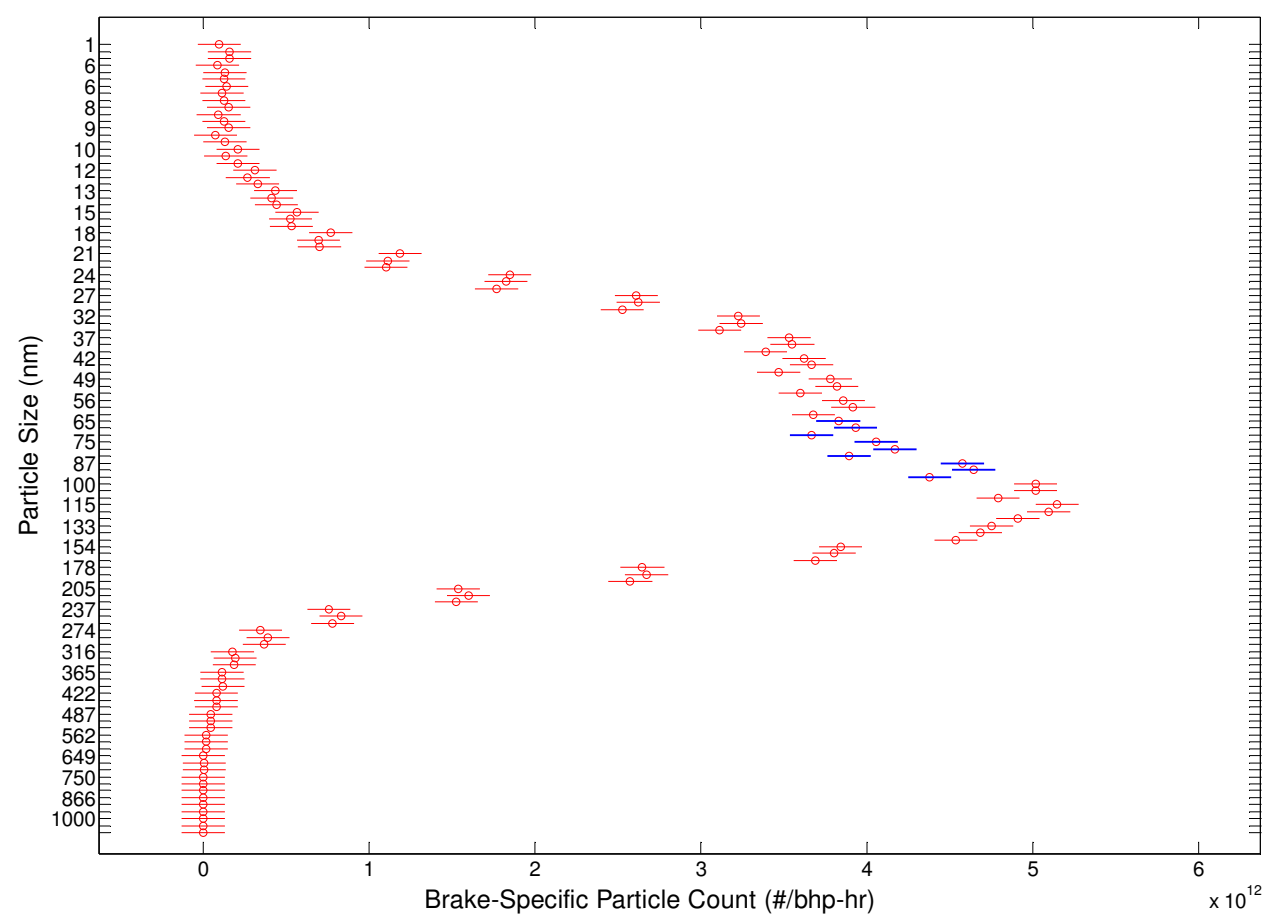

Figure A-65 Particle Sizes that Have Significantly Different Average Values for Mode 10 Due to Humidity Change (According to ANOVA) 


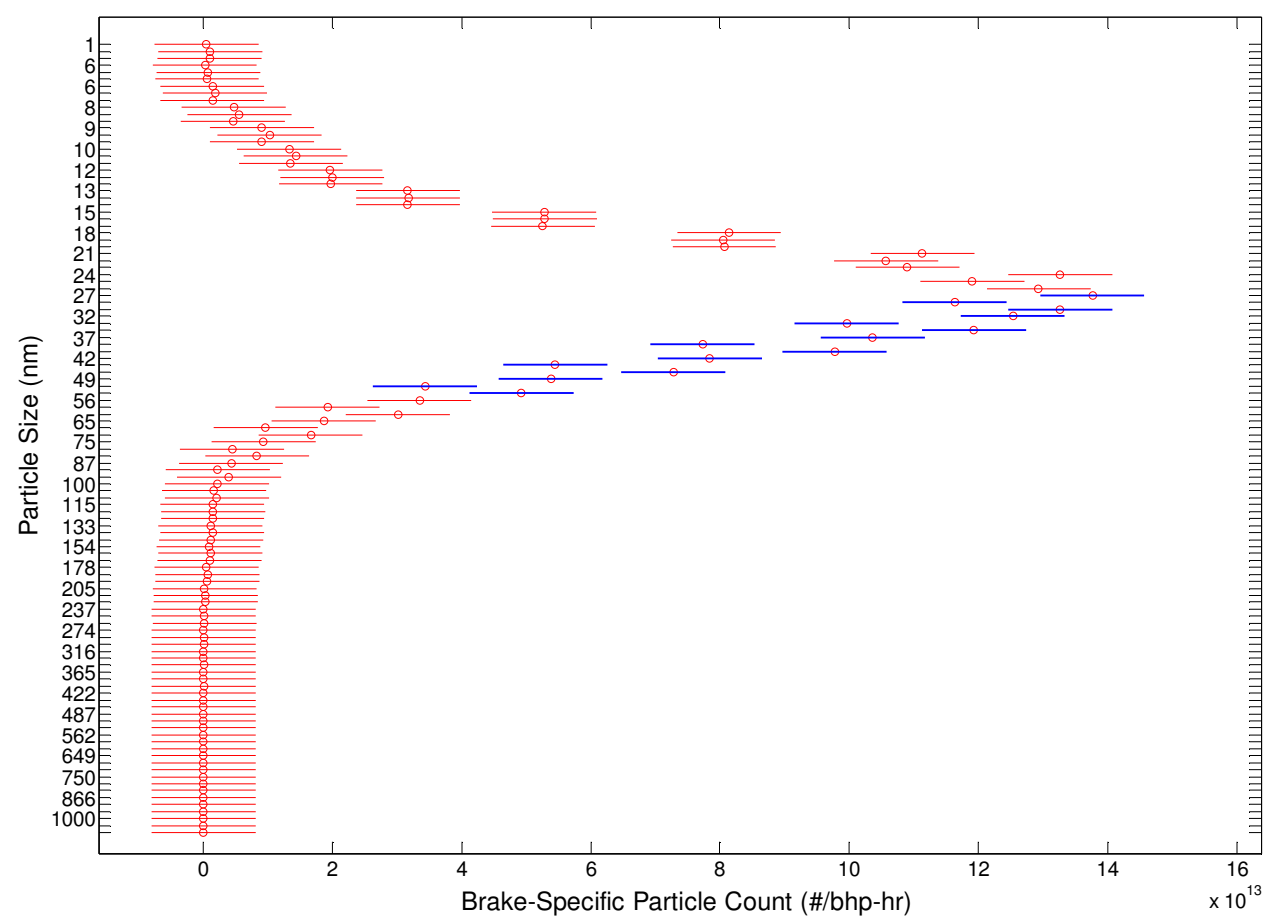

Figure A-66 Particle Sizes that Have Significantly Different Average Values for Mode 11 Due to Humidity Change (According to ANOVA)

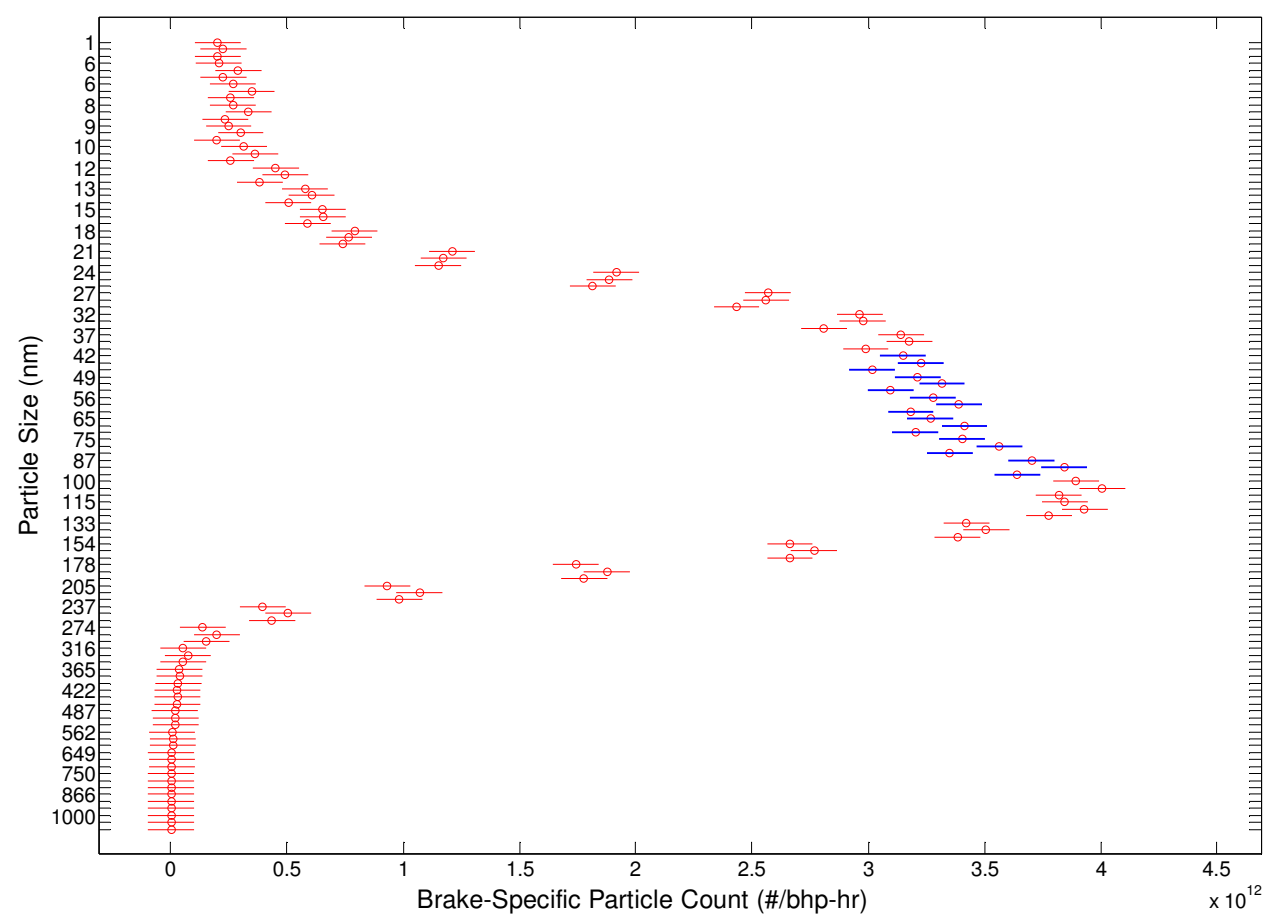

Figure A-67 Particle Sizes that Have Significantly Different Average Values for Mode 12 Due to Humidity Change (According to ANOVA) 


\section{A.7 MATLAB ANOVA CODE}

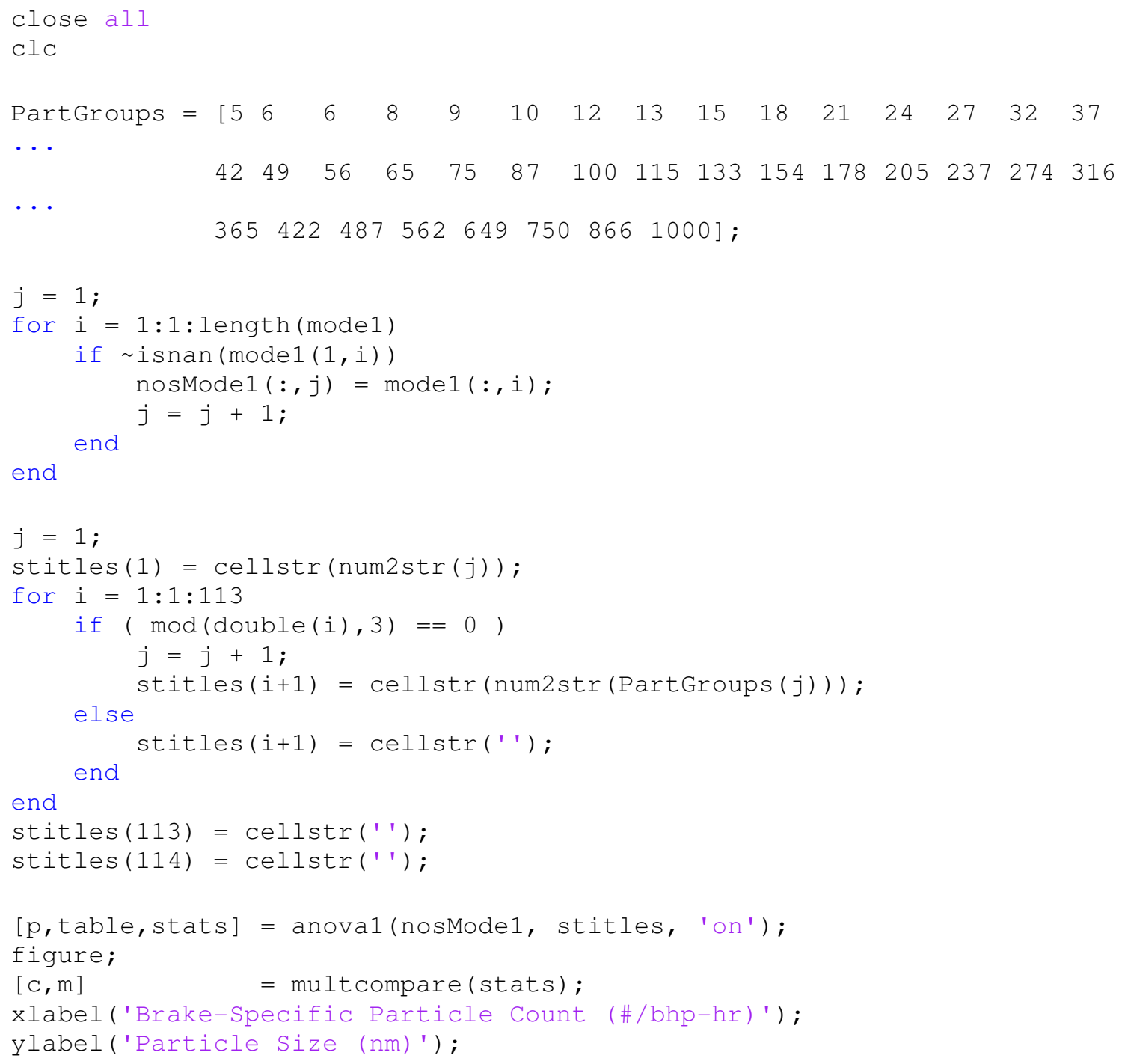




\section{A.8 DILUTION RATIO EFFECTS}

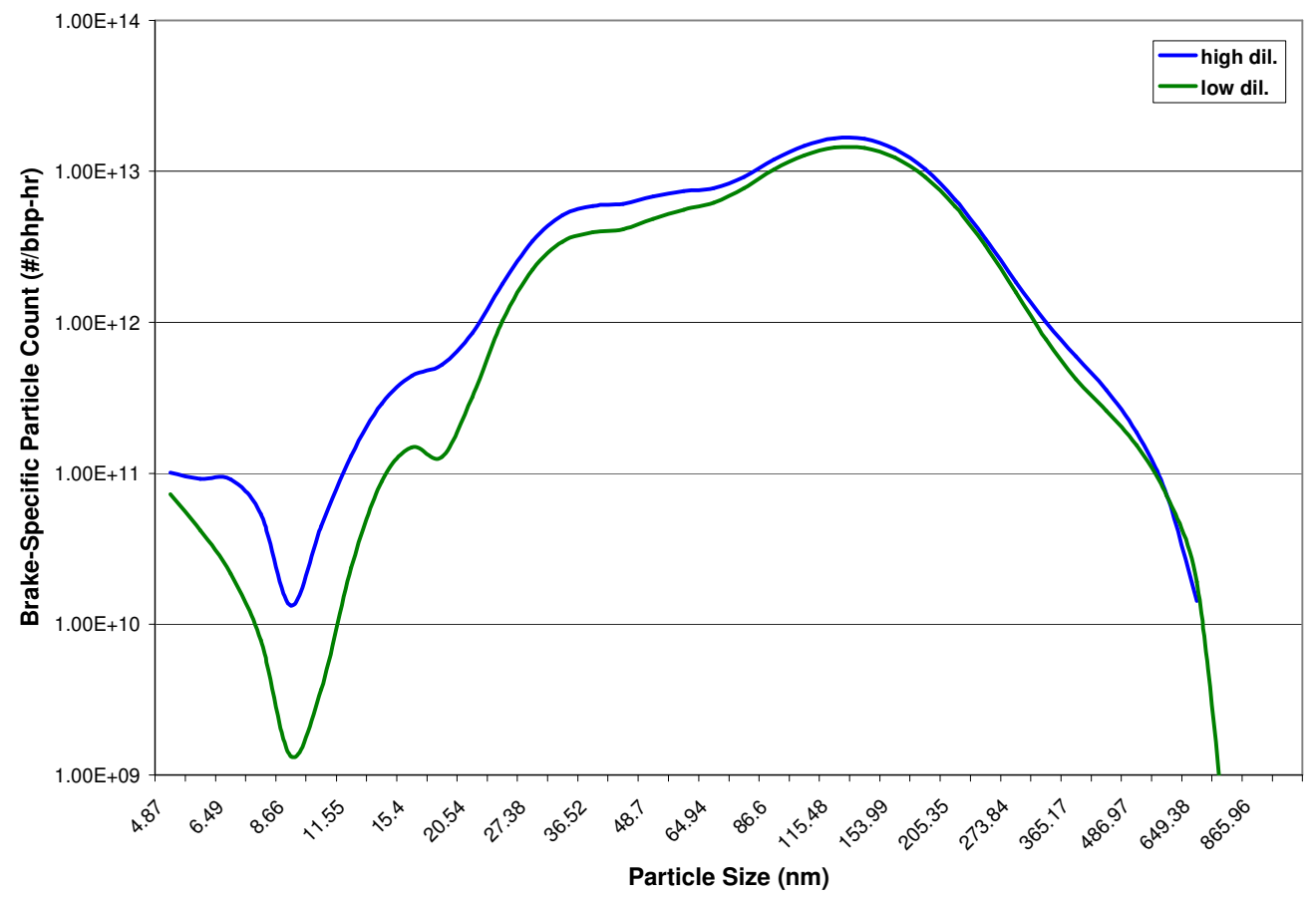

Figure A-68 Particle Concentration Comparison of High and Low Dilution Settings of Mode 2

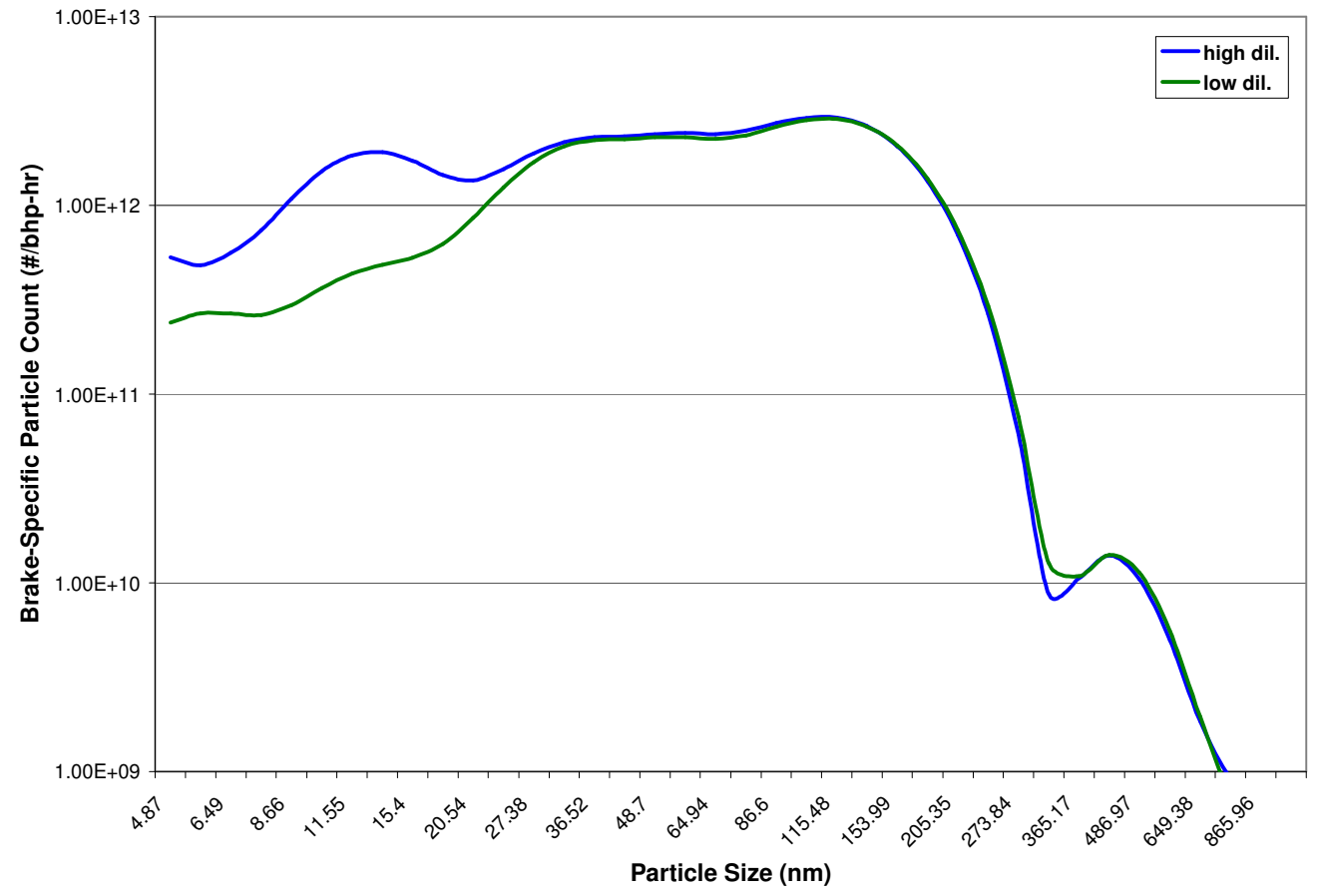

Figure A-69 Particle Concentration Comparison of High and Low Dilution Settings of Mode 5 


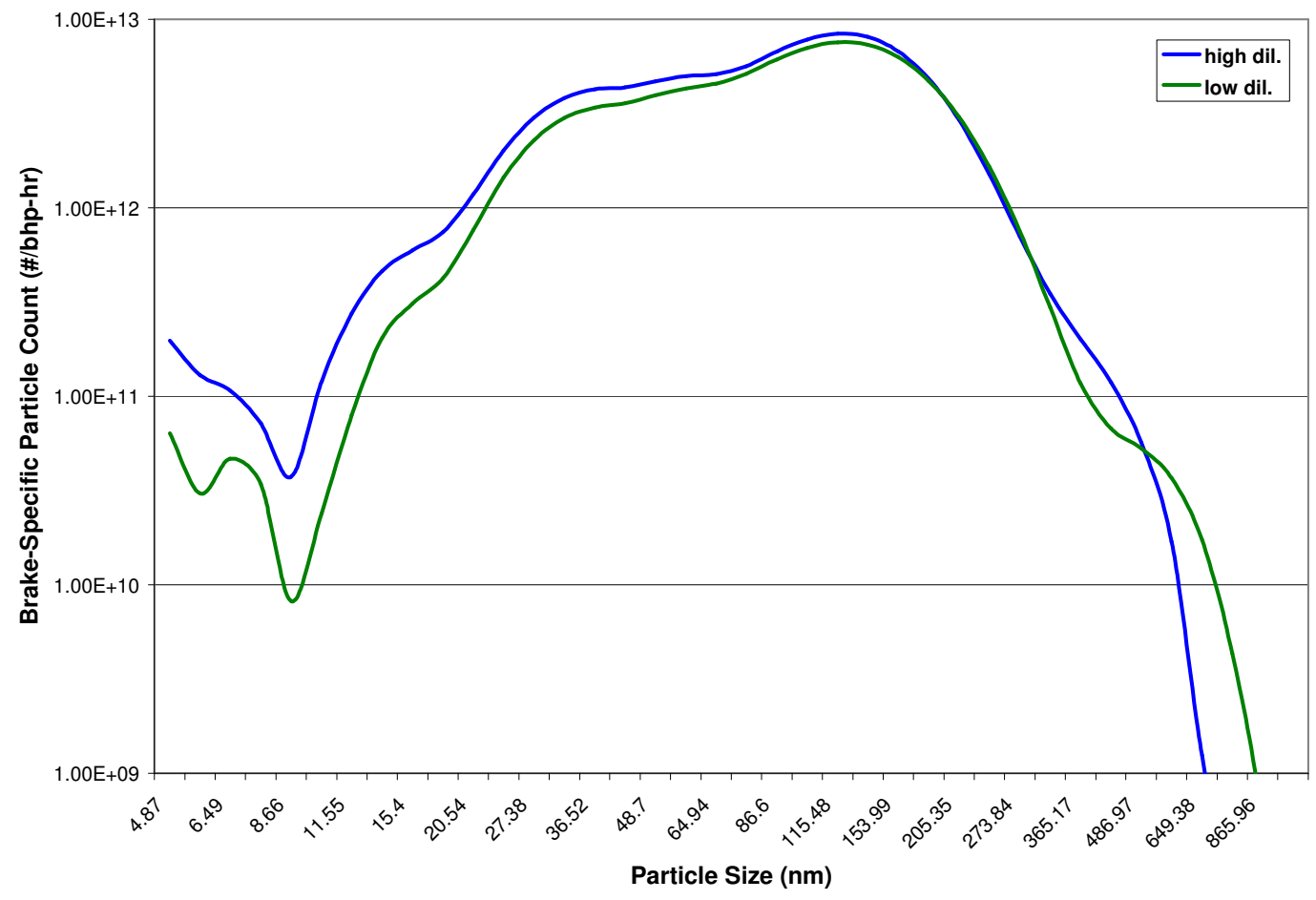

Figure A-70 Particle Concentration Comparison of High and Low Dilution Settings of Mode 6

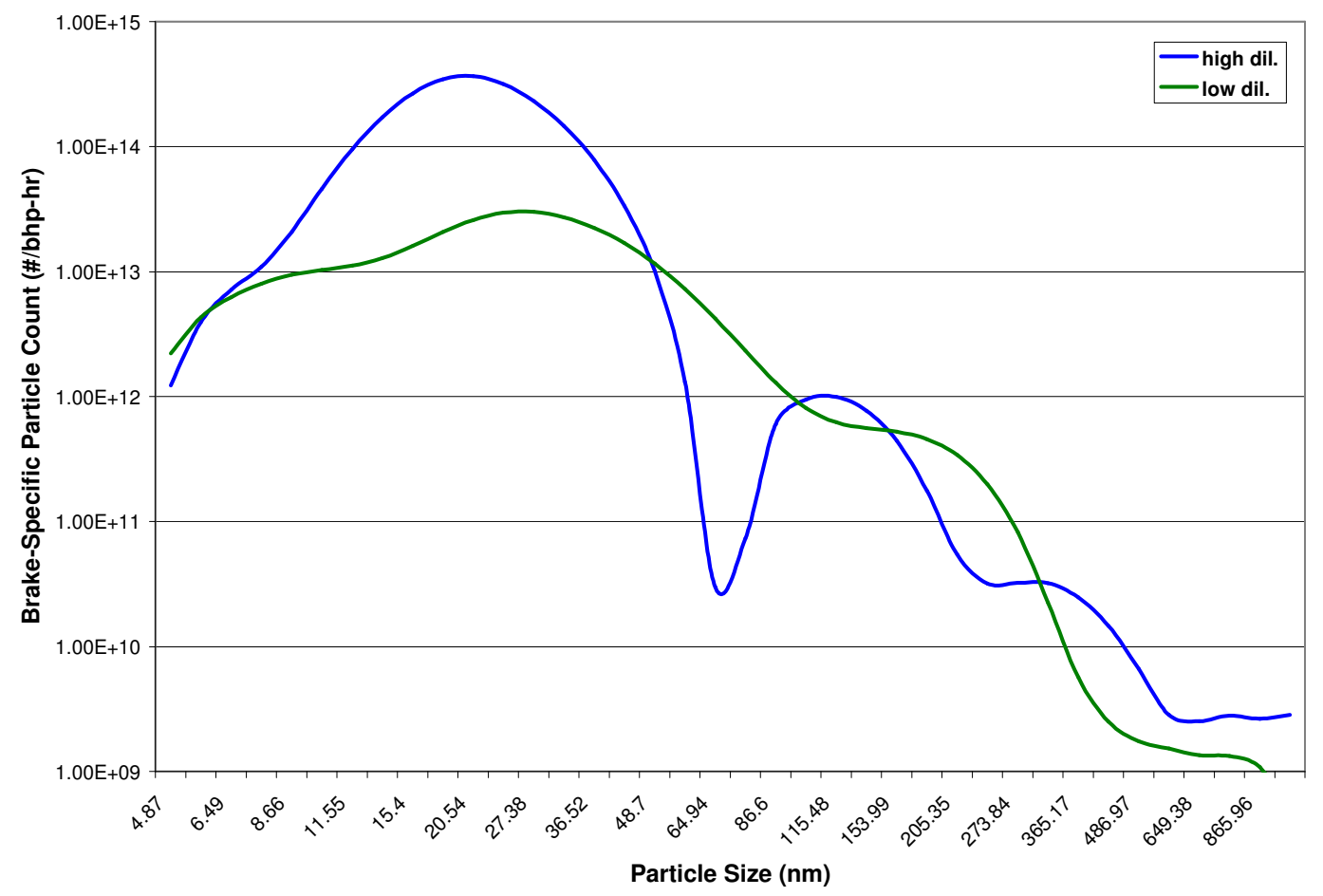

Figure A-71 Particle Concentration Comparison of High and Low Dilution Settings of Mode 7 


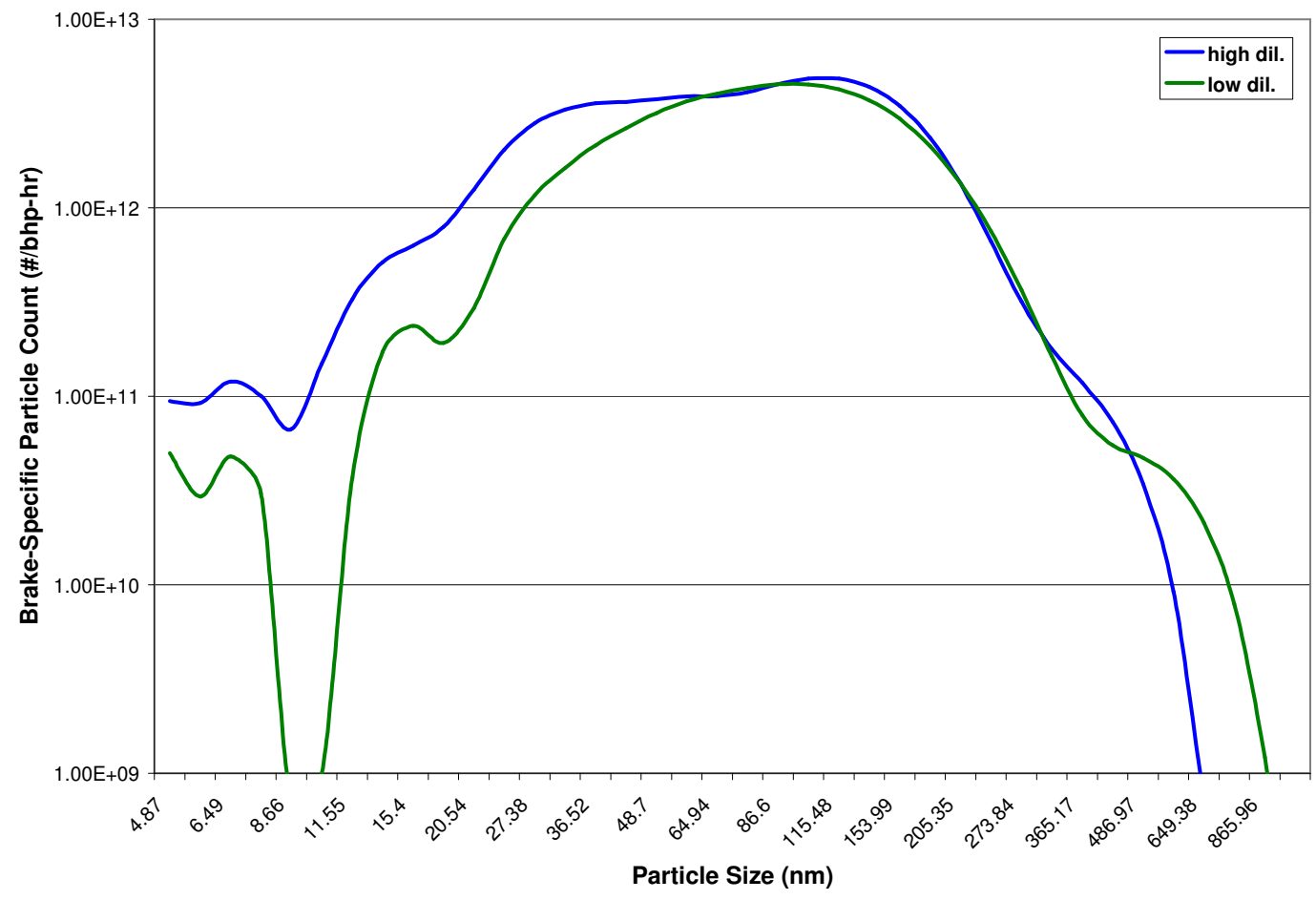

Figure A-72 Particle Concentration Comparison of

High and Low Dilution Settings of Mode 10

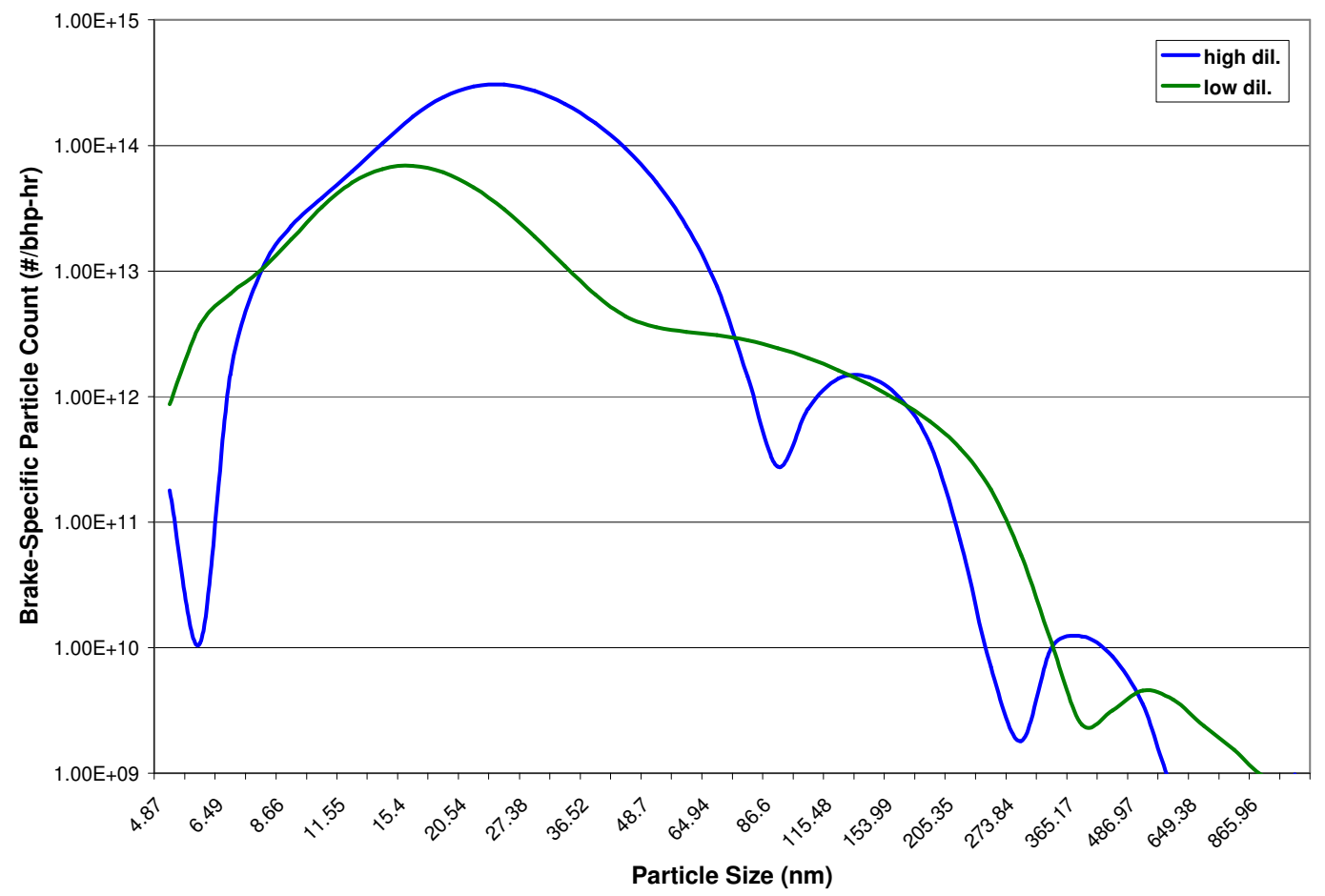

Figure A-73 Particle Concentration Comparison of High and Low Dilution Settings of Mode 11 


\section{A.8.1 High Dilution}
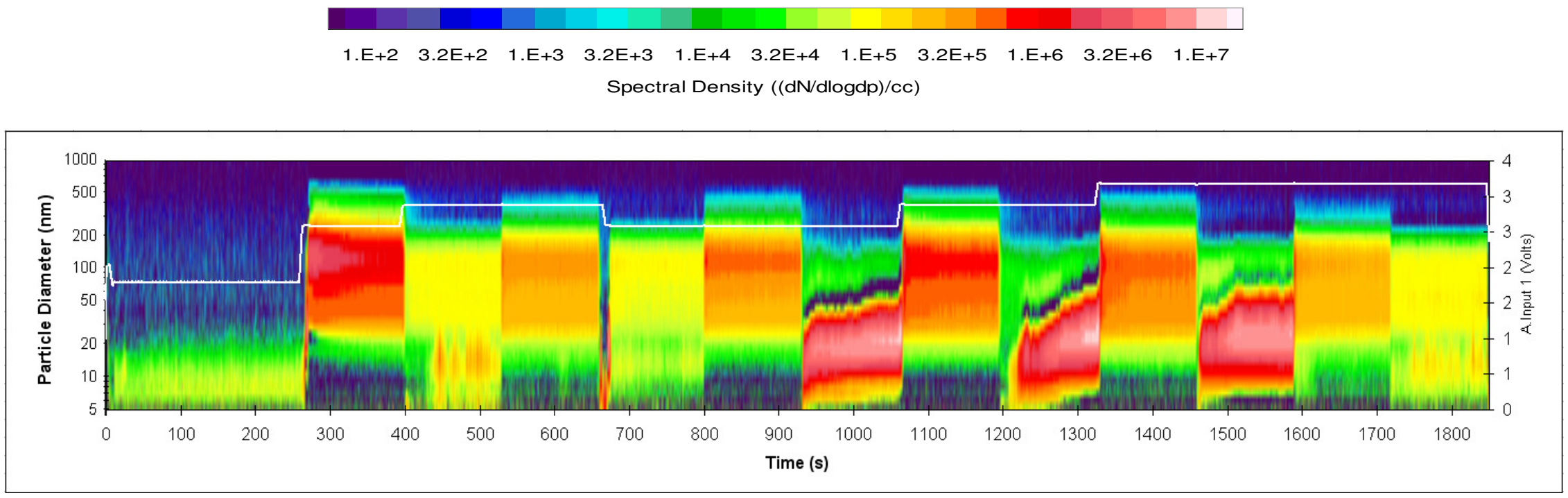

Figure A-74 Continuous Particle Distribution of E02208_01 


\section{A.8.2 Standard Dilution}

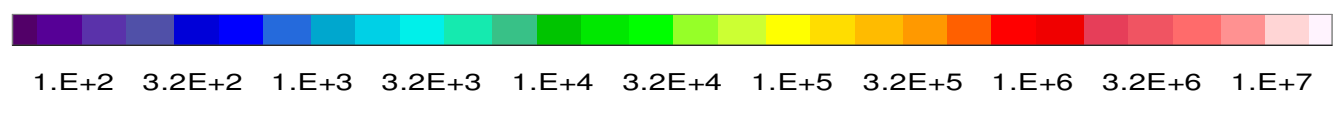

Spectral Density ((dN/dlogdp)/cc)

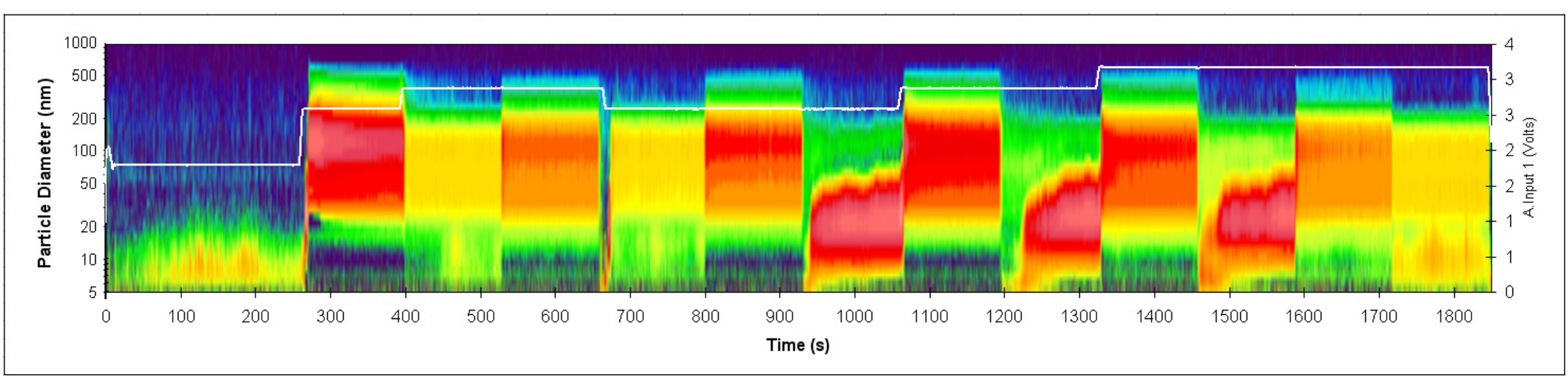

Figure A-75 Continuous Particle Distribution of E02206_02 


\section{A.8.3 Low Dilution}

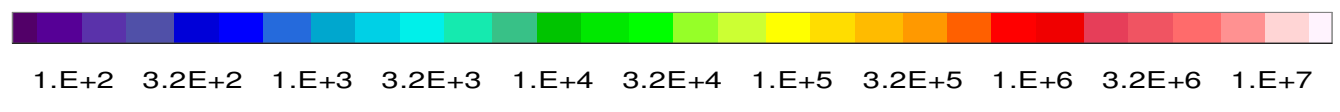

Spectral Density ((dN/dlogdp)/cc)

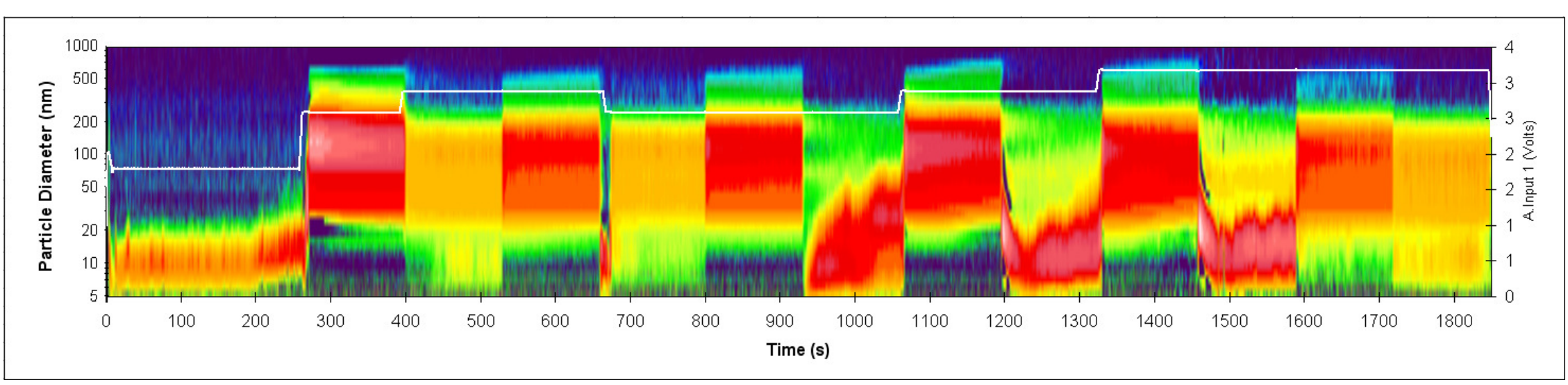

Figure A-76 Continuous Particle Distribution of E02208_02 